\title{
Fragility and excluded minors
}

\author{
Ben Clark
}

\section{VICTORIA UNIVERSITY OF WELLINGTON \\ Te Whare Wānanga o te Ūpoko o te Ika a Māui}

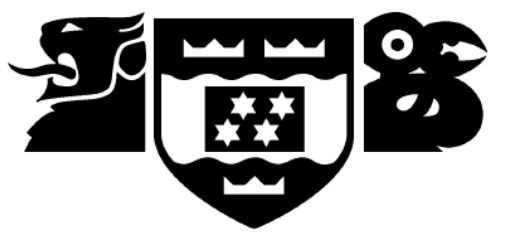

School of Mathematics, Statistics and Operations Research

Te Kura Mātai Tatauranga, Rangahau Pūnaha

\author{
A thesis \\ submitted to the Victoria University of Wellington \\ in fulfilment of the requirements for the degree of \\ Doctor of Philosophy \\ in Mathematics.
}

Victoria University of Wellington

2015 



\begin{abstract}
Let $\mathscr{N}$ be a set of matroids. A matroid, $M$, is $\mathscr{N}$-fragile, if for every element $e$, either $M \backslash e$ or $M / e$ has no minor isomorphic to a member of $\mathscr{N}$. This thesis gives new results in matroid representation theory that elucidate the relationship between $\mathscr{N}$-fragile matroids and excluded minors.

Let $\mathbb{P}$ be a partial field, and let $\mathscr{N}$ be a set of strong stabilizers for $\mathbb{P}$. The first main result of this thesis establishes a relationship between $\mathscr{N}$-fragile matroids and excluded minors for the class of $\mathbb{P}$-representable matroids. We prove that if an excluded minor $M$ for the class of $\mathbb{P}$-representable matroids has a pair of elements $a, b$ such that $M \backslash a, b$ is 3 -connected with an $\mathscr{N}$-minor, then either $M$ is close to an $\mathscr{N}$-minor or $M \backslash a, b$ is $\mathscr{N}$-fragile. The result motivates a study of the structure of $\mathbb{P}$-representable $\mathscr{N}$-fragile matroids.

The matroids $U_{2,5}$ and $U_{3,5}$ are strong stabilizers for the $\mathbb{U}_{2}$ and $\mathbb{H}_{5}$ partial fields. The second main result of this thesis is a structural characterisation of the $\mathbb{U}_{2}$ and $\mathbb{H}_{5}$-representable $\left\{U_{2,5}, U_{3,5}\right\}$-fragile matroids. We prove that these matroids can be constructed from $U_{2,5}$ and $U_{3,5}$ by a sequence of moves, where, up to duality, each move consists of a parallel extension followed by a delta-wye or a generalised delta-wye exchange.

Finally, we obtain a bound on the size of an excluded minor $M$ for the class of $\mathbb{U}_{2^{-}}$ or $\mathbb{H}_{5}$-representable matroids with the property that $M$ has a pair of elements $a, b$ such that $M \backslash a, b$ is 3-connected with a $\left\{U_{2,5}, U_{3,5}\right\}$-minor. Our proof uses the first and second main results of this thesis.
\end{abstract}





\section{Acknowledgements}

Foremost thanks go to my supervisor Geoff Whittle for his support and generosity, and for the many helpful conversations we have shared.

I would like to thank my coauthors, Dillon Mayhew, James Oxley, Charles Semple, and Stefan van Zwam, for their contributions to the research in this thesis and for the opportunity to collaborate with them. I also thank Michael Welsh for helping with Sage and LaTeX.

I have greatly enjoyed my time at Victoria. My thanks go to the staff and graduate students who have created a stimulating environment in which to study matroid theory.

Finally, I would like to thank my family, friends, and Mawsi for the support and encouragement they provide. 



\section{Contents}

1 Introduction 1

$1.1 \quad$ Preliminaries $\ldots \ldots \ldots \ldots \ldots \ldots$

2 Excluded minors are almost fragile 15

$2.1 \quad$ Introduction . . . . . . . . . . . . . . . . . . 15

2.2 Preliminaries . . . . . . . . . . . . . . . . . 18

2.3 The setup . . . . . . . . . . . . . . . . . 24

2.4 Strong elements . . . . . . . . . . . . . . . . . . . . 28

2.5 The gadget case . . . . . . . . . . . . . . 33

2.6 Robust elements . . . . . . . . . . . . . . . . . . . . . 39

2.7 The main results . . . . . . . . . . . . . . . . . . . . . . 46

$3\left\{U_{2,5}, U_{3,5}\right\}$-fragile matroids

3.1 Introduction . . . . . . . . . . . . . . . . . . 62

3.2 Preliminaries . . . . . . . . . . . . . . 68

3.3 The class $\mathscr{P} \ldots \ldots \ldots \ldots \ldots \ldots$

3.4 Path sequence rearrangement $\ldots \ldots \ldots \ldots$

3.5 The setup . . . . . . . . . . . . . . . . 80

3.6 Guts case $\ldots \ldots \ldots \ldots$

3.7 Blocking case . . . . . . . . . . . . . . 85

3.8 Spanning and cospanning case $\ldots \ldots \ldots$. . . . . . . . 90 
$3.9 \quad$ Case analysis $\ldots \ldots \ldots \ldots$. . . . . . . . . . . . . . . . . 94

\begin{tabular}{|lr}
4 On excluded minors & 103
\end{tabular}

4.1 Introduction . . . . . . . . . . . . . . . . . 103

4.2 Preliminaries . . . . . . . . . . . . . . . 105

4.3 Robust basis wins . . . . . . . . . . . . . . . . . . . . . . . 109

4.4 Blocking a triad . . . . . . . . . . . . . . . . 111

4.5 New pairs for deletion and contraction . . . . . . . . . . 115

4.6 The bound . . . . . . . . . . . . . . . . . . . . . . . . 122

\begin{tabular}{ll}
\hline Bibliography & 127
\end{tabular}

\begin{tabular}{ll}
\hline Index & 131
\end{tabular} 


\section{Chapter 1}

\section{Introduction}

Matroids axiomatise the properties of dependence displayed by finite sets of vectors in a vector space. A matroid $M$ that arises from the column vectors of a matrix $A$ with entries in a field $\mathbb{F}$ is said to be $\mathbb{F}$-representable, and the matrix $A$ is called an $\mathbb{F}$-representation of $M$. Of course, for a fixed field $\mathbb{F}$, not all matroids are $\mathbb{F}$-representable. It is a fundamental problem in matroid representation theory to characterise the matroids that are $\mathbb{F}$-representable.

The class of $\mathbb{F}$-representable matroids is closed under the minor operations, so an attractive way to characterise the class of $\mathbb{F}$-representable matroids is via a list of excluded minors, that is, a list of minor-minimal matroids that are not $\mathbb{F}$-representable. In general, the number of excluded minors for a class of $\mathbb{F}$ representable matroids can be infinite, and moreover the list of excluded minors can be as rich as possible [16]. However, in contrast to the situation for infinite fields, Rota conjectured in 1970 that for each finite field $\mathbb{F}$, there are, up to isomorphism, only finitely many excluded minors for the class of $\mathbb{F}$-representable matroids. Rota's Conjecture is probably the most well-known conjecture in matroid theory. Geelen, Gerards, and Whittle [7] recently announced, after 15 years of intensive research, that they have extended the Graph Minor Structure Theorem of Robertson and Seymour [30] to matroids representable over finite fields, and, combining this powerful tool with other results, they have proved Rota's Conjecture [6]. In addition to being a beautiful result, the proof of Rota's Conjecture has a number of important theoretical implications. For example, certifying non-representability over a finite field requires only a constant number of rank evaluations [10]. 
Although Geelen, Gerards, and Whittle have settled Rota's Conjecture, the problem of finding the complete list of excluded minors, or even finding a computable bound on the number of excluded minors, remains an open problem for all but three finite fields: In 1958, Tutte showed that there is exactly one excluded minor for the class of matroids representable over GF(2) (see [20, Theorem 6.5.4]); In 1979, Bixby and Seymour independently proved that there are four excluded minors for GF(3) (see [20, Theorem 10.2.1]); In 2000, Geelen, Gerards, and Kapoor [5] proved that there are seven excluded minors for GF(4). As the timeline of progress suggests, new excluded-minor characterisations are extremely difficult to obtain, and each requires the development of new techniques. This thesis is part of an on-going research project with the aim of extending the techniques and ideas of [5] to find new excluded-minor characterisations. In particular, it is hoped that the project will lead to an excluded-minor characterisation of the class of $\mathrm{GF}(5)$-representable matroids. Given the obstacles that we shall describe next, and the explosion in the number of excluded minors (see [17]), the class of GF(5)representable matroids is almost certainly the last open class of $\mathbb{F}$-representable matroids for which an excluded-minor characterisation is possible.

A serious obstacle to extending the techniques of [5] to $\mathrm{GF}(5)$ and other fields is that, in general, a matroid may have inequivalent representations over a field. Recall that two $\mathbb{F}$-representations of a matroid $M$ are equivalent if one can be obtained from the other by some sequence of elementary row operations, columnscalings, and field automorphisms. The excluded-minor characterisations of the classes of GF(2)-, GF(3)-, and GF(4)-representable matroids all relied crucially on being able to control the number of inequivalent representations over these fields: matroids are uniquely representable over $\mathrm{GF}(2)$ and $\mathrm{GF}(3)$; in general, a matroid can have inequivalent representations over GF(4), but a result of Kahn [14] shows that a viable form of unique representability can be recovered if we impose some connectivity restrictions and we fix a GF(4)-representation of a $U_{2,4^{-}}$ minor. We say that $U_{2,4}$ is a strong stabilizer for $\mathrm{GF}(4)$ (see [8]), which will be defined in generality in Section 1.1 . Kahn conjectured further that imposing 3 -connectivity would be enough to bound the number of inequivalent representations over any finite field, but later Oxley, Vertigan, and Whittle [23] proved that 3-connected matroids can have up to six inequivalent $\mathrm{GF}(5)$-representations, and that no bound exists on the number of inequivalent $\mathrm{GF}(q)$-representations for $q>5$. 
The result of Oxley, Vertigan, and Whittle [23] shows that, in contrast to the situation for $\mathrm{GF}(4)$, we cannot control inequivalent representations over $\mathrm{GF}(5)$ with 3-connectivity. However, the existence of a bound on the number of inequivalent representations for 3-connected $\mathrm{GF}(5)$-representable matroids gives rise to some natural subclasses, namely the 3-connected GF(5)-representable matroids with at least $k$ inequivalent representations for $k=1,2, \ldots, 6$. Pendavingh and Van Zwam [27] proved that, for such matroids with a $\left\{U_{2,5}, U_{3,5}\right\}$-minor, each of these subclasses corresponds to a class of matroids representable over a partial field $\mathbb{H}_{1}, \mathbb{H}_{2}, \ldots, \mathbb{H}_{5}=\mathbb{H}_{6}$, the so-called Hydra- $k$ partial field for $k \in\{1,2, \ldots, 6\}$. Roughly speaking, a partial field is an algebraic structure like a field, and the familiar matroid notions for representation extend from fields to partial fields in the natural way. Partial fields will be formally defined in Section 1.1. It follows from a result of Whittle [37] that we can recover unique representability in the base class of this family, the class of $\mathbb{H}_{5}$-representable matroids, provided we maintain 3 -connectivity and we fix an $\mathbb{H}_{5}$-representation of a $\left\{U_{2,5}, U_{3,5}\right\}$-minor.

We can therefore view the class of $\mathbb{H}_{5}$-representable matroids as an appropriate setting for extending the techniques of [5]. In theory, while also being of interest in its own right, an excluded-minor characterisation of the class of $\mathbb{H}_{5}$-representable matroids gives some traction towards an excluded-minor characterisation of the class of GF(5)-representable matroids. Van Zwam [35] proved that the excluded minors for the class of $\mathbb{H}_{5}$-representable matroids that belong to the class of $\mathbb{H}_{4}$ representable matroids can be used to recover unique representability over the $\mathbb{H}_{4}$ partial field. We would then seek an excluded-minor characterisation of the class of $\mathbb{H}_{4}$-representable matroids, and so on. Thus an excluded-minor characterisation of the class of $\mathbb{H}_{5}$-representable matroids can be viewed as the first major goal of the project.

In addition to studying the class of $\mathbb{H}_{5}$-representable matroids, we can simultaneously study the closely-related subclass of 2-regular or $\mathbb{U}_{2}$-representable matroids. Indeed, Semple [32] proved that we can recover unique representability for the class of $\mathbb{U}_{2}$-representable representable matroids if we impose 3-connectivity and fix a $\mathbb{U}_{2}$-representation of a $\left\{U_{2,5}, U_{3,5}\right\}$-minor. To motivate our interest in this class of matroids, recall that the $\mathbb{U}_{2}$ partial field was introduced by Semple [32] as part of the family of $k$-regular or $\mathbb{U}_{k}$ partial fields for $k \geq 0$. The class of $\mathbb{U}_{0}$-representable matroids is the well-known class of regular matroids, that is, the class of matroids that are $\mathbb{F}$-representable for any field $\mathbb{F}$. The next class 
of $\mathbb{U}_{1}$-representable matroids, also known as the class of near-regular matroids, corresponds to the class of matroids that are $\mathbb{F}$-representable for all fields $\mathbb{F}$ such that $|\mathbb{F}| \geq 3$ (see Whittle [36]). Excluded-minor characterisations have been given for both the class of regular matroids (see [20, Theorem 10.1.1]), and the class of near-regular matroids [12]. Unfortunately, the class of $\mathbb{U}_{2}$-representable matroids is only a proper subclass of the class of matroids that are $\mathbb{F}$-representable for all fields $\mathbb{F}$ such that $|\mathbb{F}| \geq 4$. However, we believe that the excluded minors for the class of $\mathbb{U}_{2}$-representable matroids would help to illuminate the structure of matroids that are $\mathbb{F}$-representable for all fields $\mathbb{F}$ such that $|\mathbb{F}| \geq 4$, and would certainly raise interesting questions for future research.

Having established the classes of matroids of interest, we seek to generalise and, where possible, upgrade the techniques of [5]. A specific goal of the project, and in particular of this thesis, is to exploit the connection between excluded minors and the structure of "fragile" matroids. For a set $\mathscr{N}$ of matroids, we say that a matroid, $M$, is $\mathscr{N}$-fragile, if for every element $e$, either $M \backslash e$ or $M / e$ has no minor isomorphic to a member of $\mathscr{N}$. The connection beween fragility and excluded minors was first used implicitly in [5], and, roughly speaking, we can identify two steps that are important in bounding the size of an excluded minor. One step involves showing that an excluded minor for the class of GF(4)-representable matroids is not far away from a $\left\{U_{2,4}\right\}$-fragile minor, while the other step uses the structure of the $\mathrm{GF}(4)$-representable $\left\{U_{2,4}\right\}$-fragile matroids to bound the size of this $\left\{U_{2,4}\right\}$-fragile minor. It is these two steps that we generalise in this thesis.

We note that evidence to support a general connection between excluded minors and classes of $\mathscr{N}$-fragile matroids can be found already in the work of Mayhew, Whittle, and Van Zwam [19]. Recall that the branch-width of a matroid $M$ is, roughly speaking, an integer $k$ that encodes the property that $M$ can be decomposed in a tree-like way into $k$-separating pieces. They prove that, for any partial field $\mathbb{P}$, if the $\mathscr{N}$-fragile matroids in the class of $\mathbb{P}$-representable matroids have bounded branch-width, where $\mathscr{N}$ is a set of strong stabilizers for $\mathbb{P}$, then there are only finitely many excluded-minors for the class of $\mathbb{P}$-representable matroids that have an $\mathscr{N}$-minor. Thus the work in this thesis can be seen as the natural next step in further exploiting this connection: to see what can be gained by employing exact structure as in [5] over qualitative structure in the form of branch-width.

In Chapter 2, we generalise the property that an excluded minor for the class of $\mathrm{GF}(4)$-representable matroids is not far away from being a GF(4)-representable 
$\left\{U_{2,4}\right\}$-fragile matroid. We prove the following result.

Theorem 1.0.1. Let $\mathbb{P}$ be a partial field, let $M$ be an excluded minor for the class of $\mathbb{P}$-representable matroids, and let $N$ be a strong $\mathbb{P}$-stabilizer minor of $M$ such that $|E(N)| \geq 4$ and $N$ is neither a wheel nor a whirl. If $M$ has a pair of elements $a, b$ such that $M \backslash a, b$ is 3-connected with an $N$-minor, then there is some excluded minor $M^{\prime}$ that is $\Delta$ - $\nabla$-equivalent to $M^{\prime \prime} \in\left\{M, M^{*}\right\}$ with a minor $N^{\prime} \in\left\{N, N^{*}\right\}$, and a pair of elements $a^{\prime}, b^{\prime}$ such that $M^{\prime} \backslash a^{\prime}, b^{\prime}$ is 3-connected with an $N^{\prime}$-minor such that at least one of the following holds:

(a) $\left|E\left(M^{\prime}\right)\right| \leq\left|E\left(N^{\prime}\right)\right|+16$; or

(b) $\min \left\{r\left(M^{\prime}\right), r^{*}\left(M^{\prime}\right)\right\} \leq \max \left\{r\left(N^{\prime}\right), r^{*}\left(N^{\prime}\right)\right\}+7$; or

(c) $M^{\prime} \backslash a^{\prime}, b^{\prime}$ is an $\left\{N^{\prime}\right\}$-fragile matroid.

In fact, we prove a stronger result that both implies Theorem 1.0.1 and gives us more information about the labelling of the elements of the $N$-fragile matroid $M \backslash a, b$ relative to a basis. We note that, at this time, the bounds obtained in Theorem 1.0.1 are too large for us to feasibly search for such excluded minors. However, we believe that it will be possible to sharpen these bounds in future work.

The hypothesis in Theorem 1.0.1 that $M$ has a pair of elements $a, b$ such that $M \backslash a, b$ is 3-connected with an $N$-minor may seem unusual, since we are currently only guaranteed a pair $a, b$ such that $M \backslash a, b$ is 3-connected up to series pairs with an $N$-minor. However, we strongly believe that, up to duality and a single $\Delta$ - $\nabla$ exchange, only small excluded-minors and those with some specific structure will not possess a pair of elements to delete to be 3-connected with an $N$-minor. To explain some of the grounds for this belief, we note that Williams [39] has proved that if $M$ is a 3-connected matroid with at least 13 elements, then, up to duality and a single $\Delta$ - $\nabla$-exchange, either $M$ has a pair of elements $a, b$ such that $M \backslash a, b$ is 3 -connected or else $M$ is a spike. We therefore anticipate that a Splitter Theorem analogue of Williams' result will be obtained in future research.

In Chapter 3, with Theorem 1.0.1 and the classes of $\mathbb{H}_{5^{-}}$and $\mathbb{U}_{2}$-representable matroids in mind, we characterise the structure of the $\left\{U_{2,5}, U_{3,5}\right\}$-fragile matroids that are $\mathbb{H}_{5}$ - and $\mathbb{U}_{2}$-representable. The motivation for such a characterisation comes from [5], where the property that the only 3 -connected $\left\{U_{2,4}\right\}$-fragile 
matroids that are GF(4)-representable are whirls is used implicitly, and the structure of whirls was key in bounding the size of an excluded minor for the class of GF(4)-representable matroids. Similarly, the only 3-connected $\left\{U_{2,4}\right\}$-fragile matroids that are $\mathbb{U}_{1}$-representable are whirls, which was crucial in bounding the size of an excluded minor for the class of $\mathbb{U}_{1}$-representable matroids in [12]. Thus we believe that Theorem 1.0.1 together with the structure of the 3-connected $\left\{U_{2,5}, U_{3,5}\right\}$-fragile matroids will be enough for us to bound the size of an excluded minor for the classs of $\mathbb{H}_{5}$ - and $\mathbb{U}_{2}$-representable matroids.

We note that the study of classes of $\mathscr{N}$-fragile matroids, sometimes under a different name, includes characterisations of the following classes: the $U_{2,4}$-fragile matroids [25]; the binary $\left\{F_{7}, F_{7}^{*}\right\}$-fragile matroids [34, 4]; the binary $\left\{F_{7}, F_{7}^{*}, M^{*}\left(K_{3,3}\right), M^{*}\left(K_{5}\right)\right\}$-fragile matroids [15]; and the graphic $\left\{M\left(K_{3,3}\right), M\left(K_{5}\right)\right\}$-fragile matroids [11]. We characterise the $\mathbb{H}_{5^{-}}$and $\mathbb{U}_{2^{-}}$ representable $\left\{U_{2,5}, U_{3,5}\right\}$-fragile matroids as follows.

Theorem 1.0.2. Let $M^{\prime}$ be a 3-connected strictly $\left\{U_{2,5}, U_{3,5}\right\}$-fragile $\mathbb{H}_{5}-$ or $\mathbb{U}_{2^{-}}$ representable matroid. Then $M^{\prime}$ is isomorphic to a matroid $M$ for which one of the following holds:

(i) $|E(M)| \leq 9$; or

(ii) $M$ or $M^{*}$ can be obtained from either $U_{2,5}$ or $M_{7,1}$ by gluing wheels to triangles; or

(iii) $M$ has a path of 3-separations $\boldsymbol{P}=\left(P_{1}, \ldots, P_{n}\right)$ such that each end step, $P_{1}$ and $P_{n}$, is either a fan or a 4-element segment or cosegment, and, for each internal step $P_{i}$ of $\boldsymbol{P},\left|P_{i}\right| \leq 3$ and $P_{i}$ is either the guts or coguts of the 3-separation $\left(P_{1} \cup \cdots \cup P_{i}, P_{i+1} \cup \cdots \cup P_{n}\right)$ of $M$.

Our proof of Theorem 1.0 .2 (i) and (ii) uses a result of Chun, Chun, Mayhew, and Van Zwam [3] that reduces the verification of these families to a finite casecheck, which was carried out using Sage Matroid in [2]. Our proof of Theorem 1.0.2 (iii) involves proving a stronger result that describes exactly how a family of $\left\{U_{2,5}, U_{3,5}\right\}$-fragile matroids is constructed. The construction of this family consists of a sequence of operations, each of which involves either gluing a wheel to a triangle, or, up to duality, performing a parallel extension followed by a 4-element generalized $\Delta-\nabla$-exchange. Roughly speaking, a generalized $\Delta-\nabla$-exchange replaces a segment by a cosegment analogous to the way the familiar $\Delta-\nabla$-exchange 
replaces a triangle by a triad. The generalized $\Delta-\nabla$-exchange was introduced by Oxley, Semple, and Vertigan [21], and will be discussed in more detail in Section 1.1 .

We note that it can be easily verified from Theorem 1.0.2 that the $\left\{U_{2,5}, U_{3,5}\right\}$ fragile matroids in the classes of $\mathbb{H}_{5}$ and $\mathbb{U}_{2}$-representable matroids have branchwidth 3. Thus it follows from the work of Mayhew, Whittle, and Van Zwam [19] that there are only finitely many excluded-minors for the classes of $\mathbb{H}_{5}$ and $\mathbb{U}_{2^{-}}$ representable matroids with a $\left\{U_{2,5}, U_{3,5}\right\}$-minor. We hope that having the precise structure given by Theorem 1.0 .2 will enable us to obtain a bound on the size of the excluded minors that is much smaller than the bound implied by the branchwidth.

In Chapter 4, we combine Theorem 1.0.1 and Theorem 1.0.2 to prove the following result.

Theorem 1.0.3. There are finitely many robust excluded minors $M$ for the class of matroids representable over $\mathbb{H}_{5}$ or $\mathbb{U}_{2}$ with the property that $M$ has a pair of elements $a, b$ such that $M \backslash a, b$ is 3-connected with a $\left\{U_{2,5}, U_{3,5}\right\}$-minor.

We note that the bounds obtained in the proof of Theorem 1.0 .3 should be viewed as a first step towards an excluded-minor characterisation of the classes of $\mathbb{H}_{5}$ - and $\mathbb{U}_{2}$-representable matroids. A goal for future research is to significantly reduce the size of the bounds obtained in the proof of Theorem 1.0.3, and to upgrade Theorem 1.0.1, to enable us to feasibly search for all of the excluded minors. We believe that such improvements will be possible using the approach and techniques we have developed by employing a more careful analysis.

On the application of these results to other classes, we note that the fragility structural characterisation is certainly going to be the component of our proof strategy that presents the most difficulties. The only other class that appears within reach is the class of dyadic matroids with a $P_{8}$-minor [18]. It is therefore interesting to observe the structure of $M \backslash a, b$ that we can deduce from Theorem 1.0.1 before we have to appeal specifically to Theorem 1.0.2. An interesting problem for future research would be to investigate if there is some viable structural property of fragile matroids, both finer than branch-width but coarser than an exact structural description, that provides an easier certificate that a matroid is not in a class of fragile matroids.

We now discuss matters related to attribution. Section 1.1 will review relevant 
concepts and results on partial fields selected from the literature. Except where noted, the results of Chapter 2, Chapter 3, and Chapter 4 are new. Chapter 2 consists of joint work with James Oxley, Charles Semple, and Geoff Whittle. Chapter 3 consists of joint work with Dillon Mayhew, Geoff Whittle, and Stefan van Zwam.

We have attempted to make the chapters of this thesis self-contained, with the exception that most material that can be found in Oxley [20] has been omitted. We also note that Chapter 2 and Chapter 3 are independent, so it is possible to read Chapter 3 before the more technical Chapter 2 . We assume throughout this thesis that the reader is familiar with the foundations of matroid theory in Oxley [20]. Any undefined matroid terminology or notation used in this thesis will follow that used in [20].

\subsection{Preliminaries}

Partial fields were introduced by Semple and Whittle [31] to systematically study the classes of matroids that arise when we consider representability over sets of fields, for example the ternary matroids (see [36]). Our treatment here will follow the more recent work of Pendavingh and Van Zwam [28, 27].

A partial field is a pair $(R, G)$, where $R$ is a commutative ring with unity, and $G$ is a subgroup of the group of units of $R$ such that $-1 \in G$.

Suppose that $\mathbb{P}=(R, G)$ is a partial field. We sometimes abuse notation and write $p \in \mathbb{P}$ to mean $p \in G \cup\{0\}$. We note that the name "partial field" comes from the fact that the addition is only a partial binary operation. Thus, if $p+q \in \mathbb{P}$, we say that $p+q$ is defined.

Seeking to define matroid representations over a partial field, we first introduce some matrix notation. Recall that, for ordered sets $X$ and $Y$, an $X \times Y$ matrix $A$ over a partial field $\mathbb{P}$ is a function $A: X \times Y \rightarrow \mathbb{P}$. We denote $A(x, y)$ by $A_{x y}$, and we refer to the element $A_{x y}$ of $\mathbb{P}$ as the entry of $A$ whose row is labelled by $x$ and whose column is labelled by $y$. If $X=(1,2, \ldots, k)$ then we say that $A$ is a $k \times Y$ matrix. Note that, for our purposes, the ordering of $X$ and $Y$ is only significant for the sign of determinants. And since the sign is irrelevant to the underlying matroid structure, we will freely permute rows and columns, always along with 
their labels, throughout this thesis.

Let $\mathbb{P}$ be a partial field, and let $A$ be an $X \times Y$ matrix with entries from $\mathbb{P}$. If every subdeterminant of $A$ is contained in $\mathbb{P}$, then $A$ is a $\mathbb{P}$-matrix. If $A$ is a $\mathbb{P}$-matrix, then the rank of $A$ is the largest value $k$ such that $A$ has a non-zero $k \times k$ subdeterminant. We can now show that $\mathbb{P}$-matrices give rise to matroids as follows.

We now fix some some submatrix notation. Let $A$ be an $X \times Y \mathbb{P}$-maxtrix. If $X^{\prime} \subseteq X$ and $Y^{\prime} \subseteq Y$, then we write $A\left[X^{\prime}, Y^{\prime}\right]$ to denote the submatrix of $A$ induced by $X^{\prime}$ and $Y^{\prime}$. If $Z \subseteq X \cup Y$, then we denote by $A[Z]$ the submatrix induced by $X \cap Z$ and $Y \cap Z$, and by $A-Z$ the submatrix induced by $X-Z$ and $Y-Z$.

Theorem 1.1.1. [27 Theorem 2.8] Let $\mathbb{P}$ be a partial field, and let $A$ be an $X \times Y$ $\mathbb{P}$-matrix. Let

$$
\mathscr{B}=\{X\} \cup\{X \triangle Z|| X \cap Z|=| Y \cap Z \mid, \operatorname{det}(A[Z]) \neq 0\}
$$

Then $\mathscr{B}$ is the set of bases of a matroid on $X \cup Y$.

We say that the matroid in Theorem 1.1 .1 is $\mathbb{P}$-representable, and that $A$ is a $\mathbb{P}$ representation of $M$. We write $M=M[I \mid A]$ if $A$ is a $\mathbb{P}$-matrix, and $M$ is the matroid in Theorem 1.1.1. The class of $\mathbb{P}$-representable matroids has a number of useful properties.

Proposition 1.1.2. [31] Proposition 4.2] Let $\mathbb{P}$ be a partial field. The class of $\mathbb{P}$ representable matroids is closed under duality, minors, direct sums, and 2-sums.

We next describe the structure-preserving maps between partial fields. Suppose that $\mathbb{P}_{1}$ and $\mathbb{P}_{2}$ are partial fields. A function $\phi: \mathbb{P}_{1} \rightarrow \mathbb{P}_{2}$ is a partial-field homomorphism if

(i) $\phi(1)=1$;

(ii) for all $p, q \in \mathbb{P}_{1}, \phi(p q)=\phi(p) \phi(q)$; and

(iii) for all $p, q \in \mathbb{P}_{1}$ such that $p+q$ is defined, $\phi(p)+\phi(q)=\phi(p+q)$.

If $A$ is a $\mathbb{P}$-matrix, and $\phi$ is a function with domain $\mathbb{P}$, then we write $\phi(A)$ to denote the matrix obtained by operating on each entry of $A$ with $\phi$. Partial-field homomorphisms have the following important consequence for matroid representability. 
Proposition 1.1.3. [28 Corollary 2.11] Let $\mathbb{P}_{1}, \mathbb{P}_{2}$ be partial fields, and $\phi: \mathbb{P}_{1} \rightarrow$ $\mathbb{P}_{2}$ be a partial-field homomorphism. Let $A$ be a $\mathbb{P}_{1}$-matrix. Then

(i) $\phi(A)$ is a $\mathbb{P}_{2}$-matrix;

(ii) If $A$ is square, then $\operatorname{det}(A)=0$ if and only if $\operatorname{det}(\phi(A))=0$; and

(iii) $M=M[I \mid A]=M[I \mid \phi(A)]$.

We say that a partial field $\mathbb{P}$ is finitary if there is a partial-field homomorphism $\mathbb{P} \rightarrow \mathrm{GF}(q)$ for some finite field $\mathrm{GF}(q)$.

The goal now is to describe the notions of equivalence on $\mathbb{P}$-representations. Analogous to matroids representable over fields, there are three important operations: row and column scaling, pivoting, and applying partial-field automorphisms. We now describe the latter two operations.

Let $A$ be an $X \times Y \mathbb{P}$-matrix, and let $x \in X$ and $y \in Y$ such that $A_{x y} \neq 0$. Then we define $A^{x y}$ to be the $(X \triangle\{x, y\}) \times(Y \triangle\{x, y\})$ matrix given by

$$
\left(A^{x y}\right)_{u v}= \begin{cases}A_{x y}^{-1} & \text { if } u v=y x \\ A_{x y}^{-1} A_{x v} & \text { if } u=y, v \neq x \\ -A_{x y}^{-1} A_{u y} & \text { if } v=x, u \neq y \\ A_{u v}-A_{x y}^{-1} A_{u y} A_{x v} & \text { otherwise. }\end{cases}
$$

We say that $A^{x y}$ is obtained from $A$ by pivoting on $x y$. Note that after pivoting, $x$ labels a column, and $y$ labels a row.

Let $\mathbb{P}_{1}, \mathbb{P}_{2}$ be partial fields and let $\phi: \mathbb{P}_{1} \rightarrow \mathbb{P}_{2}$ be a partial-field homomorphism. Then $\phi$ is a partial-field isomorphism if

1. $\phi$ is a bijection;

2. $\phi(p)+\phi(q) \in \mathbb{P}_{2}$ if and only if $p+q \in \mathbb{P}_{1}$.

A partial-field isomorphism of the form $\phi: \mathbb{P} \rightarrow \mathbb{P}$ is called a partial-field automorphism.

We can now define the following notions of equivalence for matrices with entries in $\mathbb{P}$. Let $A, A^{\prime}$ be matrices with entries in a partial field $\mathbb{P}$. 
1. If $A^{\prime}$ can be obtained from $A$ by repeatedly scaling rows and columns by non-zero elements of $\mathbb{P}$, then we say that $A$ and $A^{\prime}$ are scaling-equivalent.

2. If $A^{\prime}$ can be obtained from $A$ by repeatedly scaling rows or columns by nonzero elements of $\mathbb{P}$, permuting rows, permuting columns, and pivoting, then we say that $A$ and $A^{\prime}$ are geometrically-equivalent.

3. If $\phi\left(A^{\prime}\right)$ is geometrically-equivalent to $A$ for some partial-field automorphism $\phi$, then we say that $A^{\prime}$ and $A$ are algebraically-equivalent.

It is not difficult to check that these notions of equivalence are indeed equivalence relations. When we speak of equivalent or inequivalent matrices without a prefix, we shall mean algebraically-equivalent. We can also see that equivalent matrices represent the same matroid, as follows.

Proposition 1.1.4. [19] Lemma 2.14] If $A, A^{\prime}$ are equivalent $\mathbb{P}$-matrices, then $M[I \mid A]=M\left[I \mid A^{\prime}\right]$.

Let $M$ be a matroid, and $\mathbb{P}$ be a partial field. We say that $M$ is uniquely representable over $\mathbb{P}$ if, whenever $A$ and $A^{\prime}$ are $\mathbb{P}$-matrices such that $M=M[I \mid A]=$ $M\left[I \mid A^{\prime}\right]$, then $A$ and $A^{\prime}$ are equivalent.

We next describe the notion of a stabilizer and a strong stabilizer for matroids representable over a partial field $\mathbb{P}$. These definitions were introduced in [37, 8].

Let $\mathbb{P}$ be a partial field, and let $M$ and $N$ be 3-connected $\mathbb{P}$-representable matroids such that $N$ is a minor of $M$. Suppose the ground set of $N$ is $X^{\prime} \cup Y^{\prime}$, where $X^{\prime}$ is a basis of $N$. Then $N$ is a $\mathbb{P}$-stabilizer for $M$ if, whenever $A_{1}$ and $A_{2}$ are $X \times Y$ $\mathbb{P}$-matrices (where $X^{\prime} \subseteq X$ and $Y^{\prime} \subseteq Y$ ) such that

(i) $M=M\left[I \mid A_{1}\right]=M\left[I \mid A_{2}\right]$;

(ii) $A_{1}\left[X^{\prime}, Y^{\prime}\right]$ is scaling-equivalent to $A_{2}\left[X^{\prime}, Y^{\prime}\right]$; and

(iii) $N=M\left[I \mid A_{1}\left[X^{\prime}, Y^{\prime}\right]\right]=M\left[I \mid A_{2}\left[X^{\prime}, Y^{\prime}\right]\right]$,

then $A_{1}$ is scaling-equivalent to $A_{2}$.

If $N$ stabilizes $M$ over $\mathbb{P}$, and every representation of $N$ extends to a representation of $M$, then we say $N$ strongly stabilizes $M$ over $\mathbb{P}$. We also say that $N$ is a strong $\mathbb{P}$ stabilizer of $M$. These notions extend in the natural way from matroids to classes 
of matroids. In particular, we say that $N$ is a strong $\mathbb{P}$-stabilizer of a class $\mathscr{M}$ of matroids if $N$ is a strong $\mathbb{P}$-stabilizer of $M$ for every 3-connected matroid $M \in \mathscr{M}$ with an $N$-minor.

We now discuss an important operation that we apply throughout this thesis, the generalized $\Delta-\nabla$-exchange of Oxley, Vertigan, and Semple [21]. We can describe this operation geometrically using the generalized parallel connection. Recall that the generalized parallel connection of matroids $M_{1}$ and $M_{2}$ along $A$, denoted by $P_{A}\left(M_{1}, M_{2}\right)$, is the matroid obtained by gluing $M_{1}$ and $M_{2}$ along a common restriction $A$, where $A$ is a modular flat of $M_{1}$ (see [20]). To define the generalized $\Delta$ - $\nabla$-exchange, consider the rank- $k$ projective geometry $P G(k-1, \mathbb{R})$. Fix a basis $B=\left\{b_{1}, \ldots, b_{k}\right\}$ of $P G(k-1, \mathbb{R})$, and choose a line $L$ that is placed freely relative to $B$. If follows from modularity that, for each $i$, the hyperplane spanned by $B-\left\{b_{i}\right\}$ meets $L$, so we let $a_{i}$ be the point of intersection. Let $A=\left\{a_{1}, \ldots, a_{k}\right\}$, and let $\Theta_{k}$ denote the restriction of $P G(k-1, \mathbb{R})$ to the set $A \cup B$. Then, by construction, the matroid $\Theta_{k}$ has a modular $k$-point line $A$. Thus, if $M$ is a matroid with a $k$-point line restriction labelled by $A$, then the generalized parallel connection of $\Theta_{k}$ and $M$ along $A$ is well-defined, and hence so is $P_{A}\left(\Theta_{k}, M\right) \backslash A$. We let $\Delta_{A}(M)$ denote the matroid $P_{A}\left(\Theta_{k}, M\right) \backslash A$, and we say that $\Delta_{A}(M)$ is obtained from $M$ by performing a generalized $\Delta-\nabla$-exchange on $A$. Note that we also require $A$ to be coindependent in $M$, so that $B$ is the dual of a $k$-point line in $\Delta_{A}(M)$. Thus if $M^{*}$ has a $k$-point line restriction, we also have a dual operation $\nabla_{A}(M)$ defined by $\Delta_{A}\left(M^{*}\right)^{*}$. We note that it is natural to relabel the elements $b_{i}$ by $a_{i}$ after performing a generalized $\Delta$ - $\nabla$-exchange, so $\Delta_{A}(M)$ and $M$ are seen as matroids on the same ground set.

The following result shows that excluded minors for the class of $\mathbb{P}$-representable matroids are closed under generalized $\Delta$ - $\nabla$-exchange.

Theorem 1.1.5. [21] Theorem 1.1] Let $\mathbb{P}$ be a partial field, and let $M$ be an excluded minor for the class of $\mathbb{P}$-representable matroids. If $A$ is a coindependent set of $M$, and $M \mid A$ is isomorphic to a rank-2 uniform matroid, then $\Delta_{A}(M)$ is an excluded minor for the class of $\mathbb{P}$-representable matroids.

In fact, it is proved in [21] that if $M$ is $\mathbb{P}$-representable, then the equivalence classes of $\mathbb{P}$-representations for $M$ and $\Delta_{A}(M)$ are in one-to-one correspondence.

We note that we will only need the 3- and 4-element generalized $\Delta$ - $\nabla$-exchange in this thesis, and that the 3 -element generalized $\Delta$ - $\nabla$-exchange is just the $\Delta-\nabla$ - 
exchange. We defer listing further properties of the generalized $\Delta-\nabla$-exchange until Chapter 3 .

We conclude this section by defining the specific partial fields of interest in this thesis, the $\mathbb{H}_{5}$ and $\mathbb{U}_{2}$ partial fields, and stating some of their key properties.

\subsubsection{Hydra-5 partial field}

Pendavingh and Van Zwam [27] define the Hydra-5 partial field as follows.

$$
\begin{gathered}
\mathbb{H}_{5}=(\mathbb{Q}(\alpha, \beta, \gamma),\langle-1, \alpha, \beta, \gamma, \alpha-1, \beta-1, \gamma-1, \alpha-\gamma, \gamma-\alpha \beta, \\
(1-\gamma)-(1-\alpha) \beta\rangle),
\end{gathered}
$$

where $\alpha, \beta, \gamma$ are indeterminates.

However, rather than thinking of $\mathbb{H}_{5}$-representations using the definition of $\mathbb{H}_{5}$, the reader can intuitively think of $\mathbb{H}_{5}$-representations as matrices with entries in the product ring $\bigotimes_{i=1}^{6} \mathrm{GF}(5)$ whose elements are 6-tuples of $\mathrm{GF}(5)$-elements, with componentwise addition and multiplication, such that the projections onto a single coordinate are pairwise inequivalent GF(5)-representations. Computation for the class of $\mathbb{H}_{5}$-representable matroids is performed using this description (see [2]).

We note that, up to permuting the coordinates, $U_{2,5}$ has a unique representation over $\bigotimes_{i=1}^{6} \mathrm{GF}(5)$ such that the projections onto a single coordinate are pairwise inequivalent GF(5)-representations (see [35, Lemma 7.3.16]). Moreover, Whittle [37] showed that $U_{2,5}$ is a stabilizer for the class of $\mathrm{GF}(5)$-representable matroids. That is, if $M$ is a 3-connected, $\mathrm{GF}(5)$-representable matroid with a $U_{2,5}$-minor, then the $\mathrm{GF}(5)$-representation of $U_{2,5}$ uniquely determines the representation of $M$. Thus we have the following result.

Proposition 1.1.6. $U_{2,5}$ is a strong stabilizer for the class of $\mathbb{H}_{5}$-representable matroids.

Our interest in this partial field is primarily based on the following result, which shows that the class of $\mathbb{H}_{5}$-representable matroids is an important subclass of the class of GF(5)-representable matroids.

Theorem 1.1.7. [27. Lemma 5.17] Let M be a 3-connected matroid. 
1. If $M$ has at least 5 inequivalent representations over $\mathrm{GF}(5)$, then $M$ is representable over $\mathbb{H}_{5}$;

2. If $M$ has a $\left\{U_{2,5}, U_{3,5}\right\}$-minor and $M$ is representable over $\mathbb{H}_{5}$, then $M$ has at least 6 inequivalent representations over $\mathrm{GF}(5)$.

For more details, we refer to [27] and [26].

\subsubsection{2-regular partial field}

The $k$-regular (or $k$-uniform) partial field was defined by Semple [32] as follows. $\mathbb{U}_{k}=\left(\mathbb{Q}\left(\alpha_{1}, \ldots, \alpha_{k}\right),\left\langle U_{k}\right\rangle\right)$, where

$$
U_{k}=\left\{x-y \mid x, y \in\left\{0,1, \alpha_{1}, \ldots, \alpha_{k}\right\}, x \neq y\right\}
$$

and $\alpha_{1}, \ldots, \alpha_{k}$ are indeterminates. We note that the 0-regular partial field is also known as the regular partial field, and the 1-regular partial field is also known as the near-negular partial field.

The next result shows that the class of $\mathbb{H}_{5}$-representable matroids contains the class of $\mathbb{U}_{2}$-representable matroids. Indeed, it shows that $\mathbb{U}_{2}$ is the "most general" partial field over which $U_{2,5}$ is representable (see [35]).

Proposition 1.1.8. [32. Corollary 3.1.2] Let $\mathbb{P}$ be a partial field. The matroid $U_{2, k+3}$ is $\mathbb{P}$-representable if and only if the class of $\mathbb{P}$-representable matroids contains the class of $\mathbb{U}_{k}$-representable matroids.

Semple also proved that $U_{2,5}$ was a uniquely representable stabilizer for the class of $\mathbb{U}_{2}$-representable matroids. Hence we have the following.

Lemma 1.1.9. [32] Lemma 4.2.1, Lemma 6.4.16] $U_{2,5}$ is a strong stabilizer for the class of $\mathbb{U}_{2}$-representable matroids.

For more details, we refer the reader to [32]. 


\section{Chapter 2}

\section{Excluded minors are almost fragile}

Let $M$ be an excluded minor for the class of $\mathbb{P}$-representable matroids, and let $N$ be a 3-connected strong $\mathbb{P}$-stabilizer such that $N$ is neither a wheel nor a whirl. We prove that if $M$ has a pair of elements $a, b$ such that $M \backslash a, b$ is 3-connected with an $N$-minor, then the size of $M$ is bounded or $M \backslash a, b$ has at most 3 elements that are $N$-flexible. Moreover, we prove that, up to replacing $M$ by a $\Delta$ - $\nabla$-equivalent excluded-minor, if $M$ has a pair of elements $a, b$ such that $M \backslash a, b$ is 3-connected with an $N$-minor, then the size of $M$ is bounded or we can choose $a, b$ such that $M \backslash a, b$ is $N$-fragile.

\subsection{Introduction}

Let $M$ and $N$ be matroids, and let $x$ be an element of $M$. If $M \backslash x$ has an $N$-minor, then $x$ is $N$-deletable. If $M / x$ has an $N$-minor, then $x$ is $N$-contractible. If neither $M \backslash x$ nor $M / x$ has an $N$-minor, then $x$ is $N$-essential. If $x$ is both $N$-deletable and $N$-contractible, then we say that $x$ is $N$-flexible. We say that the matroid $M$ is $N$ fragile if no element of $M$ is $N$-flexible. If $M$ is $N$-fragile and has an $N$-minor, then we say that $M$ is strictly $N$-fragile. In this chapter, $N$-fragile will always mean strictly $N$-fragile.

The excluded-minor characterisations for the classes of GF(4)- and $\mathbb{U}_{1}$ representable matroids both utilised the fact that $U_{2,4}$ is a strong stabilizer. Roughly speaking, a matroid $N$ is a strong stabilizer for a partial field $\mathbb{P}$ if, for every $\mathbb{P}$-representable matroid $M$ with an $N$-minor, we are guaranteed that a $\mathbb{P}$ - 
representation of $N$ extends uniquely to a $\mathbb{P}$-represention of $M$. Moreover, as a crucial step towards bounding the size of an excluded minor, these excludedminor characterisations exploit the property that an excluded minor is "close" to a $U_{2,4}$-fragile minor. In this chapter, we generalise this idea by showing that a "large" excluded-minor $M$ for the class of $\mathbb{P}$-representable matroids with a strong $\mathbb{P}$-stabilizer $N$ as a minor is "close" to an $N$-fragile matroid.

We use the Splitter Theorem in our proof, so in this chapter a strong stabilizer $N$ must satisfy certain extra conditions. We require that $N$ be 3 -connected with at least 4 elements, and that $N$ is neither a wheel nor a whirl. Thus, since $U_{2,4}$ is a whirl, we are unable to apply our results as a step towards a new proof of Rota's Conjecture for GF(4). However, we believe that our result will still be useful for finding new excluded-minor characterisations. In particular for the classes of $\mathbb{H}_{5^{-}}$and $\mathbb{U}_{2}$-representable matroids, and for the class of dyadic matroids with a $P_{8}$-minor.

Anticipating future work, we assume that an excluded minor $M$ for the class of $\mathbb{P}$ representable matroids has a pair of elements $a, b$ such that $M \backslash a, b$ is 3-connected with an $N$-minor. For such an excluded minor $M$, we can construct a "companion matrix." That is, a matrix $A$ with entries in $\mathbb{P}$ that is unique up to scaling such that $M \backslash a=M[I \mid(A-a)]$ and $M \backslash b=M[I \mid(A-b)]$. Moreover, we can choose a basis $B$ and cobasis $B^{*}=E(M)-B$ for $M$ such that the $B \times B^{*}$ companion matrix $A$ displays a $2 \times 2$ "bad submatrix". That is, for some $x, y \in B$ the set $\{a, b, x, y\}$ incriminates $(M, A)$. Thus when we say that $B$ is a basis of $M$ in this chapter, we mean a basis such that $B$ gives a $B \times B^{*}$ companion matrix $A$ and for some elements $x, y \in B$ the set $\{a, b, x, y\}$ incriminates $(M, A)$.

Let $M$ be a 3-connected matroid, let $B$ be a basis of $M$, and let $N$ be a 3-connected minor of $M$. An element $e$ of $M$ is $(N, B)$-robust if either

(i) $e \in B$ and $M / e$ has an $N$-minor; or

(ii) $e \in E-B$ and $M \backslash e$ has an $N$-minor.

Note that an $N$-flexible element of $M$ is clearly $(N, B)$-robust for any basis $B$ of $M$. An element $e$ of $M$ is $(N, B)$-strong if either

(i) $e \in B$, and $\operatorname{si}(M / e)$ is 3-connected and has an $N$-minor; or 
(ii) $e \in E-B$, and $\operatorname{co}(M \backslash e)$ is 3-connected and has an $N$-minor.

We will prove that either $M \backslash a, b$ has some local structure containing $\{x, y\}$ that implies a bound on $M$, or that $M$ has a basis $B$ with at most one $(N, B)$-strong element outside of $\{x, y\}$. In the case that some basis $B$ has an $(N, B)$-strong element $z$ outside of $\{x, y\}$, then we will show that $B$ can be chosen such that $\{x, y, z\}$ is a triad of $M \backslash a, b$. We say that a basis $B$ is a robust basis for $M$ if, for any basis $B^{\prime}$ of $M$, the number of $\left(N, B^{\prime}\right)$-robust elements of $M \backslash a, b$ outside of $\left\{x^{\prime}, y^{\prime}\right\}$ is at most the number of $(N, B)$-robust elements of $M \backslash a, b$ outside of $\{x, y\}$. When $M$ has some basis $B$ and an $(N, B)$-strong element outside of $\{x, y\}$, the maximum number of $(N, B)$-robust elements outside of $\{x, y\}$ in a robust basis is subject to the constraint that $B$ displays a triad of the form $\{x, y, z\}$, where $z$ is an $(N, B)$-strong element of $M \backslash a, b$.

The following theorem is the first main result of this chapter. The theorem gives the structure of $M \backslash a, b$ for any pair of elements $a, b$ such that $M \backslash a, b$ is 3-connected with an $N$-minor.

Theorem 2.1.1. Let $M$ be an excluded minor for the class of $\mathbb{P}$-representable matroids, and let $N$ be a strong $\mathbb{P}$-stabilizer. If $M$ has a pair of elements $a, b$ such that $M \backslash a, b$ is 3-connected with an $N$-minor, then at least one of the following holds:

(a) $|E(M)| \leq|E(N)|+16$ or $r(M) \leq r(N)+5$; or

(b) there is some robust basis B for $M$ such that either:

(i) $M \backslash a, b$ is $N$-fragile, and $M \backslash a, b$ has at most one $(N, B)$-robust element $z \in B^{*}-\{a, b\}$ outside of $\{x, y\}$. Moreover, if $z \in B^{*}-\{a, b\}$ is $(N, B)$ robust, then $z$ is an $(N, B)$-strong element of $M \backslash a, b$, and $\{x, y, z\}$ is a triad of $M \backslash a, b$; or

(ii) $M \backslash a, b$ is not $N$-fragile, but the only $N$-flexible elements of $M \backslash a, b$ are contained in a triad $\{x, y, z\}$ of $M \backslash a, b$ for some $(N, B)$-strong $z \in B^{*}$. Moreover, the only $(N, B)$-robust elements of $M \backslash a, b$ are in $\{x, y, z\}$, and $M$ has a 4-element cocircuit $\{p, x, y, z\}$ or 5-element cocircuit $\{a, b, x, y, z\}$ that contains a triangle $\{p, x, y\}$ for some $p \in\{a, b\}$.

A matroid $M^{\prime}$ is $\Delta$ - $\nabla$-equivalent to $M$ if there is some sequence of matroids $M_{1}, \ldots, M_{n}$ such that $M=M_{1}, M^{\prime}=M_{n}$, and, for each $i \in\{2, \ldots, n\}$, there is some 
segment or cosegment $A$ of $M_{i-1}$ such that $M_{i}=\nabla_{A}\left(M_{i-1}\right)$ or $M_{i}=\Delta_{A}\left(M_{i-1}\right)$. Oxley, Semple, and Vertigan [21, Theorem 1.1] proved that excluded minors for the class of $\mathbb{P}$-representable matroids are closed under $\Delta$ - $\nabla$-equivalence.

We can now state the second main theorem of the chapter. The theorem shows that we can eliminate the "not $N$-fragile" case of Theorem 2.1.1(b)(ii), up to $\Delta$ - $\nabla$ equivalence, by choosing a better deletion pair.

Theorem 2.1.2. Let $M$ be an excluded minor for the class of $\mathbb{P}$-representable matroids, and let $N$ be a strong $\mathbb{P}$-stabilizer. If $M$ has a pair of elements $a, b$ such that $M \backslash a, b$ is 3-connected with an $N$-minor, then there is some excluded minor $M^{\prime}$ that is $\Delta$-D-equivalent to $M^{\prime \prime} \in\left\{M, M^{*}\right\}$ with a minor $N^{\prime} \in\left\{N, N^{*}\right\}$, a pair of elements $a^{\prime}, b^{\prime}$ such that $M^{\prime} \backslash a^{\prime}, b^{\prime}$ is 3-connected with an $N^{\prime}$-minor such that at least one of the following holds:

(a) $\left|E\left(M^{\prime}\right)\right| \leq\left|E\left(N^{\prime}\right)\right|+16$; or

(b) $\min \left\{r\left(M^{\prime}\right), r^{*}\left(M^{\prime}\right)\right\} \leq \max \left\{r\left(N^{\prime}\right), r^{*}\left(N^{\prime}\right)\right\}+7$; or

(c) there is some robust basis $B^{\prime}$ for $M^{\prime}$ such that $M^{\prime} \backslash a^{\prime}, b^{\prime}$ is $N^{\prime}$-fragile, and $M^{\prime} \backslash a^{\prime}, b^{\prime}$ has at most one $\left(N^{\prime}, B^{\prime}\right)$-robust element $z \in\left(B^{\prime}\right)^{*}-\left\{a^{\prime}, b^{\prime}\right\}$ outside of $\left\{x^{\prime}, y^{\prime}\right\}$. Moreover, if $z \in\left(B^{\prime}\right)^{*}-\left\{a^{\prime}, b^{\prime}\right\}$ is an $\left(N^{\prime}, B^{\prime}\right)$-robust element, then $z$ is an $\left(N^{\prime}, B^{\prime}\right)$-strong element of $M^{\prime} \backslash a^{\prime}, b^{\prime}$, and $\left\{x^{\prime}, y^{\prime}, z\right\}$ is a triad of $M^{\prime} \backslash a^{\prime}, b^{\prime}$.

This chapter is structured as follows. The next section contains a number of connectivity preliminaries. Section 2.3 contains more preliminaries needed for our setup. In Section 2.4 we estabilish a link between strong elements and a structure called a gadget. In Section 2.5 we bound $M$ in the case when $M \backslash a, b$ has a gadget. In Section 2.6 we show that elements of $M \backslash a, b$ that are robust but not strong give rise to a path of 3-separations of $M \backslash a, b$. Finally, in Section 2.7, we use the structure given by the path of 3 -separations in Section 2.6 to prove the main Theorems.

\subsection{Preliminaries}

In this chapter, when we say that $M^{\prime}$ is a 3-connected matroid, we will assume that $\left|E\left(M^{\prime}\right)\right| \geq 7$ to avoid unnecessary degeneracies. The 3-connected matroid we 
have in mind is $M \backslash a, b$ for an excluded minor $M$, and an excluded minor $M$ with $|E(M)| \leq 8$ is clearly not a problem. When we say that $N$ is a 3 -connected strong $\mathbb{P}$-stabilizer, we assume that a strong $\mathbb{P}$-stabilizer $N$ is a 3 -connected matroid with at least 4 elements, and that $N$ is neither a wheel nor a whirl. The reason for these conditions on $N$ is so we can use the Splitter Theorem. In this section, we gather some necessary connectivity preliminaries.

We use the following result known as Bixby's Lemma.

Lemma 2.2.1. [20] Lemma 8.7.3] Let $M$ be a 3-connected matroid, and let e $\in$ $E(M)$. Then $\operatorname{si}(M / e)$ or $\operatorname{co}(M \backslash e)$ is 3-connected.

Recall that a 3-separation $(X, Y)$ of $M$ is a vertical 3-separation if $\min \{r(X), r(Y)\} \geq 3$. Following the terminology of Oxley, Semple, and Whittle [22], we say that a partition $(X,\{z\}, Y)$ is a vertical 3-separation of $M$ when $(X \cup\{z\}, Y)$ and $(X, Y \cup\{z\})$ are both vertical 3-separations and $z \in \operatorname{cl}(X) \cap \operatorname{cl}(Y)$.

We observe the following connection between elements that are $(N, B)$-robust but not $(N, B)$-strong and vertical 3-separations.

Lemma 2.2.2. [22. Lemma 3.1] Let $M$ be a 3-connected matroid, and e $\in E(M)$. If $\operatorname{si}(M / e)$ is not 3-connected, then $M$ has a vertical 3-separation $(X,\{e\}, Y)$.

We write "by orthogonality" to refer to an application of the next lemma.

Lemma 2.2.3. Let e be an element of a matroid $M$, and let $(X, e, Y)$ be a partition of $E(M)$. Then $e \in \operatorname{cl}(X)$ if and only if $e \notin \mathrm{cl}^{*}(Y)$.

The next three results state some elementary properties of 3-separations that we shall use implicitly. We use the notation $e \in \mathrm{cl}^{(*)}(X)$ to mean $e \in \operatorname{cl}(X)$ or $e \in$ $\operatorname{cl}^{*}(X)$.

Lemma 2.2.4. Let $X$ be an exactly 3-separating set in a 3-connected matroid, and suppose that $e \in E(M)-X$. Then $X \cup\{e\}$ is 3-separating if and only if $e \in \mathrm{cl}^{(*)}(X)$.

Lemma 2.2.5. Let $(X, Y)$ be an exactly 3-separating partition of a 3-connected matroid M. Suppose $|X| \geq 3$ and $x \in X$. Then

(i) $x \in \mathrm{cl}^{(*)}(X-\{x\})$; and 
(ii) $(X-\{x\}, Y \cup\{x\})$ is exactly 3-separating if and only if $x$ is in exactly one of $\operatorname{cl}(X-\{x\}) \cap \operatorname{cl}(Y)$ and $\operatorname{cl}^{*}(X-\{x\}) \cap \operatorname{cl}^{*}(Y)$.

Lemma 2.2.6. [1] Lemma 2.11] Let $(X, Y)$ be a 3-separation of a 3-connected matroid $M$. If $X \cap \operatorname{cl}(Y) \neq \emptyset$ and $X \cap \operatorname{cl}^{*}(Y) \neq \emptyset$, then $|X \cap \operatorname{cl}(Y)|=1$ and $\mid X \cap$ $\mathrm{cl}^{*}(Y) \mid=1$.

We use the phrase "by uncrossing" to refer to an application of the next Lemma.

Lemma 2.2.7. Let $M$ be a 3-connected matroid, and let $X$ and $Y$ be 3-separating subsets of $E(M)$. Then the following hold.

(i) If $|X \cap Y| \geq 2$, then $X \cup Y$ is 3-separating.

(ii) If $|E(M)-(X \cup Y)| \geq 2$, then $X \cap Y$ is 3-separating.

We will always be trying to keep an $N$-minor when removing elements. The next three results give some useful conditions for when we can keep an $N$-minor when dealing with 2-separations.

Lemma 2.2.8. [1] Lemma 4.3] Let $N$ be a 3-connected matroid such that $|E(N)| \geq$ 4. If $M$ has an $N$-minor, then $\mathrm{si}(M)$ has an $N$-minor.

Lemma 2.2.9. [22] Lemma 2.6] Let $e$ and $f$ be distinct elements of a 3-connected matroid $M$, and suppose that $\operatorname{si}(M / e)$ is 3-connected. Then either $M / e \backslash f$ is connected or $\operatorname{si}(M / e) \cong U_{2,3}$ and $M$ has no triangle containing $\{e, f\}$. Moreover, if no non-trivial parallel class of $M /$ e contains $f$, then $M / e / f$ is connected.

Lemma 2.2.10. [22, Lemma 2.7] Let $(X, Y)$ be a 2-separation of a connected matroid $M$ and let $N$ be a 3-connected minor of $M$. Then $\{X, Y\}$ has a member $S$ such that $|E(N) \cap S| \leq 1$. Moreover, if $s \in S$, then

(i) $M / s$ has an $N$-minor if $M / s$ is connected; and

(ii) $M \backslash s$ has an $N$-minor if $M \backslash s$ is connected.

If $(X,\{z\}, Y)$ is a vertical 3-separation of $M$, then it is easy to see that $M / z$ has a non-minimal 2-separation $(X, Y)$, that is, a 2-separation $(X, Y)$ such that $|X| \geq 3$ and $|Y| \geq 3$. If $M / z$ has a 3-connected minor $N$, then it follows from a well-known result [20, Proposition 8.3.5] that $|E(N) \cap X| \leq 1$ or $|E(N) \cap Y| \leq 1$. We refer to $X$ as the weak side of $(X,\{z\}, Y)$ if $|E(N) \cap X| \leq 1$. 
Lemma 2.2.11. [1] Lemma 4.5] Let $N$ be a 3-connected minor of a 3-connected matroid $M$. Let $(X,\{z\}, Y)$ be a vertical 3-separation of $M$ such that $M / z$ has an $N$-minor, where $|X \cap E(N)| \leq 1$. If $Y \cup\{z\}$ is closed, then there is at most one element of $X$ that is not $N$-flexible. Moreover, if such an element $x$ exists, then $x \in \mathrm{cl}^{*}(Y)$ and $z \in \operatorname{cl}(X-\{x\})$

We need the following routine upgrade of Lemma 2.2.11 that also covers the case when the " $N$-side" of the vertical 3-separation is not closed. It is an essential tool for dealing with elements that are $(N, B)$-robust but not $(N, B)$-strong.

Lemma 2.2.12. Let $N$ be a 3-connected minor of a 3-connected matroid $M$. Let $(X,\{z\}, Y)$ be a vertical 3-separation of $M$ such that $M / z$ has an $N$-minor, where $|X \cap E(N)| \leq 1$.

(i) If $Y \cup\{z\}$ is closed, then every element of $X$ is $N$-contractible and there is at most one element $x \in X$ that is not $N$-deletable. Moreover, if such an element $x$ exists, then $x \in \mathrm{cl}^{*}(Y)$ and $z \in \operatorname{cl}(X-\{x\})$.

(ii) If $Y \cup\{z\}$ is not closed, then every element of $X-\operatorname{cl}(Y)$ is $N$-contractible, and at most one element of $X$ is not $N$-deletable. Moreover, if such an element $x$ exists, then $x \in \mathrm{cl}^{*}(\operatorname{cl}(Y))$ and $z \in \operatorname{cl}(X-(\operatorname{cl}(Y) \cup x))$.

Proof. Suppose $Y \cup\{z\}$ is closed. Then (i) holds by Lemma 2.2.11. Assume that $Y \cup\{z\}$ is not closed, and let $s \in X \cap \operatorname{cl}(Y)$.

2.2.12.1. $\operatorname{si}(M / s)$ is not 3-connected.

Subproof. We prove that $(X-\{s\},\{s\}, Y \cup\{z\})$ is a vertical 3-separation of $M$. The partitions $(X, Y \cup\{z\})$ and $(X-\{s\}, Y \cup\{s, z\})$ are both vertical 3-separations of $M$ unless $r(X-\{s\})=2$. Suppose that $r(X-\{s\})=2$. But $s \in \operatorname{cl}(X-\{s\})$ because $X$ and $X-\{s\}$ are exactly 3 -separating, so $2=r(X-\{s\})=r(X)$, which contradicts the fact that $r(X) \geq 3$. Thus $(X-\{s\},\{s\}, Y \cup\{z\})$ is a vertical 3separation of $M$, and the claim follows.

Now $\operatorname{co}(M \backslash s)$ is 3-connected by 2.2.12.1 and Bixby's Lemma. Thus, by the dual of Lemma 2.2.9, the matroid $M \backslash s / z$ is connected. Now, by Lemma 2.2.10, $M \backslash s / z$ and hence $M \backslash s$ has an $N$-minor, so $s$ is $N$-deletable. 
Now, by taking the vertical 3-separation $(\operatorname{cl}(Y), z, X-\operatorname{cl}(Y))$ of $M$ and applying (i), it follows that (ii) holds.

The following result is an elementary consequence of orthogonality. We will primarily make use of this result, and its dual, for certifying that an element satisfies the connectivity requirement to be an $(N, B)$-strong element.

Lemma 2.2.13. Let $M$ be a 3-connected matroid. If $X$ is a rank-2 subset and $|X| \geq 4$, then $M \backslash x$ is 3-connected for all $x \in X$.

We employ the following results when we encounter fans.

Lemma 2.2.14. [1] Lemma 2.12] Let $M$ be a 3-connected matroid such that $|E(M)| \geq 7$. Suppose that $M$ has a fan $F$ of at least 4 elements, and let $f$ be an end of $F$.

(i) If $f$ is a spoke element, then $\operatorname{co}(M \backslash f)$ is 3-connected and $\operatorname{si}(M / f)$ is not 3-connected.

(ii) If $f$ is a rim element, then $\operatorname{si}(M / f)$ is 3-connected and $\operatorname{co}(M \backslash f)$ is not 3connected.

Lemma 2.2.15. Let $M$ be a matroid with distinct elements $f_{1}, f_{2}, f_{3}, f_{4}$. If the only triangle containing $f_{3}$ is $\left\{f_{1}, f_{2}, f_{3}\right\}$ and the only triad containing $f_{2}$ is $\left\{f_{2}, f_{3}, f_{4}\right\}$, then $\operatorname{si}\left(M / f_{3}\right) \cong \operatorname{co}\left(M \backslash f_{2}\right)$.

We conclude this section with some easy connectivity results about 2-separations. A 2-separation $(X, Y)$ of $M$ is minimal if $|X|=2$ or $|Y|=2$.

Lemma 2.2.16. Let $M$ be 3-connected and $e \in E(M)$. If $M \backslash$ e has a minimal 2separation $(X, Y)$, then $X$ or $Y$ is a series pair.

Proof. Let $(X, Y)$ be a minimal 2-separation of $M \backslash e$, and assume that $|X|=2$. Then $\lambda_{M \backslash e}(X)=r_{M \backslash e}(X)+r_{M \backslash e}^{*}(X)-2=1$, so $r_{M \backslash e}(X)+r_{M \backslash e}^{*}(X)=3$. Since $M \backslash e$ is connected, we have $\left\{r_{M \backslash e}(X), r_{M \backslash e}^{*}(X)\right\}=\{1,2\}$. Thus $X$ is a series pair or a parallel pair of $M \backslash e$. But $M$ is 3-connected, so $X$ is not a parallel pair. Therefore $X$ is a series pair. 
Lemma 2.2.17. Let $M$ be a 3-connected matroid, and e, $f \in E(M)$. Let $(X, Y)$ be a 2-separation of $M \backslash$ e. If $f \in X$ and $|X| \geq 3$, then $M \backslash e / f$ and $M \backslash e, f$ are not 3-connected.

Proof. Suppose that $f \in X$ and that $|X| \geq 3$. Now $(X-\{f\}, Y)$ is a partition of the ground set of both $M \backslash e / f$ and $M \backslash e, f$ such that $\min |X-\{f\}|,|Y| \geq 2$. Moreover, $\lambda_{M \backslash e / f} \leq \lambda_{M \backslash e}(X)=1$ and $\lambda_{M \backslash e, f} \leq \lambda_{M \backslash e}(X)=1$. Thus $(X-\{f\}, Y)$ is a 2-separation of both $M \backslash e / f$ and $M \backslash e, f$.

In particular, series pairs are important when we study $(N, B)$-strong elements in Section 2.4 .

Lemma 2.2.18. Let $M$ be a 3-connected matroid, and let $u \in E(M)$ be an element such that $\operatorname{co}(M \backslash u)$ is 3-connected. If $S$ and $S^{\prime}$ are distinct series classes of $M \backslash u$, then $S \cup S^{\prime}$ is independent.

Proof. Suppose $S \cup S^{\prime}$ is dependent in $M \backslash u$. Then there is a circuit $C$ of $M \backslash u$ that meets both $S$ and $S^{\prime}$. But then we can contract all but one element of each series class in such a way that $\operatorname{co}(M \backslash u)$ has a parallel pair; a contradiction.

Lemma 2.2.19. Let $M$ be a 3-connected matroid, and let $u \in E(M)$ be an element such that $\operatorname{co}(M \backslash u)$ is 3-connected. Let $S$ be a series class of $M \backslash u$ such that $|S| \geq 2$. If there is some element $s \in S$ such that $\operatorname{si}(M / s)$ is not 3-connected, then

(i) $S$ is a series pair of $M \backslash u$;

(ii) $M \backslash u$ has exactly two distinct series classes; and

(iii) $s^{\prime} \in S-\{s\}$ is an element such that $\operatorname{si}\left(M / s^{\prime}\right)$ is 3-connected.

Proof. Suppose that there is some element $s \in S$ such that $\operatorname{si}(M / s)$ is not 3connected. Then $S$ is a series pair by the dual of Lemma 2.2.13, so (i) holds. Now $M / s$ has a vertical 3-separation $(A, s, B)$, and we may assume that $u \in A$. Then $(A-u, B)$ is a 2-separation of $M / s \backslash u$, and this matroid is 3-connected up to series classes because $\operatorname{co}(M \backslash u)$ is 3 -connected. Thus $A-u$ is a series class of $M \backslash u$. Since $s \in \operatorname{cl}_{M}(A)$, there is a circuit $C$ of $M$ such that $s \in C \subseteq A \cup\{s\}$. Moreover, $u \in C$ because $S \cup(A-\{u\})$ is independent by Lemma 2.2.18. Suppose there is some series pair $S^{\prime}$ of $M \backslash u$ disjoint from $S \cup(A-\{u\})$. Then $S^{\prime} \cup\{u\}$ is a triad of $M$ that meets the circuit $C$ in the single element $u$; a contradiction 
to orthogonality. Thus $S$ and $A-u$ are the only series classes of $M \backslash u$, so (ii) holds. Finally, let $s^{\prime} \in S-\{s\}$ and suppose that $\operatorname{si}\left(M / s^{\prime}\right)$ is not 3-connected. Then there is some vertical 3-separation $\left(A^{\prime}, s^{\prime}, B^{\prime}\right)$ for $M$. Assume that $u \in A^{\prime}$. Then $\left(A^{\prime}-u, B^{\prime}\right)$ is a 2-separation of $M / s^{\prime} \backslash u$, and this matroid is 3-connected up to series classes because $\operatorname{co}(M \backslash u)$ is 3-connected. Thus $A^{\prime}-u$ is a series class of $M \backslash u$. By (ii), $A^{\prime}-\{u\}=A-\{u\}$, and thus $\left(A^{\prime}, s^{\prime}, B^{\prime}\right)=\left(A, s^{\prime}, B\right)$. But $s^{\prime} \notin \operatorname{cl}_{M}(A)$ because $S \cup(A-\{u\})$ is independent; a contradiction. Therefore (iii) holds.

\subsection{The setup}

This section contains preliminary material on excluded minors for the class of $\mathbb{P}$ representable matroids that is crucial to our approach. Partial field preliminaries and matrix notation can be found in Section 1.1 .

Let $N$ be a 3-connected non-binary matroid. A matroid $M$ with an $N$-minor is $N$-stable if, whenever $(X, Y)$ is a 2-separation of $M$ with $|X \cap E(N)| \leq 1$, then the matroid $M_{X}$ corresponding to $X$ in the 1- or 2-sum decomposition of $M$ induced by $(X, Y)$ is binary.

In this chapter, we assume that $M$ has a coindependent pair of elements $a, b$ such that $M \backslash a, b$ is 3-connected with an $N$-minor, where $N$ is a 3-connected strong $\mathbb{P}$-stabilizer. Thus $M \backslash a, M \backslash b$, and $M \backslash a, b$ are all $N$-stable.

Let $\mathbb{P}$ be a partial field, and let $M$ and $N$ be 3-connected $\mathbb{P}$-representable matroids such that $N$ is a minor of $M$. Suppose the ground set of $N$ is $X^{\prime} \cup Y^{\prime}$, where $X^{\prime}$ is a basis of $N$. Recall that $N$ is a $\mathbb{P}$-stabilizer for $M$ if, whenever $A_{1}$ and $A_{2}$ are $X \times Y$ $\mathbb{P}$-matrices (where $X^{\prime} \subseteq X$ and $Y^{\prime} \subseteq Y$ ) such that

(i) $M=M\left[I \mid A_{1}\right]=M\left[I \mid A_{2}\right]$;

(ii) $A_{1}\left[X^{\prime}, Y^{\prime}\right]$ is scaling-equivalent to $A_{2}\left[X^{\prime}, Y^{\prime}\right]$; and

(iii) $N=M\left[I \mid A_{1}\left[X^{\prime}, Y^{\prime}\right]\right]=M\left[I \mid A_{2}\left[X^{\prime}, Y^{\prime}\right]\right]$,

then $A_{1}$ is scaling-equivalent to $A_{2}$.

Let $\mathscr{M}$ be a class of matroids. Recall that $N$ is a $\mathbb{P}$-stabilizer for $\mathscr{M}$ if $N$ is a $\mathbb{P}$-stabilizer for every 3-connected $\mathbb{P}$-representable matroid $M \in \mathscr{M}$ with an $N$ minor. Recall that $N$ is a strong $\mathbb{P}$-stabilizer for $\mathscr{M}$ if $N$ is a $\mathbb{P}$-stabilizer for $\mathscr{M}$ 
and, for every 3-connected $\mathbb{P}$-representable matroid $M \in \mathscr{M}$ with an $N$-minor, every $\mathbb{P}$-representation of $N$ extends to a $\mathbb{P}$-representation of $M$. When we say that $N$ is a "strong $\mathbb{P}$-stabilizer" without reference to a class of matroids we mean the class of $\mathbb{P}$-representable matroids.

Let $B$ be a basis for a matroid $M$, and let $Z$ be a subset of $E(M)$. We write $M_{B}[Z]$ to denote the minor $M /(B-Z) \backslash\left(B^{*}-Z\right)$.

Theorem 2.3.1. [19] Theorem 5.5] Let $D$ be an $X_{N} \times Y_{N} \mathbb{P}$-matrix such that $N=$ $M[I \mid D]$. Choose $B, E_{N} \subseteq E$ such that $B$ is a basis of $M \backslash\{a, b\}, E_{N} \subseteq E-\{a, b\}$ is such that $M_{B}\left[E_{N}\right]=N$, and $X_{N} \subseteq B$. Suppose $M \backslash a$ and $M \backslash b$ are $\mathbb{P}$-representable. Then there exists an $B \times(E-B)$ matrix $A$ with entries in $\mathbb{P}$ such that

(i) $A-a$ and $A-b$ are $\mathbb{P}$-matrices;

(ii) $M[I \mid A-a]=M \backslash a$ and $M[I \mid A-b]=M \backslash b$;

(iii) $A\left[E_{N}\right]$ is scaling-equivalent to $D$.

Moreover, the matrix A is unique up to row and column scaling.

We call the matrix $A$ of Theorem 2.3.1 the companion matrix for $M$.

A companion matrix for an excluded minor contains a certificate of nonrepresentability over $\mathbb{P}$. Let $B$ be a basis of $M$, and let $A$ be a $B \times(E(M)-B)$ matrix with entires in $\mathbb{P}$. A set $Z \subseteq E(M)$ incriminates the pair $(M, A)$ if $A[Z]$ is square and one of the following holds:

(i) $\operatorname{det}(A[Z]) \notin \mathbb{P}$;

(ii) $\operatorname{det}(A[Z])=0$ but $B \triangle Z$ is a basis of $M$;

(iii) $\operatorname{det}(A[Z]) \neq 0$ but $B \triangle Z$ is dependent in $M$.

The next result follows immediately from the definition.

Lemma 2.3.2. Let $A$ be an $X \times Y \mathbb{P}$-matrix, where $X$ and $Y$ are disjoint, and $X \cup Y=E$. Exactly one of the following statements is true:

(i) $A$ is a $\mathbb{P}$-matrix and $M=M[I \mid A]$.

(ii) Some $Z \subseteq X \cup Y$ incriminates $(M, A)$. 
The next Theorem shows that there is some companion matrix $A$ for $M$ that has a 4-element incriminating set. Recall that by geometrically-equivalent we mean that two matrices are equivalent up to scaling rows and columns by non-zero entries of $\mathbb{P}$, permuting rows, permuting columns, and pivoting.

Theorem 2.3.3. [19]. Theorem 5.8] Suppose $A-u, A-v$ are $\mathbb{P}$-matrices, and $M \backslash u=M[I(A-u)], M \backslash v=M[I(A-v)]$. Suppose $Z \subseteq X \cup Y$ incriminates $(M, A)$. Then there is some $X^{\prime} \times Y^{\prime}$ matrix $A^{\prime}$, and $a, b \in X^{\prime}$, such that $u, v \in Y^{\prime}, A-u$ is geometrically-equivalent to $A^{\prime}-u, A-v$ is geometrically-equivalent to $A^{\prime}-v$, and $\{a, b, u, v\}$ incriminates $\left(M, A^{\prime}\right)$.

The following result is proved by Hall, Mayhew, and van Zwam in [12] (See Proposition 3.1 and 3.2).

Lemma 2.3.4. Let $M$ be a $\mathbb{P}$-representable matroid, and let $N$ be a 3-connected strong $\mathbb{P}$-stabilizer minor of $M$. If $M$ is an $N$-stable matroid, then $M$ is strongly $\mathbb{P}$-stabilized by $N$.

The next Lemma can be proved by a straightforward modification of the proof of Mayhew, Whittle, and van Zwam in [19, Theorem 5.12]. The conditions in [19, Theorem 5.12] (iv) and (v) are upgraded from " $M_{B}\left[Z_{1}\right]$ and $M_{B}\left[Z_{2}\right]$ are 3connected up to series-parallel classes" to " $M_{B}\left[Z_{1}\right]$ and $M_{B}\left[Z_{2}\right]$ are $N$-stable" using Lemma 2.3.4.

Lemma 2.3.5. Let $N$ be a strong stabilizer for the class of $\mathbb{P}$-representable matroids, and suppose that $C \subseteq E(M)$ is such that $M_{B}[C]$ is (strictly) $N$-fragile. If there exist subsets $Z, Z_{1}, Z_{2} \subseteq E(M)$ such that:

(i) $a \in Z_{1}-Z_{2}$ and $b \in Z_{2}-Z_{1}$;

(ii) $C \cup\{x, y\} \subseteq Z \subseteq Z_{1} \cap Z_{2}$;

(iii) $M_{B}[Z]$ is connected;

(iv) $M_{B}\left[Z_{1}\right]$ is N-stable;

(v) $M_{B}\left[Z_{2}\right]$ is $N$-stable;

(vi) $\{a, b, x, y\}$ incriminates $\left(M_{B}\left[Z_{1} \cup Z_{2}\right], A\left[Z_{1} \cup Z_{2}\right]\right)$;

then $M_{B}\left[Z_{1} \cup Z_{2}\right]$ is not strongly $\mathbb{P}$-stabilized by $N$. 
We use the phrase "allowable pivot" to refer to an application of the next two results.

Lemma 2.3.6. [19. Lemma 5.10] If $p \in\{x, y\}, q \in B^{*}-\{a, b\}$, and $A_{p q} \neq 0$, then $\{x, y, a, b\} \triangle\{p, q\}$ incriminates $\left(M, A^{p q}\right)$.

If the $x$ and $y$ entries are both zero or the $a$ and $b$ entries are both zero, then we can pivot without changing the incriminating set.

Lemma 2.3.7. [19. Lemma 5.11] If $p \in B-\{x, y\}, q \in B^{*}-\{a, b\}$ are such that $A_{p q} \neq 0$, and either $A_{p a}=A_{p b}=0$ or $A_{x q}=A_{y q}=0$, then $\{a, b, x, y\}$ incriminates $\left(M, A^{p q}\right)$.

The elements of the 4-element incriminating set $\{a, b, x, y\}$ for $(M, A)$ label a $2 \times 2$ submatrix $A[\{a, b, x, y\}]$ of $A$. We will refer to the next result by saying "the bad submatrix has no zero-entries."

Lemma 2.3.8. If $\{a, b, x, y\}$ is an incriminating set for the pair $(M, A)$, then $A_{i j} \neq$ 0 for $i \in\{x, y\}$ and $j \in\{a, b\}$.

Proof. First assume that $A_{x b}=0$. Then $\operatorname{det}(A[\{a, b, x, y\}]) \in \mathbb{P}$. Since $\{a, b, x, y\}$ incriminates the pair $(M, A)$, it follows that either:

(i) $B \triangle\{a, b, x, y\}$ is a basis of $M$ but dependent in $M[I \mid A]$; or

(ii) $B \triangle\{a, b, x, y\}$ is dependent in $M$ but a basis of $M[I \mid A]$.

Assume that (i) holds, so $B \triangle\{a, b, x, y\}$ is a basis, but $\operatorname{det}(A[\{a, b, x, y\}])=0$. Then, it follows from $\operatorname{det}(A[\{a, b, x, y\}])=A_{x a} \cdot A_{y b}=0$ and the property that nonzero elements of $\mathbb{P}$ are units, that $A_{x a}=0$ or $A_{y b}=0$. Suppose that $A_{x a}=0$. Let $B^{\prime}=B \triangle\{a, b, x, y\}$. Now $B$ and $B^{\prime}$ are bases of $M$ and $x \in B-B^{\prime}$, so by basis exchange there is some $z \in B^{\prime}-B=\{a, b\}$ such that $(B-x) \cup z$ is a basis of $M$; a contradiction because $M \backslash b=M[I \mid A-b], M \backslash a=M[I \mid A-a]$ and $A_{x a}=A_{x b}=0$, so both $(B-x) \cup a$ and $(B-x) \cup b$ are dependent in $M$. Thus $A_{x a} \neq 0$, and a similar argument using basis exchange on $B$ and $B^{\prime}$ shows that $A_{y b} \neq 0$; a contradiction.

Now assume that (ii) holds, so $B^{\prime}=B \triangle\{a, b, x, y\}$ is dependent in $M$, but $B^{\prime}$ is a basis of $M[I \mid A]$. Then it follows from $\operatorname{det}(A[\{a, b, x, y\}])=A_{x a} \cdot A_{y b} \neq 0$ that both $A_{x a} \neq 0$ and $A_{y b} \neq 0$. Now $M \backslash b=M[I \mid A-b]$ and $A_{x a} \neq 0$, so $(B-x) \cup a$ is a 
basis of $M$. Similarly $M \backslash a=M[I \mid A-a]$ and $A_{y b} \neq 0$, so $(B-y) \cup b$ is also a basis of $M$. Let $B_{1}=(B-x) \cup a$ and $B_{2}=(B-y) \cup b$. Then $x \in B_{2}-B_{1}$, so by basis exchange there is some $z \in B_{1}-B_{2}$ such that $\left(B_{2}-x\right) \cup z$ is a basis of $M$. But $B_{1}-B_{2}=\{a, y\}$, so then $\left(B_{2}-x\right) \cup z$ is either $(B-x) \cup b$ or $B^{\prime}$; a contradiction because both $(B-x) \cup b$ and $B^{\prime}$ are dependent in $M$.

Our setup is as follows.

(i) $N$ is a 3-connected strong $\mathbb{P}$-stabilizer for the class of $\mathbb{P}$-representable matroids such that $|E(N)| \geq 4$ and $N$ neither a wheel nor a whirl.

(ii) $M$ is an excluded minor for the class of $\mathbb{P}$-representable matroids, and $M$ has a pair of elements $a, b$ such that $M \backslash a, b$ is 3-connected with an $N$-minor.

(iii) $M$ has a $B \times B^{*}$ companion matrix $A$ and $\{a, b, x, y\}$ is an incriminating set for $(M, A)$.

\subsection{Strong elements}

In this section, we prove results about the $(N, B)$-strong elements of $M^{\prime}=M \backslash a, b$ outside of the special rows $x$ and $y$ of the 4-element incriminating set $\{a, b, x, y\}$ of $(M, A)$. The main result here is a proof that $M^{\prime}$ has at most two $(N, B)$-strong elements outside of $x$ and $y$. Moreover, any $(N, B)$-strong elements outside of $x$ and $y$ are in $B^{*}-\{a, b\}$.

Lemma 2.4.1. If $u$ is an $(N, B)$-strong element of $M^{\prime}$ such that $u \notin\{x, y\}$, then $u \notin B$.

Proof. Suppose that $u$ is an $(N, B)$-strong element of $M^{\prime}$ such that $u \notin\{x, y\}$ and that $u \in B$. Let $Z=E(M)-\{a, b, u\}, Z_{1}=E(M)-\{b, u\}$, and $Z_{2}=E(M)-\{a, u\}$ where $C=E(N)$. Then, by Lemma 2.3.5, the matroid $M / u$ is not strongly $\mathbb{P}$ stabilized by $N$. But $M \backslash a, b / u$, and hence $M / u$, is 3-connected up to parallel classes. Now it follows from Lemma 2.3.4 that $M / u$ is strongly $\mathbb{P}$-stabilized by $N$; a contradiciton.

Lemma 2.4.2. If $u$ is an $(N, B)$-strong element of $M^{\prime}$ such that $u \notin\{x, y\}$, then $M \backslash a, u$ or $M \backslash b, u$ is not $N$-stable. 
Proof. Suppose that $u$ is an $(N, B)$-strong element of $M^{\prime}$ such that $u \notin\{x, y\}$. Then $u \in B^{*}-\{a, b\}$ by Lemma 2.4.1. Suppose that both $M \backslash a, u$ and $M \backslash b, u$ are $N$ stable. Let $Z=E(M)-\{a, b, u\}, Z_{1}=E(M)-\{b, u\}$, and $Z_{2}=E(M)-\{a, u\}$ where $C=E(N)$. Then, by Lemma 2.3.5, the matroid $M \backslash u$ is not strongly $\mathbb{P}$ stabilized by $N$. Thus $M \backslash u$ is not $N$-stable. But then $M \backslash a, u$ or $M \backslash b, u$ is not $N$-stable by Lemma 2.4.3; a contradiction.

Suppose that a matroid $R$ is 3 -connected up to series classes. The only way to create a matroid $Q$ that is not $N$-stable by a one-element extension of $R$ by $a$ is to put $a$ in the span of a series class $S$ of $Q$ so that the $S \cup a$ component in the 2-sum decomposition of $Q$ contains a $U_{2,4}$-minor. We call such a series class $S$ unstable. Thus, if $M^{\prime}$ has an $(N, B)$-strong element $u$, then it follows from Lemma 2.4 .2 that $M \backslash u, a$ or $M \backslash u, b$ has an unstable series class. Note that we sometimes abuse terminology and say that the $(N, B)$-strong element $u$ has an unstable series class.

We use the following result to locate $a$ or $b$ when $M^{\prime}$ has a strong element outside of $\{x, y\}$.

Lemma 2.4.3. Let $\operatorname{co}\left(M^{\prime} \backslash u\right)$ be 3-connected with a 3-connected minor $N$. If $M \backslash u, b$ is not $N$-stable, then there is some series class $S$ of $M^{\prime} \backslash u$ such that $a \in \operatorname{cl}(S), a \notin \operatorname{cl}(\{s\})$ for all $s \in S$, and $a \notin \operatorname{cl}(E(M)-S)$.

Note that the converse of Lemma 2.4.3 is not true.

We now consider the size of an unstable series class.

Lemma 2.4.4. Let $u$ be an $(N, B)$-strong element of $M^{\prime}$ such that $u \notin\{x, y\}$. If $C$ is a cosegment of $M^{\prime}$ such that $|C| \geq 4$ and $u \in C$, then $|C|=4$ and $B \cap C=\{x, y\}$.

Proof. Suppose $C$ is a cosegment of $M^{\prime}$ such that $|C| \geq 4$ and $u \in C$. Then $\mid C \cap$ $B^{*} \mid \leq 2$ since $C$ is a corank 2 subset, so $|C \cap B| \geq|C|-2$. Suppose that there is some $c \in C$ that is a member of $B-\{x, y\}$. Then $M^{\prime} / c$ is 3-connected with an $N$-minor, so $c$ is an $(N, B)$-strong element; a contradiction to Lemma 2.4.1. We deduce that $|C|=4$ and that $B \cap C=\{x, y\}$.

We now show that unstable series classes must meet $\{x, y\}$. Moreover, it follows that up to allowable pivots, we can make an unstable series class contain $\{x, y\}$. 
Lemma 2.4.5. Let $u$ be an $(N, B)$-strong element of $M^{\prime}$ such that $u \notin\{x, y\}$, and let $S$ be an unstable series class of $M^{\prime} \backslash u$. Then $S \cap B \subseteq\{x, y\}$.

Proof. The result follows from Lemma 2.4.4 if $S$ is a series class with three elements, so we may assume that $S=\left\{s_{1}, s_{2}\right\}$ is a series pair. It follows from the codependence of $S$ that $S$ meets $B$. Seeking a contradiction, suppose that $\left\{s_{1}, s_{2}\right\} \cap(B-\{x, y\}) \neq \emptyset$. Assume that $s_{1} \in B-\{x, y\}$. Then $\operatorname{si}\left(M^{\prime} / s_{1}\right)$ is not 3-connected by Lemma 2.4.1. Then $\operatorname{si}\left(M^{\prime} / s_{2}\right)$ is 3-connected by Lemma 2.2.19. so it follows from Lemma 2.4.1 that $s_{2} \in B^{*}-\{a, b\}$. Now, if $A_{x s_{2}}=A_{y s_{2}}=0$, then a pivot on $A_{s_{1} s_{2}}$ is allowable, and the basis $B \triangle\left\{s_{1}, s_{2}\right\}$ has an element that contradicts Lemma 2.4.1. Thus we may assume that $A_{x s_{2}} \neq 0$. Then a pivot on $A_{x s_{2}}$ is an allowable pivot, and $\left\{s_{1}, s_{2}\right\} \subseteq B \triangle\left\{x, s_{2}\right\}$. Now $\left\{s_{1}, s_{2}\right\}$ is an unstable series pair, so $a \in \operatorname{cl}_{M \backslash b}\left(\left\{s_{1}, s_{2}\right\}\right)$. Hence $\left(A^{x s_{2}}\right)_{a w} \neq 0$ if and only if $w \in\left\{s_{1}, s_{2}\right\}$. But then $\left(A^{x s_{2}}\right)_{a y}=0$; a contradiction because $A_{a y}^{x s_{2}}$ is an entry of the bad submatrix. Thus $S \cap B \subseteq\{x, y\}$.

Next we prove that unstable series classes involving the same element (i.e. $a$ or $b$ ) are simple to handle: they must be contained in a 4-point cosegment of $M^{\prime}$.

Lemma 2.4.6. Let $u$ and $v$ be $(N, B)$-strong elements outside of $\{x, y\}$. If both $M \backslash u, b$ and $M \backslash v, b$ are not $N$-stable, then $\{u, v, x, y\}$ is a cosegment of $M^{\prime}$.

Proof. Suppose that both $M \backslash u, b$ and $M \backslash v, b$ are not $N$-stable, and let $S_{u}$ and $S_{v}$ be unstable series classes for $u$ and $v$ respectively. Suppose that $S_{u} \cup u$ is a cosegment of $M^{\prime}$. Then $S_{u} \cup u=\{u, t, x, y\}$ for some $t \in B^{*}$ by Lemma 2.4.4. We claim that $t=v$. Suppose not. Then we may assume by Lemma 2.4.5 that $S_{v}=\{x, s\}$ for some $s \in B^{*}-\{a, b\}$. But then $S_{u} \cup S_{v} \cup\{u, v\}$ is a corank-3 subset with $\{u, v, s, t\}$ contained in the cobasis $B^{*}$; a contradiction. Thus $S_{v}=\{x, y\}$, so $t=v$ by Lemma 2.4 .4

We may now assume that both $S_{u} \cup u$ and $S_{v} \cup v$ are triads. Suppose $S_{u} \cap S_{v}=$ $\emptyset$. Then $S_{v} \subseteq E(M)-S_{u}$, so $a \in \operatorname{cl}\left(E(M)-S_{u}\right)$; a contradiction because $a \notin$ $\operatorname{cl}\left(E(M)-S_{u}\right)$. Thus $S_{u} \cap S_{v} \neq \emptyset$. Suppose that $\left|S_{u} \cap S_{v}\right|=1$. Then, since $S_{u} \cup a$ and $S_{v} \cup a$ are triangles, it follows that $S_{u} \cup S_{v}$ is a triangle of $M^{\prime}$, and so $\{u, v\} \cup S_{u} \cup S_{v}$ is a 5-element fan of $M^{\prime}$ with rim ends $u, v$. But then $\operatorname{co}\left(M^{\prime} \backslash v\right)$ is not 3-connected; a contradiction because $v$ is an $(N, B)$-strong element. Therefore $S_{u}=S_{v}$. But then $\{u, v\} \cup S_{u}$ is a 4-point cosegment, so $S_{u} \cup\{u, v\}=\{u, v, x, y\}$ by Lemma 2.4 .4 
These results are enough to bound the number of $(N, B)$-strong elements outside of $\{x, y\}$. The bound on the number of $(N, B)$-strong elements is a key ingredient in many subsequent arguments.

Lemma 2.4.7. $M^{\prime}$ has at most two $(N, B)$-strong elements outside of $\{x, y\}$.

Proof. Seeking a contradiction, suppose that $p, q$, and $r$ are distinct $(N, B)$-strong elements of $M^{\prime}$ outside of $\{x, y\}$. Then $p, q, r \in B^{*}-\{a, b\}$ by Lemma 2.4.1. Then, by Lemma 2.4.2, we may assume that $M \backslash p, b$ and $M \backslash q, b$ are not $N$-stable. Then $\{p, q, x, y\}$ is a cosegment of $M^{\prime}$ by Lemma 2.4.6. Now, if $M \backslash r, b$ is not $N$-stable, then $\{p, r, x, y\}$ is a cosegment of $M^{\prime}$ by Lemma 2.4.6. Hence $\{p, q, r, x, y\}$ is a cosegment of $M^{\prime}$; a contradiction of Lemma 2.4.4. Thus $M \backslash r, a$ is not $N$-stable by Lemma 2.4.2. Let $S_{r}$ be an unstable series class for $r$. Now if $S_{r}$ contains $\{x, y\}$, then $\{p, q, r, x, y\}$ is a cosegment of $M^{\prime}$; a contradiction of Lemma 2.4.4. Thus, by Lemma 2.4.5, we may assume that $S_{r}=\{x, s\}$ for some $s \in B^{*}-\{a, b, p, q, r\}$. But then $\{p, q, x, y\} \cup S_{p}$ is a corank-3 subset containing the 4-element coindependent set $\{p, q, r, s\}$; a contradiction.

We say that a subset $G$ of $M^{\prime}$ is a gadget if $\{x, y\} \subseteq G$, there are triads $T$ and $T^{\prime}$ of $M^{\prime}$ such that $G=T \cup T^{\prime},\left|T \cap T^{\prime}\right| \in\{1,2\}$, and $G \subseteq \mathrm{cl}^{*}\left(G \cap B^{*}\right)$. If $\left|T \cap T^{\prime}\right|=1$, then $G \cap B^{*}$ has at least one $(N, B)$-strong element. We will see later that a gadget enables us to bound the number of rows and columns of the companion matrix that are obstacles to allowable pivots, and hence enables us to bound $|E(M)|$.

Now we seek to prove that we see either a gadget of $M^{\prime}$ or one $(N, B)$-strong element outside $\{x, y\}$.

Lemma 2.4.8. If $M^{\prime}$ has two $(N, B)$-strong elements outside of $\{x, y\}$, then either:

(i) $M^{\prime}$ has a gadget; or

(ii) there is a basis $B^{\prime}$ of $M^{\prime}$ and incriminating set $\left\{a, b, x^{\prime}, y^{\prime}\right\}$ for $\left(M, A^{\prime}\right)$ such that $M^{\prime}$ has at exactly one $\left(N, B^{\prime}\right)$-strong element $u$ outside of $\left\{x^{\prime}, y^{\prime}\right\}$, and $\left\{u, x^{\prime}, y^{\prime}\right\}$ is a triad of $M^{\prime}$.

Proof. Suppose that $u$ and $v$ are $(N, B)$-strong elements outside of $\{x, y\}$. If $(M \backslash u) \backslash b$ and $(M \backslash v) \backslash b$ are both not $N$-stable, then $\{u, v, x, y\}$ is a cosegment of $M^{\prime}$ by Lemma 2.4.6, so (i) holds. Thus we may assume that $(M \backslash u) \backslash a$ and $(M \backslash v) \backslash b$ 
are not $N$-stable, but $(M \backslash u) \backslash b$ and $(M \backslash v) \backslash a$ are $N$-stable. Let $S_{u}$ and $S_{v}$ be the unstable series classes for $u$ and $v$. We may also assume that both $S_{u} \cup u$ and $S_{v} \cup v$ are triads of $M^{\prime}$, or (i) holds by Lemma 2.4.4. Consider the corank of $S_{u} \cup S_{v} \cup\{u, v\}$. Suppose that the corank of $S_{u} \cup S_{v} \cup\{u, v\}$ is at least 4. Then the triads $S_{u} \cup u$ and $S_{v} \cup v$ are disjoint. Thus, by Lemma 2.4.5, we may assume that $S_{u}=\{s, x\}$ and $S_{v}=\{t, y\}$ for some $s, t \in B^{*}-\{u, v\}$. If $A_{y s} \neq 0$, then a pivot on $A_{y s}$ gives a cobasis $B^{*} \triangle\{s, y\}$ that contains a triad $S_{v} \cup v$; a contradiction. Thus $A_{y s}=0$. But then $s$ is spanned by $B-\{y\}$, and hence $b$ is spanned by $B-\{y\}$. Then $A_{b y}=0$; a contradiction because $A_{b y}$ is an entry of the bad submatrix. Thus the corank of $S_{u} \cup S_{v} \cup\{u, v\}$ is two or three.

Suppose that $S_{u} \cup S_{v} \cup\{u, v\}$ is a corank-3 subset. Then it follows from Lemma 2.4 .5 that the triads $S_{u} \cup u$ and $S_{v} \cup v$ cannot be disjoint, so $\left|\left(S_{u} \cup u\right) \cap\left(S_{v} \cup v\right)\right|=1$. We claim that (i) holds. Suppose, up to relabelling $u$ and $v$, that $S_{u} \subseteq B$. Then $S_{u}=\{x, y\}$ by Lemma 2.4.5. Now $S_{v} \neq\{x, y\}$ because $S_{u} \cup S_{v} \cup\{u, v\}$ is a corank3 subset, so we can assume that $S_{v}=\{x, s\}$ for some $s \in B^{*}-\{u\}$ by Lemma2.4.5. Thus $S_{u} \cup S_{v} \cup\{u, v\}$ is a gadget of $M^{\prime}$. We may therefore assume that neither $S_{u}$ nor $S_{v}$ is contained in $B$. Then, up to relabelling $x$ and $y$, it follows from the corank of $S_{u} \cup S_{v} \cup\{u, v\}$ that $x \in S_{u}$ and $y \in S_{v}$, so $S_{u} \cup S_{v} \cup\{u, v\}$ is a gadget of $M^{\prime}$.

Finally, suppose that $S_{u} \cup S_{v} \cup\{u, v\}$ is a corank-2 subset of $M^{\prime}$. Then we may assume, by Lemma 2.4.5. that $S_{u} \cup S_{v} \cup\{u, v\}=\{u, v, x\}$. Now a pivot on $A_{y v}$ is allowable by Lemma 2.4.5. Let $B^{\prime}=B \triangle\{v, y\}, x^{\prime}=x$, and $y^{\prime}=v$. Then $u$ is an $\left(N, B^{\prime}\right)$-strong element outside of $\left\{x^{\prime}, y^{\prime}\right\}$ and $\left\{u, x^{\prime}, y^{\prime}\right\}$ is a triad. If $y$ is not $\left(N, B^{\prime}\right)$-strong, then (ii) holds. Suppose that $y$ is an $\left(N, B^{\prime}\right)$-strong element of $M^{\prime}$. If $y$ is cospanned by $\left\{u, x^{\prime}, y^{\prime}\right\}$, then $M^{\prime}$ has a 4-point cosegment $\left\{u, y, x^{\prime}, y^{\prime}\right\}$, so (i) holds. Assume that $y$ is not cospanned by $\left\{u, x^{\prime}, y^{\prime}\right\}$. Let $S_{y}$ be an unstable series class for $y$. Then either $S_{y} \cup y$ is a 4-point cosegment, or $S_{y} \cup y$ is a triad such that the corank of $S_{u} \cup S_{y} \cup\{u, y\}$ is at least 3, so in either case (i) holds by the above argument.

After we have handled the gadget case, we use the following result.

Lemma 2.4.9. If $M^{\prime}$ does not have a gadget, then either:

(i) there is some basis $B^{\prime}$ of $M^{\prime}$ and incriminating set $\left\{a, b, x^{\prime}, y^{\prime}\right\}$ for $\left(M, A^{\prime}\right)$ such that $M^{\prime}$ has exactly one $\left(N, B^{\prime}\right)$-strong element $u$ outside of $\left\{x^{\prime}, y^{\prime}\right\}$, and $\left\{u, x^{\prime}, y^{\prime}\right\}$ is a triad of $M^{\prime}$; or 
(ii) there is no basis $B^{\prime}$ of $M^{\prime}$ and incriminating set $\left\{a, b, x^{\prime}, y^{\prime}\right\}$ for $\left(M, A^{\prime}\right)$ such that $M^{\prime}$ has an $(N, B)$-strong element outside of $\{x, y\}$.

Proof. Suppose that $M^{\prime}$ does not have a gadget. By Lemma 2.4.7 every basis $B$ of $M^{\prime}$ has at most two $(N, B)$-strong elements outside of $\{x, y\}$. Suppose $M^{\prime}$ has a basis $B$ such that there are two $(N, B)$-strong elements outside of $\{x, y\}$. Then (i) holds by Lemma 2.4.8(ii). Thus we may assume that every basis $B$ of $M^{\prime}$ has at most one $(N, B)$-strong element outside of $\{x, y\}$. Suppose that $u \in B^{*}$ is an $(N, B)$ strong element outside of $\{x, y\}$. Then $M^{\prime} \backslash u$ has an unstable series pair, and by Lemma 2.4.5 we may assume that $B$ is chosen such that $\{u, x, y\}$ is a triad.

\subsection{The gadget case}

Recall that a subset $G$ of $M^{\prime}$ is a gadget if $\{x, y\} \subseteq G$, there are triads $T$ and $T^{\prime}$ of $M^{\prime}$ such that $G=T \cup T,\left|T \cap T^{\prime}\right| \in\{1,2\}$, and $G \subseteq \mathrm{cl}^{*}\left(G \cap B^{*}\right)$. If $\left|T \cap T^{\prime}\right|=1$, then there is at least one $(N, B)$-strong deletable element in $G$.

In other words, a gadget is either a 4-point cosegment, or two triads that meet in a single element with the property that the subset $\{x, y\}$ of special rows is cospanned by the cobasis elements of $G$. In this section we suppose that $M^{\prime}$ has a gadget, and we prove that $|E(M)| \leq|E(N)|+16$.

We begin with the following restriction on the $(N, B)$-strong elements of $M^{\prime}$.

Lemma 2.5.1. If $M^{\prime}$ has a gadget $G$, then there are no $(N, B)$-strong elements outside of $G$.

Proof. Suppose $G$ is a cosegment $\{u, v, x, y\}$. Suppose $w$ is an $(N, B)$-strong element outside of $G$. Then $w \in B^{*}$ by Lemma 2.4.1. Then $w$ has an unstable series pair that meets $\{x, y\}$. By corank argument it must be $\{x, y\}$, and so $\{u, v, w, x, y\}$ is a cosegment of $M^{\prime}$; a contradiction of Lemma 2.4.4.

Suppose $G$ is two triads that meet in a single element and contain $\{x, y\}$, say $G=\{u, v, w, x, y\}$ where $\{u, v, w\} \subseteq B^{*}$. Suppose $t$ is an $(N, B)$-strong element outside of $\{x, y\}$. Then $t \in B^{*}$ by Lemma 2.4.1, and $M^{\prime} \backslash t$ has a series pair that meets $\{x, y\}$ by Lemma 2.4.5. Let $T=\{t, x, z\}$ be the triad of $M^{\prime}$ containing $t$ and meeting $\{x, y\}$. If $z \in G$, then $G \cup T$ has corank-3 but contains a 4-element subset $\{t, u, v, w\}$ of $B^{*}$; a contradiction. If $z \notin G$, then $z \in B^{*}$ by Lemma 2.4.5. 
But then $G \cup T$ has corank-4 but contains a 5-element subset $\{t, u, v, w, z\}$ of $B^{*}$; a contradiction.

The following results consider allowable pivots of $M^{\prime}$.

Lemma 2.5.2. Let $w \in B-\{x, y\}$. Then $A_{w z} \neq 0$ for some $z \in B^{*}-G$ if and only if $w \notin \mathrm{cl}^{*}\left(G \cap B^{*}\right)$.

Lemma 2.5.3. If $G$ is a gadget, then $A_{x z}=A_{y z}=0$ for all $z \in B^{*}-\{G\}$.

Proof. Since $\{x, y\} \subseteq \mathrm{cl}^{*}\left(G \cap B^{*}\right)$, it follows that $A_{x z}=A_{y z}=0$ for all $z \in B^{*}-$ $\{G\}$.

Lemma 2.5.4. If $A_{w z} \neq 0$ for some $w \in B-\{x, y\}$ and $z \in B^{*}-G$, then a pivot on $A_{w z}$ is allowable. Moreover, $G$ is a gadget relative to the basis $B \triangle\{w, z\}$.

Proof. Suppose that $A_{w z} \neq 0$ for some $w \in B-\{x, y\}$ and $z \in B^{*}-G$. Then the pivot on $A_{w z}$ is allowable by Lemma 2.5.3. Since $G \cap B=G \cap(B \triangle\{w, z\})$ and $G \cap B^{*}=G \cap\left(B^{*} \triangle\{w, z\}\right)$, the pivot preserves the property that $G$ is a gadget.

Next we show that a gadget, together with allowable pivots, imposes the following restrictions on the elements of $E\left(M^{\prime}\right)-G$.

Lemma 2.5.5. There is no element $z \in E\left(M^{\prime}\right)-G$ such that:

(i) z is $N$-deletable, $\operatorname{co}\left(M^{\prime} \backslash z\right)$ is 3-connected, and $z \notin \mathrm{cl}^{*}\left(G \cap B^{*}\right)$; or

(ii) z is $N$-contractible and $\operatorname{si}\left(M^{\prime} / z\right)$ is 3-connected.

Proof. Assume that (i) holds. Then $z$ is not $(N, B)$-strong by Lemma 2.5.1, so $z \in B-\{x, y\}$. Now by Lemma 2.5.2 there is some $w \in B^{*}-G$ such that $A_{z w} \neq 0$. Let $B^{\prime}=B \triangle\{w, z\}$. By Lemma 2.5.4, a pivot on $A_{z w}$ is allowable, and the $(N, B)$ strong elements in $G$ are $\left(N, B^{\prime}\right)$-strong. But now $z$ is an $\left(N, B^{\prime}\right)$-strong element, so $M^{\prime}$ has a gadget and an $\left(N, B^{\prime}\right)$-strong element outside of $G$; a contradiction of Lemma 2.5.1,

Assume that (ii) holds. Since $z \notin\{x, y\}$, it follows from Lemma 2.4.1 that $z \in$ $B^{*}-G$. Then $A_{x z}=A_{y z}=0$ by Lemma 2.5.3, so there is some $w \in B-\{x, y\}$ such that $A_{w z} \neq 0$ because $M^{\prime}$ has no loops. Then a pivot on $A_{w z}$ is allowable by Lemma 2.5.4. Now $z$ is an $(N, B \triangle\{w, z\})$-strong element in $(B \triangle\{w, z\})-\{x, y\}$; a contradiction of Lemma 2.4.1. 
We employ the following consequence of the Splitter Theorem.

A splitter sequence for $N$ in $M$ is a pair $\left((C, D),\left(x_{1}, \ldots, x_{n}\right)\right)$, where $(C, D)$ is a partition of a subset $C \cup D$ of $E(M)$, and $\left(x_{1}, \ldots, x_{n}\right)$ is an ordering of the elements of $C \cup D$ such that:

(i) $M / C \backslash D \cong N$; and

(ii) $M /\left(C \cap\left\{x_{1}, \ldots, x_{i}\right\}\right) \backslash\left(D \cap\left\{x_{1}, \ldots, x_{i}\right\}\right)$ is 3-connected for all $i \in\{1, \ldots, n\}$.

Given a splitter sequence for $N$ in $M$, the problem of bounding $|E(M)|$ by some function of $|E(N)|$ is reduced to bounding the size of $|C \cup D|$.

Note that we will abuse notation by simply refering to a sequence of elements $x_{1}, \ldots, x_{n}$ a splitter sequence.

The next result is a tool for dealing with elements of a splitter sequence that are outside of $G$. If such an element $z$ is $(N, B)$-robust, then it cannot be $(N, B)$-strong by Lemma 2.5.1, so there is a vertical 3 -separation associated with $z$. We show that such a vertical 3-separation has the following properties.

Lemma 2.5.6. Let $R$ be a 3-connected minor of a 3-connected matroid $Q$, and let $Z=\left(z_{1}, \ldots, z_{n}\right)$ be a splitter sequence ordering for $E(Q)-E(R)$. Let $z_{i}$ be a contractible element for some $i \in\{1, \ldots, n\}$. If $\mathrm{si}\left(Q / z_{i}\right)$ is not 3-connected, then there is a vertical 3-separation $\left(X, z_{i}, Y\right)$ such that $\left|Y-\left\{z_{1}, \ldots, z_{i-1}\right\}\right| \leq 1$, and there is at most one element of $Y$ that is not $N$-flexible in $Q$. Moreover, if $s \in Y$ is not $N$-flexible in $Q$, then s is $N$-contractible in $Q$ and $\operatorname{si}(Q / s)$ is 3-connected.

Proof. Suppose that $\operatorname{si}\left(Q / z_{i}\right)$ is not 3-connected. Then $Q$ has a vertical 3separation $\left(X, z_{i}, Y\right)$. We may assume that $|E(R) \cap Y| \leq 1$ and that $X \cup z_{i}$ is closed in $Q$. But $\left(X-\left\{z_{1}, \ldots, z_{i-1}\right\}, Y-\left\{z_{1}, \ldots, z_{i-1}\right\}\right)$ is not a 2-separation of the 3-connected matroid $Q / z_{i} / C \cap\left\{z_{1}, \ldots, z_{i-1}\right\} \backslash D \cap\left\{z_{1}, \ldots, z_{i-1}\right\}$, so $\mid Y-$ $\left\{z_{1}, \ldots, z_{i-1}\right\} \mid \leq 1$. The remaining properties follow immediately from Lemma 2.2 .11

Let $C \cup D$ be the elements of the splitter sequence $\left(z_{1}, \ldots, z_{n}\right)$ for $N$ in $M^{\prime}$, where $N \cong M^{\prime} / C \backslash D$. A key to bounding the size of $|C \cup D|$ is to bound the elements in $\mathrm{cl}^{*}(G)-G$.

We will bound the elements of $D$ in $\operatorname{cl}^{*}(G)-G$, but we first need the following Lemma for corank-3 gadgets. 
Lemma 2.5.7. Let $G$ be a corank-3 gadget of $M^{\prime}$. If $z^{\prime}, z^{\prime \prime} \in C \cup D$ are in $\mathrm{cl}^{*}(G)-$ $G$, then there is no partition $(X, Y)$ of $G \cup\left\{z^{\prime}, z^{\prime \prime}\right\}$ such that both $r^{*}(X)$ and $r^{*}(Y)$ are at most two.

Proof. Since $G$ is not a 4-point cosegment, $|G|=5$. Thus $\left|G \cup\left\{z^{\prime}, z^{\prime \prime}\right\}\right|=7$. Seeking a contradiction, suppose that $(X, Y)$ is a partition of $G \cup\left\{z^{\prime}, z^{\prime \prime}\right\}$ such that $\max \left\{r^{*}(X), r^{*}(Y)\right\} \leq 2$. We may assume that $|X| \geq 4$. Then $X$ is a cosegment that contains an element $z \in\left\{z^{\prime}, z^{\prime \prime}\right\}$. Then $z \in D$ by Lemma 2.5.5(ii). Suppose $\left\{z^{\prime}, z^{\prime \prime}\right\} \subseteq \mathrm{cl}^{*}(X)$. Then $z^{\prime}, z^{\prime \prime} \in D$, but they are in a cosegment $X \cup\left\{z^{\prime}, z^{\prime \prime}\right\}$ of $M^{\prime}$, so it follows that $z^{\prime}, z^{\prime \prime}$ are $N$-flexible. If $\operatorname{si}\left(M^{\prime} / z\right)$ is 3-connected for $z \in\left\{z^{\prime}, z^{\prime \prime}\right\}$, then we contradict Lemma 2.5.5(ii). Thus by Bixby's Lemma $z^{\prime}, z^{\prime \prime}$ are $(N, B)$-strong. But there is an $(N, B)$-strong element $u \in G \cap B^{*}$, so $M^{\prime}$ has at least three $(N, B)$ strong elements outside of $\{x, y\}$; a contradiction of Lemma 2.4.7. Thus $|X|=4$ and $u, z^{\prime \prime} \in Y$. Now $Y$ is a triad, and $\operatorname{cl}^{*}(Y)$ is a 4-point cosegment that contains the $N$-deletable element $u$. Thus $z^{\prime \prime}$ is $N$-contactible and $M / z^{\prime \prime}$ is 3-connected; a contradiction of Lemma 2.5.5(ii).

Lemma 2.5.8. There are at most two elements of $D$ that belong to $\mathrm{cl}^{*}(G)-G$.

Proof. Suppose that there are at least three elements $z, z^{\prime}, z^{\prime \prime} \in\left(\mathrm{cl}^{*}(G)-G\right) \cap D$. Then $z, z^{\prime}, z^{\prime \prime} \in B-\{x, y\}$. We may assume that $z$ comes after $z^{\prime}$ and $z^{\prime \prime}$ in the splitter sequence ordering.

Now if $G$ is a 4-point cosegment, then $z$ and $z^{\prime}$ are elements of the cosegment. Since $z$ is in a series class of $M^{\prime} \backslash z^{\prime}$, the element $z$ is $N$-contractible in $M^{\prime}$. But $M^{\prime} / z$ is 3-connected by Lemma 2.2.13, so $z^{\prime}$ is an $(N, B)$-strong element of $B-\{x, y\}$; a contradiction of Lemma 2.4.1.

Assume $G$ is not a 4-point cosegment, so $\mathrm{cl}^{*}(G)$ has corank three. We first show that $z$ is $N$-contractible in $M^{\prime}$. Suppose that $\left\{z, z^{\prime}, z^{\prime \prime}\right\}$ is a triad of $M^{\prime}$. Then $z$ is $N$-contractible since it is in a series pair of $M \backslash z^{\prime}$, and $M \backslash z^{\prime}$ has an $N$-minor since $z^{\prime}$ is $N$-deletable. We may therefore assume that $\left\{z, z^{\prime}, z^{\prime \prime}\right\}$ is not a triad of $M^{\prime}$. Then $\left\{z, z^{\prime}, z^{\prime \prime}\right\}$ is a cobasis for $\operatorname{cl}^{*}(G)$. As $M \backslash\left\{z, z^{\prime}, z^{\prime \prime}\right\}$ has an $N$-minor, it follows that the minor obtained from $M^{\prime}$ by deleting any cobasis for $\mathrm{cl}^{*}(G)$ and contracting the remaining elements has an $N$-minor. In particular, the element $z$ is $N$-contractible in the matroid $M^{\prime} \backslash\left(G \cap B^{*}\right)$, so it follows that $z$ is $N$-contractible in $M^{\prime}$.

Now, $z$ is an $N$-contractible element of $M^{\prime}$, so it follows from Lemma 2.5.5 (ii) that $\operatorname{si}\left(M^{\prime} / z\right)$ is not 3-connected. Then there is a vertical 3-separation $(X, z, Y)$ of 
$M^{\prime}$. But then $X$ or $Y$ must cospan $\mathrm{cl}^{*}(G)$ by Lemma 2.5.7. Assume $X$ cospans $\mathrm{cl}^{*}(G)$. Then $z \in \mathrm{cl}^{*}(X)$, and by the definition of a vertical 3-separation $z \in \operatorname{cl}(Y)$; a contradiction of orthogonality.

Now we work towards a proof that $D \subseteq \mathrm{cl}^{*}(G)$. We first need the following easy observation on the elements of $C$ that are $N$-flexible in $M^{\prime}$.

Lemma 2.5.9. If $c \in C-G$ and $c$ is $N$-deletable, then $c \in \mathrm{cl}^{*}(G)$.

Proof. Suppose that $c \in C-G$ and that $c$ is $N$-deletable. Then $\operatorname{si}\left(M^{\prime} / c\right)$ is not 3-connected by Lemma 2.5.5(ii), so co $\left(M^{\prime} \backslash c\right)$ is 3-connected by Bixby's Lemma. Now it follows from Lemma 2.5.5 (i) that $c \in \mathrm{cl}^{*}(G)$.

Lemma 2.5.10. $D \subseteq \mathrm{cl}^{*}(G)$.

Proof. Let $z_{i}$ be the first element of $D$ outside of $\mathrm{cl}^{*}(G)$. Let $Z^{\prime}=\left\{z_{1}, \ldots, z_{i-1}\right\}$. Since $z_{i}$ is $N$-deletable and $z_{i} \notin \mathrm{cl}^{*}(G)$, it follows from Lemma 2.5.5 (i) that $\operatorname{co}\left(M^{\prime} \backslash z_{i}\right)$ is not 3-connected. By Lemma 2.5.6 there is a vertical 3-separation $\left(X, z_{i}, Y\right)$ of $\left(M^{\prime}\right)^{*}$ such that at most one element of $Y$ is not in $Z^{\prime}$ and all such elements are $N$-flexible. Now if $z^{\prime} \in Y \cap Z^{\prime}$ and $z^{\prime} \in D$, then $z^{\prime} \in \mathrm{cl}^{*}(G)$ by the choice of $z_{i}$. If $z^{\prime} \in Y \cap Z^{\prime}$ and $z^{\prime} \in C$, then $z^{\prime}$ is also $N$-deletable by Lemma 2.5.6. so $z^{\prime} \in \mathrm{cl}^{*}(G)$ by Lemma 2.5.9. If $Y$ has an element $s$ that is not $N$-flexible, then $s$ is $N$-deletable and $\operatorname{co}\left(M^{\prime} \backslash s\right)$ is 3-connected, so by Lemma 2.5.5 (i) $s \in \operatorname{cl}^{*}(G)$. Thus every element of $Y$ is in $\operatorname{cl}^{*}(G)$. Now it follows from the definition of a vertical 3-separation in $\left(M^{\prime}\right)^{*}$ that $r^{*}(Y) \geq 3$. Thus $Y$ cospans $\mathrm{cl}^{*}(G)$, and so $\mathrm{cl}^{*}(Y)=\mathrm{cl}^{*}(G)$. But $z_{i} \in \mathrm{cl}^{*}(Y)$ because $\left(X, z_{i}, Y\right)$ is a vertical 3-separation in $\left(M^{\prime}\right)^{*}$, so $z_{i} \in \mathrm{cl}^{*}(G)$; a contradiction.

We can now show that $\left|\mathrm{cl}^{*}(G)\right| \leq 7$.

Lemma 2.5.11. There are at most two elements of $C \cup D$ in $\mathrm{cl}^{*}(G)-G$.

Proof. Suppose that there are three elements $p, q, r \in C \cup D$ such that $p, q, r \in$ $\mathrm{cl}^{*}(G)-G$. Now it follows from Lemma 2.5.8 that at least one of $p, q, r \in C$, so we may assume that $p \in C$. Now $\operatorname{si}\left(M^{\prime} / p\right)$ is not 3-connected by Lemma 2.5.5.(ii). Thus $p$ is not in a 4-point cosegment of $M^{\prime}$. In particular, it follows that $G$ is not a 4-point cosegment. Let $(X, p, Y)$ be a vertical 3-separation of $M^{\prime}$. Then by Lemma 2.5.7 we may assume that $X$ cospans $G \cup\{q, r\}$, and hence $p$. But then $p \in \operatorname{cl}^{*}(X)$ and $p \in \operatorname{cl}(Y)$; a contradiction of orthogonality. 
It remains to consider the elements outside of $\operatorname{cl}^{*}(G)$. We do not believe that the bound obtained in the next result is tight, and it should be possible to improve on this bound in future research.

Lemma 2.5.12. There are at most seven elements of $C$ that are not in $\mathrm{cl}^{*}(G)$.

Proof. Suppose that there are at least eight elements of $C-\operatorname{cl}^{*}(G)$, and let $p_{1}, \ldots, p_{8}$ be the first eight such elements. It follows from Lemma 2.5.5(ii) that there is some vertical 3-separation $\left(X_{i}, p_{i}, Y_{i}\right)$ of $M^{\prime}$ for each $i \in\{1, \ldots, 8\}$. Assume that $\left|Y_{i} \cap E(N)\right| \leq 1$ for $i \in\{1, \ldots, 8\}$.

2.5.12.1. There is some pair $i, j \in\{1, \ldots, 8\}$ such that $\left|Y_{i} \cap Y_{j}\right| \geq 2$.

Subproof. It follows from Lemma 2.5.5 and Lemma 2.5.6 that $Y_{i} \subseteq \mathrm{cl}^{*}(G)$ for all $i \in\{1, \ldots, 8\}$. By Lemma 2.5.11 $\left|\mathrm{cl}^{*}(G)\right| \leq 7$. We know that $\left|Y_{i}\right| \geq 3$ because $Y_{i}$ is a side of a vertical 3-separation of $M^{\prime}$. Moreover, if $\left|Y_{i}\right|=3$, then $Y_{i}$ is a triad of $M^{\prime}$.

Suppose that $\left|Y_{i}\right| \geq 5$ for some $i \in\{1, \ldots, 8\}$. Up to labels, we may assume that $\left|Y_{1}\right| \geq 5$. If $\left|Y_{1} \cap Y_{2}\right| \geq 2$, then $Y_{1}, Y_{2}$ is the desired pair. Otherwise $\left|Y_{1} \cap Y_{2}\right|=1$ and $Y_{1} \cup Y_{2}$ covers $\operatorname{cl}^{*}(G)$. But then either $\left|Y_{1} \cap Y_{3}\right| \geq 2$ or $\left|Y_{2} \cap Y_{3}\right| \geq 2$. We may therefore assume that $\left|Y_{i}\right| \leq 4$ for each $i \in\{1, \ldots, 8\}$.

Now suppose that there are at least two $i, j \in\{1, \ldots, 8\}$ such that $\left|Y_{i}\right|=\left|Y_{j}\right|=4$. Then, up to labels, we can assume that $\left|Y_{1}\right|=\left|Y_{2}\right|=4$. Then either $\left|Y_{1} \cap Y_{2}\right| \geq 2$, or

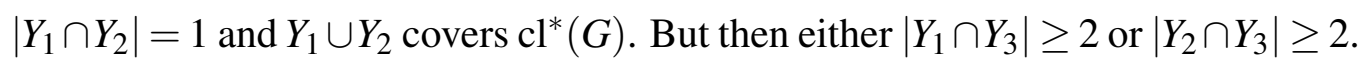
Thus we can assume that $\left|Y_{i}\right| \geq 4$ for at most one $i \in\{1, \ldots, 8\}$.

Assume that labels are chosen such that $Y_{1}, \ldots, Y_{7}$ are triads. Suppose that $\mid Y_{i} \cap$ $Y_{j} \mid \leq 1$ for all $i, j \in\{1, \ldots, 7\}$. We first consider the case that there are $i, j \in$ $\{1, \ldots, 8\}$ such that $Y_{i} \cap Y_{j}=\emptyset$. We may assume that labels are chosen such that $Y_{1} \cap Y_{2}=\emptyset$. Then it follows that $Y_{3} \cap Y_{4} \cap Y_{5} \neq \emptyset$. Let $y \in Y_{3} \cap Y_{4} \cap Y_{5}$. Now consider $Y_{6}$. It follows that $y \in Y_{6}$ or else $\left|Y_{i} \cap Y_{6}\right| \geq 2$ for some $i \in\{1,2\}$. But now $Y_{6}-\{y\}$ has at least two elements of $Y_{1} \cup Y_{2}$, and every element in $Y_{1} \cup Y_{2}$ is in $Y_{3} \cup Y_{4} \cup Y_{5}$. Thus $\left|Y_{i} \cap Y_{6}\right| \geq 2$ for some $i \in\{3,4,5\}$, which is a contradiction. We may therefore assume that $\left|Y_{i} \cap Y_{j}\right|=1$ for all $i, j \in\{1, \ldots, 7\}$. But then $\left|Y_{i} \cap Y_{8}\right| \geq$ 2 for some $i \in\{1, \ldots, 7\}$, and we have the desired pair.

Now let $Y_{i}$ and $Y_{j}$ be a pair such that $\left|Y_{i} \cap Y_{j}\right| \geq 2$. Now, by uncrossing, the sets $Y_{i} \cup Y_{j}, Y_{i} \cup Y_{j} \cup p_{i}, Y_{i} \cup Y_{j} \cup p_{j}, Y_{i} \cup Y_{j} \cup\left\{p_{i}, p_{j}\right\}$ are all 3-separating. Moreover, 
since $X_{i}$ and $X_{j}$ are the $N$-side, $\left|X_{i} \cap X_{j}\right| \geq 2$. Hence $Y_{i} \cup Y_{j}, Y_{i} \cup Y_{j} \cup p_{i}, Y_{i} \cup Y_{j} \cup p_{j}$, $Y_{i} \cup Y_{j} \cup\left\{p_{i}, p_{j}\right\}$ are sides of exact 3-separations of $M^{\prime}$ and $p_{i}, p_{j}$ are guts elements. Now $\left(X_{i} \cap X_{j}, p_{i}, Y_{i} \cup Y_{j} \cup p_{j}\right)$ is a vertical 3-separation of $M^{\prime}$ unless $r\left(X_{i} \cap X_{j}\right) \leq 2$. But if $r\left(X_{i} \cap X_{j}\right) \leq 2$, then $\left(X_{i} \cap X_{j}\right) \cup\left\{p_{i}, p_{j}\right\}$ is a 4-point segment of $M^{\prime}$. Hence $p_{i}$ or $p_{j}$ is $N$-deletable, so by Lemma 2.5.9 $p_{i}$ or $p_{j}$ is in $\mathrm{cl}^{*}(G)$; a contradiction. Thus $\left(X_{i} \cap X_{j}, p_{i}, Y_{i} \cup Y_{j} \cup p_{j}\right)$ is a vertical 3-separation of $M^{\prime}$, and $\mid\left(Y_{i} \cup Y_{j} \cup p_{j}\right) \cap$ $E(N) \mid \leq 1$. Then, by Lemma 2.2.12, either $\operatorname{si}\left(M^{\prime} / p_{j}\right)$ is 3-connected or $p_{j}$ is $N$-deletable in $M^{\prime}$, and so $p_{j} \in \mathrm{cl}^{*}(G)$ by Lemma 2.5.9; a contradiciton.

Finally, we are in position to prove the main result of this section.

Lemma 2.5.13. If $M^{\prime}$ has a gadget, then $|E(M)| \leq|E(N)|+16$.

Proof. Suppose that $M^{\prime}$ has a gadget $G$, and that $N=M^{\prime} / C \backslash D$ for some $C \cup D \subseteq$ $E(M)$. Now $D \subseteq \mathrm{cl}^{*}(G)$ by Lemma 2.5.10, and $\left|\mathrm{cl}^{*}(G)\right| \leq 7$ by Lemma 2.5.11. Moreover, $\left|C-\mathrm{cl}^{*}(G)\right| \leq 7$ by Lemma 2.5.12. Therefore $|E(M)| \leq|E(N)|+$ $\left|\mathrm{cl}^{*}(G)\right|+\left|C-\mathrm{cl}^{*}(G)\right|+|\{a, b\}|=|E(N)|+16$.

\subsection{Robust elements}

In this section, we consider the structure of $M^{\prime}$ that arises from elements that are $(N, B)$-robust but not $(N, B)$-strong. Recall that a path of 3-separations of $M^{\prime}$ is a partition $\left(P_{1}, \ldots, P_{n}\right)$ of $E\left(M^{\prime}\right)$ such that $\left(P_{1} \cup \cdots \cup P_{i}, P_{i+1} \cup \cdots \cup P_{n}\right)$ is a 3separation of $M^{\prime}$ for each $i \in\{1, \ldots, n-1\}$. The main result of this section will show that $(N, B)$-robust but not $(N, B)$-strong elements induce a natural path of 3-separations of $M^{\prime}$.

We assume that $M^{\prime}$ has no gadget. By Lemma 2.4.9 we may assume that the basis $B$ of $M^{\prime}$ is chosen such that either:

(i) There is some element $u \in B^{*}-\{a, b\}$ such that $u$ is an $(N, B)$-strong element and $\{u, x, y\}$ is a triad of $M^{\prime}$; or

(ii) there are no $(N, B)$-strong elements of $M^{\prime}$ outside of $\{x, y\}$.

If $B$ satisfies (ii), then we can assume there is no basis $B^{\prime}$ that satisfies (i). Furthermore, we assume that our basis $B$ is chosen to have the maximum number 
of $(N, B)$-robust elements outside of $\{x, y\}$ subject to (i) and (ii). In particular if we change the basis of $M^{\prime}$ from $B$ to $B^{\prime}$ by way of allowable pivots on elements of $E\left(M^{\prime}\right)-\{u, x, y\}$, then the number of $\left(N, B^{\prime}\right)$-robust elements of $M^{\prime}$ cannot be greater than the number of $(N, B)$-robust elements of $M^{\prime}$.

If $M^{\prime}$ has an $(N, B)$-strong element $u$ outside of $\{x, y\}$, then we let $S^{\prime}=\{u, x, y\}$. Otherwise we let $S^{\prime}=\{x, y\}$. Thus $S^{\prime}$ is the set of possible $(N, B)$-strong elements of $M^{\prime}$.

Let $(X,\{z\}, Y)$ be a vertical 3-separation of $M^{\prime}$. We say that $Y$ is $z$-closed if $Y$ is coclosed and $\operatorname{cl}(Y)-Y=\{z\}$. That is, $\mathrm{fcl}_{M / z}(Y)=Y$. We use $z$-closure to ensure that the $(N, B)$-strong elements of $M^{\prime}$ are contained in the weak side of a vertical 3-separation of $M^{\prime}$.

Lemma 2.6.1. If $z \in B$ and $z$ is $(N, B)$-robust but not $(N, B)$-strong, then there is some vertical 3-separation $(X,\{z\}, Y)$ of $M^{\prime}$ such that $Y$ is z-closed and $\mid Y \cap$ $E(N) \mid \leq 1$.

Proof. By Lemma 2.2.2 $M$ has a vertical 3-separation $(X, z, Y)$, and we may assume that $|Y \cap E(N)| \leq 1$. The elements of $\mathrm{fcl}_{M / z}(Y)-Y$ can be ordered $\left(y_{1}, \ldots, y_{m}\right)$ such that $Y \cup\left\{y_{1} \ldots, y_{i}\right\}$ is 2-separating for all $i \in\{1, \ldots, m\}$. Suppose for some $i$ that $Y_{i}=Y \cup\left\{y_{1} \ldots, y_{i}\right\}$ is the $N$-side. Let $j$ be the smallest index such that $\left|Y_{j} \cap E(N)\right|=2$. Then $\left(Y_{j}, X_{j}\right)=\left(Y \cup\left\{y_{1} \ldots, y_{j}\right\}, X-\left\{y_{1} \ldots, y_{j}\right\}\right)$ is a 2-separation of $M / z$ such that $\left|Y_{j} \cap E(N)\right|>1$ and $\left|X_{i} \cap E(N)\right|>1$; a contradiction. Thus $\left(Y_{i}, X_{i}\right)$ is a 2-separation such that $X_{i}$ is always the $N$-side for each $i$, so $\left|X_{i}\right| \geq 3$ for all $i$. Now $X_{m}$ is not a series or parallel class or we contradict the fact that $Y_{m}$ is fully closed. Thus $r_{M}\left(X_{i}\right) \geq 3$. Since $M$ is 3-connected, $z$ coblocks $\left(Y_{m}, X_{m}\right)$, and thus $\left(X_{m}, z, Y_{m}\right)$ is the desired vertical 3-separation.

Let $M$ be a matroid and $B$ a basis of $M$. A type-I fan relative to $B$ in $M$ is a 4-element fan $F$ with ordering $\left(f_{1}, f_{2}, f_{3}, f_{4}\right)$ where $\left\{f_{1}, f_{2}, f_{3}\right\}$ is a triangle and $F \cap B=\left\{f_{1}, f_{3}\right\}$. A type-II fan relative to $B$ in $M$ is a 4-element fan $F$ with ordering $\left(f_{1}, f_{2}, f_{3}, f_{4}\right)$ where $\left\{f_{1}, f_{2}, f_{3}\right\}$ is a triangle and $F \cap B=\left\{f_{1}, f_{3}, f_{4}\right\}$. A $P$-fan relative to $B$ is a type-I or type-II fan relative to $B$.

The next Lemma is contained in the proof of Lemma 3.4 and Lemma 4.8 of Brettell and Semple [1].

Lemma 2.6.2. Let $z \in B$ be an $(N, B)$-robust but not $(N, B)$-strong element of $M^{\prime}$, and let $(X,\{z\}, Y)$ be a $z$-closed vertical 3-separation of $M^{\prime}$. If there are not at 
least two distinct $(N, B)$-strong elements of $M^{\prime}$ contained in $Y$, then there is an $N$-contractible element $\alpha$, a vertical 3-separation $\left(X^{\prime},\{\alpha\}, Y^{\prime}\right)$ of $M^{\prime}$ such that $Y^{\prime} \cup\{\alpha\} \subseteq Y \cup\{z\}$, and a fan $F \subseteq Y^{\prime} \cup\{\alpha\}$ with ordering $(\alpha, \beta, \gamma, \delta)$ such that $F$ is a $P$-fan relative to $B$ and $\beta, \gamma$ are $N$-flexible.

We now prove that $S^{\prime} \subseteq Y$.

Lemma 2.6.3. If $z \in B-\{x, y\}, z$ is $(N, B)$-robust but not $(N, B)$-strong, and $(X,\{z\}, Y)$ is a $z$-closed vertical 3-separation of $M^{\prime}$, then $S^{\prime} \subseteq Y$.

Proof. Suppose that there are two distinct $(N, B)$-strong elements in $Y$. The $(N, B)$-strong elements of $M^{\prime}$ contained in $Y$ must belong to $S^{\prime}$. If $\left|S^{\prime}\right|=2$, then it follows immediately that $S^{\prime} \subseteq Y$. If $\left|S^{\prime}\right|=3$, then $S^{\prime}$ is a triad, so $S^{\prime} \subseteq Y$ because $Y$ is coclosed.

Thus we may assume that there are not two distinct $(N, B)$-strong elements in $Y$. Then it follows from Lemma 2.6.2 that there is an $N$-contractible element $\alpha$, a vertical 3-separation $\left(X^{\prime},\{\alpha\}, Y^{\prime}\right)$ of $M^{\prime}$ such that $Y^{\prime} \cup\{\alpha\} \subseteq Y \cup\{z\}$, and a fan $F \subseteq Y^{\prime} \cup\{\alpha\}$ with ordering $(\alpha, \beta, \gamma, \delta)$ such that $F$ is a $P$-fan relative to $B$, and $\beta, \gamma$ are $N$-flexible.

Note that $\alpha$ is not an $(N, B)$-strong element. But $\alpha$ is an $N$-deletable since $\alpha$ is in parallel with $\beta$ in $M^{\prime} / \gamma$ and $\gamma$ is $N$-contractible.

We need the following subproofs.

2.6.3.1. $\{x, y\} \cap\{\alpha, \gamma\} \neq \emptyset$

Subproof. Assume that $\{x, y\} \cap\{\alpha, \gamma\}=\emptyset$. Suppose $\beta$ is $(N, B)$-strong. Then, since $\beta \notin B$, it follows that $\{\beta, x, y\}$ is a triad. Now $\{\alpha, \beta, \gamma\}$ is a triangle that meets $\{\beta, x, y\}$, so by orthogonality $x$ or $y$ is in $\{\alpha, \gamma\}$; a contradiction to the assumption that $\{x, y\} \cap\{\alpha, \gamma\}=\emptyset$. Thus $\beta$ is not $(N, B)$-strong. But $\beta$ is $N$ flexible, so it follows that $\operatorname{co}\left(M^{\prime} \backslash \beta\right)$ is not 3-connected. Thus, by Bixby's Lemma, $\operatorname{si}\left(M^{\prime} / \beta\right)$ is 3-connected. Now $\{\alpha, \beta, \gamma\}$ is a triangle of $M^{\prime}$ and the cobasis element $\beta$ is spanned by the basis elements $\alpha$ and $\gamma$, so $A_{\alpha \beta} \neq 0$ and $A_{x \beta}=A_{y \beta}=0$. Thus a pivot on $A_{\alpha \beta}$ is an allowable pivot. But then $\beta$ is an $(N, B \triangle\{\alpha, \beta\})$-strong element outside of $\{x, y\}$ such that $\beta \in B \triangle\{\alpha, \beta\}$; a contradiction of Lemma 2.4.1. 
Thus $\alpha$ or $\gamma$ is a member of $\{x, y\}$. This implies that $\delta \in B^{*}$ because $\delta$ is $N$ contractible and $\operatorname{si}(M / \delta)$ is 3-connected by Lemma 2.2.14, so if $\delta \in B$, then $\delta \in$ $\{x, y\}$, so $\{x, y\} \subseteq F$. Hence $S^{\prime} \subseteq \mathrm{cl}^{*}(F)$.

We first handle the case when $\alpha \in\{x, y\}$.

2.6.3.2. If $\alpha \in\{x, y\}$, then $S^{\prime} \subseteq Y$.

Subproof. Assume that $\alpha=x$. Suppose that $\beta$ is $(N, B)$-strong. Then $\{\beta, x, y\}$ is a triad, so $S^{\prime} \subseteq \mathrm{cl}^{*}(F) \subseteq Y$, as required. Now suppose that $\beta$ is not $(N, B)$ strong. Consider the entry $A_{\alpha \beta}$. Since $\{\alpha, \beta, \gamma\}$ is a triangle of $M^{\prime}$ it follows that $A_{\alpha \beta} \neq 0$, so a pivot on $A_{\alpha \beta}$ is an allowable pivot. We then have a basis $B^{\prime}=B \triangle\{\alpha, \beta\}$ and incriminating set rows $\{\beta, y\}$ for $\left(M, A^{\alpha \beta}\right)$. Now, $\alpha$ is an $\left(N, B^{\prime}\right)$-strong element outside of $\{\beta, y\}$. Thus, by the choice of $B$, there is some element $u \in B^{*}$ such that $u$ is $(N, B)$-strong and $\{u, x, y\}$ is a triad. Since $\beta$ is not $(N, B)$-strong, $u \in E\left(M^{\prime}\right)-F$. But then $y \in\{\beta, \gamma\}$ by orthogonality, so $y=\gamma$. Therefore $S^{\prime} \subseteq \mathrm{cl}^{*}(F) \subseteq Y$.

Assume that $\alpha \notin\{x, y\}$. Then we may assume that $\gamma=x$. If $\beta$ is $(N, B)$-strong, then $\{\beta, x, y\}$ is a triad. But then $\{\beta, \delta, x, y\}$ is a 4-point cosegment; a contradiction because $M^{\prime}$ has no gadget. Thus we may assume that $\beta$ is not $(N, B)$-strong. Suppose that $\operatorname{co}\left(M^{\prime} \backslash x\right)$ is 3-connected. Now $A_{x \beta} \neq 0$ since $\{\alpha, \beta, x\}$ is a triangle of $M^{\prime}$, so a pivot on $A_{x \beta}$ is an allowable pivot. We then have a basis $B^{\prime}=B \triangle\{\gamma, \beta\}$ such that $x$ is $(N, B)$-strong element outside of $\{\beta, y\}$. By the choice of $B$, there is some $(N, B)$-strong element $u \in B^{*}$ such that $\{u, x, y\}$ is a triad. Since $u \notin F$, it follows from orthogonality that $\alpha=y$. Thus $S^{\prime} \subseteq \mathrm{cl}^{*}(F) \subseteq Y$.

We may therefore assume that $\operatorname{co}\left(M^{\prime} \backslash x\right)$ is not 3-connected. Then $\operatorname{si}\left(M^{\prime} / x\right)$ is 3 -connected by Bixby's Lemma. Since $\beta$ is not $(N, B)$-strong there is a vertical 3 -separation $(X, \beta, Y)$ of $\left(M^{\prime}\right)^{*}$. By orthogonality we may assume that $x \in X$ and $\alpha \in Y$. Consider $(X-x, x, Y \cup \beta)$. It cannot be a vertical 3-separation of $M^{\prime}$ since $\operatorname{si}\left(M^{\prime} / x\right)$ is 3 -connected. Thus $r(X-x) \leq 2$ and so $X$ contains a triangle. By orthogonality $X$ is a triangle and $X=\{x, \delta, \mu\}$ for some $\mu \in E\left(M^{\prime}\right)$. Moreover $\mu \in \operatorname{cl}(Y)$ or else $\{\beta, x, \delta, \mu\}$ is a 4-point cosegment; a contradiction of orthogonality. Thus $M^{\prime}$ has a 5 -point fan with ordering $(\alpha, \beta, x, \delta, \mu)$. Now $\operatorname{co}\left(M^{\prime} \backslash \mu\right)$ is 3-connected by Lemma 2.2 .14 and $\mu$ is $N$-deletable since $\mu$ is in a non-trivial parallel class in $M^{\prime} / x$. Now, if $\mu \in B^{*}-\{a, b\}$, then $\mu$ is $(N, B)$-strong and outside of $\{x, y\}$. Then $\{\mu, x, y\}$ is a triad. Then $\alpha=y$ by orthogonality; a contradiction 
to the assumption that $\alpha \notin\{x, y\}$. We may therefore assume that $\mu \in B$.

Now $\operatorname{co}\left(M^{\prime} \backslash x\right)$ is not 3-connected. Then there is a vertical 3-separation $(X, x, Y)$ of $\left(M^{\prime}\right)^{*}$. By orthogonality we may assume that $\alpha \in X$ and $\beta \in Y$. Consider the partition $(X \cup x, \beta, Y-\beta)$. Since $\operatorname{si}\left(M^{\prime} / \beta\right)$ is 3-connected by Bixby's Lemma, it follows that $(X \cup x, \beta, Y-\beta)$ cannot be a vertical 3-separation of $M^{\prime}$. Thus $r(Y-$ $\beta)=2$, so $Y$ is a triangle of $M^{\prime}$. By orthogonality $\mu \in X$ and $\delta \in Y$. Moreover $\varepsilon \in$ $\operatorname{cl}(X)$ or else $\{\varepsilon, x, \beta, \delta\}$ is a 4-point cosegment; a contradiction of orthogonality. Thus there is some element $\varepsilon \in E\left(M^{\prime}\right)$ such that $\{\alpha, \mu, \varepsilon\}$ is a triangle. But $\alpha, \mu \in$ $B$ and $\{\alpha, \mu, \varepsilon\}$ is a triangle, so it follows that $\varepsilon \in B^{*}$. We claim that $\varepsilon$ is an $(N, B)$-strong element of $M^{\prime}$. That $\varepsilon$ is $(N, B)$-robust follows from the fact that $\beta$ is $N$-contractible and $\{\delta, \varepsilon\}$ is a parallel pair in $M^{\prime} / \beta$. The fact that $\operatorname{co}\left(M^{\prime} \backslash \varepsilon\right)$ is 3-connected follows from Bixby's Lemma, since $\left(F, \varepsilon, E\left(M^{\prime}\right)-F\right)$ is a vertical 3-separation of $M^{\prime}$ so $\operatorname{si}\left(M^{\prime} / \varepsilon\right)$ is not 3-connected. Thus $\varepsilon$ is an $(N, B)$-strong element of $M^{\prime}$ outside of $\{x, y\}$. Thus $\{\varepsilon, x, y\}$ is a triad of $M^{\prime}$. But $\{x, y\}$ meets the triangle $\{\beta, \delta, \varepsilon\}$ in a single element; a contradiction of orthogonality.

We now work towards a proof that all of the $(N, B)$-robust elements are contained in the weak-side of a vertical 3-separation.

Lemma 2.6.4. Let $M$ be a 3-connected matroid and $(A, Z, B)$ a partition of $E(M)$ with $|A|,|B| \geq 2$. If, for all $z \in Z$, there is a path $\left(A^{\prime},\{z\}, B^{\prime}\right)$ of 3-separations such that $A \subseteq A^{\prime}$ and $B \subseteq B^{\prime}$, then there is an ordering $\left(z_{1}, \ldots, z_{n}\right)$ of the elements of $Z$ such that $\left(A, z_{1}, \ldots, z_{n}, B\right)$ is a path of 3-separations.

Proof. By induction on $|Z|=n$. If $n=1$, then the Lemma holds. Suppose that $n \geq 2$ and that the Lemma holds for all $m \leq n-1$. Let $z \in Z$. Then $M$ has a path of 3-separations $\left(A_{z}, z, B_{z}\right)$ such that $A \subseteq A_{z}$ and $B \subseteq B_{z}$.

2.6.4.1. $\left(A_{1}, Z_{1}, B_{1}\right)=\left(A, A_{z}-A, B_{z} \cup z\right)$ and $\left(A_{2}, Z_{2}, B_{2}\right)=\left(A_{z} \cup z, B_{z}-B, B\right)$ are paths of 3-separations of $M$ that satisfy the inductive hypotheses.

Subproof. It is clear that $\left(A_{1}, Z_{1}, B_{1}\right)$ is a partition of $E(M)$ and that $\left|Z_{1}\right|<|Z|$. It remains to prove that, for each $x \in Z_{1}$, there is a 3-path of the form $\left(A^{\prime \prime}, x, B^{\prime \prime}\right)$ such that $A_{1} \subseteq A^{\prime \prime}$ and $B_{1} \subseteq B^{\prime \prime}$. But there is a 3-path $\left(A_{x}, x, B_{x}\right)$ such that $A \subseteq A_{x}$ and $B \subseteq B_{x}$, so the result follows immediately if $z \in B_{x}$. We may therefore assume that $z \notin B_{x}$. Consider the partition $\left(A_{x} \cap A_{z}, x, B_{x} \cup B_{z} \cup z\right)$ of $M$. Then $A_{1} \subseteq A_{x} \cap A_{z}$ and $B_{1} \subseteq B_{x} \cup B_{z} \cup z$. It follows from uncrossing $B_{x}$ and $B_{z} \cup z$ that $B_{x} \cup B_{z} \cup z$ is 
3-separating, and by uncrossing $A_{x}$ and $A_{z}$ that $A_{x} \cap A_{z}$ is 3-separating. Thus the inductive hypotheses hold for $\left(A_{1}, Z_{1}, B_{1}\right)$. The same argument shows they also hold for $\left(A_{2}, Z_{2}, B_{2}\right)$.

It now follows from 2.6.4.1 and the induction assumption that there are paths of 3separations $\left(A_{1}, x_{1}, \ldots, x_{p}, B_{1}\right)$ and $\left(A_{2}, y_{1}, \ldots, y_{q}, B_{2}\right)$. Then concatenating these paths shows that $\left(A, x_{1}, \ldots, x_{p}, z, y_{1}, \ldots, y_{q}, B\right)$ is also a path of 3 -separations.

We observe that elements on the weak side of a vertical 3-separation that are not $N$-flexible are not $(N, B)$-robust in the following lemma.

Lemma 2.6.5. Let $z \in B-\{x, y\}$ be an element of $M^{\prime}$ that is $(N, B)$-robust but not $(N, B)$-strong, and let $(X,\{z\}, Y)$ be a vertical 3-separation of $M^{\prime}$ such that $|E(N) \cap Y| \leq 1$ and $S^{\prime} \subseteq Y$. If $\mu \in Y-S^{\prime}$ and $\mu$ is not $N$-flexible, then $\mu$ is not $(N, B)$-robust. Moreover, there are at most two such elements, and if $p$ and $q$ are two such elements, then $p \in \operatorname{cl}(X)$ and $q \in \mathrm{cl}^{*}(\operatorname{cl}(X))$.

Proof. Suppose that $\mu \in Y-S^{\prime}$ and $\mu$ is not $N$-flexible.

Assume that $\mu \in \operatorname{cl}(X)$. Then $\mu$ is in the guts of the 3 -separation $(X \cup\{z\}, Y)$, so the element $\mu$ is $N$-deletable. Suppose that $\mu$ is $(N, B)$-robust, so $\mu \in B^{*}-\{a, b\}$. But $(X, \mu,(Y-\mu) \cup z)$ is a vertical 3-separation of $M^{\prime}$, so it follows by Bixby's Lemma that $\operatorname{co}\left(M^{\prime} \backslash \mu\right)$ is 3-connected. Thus $\mu$ is an $(N, B)$-strong element, which is a contradiction because $\mu \notin S^{\prime}$. Therefore $\mu$ is not $(N, B)$-robust, so $\mu \in B$. Thus there can be at most one such element $\mu$ because $\{z, \mu\}$ spans the guts of the 3-separation $(X, Y \cup z)$.

We may therefore assume that $X \cup z$ is closed. Then it follows from Lemma 2.2.12 that $\mu$ is in the coguts of $(X \cup\{z\}, Y)$, and the element $\mu$ is not $N$-deletable but $\mu$ is $N$-contractible. Moreover, $\mu$ is the only element of $Y$ that is not $N$-flexible. Seeking a contradiction, assume that $\mu$ is an $(N, B)$-robust element. Then $\mu \in$ $B$, and $\operatorname{co}\left(M^{\prime} \backslash \mu\right)$ is 3-connected by Bixby's Lemma and the fact that $M^{\prime}$ has no $(N, B)$-strong elements in $B-\{x, y\}$. In particular, it follows that the partition $(X \cup z, \mu, Y-\mu)$ is not a vertical 3-separation of $\left(M^{\prime}\right)^{*}$, so $Y$ is a triad of $M^{\prime}$. But then $Y \cup z=\{x, y, z, \mu\}$ is contained in $B$, and $Y \cup z$ contains a circuit of $M^{\prime}$ because $z \in \operatorname{cl}(Y)$; a contradiction.

Let $\left(X^{\prime},\{z\}, Y^{\prime}\right)$ be a vertical 3-separation such that $Y^{\prime}$ is $z$-closed. Let $Y$ be the 3-separating set obtained from $Y^{\prime}$ by removing any elements of $Y^{\prime}-S^{\prime}$ that are not 
$(N, B)$-robust, and let $(X,\{z\}, Y)$ be the corresponding vertical 3-separation. We say that $(X,\{z\}, Y)$ is the good separation for $z$. Note we are moving at most two elements from one side of the partition to the other. Since $\left(X^{\prime},\{z\}, Y^{\prime}\right)$ is unique, and we move at most two elements, the good separation for $z$ is also unique.

The idea here is that, for each $(N, B)$-robust element of $M^{\prime}$, there is a good separation. All of the elements on the weak side of the good separation are $N$-flexible, and therefore $(N, B)$-robust. We now show that these separations induce a path in $M^{\prime}$.

Lemma 2.6.6. If $z_{1}, z_{2} \notin S^{\prime}$ are $(N, B)$-robust but not $(N, B)$-strong elements of $M^{\prime}$, and $\left(X_{1},\left\{z_{1}\right\}, Y_{1}\right),\left(X_{2},\left\{z_{2}\right\}, Y_{2}\right)$ are the good separations for $z_{1}$ and $z_{2}$ respectively, then $\left(X_{1}, Y_{1} \cup\left\{z_{1}\right\}\right)$ and $\left(X_{2}, Y_{2} \cup\left\{z_{2}\right\}\right)$ are nested separations.

Proof. Suppose that $\left(X_{1}, Y_{1} \cup\left\{z_{1}\right\}\right)$ and $\left(X_{2}, Y_{2} \cup\left\{z_{2}\right\}\right)$ cross. Now $\left|X_{1} \cap X_{2}\right| \geq 2$ since $\left|Y_{i} \cap E(N)\right| \leq 1$ for $i \in\{1,2\}$, and $\left|Y_{1} \cup Y_{2}\right| \geq 2$ since $S^{\prime} \subseteq Y_{1} \cup Y_{2}$. By uncrossing $Y_{1}$ and $Y_{2}$ it follows that $Y_{1} \cup Y_{2}$ is exactly 3-separating. Further uncrossing arguments show that $Y_{1} \cup Y_{2} \cup z_{1}, Y_{1} \cup Y_{2} \cup z_{2}$, and $Y_{1} \cup Y_{2} \cup\left\{z_{1}, z_{2}\right\}$ are also exactly 3 -separating.

Thus, up to duality, the partition $\left(X_{1} \cap X_{2}, Y_{1} \cup Y_{2} \cup\left\{z_{1}, z_{2}\right\}\right)$ is exactly 3-separating, $z_{2} \in B-\{x, y\}$ and $z_{2} \in \operatorname{cl}\left(X_{1} \cap X_{2}\right) \cap \operatorname{cl}\left(Y_{1} \cup Y_{2} \cup z_{1}\right)$.

Now $r\left(Y_{1} \cup Y_{2} \cup z_{1}\right) \geq r\left(Y_{1}\right) \geq 3$ since rank is non-decreasing on supersets.

2.6.6.1. $r\left(X_{1} \cap X_{2}\right) \geq 3$

Subproof. Suppose $r\left(X_{1} \cap X_{2}\right) \leq 2$. Then $z_{2} \in \operatorname{cl}\left(X_{1} \cap X_{2}\right)$, so $\left(X_{1} \cap X_{2}\right) \cup z_{2}$ is a segment with at least 3 -elements. If there are at least 3 elements of $X_{1} \cap X_{2}$, then there is some $(N, B)$-strong element in $X_{1} \cap X_{2}$; a contradiction because all $(N, B)$-strong elements belong to $S^{\prime} \subseteq Y_{1} \cup Y_{2} \cup\left\{z_{1}, z_{2}\right\}$. Thus $\left|X_{1} \cap X_{2}\right|=2$. But $z_{1} \in \mathrm{cl}^{(*)}\left(X_{1} \cap X_{2}\right)$ too, and if $z_{1} \in \operatorname{cl}\left(X_{1} \cap X_{2}\right)$, then $\left(X_{1} \cap X_{2}\right) \cup\left\{z_{1}, z_{2}\right\}$ is a 4element segment, so again there is some element of $X_{1} \cap X_{2}$ that is $(N, B)$-strong; a contradiction. Thus $z_{1} \in \mathrm{cl}^{*}\left(X_{1} \cap X_{2}\right)$, and so by orthogonality $\left(X_{1} \cap X_{2}\right) \cup\left\{z_{1}, z_{2}\right\}$ is a 4-element fan. Now, in the dual $\left(M^{\prime}\right)^{*}$, the partition $\left(\left(X_{1} \cap X_{2}\right) \cup z_{2}, z_{1}, Y_{1} \cup Y_{2}\right)$ is a vertical 3-separation for $z_{1}$, and $Y_{1} \cup Y_{2} \cup z_{1}$ contains $Y_{1} \cup\left\{z_{1}\right\}$, so $Y_{1} \cup Y_{2} \cup z_{1}$ and $Y_{1} \cup\left\{z_{1}\right\}$ are equal by the maximality of the good separation for $z_{1}$. But then $\left(X_{1} \cap X_{2}\right) \cup z_{2}$ is the $N$-side of the 2-separation of $M^{\prime} \backslash z_{1}$, so $N$ must be isomorphic to a minor of $U_{2,3}$; a contradiction. Thus $r\left(X_{1} \cap X_{2}\right) \geq 3$. 
Thus $\left(X_{1} \cap X_{2}, z_{2}, Y_{1} \cup Y_{2} \cup z_{1}\right)$ is a vertical 3-separation for $z_{2}$, and $Y_{1} \cup Y_{2} \cup z_{1}$ properly contains $Y_{2}$ by the assumption that $\left(X_{1}, Y_{1} \cup\left\{z_{1}\right\}\right)$ and $\left(X_{2}, Y_{2} \cup\left\{z_{2}\right\}\right)$ cross; a contradiction of the maximality of $Y_{2}$.

By Lemma 2.6.6, the weak sides of good separations are linearly ordered. Thus there is an $(N, B)$-robust element whose good separation is maximal.

Lemma 2.6.7. Let $Z$ be the set of elements of $E\left(M^{\prime}\right)-S^{\prime}$ that are $(N, B)$-robust but not $(N, B)$-strong. There is an ordering $\left(z_{1}, \ldots, z_{n}\right)$ of $Z$ such that $\left(X, z_{n}, \ldots, z_{1}, S^{\prime}\right)$ is a path of 3-separations of $M$, the elements of $\left\{z_{n-1}, \ldots, z_{1}\right\} \cup S^{\prime}$ are $N$-flexible, and $\left|E(N) \cap\left(\left\{z_{i}, \ldots, z_{1}\right\} \cup S^{\prime}\right)\right| \leq 1$ for all $i \in\{1, \ldots, n\}$.

Proof. By Lemma 2.6.5 for each $z \in Z$, there is a good separation $(X, z, Y)$ of $M$ where $|E(N) \cap Y| \leq 1, S^{\prime} \subseteq Y$, and all the elements of $Y$ are $N$-flexible. By Lemma 2.6.6 the good separations are nested, so there is some $z \in Z$ and a good separation $(X, z, Y)$ of $M$ such that $Z \subseteq Y$. Now applying Lemma 2.6.4 to the partition $\left(X, Z, S^{\prime}\right)$ of $M^{\prime}$, it follows that $Z$ has an ordering such that $\left(X, z_{n}, \ldots, z_{1}, S^{\prime}\right)$ is a path of 3-separations of $M^{\prime}$, as required.

\subsection{The main results}

Let $Z$ be the set of elements of $M^{\prime}$ that are $(N, B)$-robust but not $(N, B)$-strong. We first assume that $|Z| \geq 2$. Then by Lemma 2.6.7 there is an ordering $z_{1}, \ldots, z_{n}$ of $Z$ such that $\left(X, z_{n}, \ldots, z_{1}, S^{\prime}\right)$ is a path of 3-separations of $M^{\prime}$. Moreover, all elements of $\left\{z_{n-1}, \ldots, z_{1}\right\} \cup S^{\prime}$ are $N$-flexible. In this section we study this path of 3 -separations, and use it to prove the main results.

Recall that $\left|S^{\prime}\right| \in\{2,3\}$. In the case where $\left|S^{\prime}\right|=3$, we shall let $z_{1}$ label the $(N, B)$ strong element outside of $\{x, y\}$ and relabel the elements of $Z$ accordingly, so that we can always write the path of 3 -separations as $\left(X, z_{n}, \ldots, z_{1},\{x, y\}\right)$.

Let $(P, Q)$ be a 3-separation. Recall that $\operatorname{cl}(P) \cap \operatorname{cl}(Q)$ is the guts of $(P, Q)$ and that $\mathrm{cl}^{*}(P) \cap \mathrm{cl}^{*}(Q)$ is the coguts of $(P, Q)$. We say that an element $z_{i} \in Z$ is a guts or coguts element according to whether $z_{i}$ is in the guts or coguts of the 3-separation $\left(X \cup\left\{z_{n}, \ldots, z_{i}\right\},\left\{z_{i-1}, \ldots, z_{1}\right\} \cup\{x, y\}\right)$.

Lemma 2.7.1. $\left\{x, y, z_{1}\right\}$ is not a triangle. 
Proof. If $z_{1} \in S^{\prime}$, then $S^{\prime}=\left\{x, y, z_{1}\right\}$ and $S^{\prime}$ is a triad, so $\left\{x, y, z_{1}\right\}$ is not a triangle since $M^{\prime}$ is 3-connected. We may therefore assume that $z_{1} \notin S^{\prime}$. Suppose that $\left\{x, y, z_{1}\right\}$ is a triangle. Now, if $z_{1} \in B$, then the triangle $\left\{x, y, z_{1}\right\}$ is contained in the basis $B$; a contradiction. Thus $z_{1} \in B^{*}$, and $z_{1}$ is $N$-deletable because $z_{1}$ is $(N, B)$-robust. But $\operatorname{co}\left(M^{\prime} \backslash z_{1}\right)$ is not 3-connected because $z_{1}$ is not an $(N, B)$-strong element, so there is a vertical 3-separation $\left(R, z_{1}, G\right)$ of $\left(M^{\prime}\right)^{*}$ where $R$ is the $N$ side. By orthogonality and the fact that $\left\{x, y, z_{1}\right\}$ is a triangle, we can assume that $x \in R$ and $y \in G$. It follows by Lemma 2.2.12 that $y$ is $N$-flexible. Hence $x$ and $y$ are $N$-deletable, because $y$ is $N$-flexible and $\left\{x, y, z_{1}\right\}$ is a triangle of $M^{\prime}$.

Now $x$ and $y$ are $N$-deletable, so $\operatorname{co}\left(M^{\prime} \backslash x\right)$ and $\operatorname{co}\left(M^{\prime} \backslash y\right)$ are not 3-connected because $B$ is a robust basis so no allowable pivot using the $x$ or $y$ row can produce a basis $B^{\prime}$ with an $\left(N, B^{\prime}\right)$-strong element in $E\left(M^{\prime}\right)-\left(B^{\prime} \cup\{a, b\}\right)$. Then there is a vertical 3-separation $(P, x, Q)$ of $\left(M^{\prime}\right)^{*}$ and we may assume that $y \in P$ and $z_{1} \in Q$ because $\left\{x, y, z_{1}\right\}$ is a triangle of $M$. But then either $(P-y, y, Q \cup x)$ or $\left(P \cup x, z_{1}, Q-z_{1}\right)$ is a vertical 3-separation of $M$, so either $\operatorname{si}\left(M^{\prime} / y\right)$ or $\operatorname{si}\left(M^{\prime} / z_{1}\right)$ is not 3-connected, which is a contradiction of Bixby's Lemma. Thus $\left\{x, y, z_{1}\right\}$ is not a triangle.

Lemma 2.7.2. $z_{1} \in B^{*}$.

Proof. The statement is clearly true if $z_{1}$ is an $(N, B)$-strong element of $M^{\prime}$. Suppose that $M^{\prime}$ has no $(N, B)$-strong elements and that $z_{1} \in B$. Then $\operatorname{si}\left(M^{\prime} / z_{1}\right)$ is not 3-connected because $M^{\prime}$ has no $(N, B)$-strong elements in $B-\{x, y\}$. Thus $M^{\prime}$ has a vertical 3-separation $\left(P, z_{1}, Q\right)$ where $P$ is the $N$-side. Since $\left\{z_{1}, x, y\right\}$ is a triad, we may assume that $x \in P$ and $y \in Q$. By Lemma 2.2.12 there is at most one element of $Q$ that is not $N$-flexible, and hence $(N, B)$-robust, so in particular $M^{\prime}$ has another $(N, B)$-robust element $z_{2}$. Now, if $z_{2}$ is in the coclosure of $\left\{z_{1}, x, y\right\}$, then $\left\{x, y, z_{1}, z_{2}\right\}$ is a 4-element cosegment of $M^{\prime}$, so $M^{\prime} / z_{1}$ is 3-connected, which is a contradiction. Thus $z_{2}$ is in the closure of $\left\{z_{1}, x, y\right\}$ and $\left(E\left(M^{\prime}\right)-\left\{x, y, z_{1}, z_{2}\right\}, z_{2},\left\{x, y, z_{1}\right\}\right)$ is a vertical 3-separation of $M^{\prime}$. Thus $\operatorname{si}\left(M^{\prime} / z_{2}\right)$ is not 3-connected. Now $\left\{z_{1}, x, y\right\} \subseteq B$ and $\left\{z_{1}, x, y\right\}$ spans $z_{2}$, so it follows that $z_{2} \in B^{*}$. But then $z_{2}$ is $N$-deletable, and $\operatorname{co}\left(M^{\prime} \backslash z_{2}\right)$ is 3-connected by Bixby's Lemma, so $z_{2}$ is an $(N, B)$-strong element of $M^{\prime}$, which is a contradiction. Thus $z_{1} \in B^{*}$.

Lemma 2.7.3. Let $z \in Z-\{x, y\}$. Then $z$ is a guts element if and only if $z \in B$. 
Proof. Suppose that $z$ is a guts element. Then, by Lemma 2.7.1 and Lemma 2.7.2, we may assume that $z=z_{i}$ for some $i \geq 2$. If $z$ is not $N$-deletable, then $z \in B$ because $z$ is an $(N, B)$-robust element. Thus we may assume that $z$ is $N$-deletable. Since $z$ is in the guts of a vertical 3-separation, it follows that $\operatorname{co}\left(M^{\prime} \backslash z\right)$ is 3connected by Bixby's Lemma, so $z \in B$ because $z$ is not an $(N, B)$-strong element outside of $S^{\prime}$.

Conversely, suppose that $z$ is a coguts element. By Lemma 2.7.2 we may assume that $z=z_{i}$ for some $i \geq 2$. If $z$ is not $N$-contractible, then $z \in B^{*}$ because $z$ is an $(N, B)$-robust element. Thus we may assume that $z$ is $N$-contractible. Now $z$ is in the guts of a vertical 3-separation of $\left(M^{\prime}\right)^{*}$, so it follows from Bixby's Lemma that $\operatorname{si}\left(M^{\prime} / z\right)$ is 3 -connected. Thus $z \in B^{*}$ because $M^{\prime}$ has no $(N, B)$-strong elements in $B-\{x, y\}$.

We need the following result from Whittle and Williams [38].

Lemma 2.7.4. [38. Lemma 2.13] Let $M$ be a 3-connected matroid with a triad $\{a, b, c\}$ and circuit $\{a, b, c, d\}$. Then at least one of the following holds.

(i) $\operatorname{co}(M \backslash a)$ or $\operatorname{co}(M \backslash c)$ is 3-connected.

(ii) There exist $a^{\prime}, c^{\prime} \in E(M)$ such that both $\left\{a, a^{\prime}, c\right\}$ and $\left\{b, c, c^{\prime}\right\}$ are triangles.

(iii) There exists $f \in E(M)$ such that $\{a, b, c, f\}$ is a cosegment.

We can now handle the case when $n \geq 2$. Note that we believe that the conditions that appear in the following results of this section which involve bounding the rank or corank of $M$ as a function of the rank or corank of $N$ can be improved on in future research.

Lemma 2.7.5. If $n \geq 2$, then $r(M) \leq r(N)+5$ or $|E(M)| \leq|E(N)|+8$.

Proof. Suppose that $n \geq 2$. Then, since $\left\{x, y, z_{1}\right\}$ is 3-separating and not a triangle, it follows that $\left\{x, y, z_{1}\right\}$ is a triad of $M^{\prime}$.

2.7.5.1. $z_{1} \in B^{*}$.

Subproof. Suppose that $z_{1} \in B$. Then $z_{1}$ is a guts element, so there is a circuit that meets the triad $\left\{x, y, z_{1}\right\}$ in the single element $z_{1}$; a contradiction of orthogonality. 
Now consider the element $z_{2} \in Z$.

2.7.5.2. $z_{2} \in B$.

Subproof. Suppose $z_{2} \notin B$. Then $\left\{x, y, z_{1}, z_{2}\right\}$ is a 4-element cosegment; a contradiction because $M^{\prime}$ has no gadget. Thus $z_{2} \in B$.

Now $\left\{x, y, z_{1}, z_{2}\right\}$ is either a 4-element fan, or a 4-element circuit.

2.7.5.3. $\operatorname{cl}\left(\left\{x, y, z_{1}\right\}\right)-\left\{x, y, z_{1}\right\}=\left\{z_{2}\right\}$.

Subproof. Suppose there is some $z \notin\left\{x, y, z_{1}, z_{2}\right\}$ such that $z \in \operatorname{cl}\left(\left\{x, y, z_{1}\right\}\right)$. If $z \notin \operatorname{cl}\left(E\left(M^{\prime}\right)-\left\{x, y, z_{1}, z_{2}\right\}\right)$, then $z \in \operatorname{cl}^{*}\left(\left\{x, y, z_{1}\right\}\right)$, and so $\left\{x, y, z_{1}, z\right\}$ is a 4-point cosegment; a contradiction because $M^{\prime}$ has no gadget. Thus $z$ is in the guts of the vertical 3-separation for $z_{2}$. By Lemma 2.2.12 the element $z$ is $N$-deletable and $\operatorname{co}\left(M^{\prime} \backslash z\right)$ is 3-connected. Now, if $z \in B^{*}$, then $z$ is an $(N, B)$-strong element outside of $S^{\prime}$; a contradiction. Thus $z \in B$. But then the subset $\left\{x, y, z, z_{2}\right\} \subseteq B$ is independent and contained in the rank-3 subset $\mathrm{cl}\left(\left\{x, y, z_{1}\right\}\right)$; a contradiction.

The following claim is the key step for enabling allowable pivots.

2.7.5.4. $A_{z a}=A_{z b}=0$ for all $z \in B-\left\{x, y, z_{2}\right\}$.

Subproof. Suppose $\left\{x, y, z_{1}, z_{2}\right\}$ is a 4-element circuit. Now, since $M^{\prime}$ has no gadget and $\left\{x, y, z_{1}, z_{2}\right\}$ is closed by 2.7.5.3, it follows from Lemma 2.7.4 that $\operatorname{co}\left(M^{\prime} \backslash x\right)$ or $\operatorname{co}\left(M^{\prime} \backslash y\right)$ is 3-connected. Assume that $\operatorname{co}\left(M^{\prime} \backslash x\right)$ is 3-connected. Now $A_{x z_{1}} \neq 0$ because $\left\{x, y, z_{1}, z_{2}\right\}$ is a circuit, so a pivot on $A_{x z_{1}}$ is allowable. Let $B^{\prime}=B \triangle\left\{x, z_{1}\right\}$. Then $x$ is an $\left(N, B^{\prime}\right)$-strong element outside of $\left\{z_{1}, y\right\}$. Thus $x$ has an unstable pair that meets $\left\{z_{1}, y\right\}$ by Lemma 2.4.5, so $\left\{z_{1}, y\right\}$ is the unstable series pair for $x$ and $b \in \operatorname{cl}\left(\left\{y, z_{1}\right\}\right)$. By the choice of $B$ it follows that $z_{1}$ is $(N, B)$-strong outside of $\{x, y\}$ and that $a \in \operatorname{cl}(\{x, y\})$. Since $z_{1} \in \operatorname{cl}\left(\left\{x, y, z_{2}\right\}\right)$, it follows that $b \in \operatorname{cl}\left(\left\{x, y, z_{2}\right\}\right)$. Now $a, b \in \operatorname{cl}\left(\left\{x, y, z_{2}\right\}\right)$, so $A_{z a}=A_{z b}=0$ for all $z \in B-\left\{x, y, z_{2}\right\}$.

We may therefore assume that $\left\{x, y, z_{1}, z_{2}\right\}$ is a 4-element fan with ordering $\left(z_{2}, z_{1}, x, y\right)$ where $\left\{z_{2}, z_{1}, x\right\}$ is a triangle. Suppose that $\operatorname{co}\left(M^{\prime} \backslash x\right)$ is not 3connected. Then there is a vertical 3-separation $(W, x, X)$ of $\left(M^{\prime}\right)^{*}$. By orthogonality we may assume that $z_{1} \in W$ and that $z_{2} \in X$. Now $\left(W-z_{1}, z_{1}, X \cup x\right)$ is not a vertical 3-separation of $M^{\prime}$ because $\operatorname{si}\left(M^{\prime} \backslash z_{1}\right)$ is 3-connected, so $r\left(W-z_{1}\right)=2$. 
Thus $W$ is a triangle of $M^{\prime}$ containing $z_{1}$. By orthogonality $y \in W$ too, so there is an element in $\operatorname{cl}\left(\left\{y, z_{1}\right\}\right)-\left\{x, y, z_{1}, z_{2}\right\}$; a contradiction of 2.7.5.3. Thus $\operatorname{co}\left(M^{\prime} \backslash x\right)$ is 3-connected. Now $A_{x z_{1}} \neq 0$ because $z_{1}$ is a triangle with $x$, so a pivot on $A_{x z_{1}}$ is allowable. Let $B^{\prime}=B \triangle\left\{x, z_{1}\right\}$. Then $x$ is an $\left(N, B^{\prime}\right)$-strong element outside of $\left\{z_{1}, y\right\}$. Thus $x$ has an unstable pair that meets $\left\{z_{1}, y\right\}$ by Lemma 2.4.5, so $\left\{z_{1}, y\right\}$ is the unstable series pair for $x$ and we may assume that $b \in \operatorname{cl}\left(\left\{y, z_{1}\right\}\right)$. By the choice of $B$ it follows that $z_{1}$ is $(N, B)$-strong outside of $\{x, y\}$ and that $a \in \operatorname{cl}(\{x, y\})$. Since $z_{1} \in \operatorname{cl}\left(\left\{x, y, z_{2}\right\}\right)$, it follows that $b \in \operatorname{cl}\left(\left\{x, y, z_{2}\right\}\right)$. Now $a, b \in \operatorname{cl}\left(\left\{x, y, z_{2}\right\}\right)$, so $A_{z a}=A_{z b}=0$ for all $z \in B-\left\{x, y, z_{2}\right\}$.

Now we can can show that the elements outside of $\left\{x, y, z_{1}, z_{2}\right\}$ are $N$-essential.

2.7.5.5. If $N \cong M^{\prime} / C \backslash D$, then $r(M) \leq r(N)+5$ or $\left|(C \cup D)-\left\{x, y, z_{1}, z_{2}\right\}\right| \leq 2$.

Subproof. Suppose that $N \cong M^{\prime} / C \backslash D$ for some subsets $C$ and $D$ of $E\left(M^{\prime}\right)$. Suppose that $r(M) \geq r(N)+6$ and $\left|(C \cup D)-\left\{x, y, z_{1}, z_{2}\right\}\right| \geq 3$. We claim that at most one element of $(C \cup D)-\left\{x, y, z_{1}, z_{2}\right\}$ is an $(N, B)$-robust element of $M^{\prime}$.

Suppose that $w_{1}, w_{2}$ are $(N, B)$-robust elements of $(C \cup D)-\left\{x, y, z_{1}, z_{2}\right\}$. Then we may assume that $w_{1}=w$ is $N$-flexible in $M^{\prime}$. Suppose $w \in B$. Then $\operatorname{si}\left(M^{\prime} / w\right)$ is not 3-connected by Lemma 2.4.1, so co $\left(M^{\prime} \backslash w\right)$ is 3-connected by Bixby's Lemma. Now there is some $z \in B^{*}-\left\{z_{1}\right\}$ such that $A_{w z} \neq 0$ because $w$ is not a coloop of $M^{\prime}$. A pivot on $A_{w z}$ is allowable by 2.7.5.4. But then $w$ is an $\left(N, B^{\prime}\right)$-strong element outside of $\operatorname{cl}^{*}\left(\left\{z_{1}, x, y\right\}\right)$, so $M^{\prime}$ has a gadget; a contradiction. Suppose $w \in B^{*}$. Then $w$ is $N$-contractible, and $\operatorname{si}\left(M^{\prime} / z\right)$ is 3-connected by Bixby's Lemma. By 2.7.5.3 $z$ is not spanned by $\left\{x, y, z_{2}\right\}$, so there is some $z \in B-\left\{x, y, z_{2}\right\}$ such that $A_{z w} \neq 0$. A pivot on $A_{z w}$ is allowable by 2.7.5.4. Then $B^{\prime}=B \triangle\left\{z, z^{\prime}\right\}$ has an $\left(N, B^{\prime}\right)$-strong element in $B-\{x, y\}$; a contradiction of Lemma 2.4.1.

Thus at most one element of $(C \cup D)-\left\{x, y, z_{1}, z_{2}\right\}$ is an $(N, B)$-robust element. Now since $r(M) \geq r(N)+6$ it follows that there are $w, w^{\prime} \in B$ such that $w, w^{\prime} \in(C \cup$ $D)-\left\{x, y, z_{1}, z_{2}\right\}$ and $w, w^{\prime}$ are not $(N, B)$-robust. Then $w, w^{\prime}$ are $N$-deletable, and at least one of $w, w^{\prime}$ has at least two elements $s, t \in B^{*}-\left\{a, b, z_{1}\right\}$ such that $A_{w s} \neq 0$ and $A_{w t} \neq 0$ because $w$ is not a coloop of $M$, and $M^{\prime} \backslash z_{1}$ has at most two series pairs. At least one of $s, t$ is not an $(N, B)$-robust element of $(C \cup D)-\left\{x, y, z_{1}, z_{2}\right\}$ because there is at most one such element. Then a pivot on $A_{w s}$ is allowable, and $B \triangle\{w, s\}$ is a basis with more $(N, B)$-robust elements than $B$; a contradiction of the choice of $B$. 
Now $N \cong M^{\prime} / C \backslash D$ for some $C \cup D \subseteq E\left(M^{\prime}\right)$, and $r(M) \leq r(N)+5$ or $|C \cup D| \leq 6$ by 2.7.5.5, so $\left|E\left(M^{\prime}\right)\right| \leq|E(N)|+6$. Thus $r(M) \leq r(N)+5$ or $|E(M)| \leq|E(N)|+$ 8.

Thus, if Theorem 2.1.1 (a) does not hold for $M$, that is, if $r(M) \geq r(N)+6$ and $|E(M)| \geq|E(N)|+9$, then $M$ has at most one $(N, B)$-robust element outside of $\{x, y\}$. The case when $M^{\prime}$ has no $(N, B)$-robust elements outside of $\{x, y\}$ is handled next.

Lemma 2.7.6. If $M^{\prime}$ has no $(N, B)$-robust elements outside of $\{x, y\}$, then $M \backslash a, b$ is $N$-fragile.

Proof. The elements outside of $\{x, y\}$ are not $(N, B)$-robust. Thus the elements of $B-\{x, y\}$ are not $N$-contractible, and the elements of $B^{*}-\{a, b\}$ are not $N$ deletable. Thus it remains to show that $M^{\prime} \backslash x$ and $M^{\prime} \backslash y$ have no $N$-minor. Suppose that $x$ is $N$-deletable. There is some $x^{\prime} \in B^{*}-\{a, b\}$ such that $A_{x x^{\prime}} \neq 0$ because $x$ is not a coloop of $M^{\prime}$. Then perform an allowable pivot on $A_{x x^{\prime}}$. Let $B^{\prime}=B \triangle\left\{x, x^{\prime}\right\}$. Now $x \in\left(B^{\prime}\right)^{*}-\{a, b\}$, so $x$ is an $\left(N, B^{\prime}\right)$-robust element outside of $\left\{x^{\prime}, y\right\}$; a contradiction because $B$ was chosen to have the most $(N, B)$-robust elements outside of $\{x, y\}$. Thus $x$ is not $N$-deletable. A similar argument shows that $y$ is not $N$-deletable.

We can now prove the first main theorem.

Theorem 2.7.7. Let $M$ be an excluded minor for the class of $\mathbb{P}$-representable matroids, and let $N$ be a strong $\mathbb{P}$-stabilizer. If $M$ has a pair of elements $a, b$ such that $M \backslash a, b$ is 3-connected with an $N$-minor, then at least one of the following holds:

(a) $|E(M)| \leq|E(N)|+16$ or $r(M) \leq r(N)+5$; or

(b) there is some robust basis B for $M$ such that either:

(i) $M \backslash a, b$ is $N$-fragile, and $M \backslash a, b$ has at most one $(N, B)$-robust element $z \in B^{*}-\{a, b\}$ outside of $\{x, y\}$. Moreover, if $z \in B^{*}-\{a, b\}$ is $(N, B)$ robust, then $z$ is an $(N, B)$-strong element of $M \backslash a, b$, and $\{x, y, z\}$ is a triad of $M \backslash a, b$; or 
(ii) $M \backslash a, b$ is not $N$-fragile, but the only $N$-flexible elements of $M \backslash a, b$ are contained in a triad $\{x, y, z\}$ of $M \backslash a, b$ for some $(N, B)$-strong $z \in B^{*}$. Moreover, the only $(N, B)$-robust elements of $M \backslash a, b$ are in $\{x, y, z\}$, and $M$ has a cocircuit $\{p, x, y, z\}$ or $\{a, b, x, y, z\}$ that contains a triangle $\{p, x, y\}$ for some $p \in\{a, b\}$.

Proof. It follows from Lemma 2.4.9 that $M^{\prime}$ has either a gadget or a robust basis $B$. If $M^{\prime}$ has a gadget, then (a) holds by Lemma 2.5.13. Assume that $M^{\prime}$ has a robust basis $B$. Now if $M^{\prime}$ has at least two $(N, B)$-robust elements outside of $\{x, y\}$, then (i) holds by Lemma 2.7.5. Assume that $M^{\prime}$ has at most one $(N, B)$-robust element outside of $\{x, y\}$. If $M^{\prime}$ has no $(N, B)$-robust elements outside of $\{x, y\}$, then (b)(i) holds by Lemma 2.7.6. Assume that $M^{\prime}$ has an $(N, B)$-robust element $z$ outside of $\{x, y\}$. Suppose that $z$ is not $(N, B)$-strong. Then, by our choice of basis $B$, no basis of $M^{\prime}$ has an $(N, B)$-strong element outside of $\{x, y\}$. Now $A_{x x^{\prime}} \neq 0$ for some $x^{\prime} \in B^{*}-\{z\}$ because $M^{\prime}$ is 3-connected, so a pivot on $A_{x x^{\prime}}$ is allowable. Let $B^{\prime}=B \triangle\left\{x, x^{\prime}\right\}$. Then $B^{\prime}$ is another robust basis for $M^{\prime}$, and so $\left\{x^{\prime}, y, z\right\}$ is also a triad of $M^{\prime}$. Then $M^{\prime}$ has a 4-point cosegment $\left\{x, x^{\prime}, y, z\right\}$ gadget, so (a) holds by Lemma 2.5.13,

We may therefore assume that $z$ is $(N, B)$-strong, and that $\{x, y, z\}$ is a triad of $M^{\prime}$. If $M^{\prime}$ is $N$-fragile, then (b)(i) holds. Assume that $M^{\prime}$ has an $N$-flexible element. Since $N$-flexible elements are $(N, B)$-robust, it follows that the only $N$-flexible elements of $M \backslash a, b$ are in $\{x, y, z\}$. Since $z$ is $(N, B)$-strong, it follows from Lemma 2.4 .5 that $\{a, x, y\}$ or $\{b, x, y\}$ is a triangle of $M$. Assume that $\{b, x, y\}$ is a triangle of $M$. Then either $\{b, x, y, z\}$ or $\{a, b, x, y, z\}$ is a cocircuit of $M$ that contains the triangle $\{b, x, y\}$, so (b)(ii) holds.

We handle a few special cases next before we prove the second main result.

Lemma 2.7.8. If $\{a, b\} \subseteq \operatorname{cl}_{M}(\{x, y\})$, then $|E(M)| \leq|E(N)|+5$.

Proof. Suppose that $\{a, b\} \subseteq \operatorname{cl}_{M}(\{x, y\})$ but that $|E(M)| \geq|E(N)|+6$. Then there is at least one element $p$ in $E(M)-\{a, b, x, y, z\}$ that is either $N$-deletable or $N$-contractible in $M^{\prime}$. Suppose that $p$ is $N$-deletable. Then $p \in B-\{x, y\}$ because $M^{\prime}$ has no $(N, B)$-robust elements outside of $S^{\prime}$. Now $A_{p a}=A_{p b}=0$ because $\{a, b\} \subseteq \mathrm{cl}_{M}(\{x, y\})$. Moreover, there is some $q \in B^{*}-\{a, b\}$ such that $A_{p q} \neq 0$ because $p$ is not a coloop of $M^{\prime}$. Then a pivot on $A_{p q}$ is allowable, and $B^{\prime}=$ $B \triangle\{p, q\}$ is a basis for $M^{\prime}$ with more $\left(N, B^{\prime}\right)$-robust elements; a contradiction of 
the choice of $B$. We may therefore assume that $p$ is $N$-contractible in $M^{\prime}$. Suppose that $p \in \operatorname{cl}_{M}(\{x, y\})$. Then $p \in \operatorname{cl}_{M^{\prime}}\left(E\left(M^{\prime}\right)-S^{\prime}\right)$, so $\operatorname{co}\left(M^{\prime} \backslash z\right)$ is 3-connected by Bixby's lemma. But then $p$ is also $N$-deletable, so $p$ is an $(N, B)$-strong element outside of $S^{\prime}$; a contradiction. Thus $p \notin \operatorname{cl}(\{x, y\})$, so $A_{r p} \neq 0$ for some $r \in B-$ $\{x, y\}$. Moreover $A_{r a}=A_{r b}=0$ because $\{a, b\} \subseteq \operatorname{cl}_{M}(\{x, y\})$, so a pivot on $A_{r p}$ is allowable. But then $B^{\prime}=B \triangle\{r, p\}$ is a basis for $M^{\prime}$ with more $\left(N, B^{\prime}\right)$-robust elements; a contradiction of the choice of $B$.

Lemma 2.7.9. If $n=1$, and there is some $p \in B-\{x, y\}$ such that $p \in$ $\operatorname{cl}_{M}(\{x, y, z\})$ and $\{a, b\} \subseteq \operatorname{cl}_{M}(\{p, x, y\})$, then $r(M) \leq r(N)+4$ or $|E(M)| \leq$ $|E(N)|+7$.

Proof. Suppose that $\{x, y, z\}$ are the $(N, B)$-robust elements of $M$, and that $p \in$ $B-\{x, y\}$ is an element such that $p \in \operatorname{cl}_{M}(\{x, y, z\})$ and $\{a, b\} \subseteq \operatorname{cl}_{M}(\{p, x, y\})$. Then $A_{q a}=A_{q b}=0$ for all $q \in B-\{p, x, y\}$. Suppose that $|E(M)| \geq|E(N)|+8$ and $r(M) \geq r(N)+5$. Let $N=M^{\prime} / C \backslash D$. Then $C \cup D$ has at least two elements $q, q^{\prime}$ outside of $\{p, x, y, z\}$. Moreover, we can assume that $q$ is $N$-contractible in $M^{\prime}$ because $r(M) \geq r(N)+5$. Now if $q^{\prime}$ is $N$-deletable, then $q^{\prime} \in B$ because $q^{\prime}$ is not $(N, B)$-robust. For some $s \in B^{*}-\{a, b, z\}$ we have $A_{q^{\prime} s} \neq 0$ because $q^{\prime}$ is not a coloop of $M^{\prime}$, and $A_{q^{\prime} a}=A_{q^{\prime} b}=0$, so a pivot on $A_{q^{\prime} s}$ is allowable. But now $M^{\prime}$ has more $\left(N, B^{\prime}\right)$-robust elements for $B^{\prime}=B \triangle\left\{q^{\prime}, s\right\}$; a contradiction because $B$ is a robust basis. Thus $q^{\prime}$ is $N$-contractible. Then $q^{\prime} \in B^{*}-\{a, b, z\}$ because $q^{\prime}$ is not $(N, B)$-robust. Now if $q$ and $q^{\prime}$ are in $\operatorname{cl}_{M}(\{p, x, y\})$, then $q, q^{\prime} \in \mathrm{cl}_{M}(\{x, y, z\})$. Now $\left\{p, q, q^{\prime}\right\}$ is a triangle of $M^{\prime}$, and $q^{\prime}$ is $N$-contractible, so $q$ is also $N$-deletable because $\{p, q\}$ is a parallel pair of $M^{\prime} \backslash q^{\prime}$; a contradiction because $q$ is not $(N, B)$ robust. Thus we can assume that $q \notin \operatorname{cl}_{M}(\{p, x, y\})$. Then $A_{s q} \neq 0$ for some $s \in$ $B-\{p, x, y\}$, and $A_{s a}=A_{s b}=0$, so a pivot on $A_{s q}$ is allowable. But now $M^{\prime}$ has more $\left(N, B^{\prime}\right)$-robust elements for $B^{\prime}=B \triangle\{s, q\}$; a contradiction because $B$ is a robust basis.

We now prove that, up to duality and $\Delta-\nabla$-equivalence, we can find an excluded minor and a deletion pair that avoids the outcome of Theorem 2.7.7(b)(ii).

Theorem 2.7.10. Let $M$ be an excluded minor for the class of $\mathbb{P}$-representable matroids, and let $N$ be a strong $\mathbb{P}$-stabilizer. If $M$ has a pair of elements $a, b$ such that $M \backslash a, b$ is 3-connected with an $N$-minor, then there is some excluded minor $M^{\prime}$ that is $\Delta$-D-equivalent to $M^{\prime \prime} \in\left\{M, M^{*}\right\}$ with a minor $N^{\prime} \in\left\{N, N^{*}\right\}$, a pair of 
elements $a^{\prime}, b^{\prime}$ such that $M^{\prime} \backslash a^{\prime}, b^{\prime}$ is 3-connected with an $N^{\prime}$-minor, and a basis $B^{\prime}$ for $M^{\prime}$ such that at least one of the following holds:

(i) $\left|E\left(M^{\prime}\right)\right| \leq\left|E\left(N^{\prime}\right)\right|+16$; or

(ii) $\min \left\{r\left(M^{\prime}\right), r^{*}\left(M^{\prime}\right)\right\} \leq \max \left\{r\left(N^{\prime}\right), r^{*}\left(N^{\prime}\right)\right\}+7$; or

(iii) there is some robust basis $B^{\prime}$ for $M^{\prime}$ such that $M^{\prime} \backslash a^{\prime}, b^{\prime}$ is $N^{\prime}$-fragile, and $M^{\prime} \backslash a^{\prime}, b^{\prime}$ has at most one $\left(N^{\prime}, B^{\prime}\right)$-robust element $z \in\left(B^{\prime}\right)^{*}-\left\{a^{\prime}, b^{\prime}\right\}$ outside of $\left\{x^{\prime}, y^{\prime}\right\}$. Moreover, if $z \in\left(B^{\prime}\right)^{*}-\left\{a^{\prime}, b^{\prime}\right\}$ is an $\left(N^{\prime}, B^{\prime}\right)$-robust element, then $z$ is an $\left(N^{\prime}, B^{\prime}\right)$-strong element of $M^{\prime} \backslash a^{\prime}, b^{\prime}$, and $\left\{x^{\prime}, y^{\prime}, z\right\}$ is a triad of $M^{\prime} \backslash a^{\prime}, b^{\prime}$.

Proof. We may assume that Theorem 2.7.7(b)(ii) holds for $M$ and the pair $a, b$. Then $M \backslash a, b$ has exactly one $(N, B)$-robust element $z$ outside of $\{x, y\}$ and $\{x, y, z\}$ is a triad.

Then $M \backslash a, b$ has an $(N, B)$-strong element $z \in B^{*}-\{a, b\}$, and the only $(N, B)$ robust elements of $M^{\prime}$ are contained in the triad $\{x, y, z\}$. Note that $\{x, y\}$ is the only series pair for $M^{\prime} \backslash z$, for if $\{p, q\}$ were another series pair, then $\{p, q\}$ are $N$-contractible in $M^{\prime}$, and at least one of $p, q$ is in $B-\{x, y\}$, so $M^{\prime}$ has an $(N, B)$-robust element outside of $\{x, y, z\}$; a contradiction. We can assume that $b \in \operatorname{cl}_{M \backslash a, z}(\{x, y\})$ and $b \in \operatorname{cl}_{M \backslash a, z}^{*}(\{x, y\})$ by Lemma 2.4.5. Let $T$ be the triangle $\{b, x, y\}$ of $M$.

Now, at least one element of $\{x, y, z\}$ is $N$-flexible. Then, as $S^{\prime}$ is a triad and $z$ is $N$-deletable, the element $z$ must be $N$-flexible. We assume that neither (i) nor (ii) holds and prove that (iii) must hold.

We may assume that $a$ is not in the span of $\{b, x, y\}$ or (i) holds by Lemma 2.7.8. Next we show that we can find another excluded minor with a delete pair that is in a triangle.

2.7.10.1. If $M$ has a 4-element cocircuit $\{b, x, y, z\}$, then (i) or (ii) holds. If $M$ has a cocircuit $\{a, b, x, y, z\}$, then, up to $\Delta-\nabla$ equivalence and duality, there is a deletion pair $\{p, q\}$ for $M$ such that $M \backslash p, q$ is 3-connected with an $N$-minor, and $p$ and $q$ are in a triangle of $M$.

Subproof. Assume that $\{a, b\}$ are not in a triangle of $M$. Then $M$ has no triangle of the form $\{p, x, y\}$ for all $p \in E(M)-\{b\}$. Such an element would be $N$-deletable 
in $M^{\prime}$ because $x$ is contractible and $\{p, y\}$ is a parallel pair of $M^{\prime} / x$. But $p \in B^{*}$ because $\{p, x, y\}$ is a circuit and $\{x, y\} \subseteq B$; a contardiciton because $M^{\prime}$ has no $(N, B)$-robust elements outside of $\{x, y, z\}$.

Assume that $M \backslash b$ has triangles $\{p, x, z\}$ and $\{q, y, z\}$ for some $p, q \in E(M)-$ $\{b, x, y, z\}$. Suppose that $a \notin\{p, q\}$. Then, since $z$ is $N$-contractible and $\{p, x\}$, $\{q, y\}$ are parallel pairs, the elements $p, q$ are $N$-deletable in $M^{\prime}$. Then $p, q \in$ $B-\{x, y\}$ because there are no $(N, B)$-robust elements outside of $\{x, y, z\}$; a contradiction because $\{p, q, x, y, z\}$ is a rank-3 set so the 4-element subset $\{p, q, x, y\}$ is dependent. Thus $a \in\{p, q\}$, and $M$ has triangles $\{a, x, z\}$ and $\{p, y, z\}$. But again $p \in B-\{x, y\}$, so then $\{a, b\} \subseteq \operatorname{cl}_{M}(\{p, x, y\})$. Thus (i) or (ii) holds by Lemma 2.7.9. We may therefore assume that $M \backslash b$ has no triangles containing $\{x, y\}$ or $\{x, z\}$.

Either $\{b, x, y, z\}$ is a 4-element cocircuit of $M$ or $\{a, b, x, y, z\}$ is a 5-element cocircuit of $M$.

Assume that $\{b, x, y, z\}$ is a 4-element cocircuit of $M$. Then $\Delta_{T}(M)$ has a 4-point cosegment $\{b, x, y, z\}$. Now $\Delta_{T}(M) / b \cong M \backslash b$, so $\Delta_{T}(M) / b, t \cong M \backslash b / t$ for any $t \in E(M)-\{b\}$. Assume that in $M \backslash b$ there are either no triangles of the form $\{p, x, z\}$ or no triangles of the form $\{q, y, z\}$ for $p, q \in E(M)-\{b, x, y, z\}$. Then it follows from the dual of Tutte's Triangle Lemma and the fact that $M$ has no triangle of the form $\{p, x, y\}$ that $M \backslash b / x$ or $M \backslash b / y$ is 3-connected. Moreover, both $M \backslash b / x$ and $M \backslash b / y$ have an $N$-minor, because $x$ and $y$ are $N$-contractible in $M^{\prime}$ and hence $N$-contractible in $M \backslash b$. Thus $\Delta_{T}(M)$ has an $N$-minor, and for some $t \in\{x, y\}$, there is a pair of elements $b, t$ such that $\Delta_{T}(M) / b, t$ is 3-connected with an $N$-minor. Consider $\nabla_{T}\left(M^{*}\right)$, the dual of $\Delta_{T}(M)$. It follows that $\nabla_{T}\left(M^{*}\right)$ is an excluded minor with a delete pair on a 4-point segment $\{b, x, y, z\}$. Thus (i) or (ii) holds by Lemma 2.7.5 and Lemma 2.7.8.

We may assume that $\{a, b, x, y, z\}$ is a 5-element cocircuit of $M$. Now either $a$ is in a triangle with $z$ and one of $x$ and $y$ or it is not. Suppose that $\{a, z, x\}$ is a triangle. We claim that $\{b, x\}$ is the desired deletion pair. Clearly $M \backslash b$ is 3-connected and has an $N$-minor. Now $z$ is $N$-contractable in $M^{\prime}$, so $z$ is also $N$-contractible in $M \backslash b$. Now $x$ and $a$ are in parallel in $M \backslash b / z$, so $M \backslash b, x / z$ has an $N$-minor. Thus $M \backslash x, b$ has an $N$-minor. We now show that $M \backslash x, b$ is 3-connected.

Suppose that $\operatorname{co}(M \backslash b, x)$ is not 3-connected. Then there is a vertical 3-separation $(P, x, Q)$ for $(M \backslash b)^{*}$. By orthogonality we can assume that $z \in P$ and $a \in Q$. But 
now $y \in P \cup Q$, so assume that $y \in P$. Then $(P \cup x \cup a, Q-a)$ is an exact 3separation of $M$, so $a \in \mathrm{cl}^{(*)}(Q-a)$. But $a \in \operatorname{cl}(P \cup x)$ because $\{a, x, z\}$ is a triangle of $M \backslash b$ and $a \in \mathrm{cl}^{*}(P \cup x)$ because $\{a, x, y, z\}$ is a cocircuit of $M \backslash b$; a contradiction of orthogonality. Thus $\operatorname{co}(M \backslash b, x)$ is 3-connected. Now if $M \backslash b, x$ is not 3-connected, then there is a triad $C$ of $M \backslash b$ that contains $x$. By orthogonality with the triangle $\{a, x, z\}$ the triad $C$ meets $\{a, z\}$. But $a \notin C$ because $M \backslash a, b$ is 3-connected. Thus $M \backslash b$ has a triad $C$ that meets $\{x, z\}$. But then $C$ is a triad of $M \backslash a, b$ too, so $M^{\prime}$ has a 4-point cosegment $C \cup\{x, y, z\}$; a contradiction because $M^{\prime}$ has no gadget. Thus $M \backslash b, x$ is 3 -connected with an $N$-minor, and $\{b, x\}$ is contained in a triangle of $M$.

Assume that neither $\{a, z, x\}$ nor $\{a, z, y\}$ a triangle of $M$. Consider $\Delta_{T}(M)$. We claim that $\{b, x\}$ is a contract pair for $\Delta_{T}(M)$. We see that $\Delta_{T}(M) / b \cong M \backslash b$, so $\Delta_{T}(M) / b$ is 3 -connected and has an $N$-minor. Now $\Delta_{T}(M) / b, x \cong M \backslash b / x$. Now $x$ is $N$-contractible in $M^{\prime}$, so $M \backslash b / x$ has an $N$-minor. Thus $\Delta_{T}(M) / b, x$ has an $N$ minor. Suppose that $M \backslash b$ has a triangle $T^{\prime}$ that contains $x$. Then by orthogonality with the cocircuit $\{a, x, y, z\}$, and the fact that $M \backslash b$ has no triangles containing $\{x, y\}$ or $\{x, z\}$, the triangle $T^{\prime}$ must have the form $\{x, a, q\}$ for some $q \in E(M)-$ $\{a, b, x, y, z\}$. But now $q$ is $N$-deletable because $x$ is $N$-contractible in $M \backslash b$ and $\{a, q\}$ is a parallel pair of $M \backslash b / x$. Thus $q \in B-\{x, y\}$ because $M^{\prime}$ has no $(N, B)$ robust elements outside of $\{x, y, z\}$. Thus $\{a, b\} \subseteq \mathrm{cl}_{M}(\{q, x, y\})$, and so (i) or (ii) holds by Lemma 2.7.9. We may therefore assume that $M \backslash b$ has no triangle containing $x$. Since $x$ is not in a triangle of $M \backslash b$, it follows that if $M \backslash b / x$ is not 3-connected, then there is a vertical 3-separation $(P, x, Q)$ of $M \backslash b$. But $M \backslash a, b, z / x$ is 3-connected, so we can assume that $Q=\{a, z, q\}$ for some $q \neq y$. The set $Q$ is a triad of $M \backslash b$ since $Q$ is a 3-separating set and $r(Q) \geq 3$. But then $M \backslash a, b$ is not 3-connected because $a \in Q$ and $Q$ is a triad of $M \backslash b$; a contradiction. Thus $M \backslash b / x$, and hence $\Delta_{T}(M) / b, x$ is 3-connected with an $N$-minor. Since $\{b, x\}$ is contained in a triad of $\Delta_{T}(M)$, the dual $\nabla_{T}\left(M^{*}\right)$ has a delete pair $\{b, x\}$ that is contained in a triangle of $\nabla_{T}\left(M^{*}\right)$.

Up to replacing $M$ by a $\Delta$ - $\nabla$-equivalent matroid or its dual and replacing $\{a, b\}$ by another deletion pair, we can assume that $\{a, b\}$ is contained in a triangle of $M$. Suppose that the theorem fails for $M$ and $\{a, b\}$. Then by 2.7.10.1 there is a 5-element cocircuit $C=\{a, b, x, y, z\}$ of $M$. Since $a, b$ are contained in a triangle, either $\{a, b, z\}$ is a triangle of $M$ or $\{a, b, p\}$ is a triangle for some $p \notin\{x, y, z\}$. 
Call the first a type-I bad-cocircuit and the other a type-II bad-cocircuit.

Let $C^{+}=C$ if $M$ has a type-I bad-cocircuit and let $C^{+}=C \cup\{p\}$ if $M$ has a type-II bad-cocircuit.

2.7.10.2. There is an element $q$ of $M$ such that $M \backslash b, q$ is 3-connected with an $N$ minor, where $q$ is an element of $M$ such that $q \notin \mathrm{cl}_{M}^{*}\left(C^{+}\right)$and $q$ is not in a triangle of the form $\{q, x, z\}$ or $\{q, y, z\}$.

Subproof. We claim that there is some element $q$ of $M$ outside of $\{x, y, z\}$ such that $q \notin \mathrm{cl}_{M}^{*}\left(C^{+}\right)$and $q$ is not in a $\{q, x, z\}$ or $\{q, y, z\}$ triangle. We can assume that $r^{*}(M) \geq r^{*}(N)+8$ or (ii) holds for $M$ and $\{a, b\}$, so there are at least three $N$-deletable elements outside of $\operatorname{cl}_{M}^{*}\left(C^{+}\right)$. At most two of these elements can be in a triangle with $\{x, z\}$ or $\{y, z\}$ because $z$ is not on a 4-point segment of $M \backslash a, b$ by Lemma 2.4.2. Thus such a $q$ exists. Since $M \backslash a, b$ has no $(N, B)$-robust elements outside of $\{x, y, z\}$, it follows that $q \in B-\{x, y\}$. We show that there is an element $q$ with these properties such that $M \backslash b, q$ is 3 -connected with an $N$-minor.

Suppose that $\operatorname{co}(M \backslash a, b, q)$ is not 3-connected. Then there is a vertical 3separation $(X, q, Y)$ of $(M \backslash a, b)^{*}$ such that $|X \cap E(N)| \leq 1$ and $Y \cup q$ is closed in $(M \backslash a, b)^{*}$. But then it follows from Lemma 2.2.12 that at most one element of $X$ is not $N$-flexible, so at most one element of $X$ is not $(N, B)$-robust. We can assume that either $X=\{x, y, z\}$ or $X=\{p, x, y, z\}$ for some $p \in \mathrm{cl}_{M \backslash a, b}^{*}(X-\{p\})$ because the only $(N, B)$-robust elements of $M^{\prime}$ are in $\{x, y, z\}$. But then $r_{M \backslash a, b}^{*}(X)=2$ since $X$ is a triad of $M^{\prime}$; a contradiction because $(X, q, Y)$ is a vertical 3-separation of $(M \backslash a, b)^{*}$. Thus $\operatorname{co}(M \backslash a, b, q)$ is 3-connected.

Now suppose that $\{r, s\}$ is a series pair of $M \backslash a, b, q$, so $\{q, r, s\}$ is a triad of $M \backslash a, b$. Then $r$ and $s$ are $N$-contractible in $M \backslash a, b$. Now $z \notin\{r, s\}$ because $\{x, y\}$ is the only series pair of $M^{\prime} \backslash z$. Thus either $\{r, s\} \subseteq B^{*}-\{a, b, z\}$ or $\{r, s\}$ meets $\{x, y\}$. Suppose that $\{r, s\} \subseteq B^{*}-\{a, b, z\}$. If $\{q, r, s\}$ is a triad of $M$, then $A_{q a}=A_{q b}=0$, so there is an allowable pivot on $A_{q r}$ or $A_{q s}$ that gives a basis for $M \backslash a, b$ with more robust elements; a contradiction because $B$ is a robust basis. Now if $\{b, q, r, s\}$ or $\{a, b, q, r, s\}$ is a cocircuit of $M$, then we contradict orthogonality with the triangle $\{b, x, y\}$. Thus $M \backslash a, b, q$ is 3 -connected up to series pairs. Now suppose that $\{r, s\}$ meets $\{x, y\}$. If $\{r, s\}=\{x, y\}$, then $M \backslash a, b$ has a 4-point cosegment, so the size of $M$ is bounded by the gadget case. Thus we may assume that $r=x$ and that $s \neq y$. Now if $\{b, q, r, s\}$ is a cocircuit of $M$, then $s \in C^{+}$by orthogonality with the 
triangle containing $\{a, b\}$. But then $q \in \mathrm{cl}_{M}^{*}\left(C^{+}\right)$; a contradiction of the choice of $q$. Thus $\{b, q, r, s\}$ is not a cocircuit of $M$.

Now by the choice of $q$ we see that $M \backslash b, q$ has an $N$-minor, and that $M \backslash b$ is 3connected. Suppose that $\operatorname{co}(M \backslash b, q)$ is 3-connected. Then $M \backslash b, q$ is 3-connected because $M$ has no 4-element cocircuit of the form $\{b, q, r, s\}$. Suppose that $\operatorname{co}(M \backslash b, q)$ is not 3-connected. Now $\operatorname{co}(M \backslash a, b, q)$ is 3-connected, and $M$ has no 4-element cocircuit of the form $\{b, q, r, s\}$, so it follows that there is a vertical 3-separation $(P, q, Q)$ of $(M \backslash b)^{*}$ where $Q \cup q$ is a 4-element cocircuit of $M \backslash b$ and $Q=\{a, r, s\}$ is a triangle of $M \backslash b$ such that $a \notin \mathrm{cl}_{M \backslash b}(P)$. Then we may assume that $s=x$ by orthogonality with the triangle $Q$ and the cocircuit $\{a, x, y, z\}$. Now consider the element $r \in Q$. Since $x$ is contractible in $M \backslash b$ and $\{r, a\}$ is a parallel pair of $M \backslash b / x$, it follows that $M \backslash b, r$ has an $N$-minor. Suppose that $r \in \mathrm{cl}_{M}^{*}\left(C^{+}\right)$. Then $Q \cup b \subseteq C^{+}$, so $q \in \mathrm{cl}_{M}^{*}\left(C^{+}\right)$; a contradiction of the choice of $Q$. Thus $r \notin \mathrm{cl}_{M}^{*}\left(C^{+}\right)$. We claim that $M$ has no triangle of the form $\{r, x, z\}$ or $\{r, y, z\}$. If $M$ has a triangle of the form $\{r, x, z\}$, then $\{a, r, x, z\}$ is a 4-segment of $M$; a contradiciton of the property that $\operatorname{co}(M \backslash a, b, q)$ is 3-connected. The fact that $M$ has no triangle of the form $\{r, y, z\}$ follows from orthogonality with the cocircuit $Q \cup q$.

We claim that $M \backslash b, r$ is 3-connected. Suppose that $(X, r, Y)$ is a vertical 3separation of $(M \backslash b)^{*}$. By orthogonality we may assume that $a \in X$ and $x \in Y$. We may also assume without loss of generality that $q \in Y$. Now $X-a$ and $X$ are exactly 3-separating in $M \backslash b$, so $a \in \mathrm{cl}^{(*)}(X-a)$. But $a \in \operatorname{cl}(Y \cup\{a, r\})$ and $a \in \mathrm{cl}^{*}(Y \cup\{a, r\})$; a contradiciton of orthogonality. Thus if $M \backslash b, r$ is not 3connected, then there is some triad $T^{\prime}$ of $M \backslash b$ such that $r \in T^{\prime}$. By orthogonality with the triangle $\{a, r, x\}$, the triad $T^{\prime}$ meets $\{a, x\}$. But $a \notin T^{\prime}$ because $M \backslash a, b$ is 3-connected. Thus $r, x \in T^{\prime}$. Now either $T^{\prime}$ or $T^{\prime} \cup b$ is a cocircuit of $M$, and $\left|T^{\prime}-C^{+}\right| \geq 2$ because $r \notin \operatorname{cl}_{M}^{*}\left(C^{+}\right)$. But then $\{b, x, y\}$ and, for some $s \in C^{+}$, $\{a, b, s\}$ are triangles of $M$ that meet $T^{\prime}$ and $T^{\prime} \cup b$ in a single element, respectively; a contradiction of orthogonality.

Suppose that $B^{\prime}$ is a robust basis for $M \backslash b, q$, and let $A^{\prime}$ be the companion matrix for $M$ with $\left\{b, q, x^{\prime}, y^{\prime}\right\}$ an incriminating set for $\left(M, A^{\prime}\right)$. Finally, we show that there is no 5-element cocircuit of $M$ of the form $C^{\prime}=\left\{x^{\prime}, y^{\prime}, z^{\prime}, b, q\right\}$, where $\left\{x^{\prime}, y,{ }^{\prime} z\right\}$ is a triad of $M \backslash b, q$ with $(N, B)$-strong $z^{\prime}$ outside of $\left\{x^{\prime}, y^{\prime}\right\}$. Thus the (i) or (ii) holds for $M$ or (iii) holds for and $M \backslash b, q$.

Seeking a contradiction, suppose that $M$ has such a cocircuit $C^{\prime}=\left\{x^{\prime}, y^{\prime}, z^{\prime}, b, q\right\}$. 
Then $\left\{x^{\prime}, y^{\prime}\right\}$ is an unstable series pair for $z^{\prime}$, so either $b \in \operatorname{cl}_{M}\left(\left\{x^{\prime}, y^{\prime}\right\}\right)$ or $q \in$ $\operatorname{cl}_{M}\left(\left\{x^{\prime}, y^{\prime}\right\}\right)$.

Suppose that $b \in \operatorname{cl}_{M}\left(\left\{x^{\prime}, y^{\prime}\right\}\right)$. Since $M$ has a bad-cocircuit $C$, and triangles $\{b, x, y\}$ and $\{a, b, s\}$ for $s \in\{p, z\}$, it follows from orthogonality and the fact that $q \notin C$ that $\{x, y\}$ and $\{a, s\}$ meet $\left\{x^{\prime}, y^{\prime}, z^{\prime}\right\}$. Either $\{x, y\}=\left\{x^{\prime}, y^{\prime}\right\}$ or $\{x, y\}=\{a, s\}$ or there is another element in $\operatorname{cl}_{M}\left(\left\{x^{\prime}, y^{\prime}\right\}\right)$ that is not $b$; a contradiction of $z^{\prime}$ being $(N, B)$-strong in $M \backslash b, q$. Suppose that $\{x, y\}=\left\{x^{\prime}, y^{\prime}\right\}$. Then $z^{\prime} \in\{a, s\}$. If $a=z^{\prime}$, then $\{x, y, q\}$ is a triad of $M \backslash a, b$, so $\{q, x, y, z\}$ is a 4-point segment; a contradiction. Thus $s=z^{\prime}$. But then $C^{\prime}-\{q\} \subseteq C^{+}$so $q \in \mathrm{cl}_{M}^{*}\left(C^{+}\right)$; contradiction of the choice of $q$. Now suppose that $\{a, s\}=\left\{x^{\prime}, y^{\prime}\right\}$. Then $z^{\prime} \in\{x, y\}$. But again we have $C^{\prime}-\{q\} \subseteq C^{+}$, so $q \in \mathrm{cl}_{M}^{*}\left(C^{+}\right)$; a contradiction of the choice of $q$.

Now suppose that $q \in \operatorname{cl}_{M}\left(\left\{x^{\prime}, y^{\prime}\right\}\right)$. Both of the triangles $\{b, x, y\}$ and $\{a, b, s\}$ through $b$ meet $\left\{x^{\prime}, y^{\prime}, z^{\prime}\right\}$ by orthogonality with $C^{\prime}$. Suppose that $C$ is a typeII bad-cocircuit such that $p \in\left\{x^{\prime}, y^{\prime}\right\}$ and $z^{\prime} \in\{x, y\}$. Then $a$ is a basis element since $a$ is $N$-deletable in $M \backslash b, q$ and $a$ is not $\left(N, B^{\prime}\right)$-robust in $M \backslash b, q$. But then the $\{a, b, p\}$ triangle imples that $A_{b v}^{\prime}=0$ for $v \in\left\{x^{\prime}, y^{\prime}\right\}-\{p\}$; a contradiction because the entries of the bad submatrix are non-zero. Therefore the triangles through $b$ cannot meet $\left\{x^{\prime}, y^{\prime}\right\}$ in a single element by orthogonality with the bad-cocircuit $C$ and the triangle $\left\{x^{\prime}, y^{\prime}, q\right\}$. Thus $\left\{x^{\prime}, y^{\prime}\right\}$ is contained in $C$. But then we can assume $q$ is on a triangle of the form $\{x, a\}$ or $\{x, z\}$. If $q$ is on a segment of the form $\{x, z\}$, then we contradict the choice of $q$. If $q$ is on a triangle of the form $\{x, a\}$, then since $q, x \in B$ we have $A_{y a}=0$; a contradiction because the entries of the bad submatrix are non-zero. 


\section{Chapter 3}

\section{The structure of $\left\{U_{2,5}, U_{3,5}\right\}$-fragile matroids}

Let $M$ be a large excluded minor for the class of matroids representable over $\mathbb{H}_{5}$ or $\mathbb{U}_{2}$ partial field. Then it follows from the excluded-minor characterisation of the class of near-regular matroids that $M$ has a $\left\{U_{2,5}, U_{3,5}\right\}$-minor. Thus, by Theorem 2.1.2 of Chapter 2, if $M$ has a pair of elements $a, b$ such that $M \backslash a, b$ is 3-connected with a $\left\{U_{2,5}, U_{3,5}\right\}$-minor, then we can assume that $M \backslash a, b$ is a $U_{2,5^{-}}$or $U_{3,5}$-fragile matroid. It is then natural to ask: what is the structure of the $U_{2,5^{-}}$or $U_{3,5}$-fragile matroids representable over these partial fields?

An important subclass of the classes of $U_{2,5}$-fragile matroids and $U_{3,5}$-fragile matroids is the class of $\left\{U_{2,5}, U_{3,5}\right\}$-fragile matroids. In this chapter, we give a constructive description of the $\left\{U_{2,5}, U_{3,5}\right\}$-fragile matroids representable over these partial fields. Roughly speaking, these matroids fall into two classes. The matroids without an $\left\{X_{8}, Y_{8}, Y_{8}^{*}\right\}$-minor are constructed, up to duality, from one of two matroids by gluing wheels onto specified triangles. On the other hand, those matroids with an $\left\{X_{8}, Y_{8}, Y_{8}^{*}\right\}$-minor can be constructed from a matroid in $\left\{X_{8}, Y_{8}, Y_{8}^{*}\right\}$ by repeated application of elementary operations. These operations are, up to duality, either a parallel extension followed by a 4-element generalized $\Delta$ - $\nabla$-exchange or gluing a wheel onto a specified triangles. 


\subsection{Introduction}

Let $\mathscr{N}$ be a set of matroids. We denote the set of matroids $\left\{N^{*}: N \in \mathscr{N}\right\}$ by $\mathscr{N}^{*}$. We say that a matroid $M$ has an $\mathscr{N}$-minor if there is some minor $N$ of $M$ such that $N$ is isomorphic to a member of $\mathscr{N}$. Let $M$ be a matroid, and let $x$ be an element of $M$. If $M \backslash x$ has an $\mathscr{N}$-minor, then $x$ is $\mathscr{N}$-deletable. If $M / x$ has an $\mathscr{N}$-minor, then $x$ is $\mathscr{N}$-contractible. If neither $M \backslash x$ nor $M / x$ has an $\mathscr{N}$-minor, then $x$ is $\mathscr{N}$-essential. If $x$ is both $\mathscr{N}$-deletable and $\mathscr{N}$-contractible, then we say that $x$ is $\mathscr{N}$-flexible. We say that the matroid $M$ is $\mathscr{N}$-fragile if no element of $M$ is $\mathscr{N}$-flexible. If $M$ is $\mathscr{N}$-fragile and has an $\mathscr{N}$-minor, then we say that $M$ is strictly $\mathscr{N}$-fragile. In this chapter, whenever we omit the " $\mathscr{N}$-" prefix from these terms, assume that $\mathscr{N}=\left\{U_{2,5}, U_{3,5}\right\}$.

In [2] we apply the main result of [3] together with a case analysis to prove the following Theorem.

Theorem 3.1.1. [2. Theorem 1.3] Let $M^{\prime}$ be a 3-connected strictly $\left\{U_{2,5}, U_{3,5}\right\}$ fragile $\mathbb{H}_{5}$-representable matroid. Then $M^{\prime}$ is isomorphic to a matroid $M$ for which one of the following holds:

(i) $M$ has an $\left\{X_{8}, Y_{8}, Y_{8}^{*}\right\}$-minor;

(ii) $M \in\left\{U_{2,6}, U_{4,6}, P_{6}, M_{9,9}, M_{9,9}^{*}\right\}$;

(iii) $M$ or $M^{*}$ can be obtained from $U_{2,5}$ (with groundset $\{a, b, c, d, e\}$ ) by gluing wheels to $(a, c, b),(a, d, b),(a, e, b)$;

(iv) $M$ or $M^{*}$ can be obtained from $U_{2,5}$ (with groundset $\{a, b, c, d, e\}$ ) by gluing wheels to $(a, b, c),(c, d, e)$;

(v) $M$ or $M^{*}$ can be obtained from $M_{7,1}$ by gluing a wheel to $(1,3,2)$.

We also obtain the following Corollary of Theorem 3.1 .1

Corollary 3.1.2. [2] Corollary 1.4] Let $M^{\prime}$ be a 3-connected strictly $\left\{U_{2,5}, U_{3,5}\right\}$ fragile $\mathbb{U}_{2}$-representable matroid. Then $M^{\prime}$ is isomorphic to a matroid $M$ for which one of the following holds:

(i) $M$ has an $\left\{X_{8}, Y_{8}, Y_{8}^{*}\right\}$-minor;

(ii) $M \in\left\{M_{9,9}, M_{9,9}^{*}\right\}$; 
(iii) $M$ or $M^{*}$ can be obtained from $U_{2,5}$ (with groundset $\{a, b, c, d, e\}$ ) by gluing wheels to $(a, c, b),(a, d, b),(a, e, b)$;

(iv) $M$ or $M^{*}$ can be obtained from $U_{2,5}$ (with groundset $\{a, b, c, d, e\}$ ) by gluing wheels to $(a, b, c),(c, d, e)$;

(v) $M$ or $M^{*}$ can be obtained from $M_{7,1}$ by gluing a wheel to $(1,3,2)$.

In [2], the authors found all 3 -connected strictly $\left\{U_{2,5}, U_{3,5}\right\}$-fragile $\mathbb{H}_{5}$ representable matroids that have at most 9 elements. Figures $3.1-3.6$ give some geometric representations of these matroids. In the case of dual pairs, we have usually drawn only one of the two. Note that $M_{9,3}, M_{9,4}, M_{9,6}$, and their duals all have an $\left\{X_{8}, Y_{8}, Y_{8}^{*}\right\}$-minor. In Figure 3.5 we included a labeled version of $M_{7,1}$ and two of the rank-4 matroids containing it as a minor, $M_{8,6}$ and $M_{9,7}$, to illustrate the construction of matroids that have the property described in Theorem 3.1.1(v).

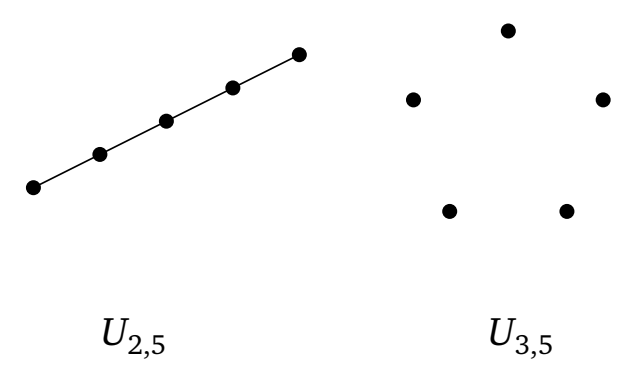

Figure 3.1: The 3-connected $\mathbb{H}_{5}$-representable fragile matroids on 5 elements.

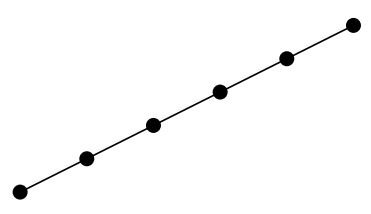

$U_{2,6}$

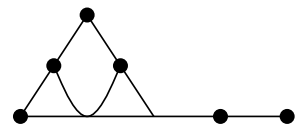

$Q_{6}$

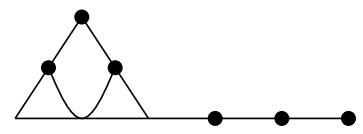

$P_{6}$

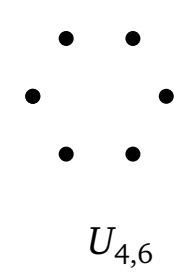

$U_{4,6}$

Figure 3.2: The 3-connected $\mathbb{H}_{5}$-representable fragile matroids on 6 elements.

A precise description of the operation of gluing a wheel to a triangle will be given later. However, to give the reader intuition for the operation, we highlight some of the geometric representations of matroids in Figures $3.3,3.6$ that are obtained by gluing wheels to triangles. The rank-4 matroids $M_{7,2}, M_{8,1}$ alternate, and $M_{9,0}$ are obtained from $U_{2,5}$ (with groundset $\{a, b, c, d, e\}$ ) by gluing 


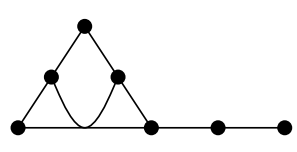

$M_{7,0}$

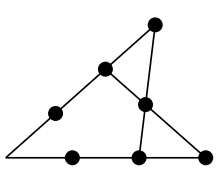

$M_{7,1}$

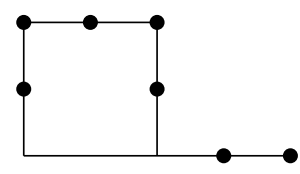

$M_{7,2}=M_{7,0}^{*}$

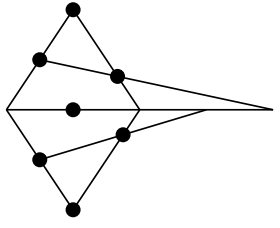

$M_{7,3}=M_{7,1}^{*}$

Figure 3.3: The 3-connected $\mathbb{H}_{5}$-representable fragile matroids on 7 elements.
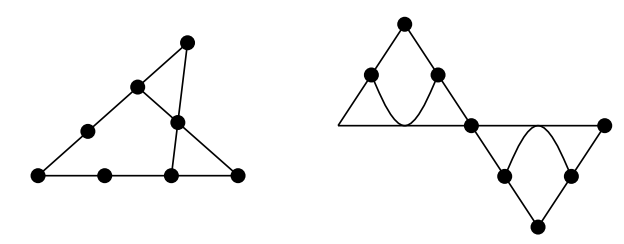

$Y_{8}=M_{8,0}=M_{8,7}^{*}$

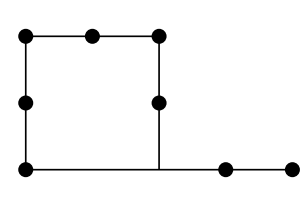

$M_{8,1}$, alternative

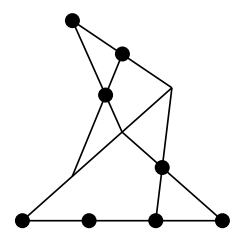

$X_{8}=M_{8,2}$

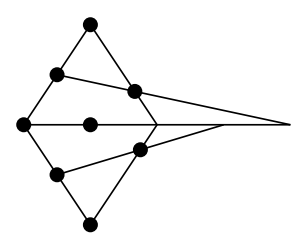

$M_{8,3}=M_{8,4}^{*}$

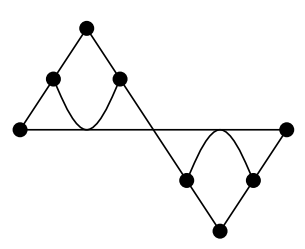

$M_{8,5}$

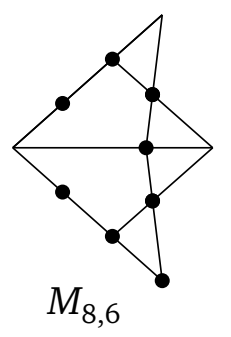

Figure 3.4: The 3-connected $\mathbb{H}_{5}$-representable fragile matroids on 8 elements.
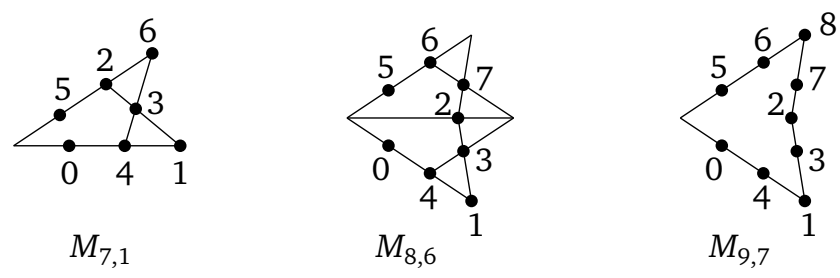

Figure 3.5: The matroids $M_{7,1}, M_{8,6}$, and $M_{9,7}$. In the right-most diagram, the 2-point lines were omitted to emphasize the fan $(1,3,2,7,8)$. 

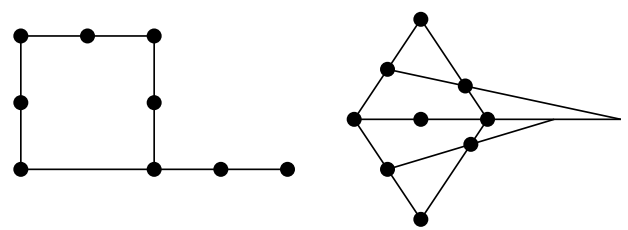

$$
M_{9,0}=M_{9,10}^{*}
$$

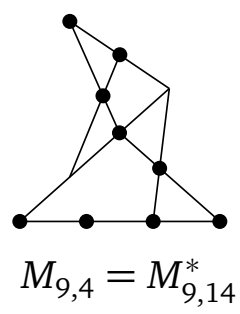

$$
M_{9,4}=M_{9,14}^{*}
$$

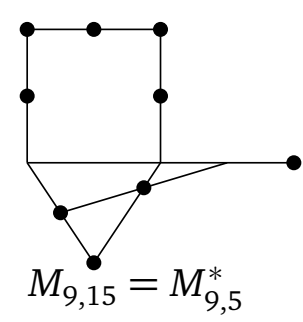

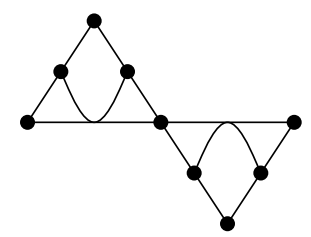

$M_{9,2}=M_{9,12}^{*}$

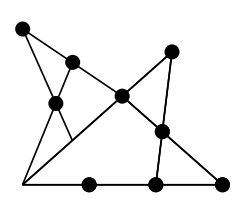

$M_{9,6}=M_{9,16}^{*}$
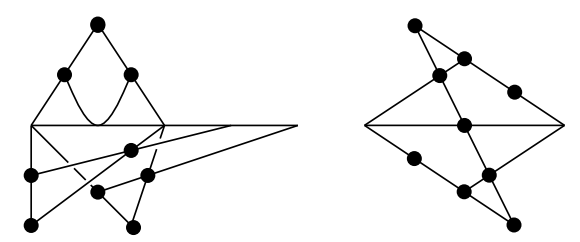

$$
M_{9,18}=M_{9,8}^{*} \quad M_{9,9}=M_{9,19}^{*}
$$

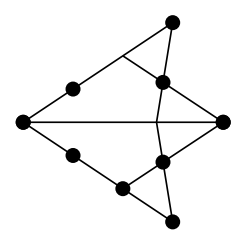

$$
M_{9,3}=M_{9,13}^{*}
$$

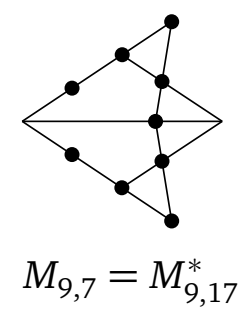
, 17

Figure 3.6: The 3-connected $\mathbb{H}_{5}$-representable fragile matroids on 9 elements. 
a rank-4 wheel to a triangle; the rank-4 matroids $M_{7,3}, M_{8,3}$, and $M_{9,1}$ are obtained from $U_{2,5}$ (with groundset $\{a, b, c, d, e\}$ ) by gluing rank-3 wheels to the triangles $(a, c, b)$ and $(a, d, b)$; the rank-4 matroids $M_{8,1}, M_{8,5}$, and $M_{9,2}$ are obtained from $U_{2,5}$ (with groundset $\{a, b, c, d, e\}$ ) by gluing rank-3 wheels to the triangles $(a, b, c)$ and $(c, d, e)$; the rank-5 matroid $M_{9,15}$ is obtained from $U_{2,5}$ (with groundset $\{a, b, c, d, e\})$ by gluing a rank-4 wheel to the triangle $(a, c, b)$, and then gluing a rank-3 wheel to the triangle $(a, d, b)$; the rank-5 matroid $M_{9,18}$ is obtained from $U_{2,5}$ (with groundset $\{a, b, c, d, e\}$ ) by gluing rank-3 wheels to the triangles $(a, c, b),(a, d, b)$, and $(a, e, b)$.

In this Chapter, we augment Threorem 3.1.1 and Corollary 3.1.2 by giving a constructive description of the 3 -connected $\mathbb{U}_{2}$ - and $\mathbb{H}_{5}$-representable strictly $\left\{U_{2,5}, U_{3,5}\right\}$-fragile matroids that have an $\left\{X_{8}, Y_{8}, Y_{8}^{*}\right\}$-minor. Roughly speaking, in addition to growing fans as in the other classes, we can also build paths of 3 -separations along the 4-element segments and cosegments of the matroids in $\left\{X_{8}, Y_{8}, Y_{8}^{*}\right\}$. In order to describe the structure of these matroids more formally we need some definitions.

Let $M$ be a matroid with an $\mathscr{N}$-minor. A subset $S$ of $E(M)$ is a segment if every 3-element subset of $S$ is a triangle. We say a segment $S$ of a matroid $M$ is $\mathscr{N}$ allowable if $S$ is coindependent and some element of $S$ is not $\mathscr{N}$-deletable. A subset $C$ is a cosegment if $C$ is a segment of $M^{*}$. A cosegment of $M$ is $\mathscr{N}$ allowable if it is an $\mathscr{N}^{*}$-allowable segment of $M^{*}$. We say $S$ is an $\mathscr{N}$-allowable set if $S$ is either an $\mathscr{N}$-allowable segment or an $\mathscr{N}$-allowable cosegment of $M$.

Let $A$ be a set in a matroid $M$. Recall that $A$ is fully closed if $A$ is closed in both $M^{*}$ and $M$. The full closure of $A$, denoted $\operatorname{fcl}_{M}(A)$ is the intersection of all fully closed sets containing $A$. One easy way of obtaining the full closure of a set $A$ is to take $\mathrm{cl}_{M}(A)$ and then $\mathrm{cl}_{M}^{*}\left(\mathrm{cl}_{M}(A)\right)$ and continue this way until no new elements are added via closure or coclosure. We call $A$ a path-generating set if $A$ is a 3separating set of $M$ such that $\mathrm{fcl}_{M}(A)=E(M)$. Note that $A$ is a path-generating set if and only if the complement of $A$ is a sequential 3-separating set. A pathgenerating set $A$ thus gives rise to a natural path of 3 -separations $\left(P_{1}, \ldots, P_{m}\right)$, where the end step $P_{1}$ is $A$ and each step $P_{i}$ is either the closure or coclosure of the 3-separation $P_{1} \cup \cdots \cup P_{i-1}$. The path-generating sets in this chapter will be allowable sets or fans.

Let $C$ be an $\mathscr{N}$-allowable cosegment $M$. A matroid $Q$ is an $\mathscr{N}$-allowable series 
extension along $C$ if $M=Q / S$ and, for every element $s$ of $S$ there is some element $c$ of $C$ such that $c$ is $\mathscr{N}$-contractible in $M$ and $s$ is in series with $c$ in $Q$. We also say that $Q^{*}$ is an $\mathscr{N}^{*}$-allowable parallel extension along $C$.

The generalized $\Delta-\nabla$-exchange was introduced by Oxley, Semple, and Vertigan [21], and we will repeat their definition in Section 3.2. Intuitively speaking, the generalized $\Delta-\nabla$-exchange gives a new matroid by exchanging a segment and a cosegment in an way that is analogous to the exchange of a triad and a triangle in the familiar $\Delta-\nabla$-exchange. Let $M$ and $N$ be matroids. We say that $M$ is obtained from $N$ by a $\Delta-\nabla$-step along $A$ if, up to duality, $M$ is obtained from $N$ by performing an $\mathscr{N}$-allowable parallel extension along a path-generating allowable set $A$ of $N$, followed by a generalized $\Delta-\nabla$-exchange on $A$.

Let $N$ be a matroid, and let $T$ be an $\mathscr{N}$-allowable triangle of $M$ with non-deletable element $x \in T$. Let $\mathscr{W}_{r}$ be the rank-r wheel, with some triangle labelled by $T$ such that $x$ labels the rim element. We let $P_{T}\left(M\left(\mathscr{W}_{r}\right), N\right)$ denote the generalized parallel connection of $M\left(\mathscr{W}_{r}\right)$ and $N$ along $T$ (see Oxley [20]). Following the terminology from [3], we say that $M$ is obtained from $N$ by gluing a wheel to the triangle $T$ if $M=P_{T}\left(M\left(\mathscr{W}_{r}\right), N\right) \backslash X$ for some integer $r$ and subset $X$ of $T$ such that $x \in T$. We also say that $M^{*}$ is obtained from $N^{*}$ by gluing a wheel to the triad $T$. It follows from the definition of the generalized parallel connection that $E\left(M\left(\mathscr{W}_{r}\right)\right)-X$ is a fan of $M$. Note that when $r=3$ and $X=T$, the operation of gluing a wheel to $T$ corresponds to performing a $\Delta$ - $\nabla$-exchange on $T$.

A sequence of matroids $M_{1}, \ldots, M_{n}$ is called a path-sequence if the following conditions hold:

(a) $M_{1}=X_{8}$; and

(b) For each $i \in\{1, \ldots, n-1\}$, there is some 4-element path-generating segment or cosegment $A$ of $M_{i}$ such that either:

(i) $M_{i+1}$ is obtained from $M_{i}$ by a $\Delta-\nabla$-step along $A$; or

(ii) $M_{i+1}$ is obtained from $M_{i}$ by gluing a wheel along an allowable subset $A^{\prime}$ of $A$.

A path sequence $M_{1}, \ldots, M_{n}$ obtains a matroid $M$ if $M_{n} \cong M$. Note that since $X_{8}$ is self dual it is easy to see that a $M_{1}^{*}, \ldots, M_{n}^{*}$ is also a path sequence. We can now state the main result of the chapter. 
Theorem 3.1.3. If $M$ is a 3-connected $\left\{U_{2,5}, U_{3,5}\right\}$-fragile $\mathbb{H}_{5}$-representable matroid that has an $\left\{X_{8}, Y_{8}, Y_{8}^{*}\right\}$-minor, then there is some path sequence that obtains $M$.

We also have the following Corollary for the class of $\mathbb{U}_{2}$-representable matroids.

Corollary 3.1.4. If $M$ is a 3-connected $\left\{U_{2,5}, U_{3,5}\right\}$-fragile $\mathbb{U}_{2}$-representable matroid that has an $\left\{X_{8}, Y_{8}, Y_{8}^{*}\right\}$-minor, then there is some path sequence that obtains $M$.

In Figure 3.4, we observe that there is an allowable triad $T$ of $X_{8}$ such that $Y_{8}$ is isomorphic to $\nabla_{T}\left(X_{8}\right)$, so there are path sequences that obtain $Y_{8}$ and $Y_{8}^{*}$ respectively. Moreover, we observe that the rank-4 matroids $M_{9,3}, M_{9,4}$, and $M_{9,6}$ of Figure 3.6 can be obtained from $Y_{8}$ by gluing wheels to allowable triangles, so there are also path sequences that obtain these matroids and their duals.

Looking at Theorem 3.1 .3 and Corollary 3.1 .4 with a view towards an excludedminor characterisation of the classes of $\mathbb{H}_{5^{-}}$and $\mathbb{U}_{2}$-representable matroids, it seems that the most important knowledge we gain about the structure of a matroid $M$ obtained by a path sequence is that $M$ has a path of 3 -separations $\left(P_{1}, \ldots, P_{m}\right)$, where the end step $P_{1}$ is either a 4-element allowable set or a fan, while each internal step $P_{i}$ has at most three elements and $P_{i}$ is either the closure or coclosure of the 3-separating set $P_{1} \cup \cdots \cup P_{i-1}$.

The Chapter is organised as follows. Section 3.2 contains essential background results on connectivity, fragility, and the generalized $\Delta-\nabla$-exchange. We then define the class of fragile matroids that arise from path sequences, and then establish some properties about path sequence rearrangement. We then establish the structure of our proof of Theorem 3.1 .3 by identifying three cases for a minimum-sized counterexample. We either eliminate or establish a bound on the size of a counterexample for each case in the following three sections. Finally, we conclude the proof of Theorem 3.1 .3 with a case analysis of the fragile matroids.

\subsection{Preliminaries}

If $M$ is a connected matroid such that $\min \{r(X), r(Y)\}=1$ or $\min \left\{r^{*}(X), r^{*}(Y)\right\}=1$ for every 2-separation $(X, Y)$ of $M$, then we say 
that $M$ is 3-connected up to series and parallel classes. The next result implies that every $\left\{U_{2,5}, U_{3,5}\right\}$-fragile matroid is 3 -connected up to series and parallel classes.

Proposition 3.2.1. [19. Proposition 4.3] Let $M$ be a matroid with a 2-separation $(A, B)$, and let $N$ be a 3-connected minor of $M$. Assume $|E(N) \cap A| \geq|E(N) \cap B|$. Then $|E(N) \cap B| \leq 1$. Moreover, unless $B$ is a parallel or series class, there is an element $x \in B$ such that both $M \backslash x$ and $M / x$ have a minor isomorphic to $N$.

The following is an easy consequence of the property that strictly $\left\{U_{2,5}, U_{3,5}\right\}$ fragile matroids are 3-connected up to parallel and series classes. It enables us to do $\Delta$ - $\nabla$-exchanges on any appropriately labelled segments and cosegments.

Lemma 3.2.2. Let $M$ be a strictly $\left\{U_{2,5}, U_{3,5}\right\}$-fragile matroid with at least 8 elements. If $S$ is a triangle or 4-segment of $M$ such that $E(M)-S$ is not a series or parallel class of $M$, then $S$ is coindependent in $M$. If $C$ is a triad or 4-cosegment of $M$ such that $E(M)-S$ is not a series or parallel class of $M$, then $C$ is independent.

The generalized $\Delta-\nabla$-exchange of Oxley, Semple, and Vertigan [21] is used frequently. Let $M$ be a matroid with a $U_{2, k}$-restriction $A$, and suppose $A$ is coindependent in $M$. The generalized $\Delta-\nabla$-exchange on $A$, denoted by $\Delta_{A}(M)$, is defined to be the matroid $P_{A}\left(\Theta_{k}, M\right) \backslash A$ (see Section 1.1 for the definition of $\Theta_{k}$ ). Let $M$ be a matroid such that $M^{*}$ has a $U_{2, k}$-restriction $A$, and suppose $A$ is independent in $M$. The generalized $\Delta$ - $\nabla$-exchange on $A$, denoted by $\nabla_{A}(M)$, is defined to be the matroid $\left(P_{A}\left(\Theta_{k}, M^{*}\right) \backslash A\right)^{*}$. That is, $\nabla_{A}(M)=\left(\Delta_{A}\left(M^{*}\right)\right)^{*}$.

We now state some of the key properties of the $\Delta$ - $\nabla$-exchange here.

Lemma 3.2.3. [21] Lemma 2.5] For all $k \geq 2$, the restriction of $\left(P_{A}\left(\Theta_{k}, M\right) \backslash A\right)^{*}$ to $E\left(\Theta_{k}\right)-A$ is isomorphic to $U_{2, k}$ if and only if $A$ is coindependent in $M$.

Lemma 3.2.4. [21] Lemma 2.11] Let $A$ be a coindependent segment of a matroid M. Then $\nabla_{A}\left(\Delta_{A}(M)\right)$ is well-defined and $\nabla_{A}\left(\Delta_{A}(M)\right)=M$.

A key property of the $\Delta-\nabla$-exchange is that it preserves $\mathbb{P}$-representability for any partial field $\mathbb{P}$.

Lemma 3.2.5. [21. Lemma 3.4] $\Theta_{3}$ is regular, and $\Theta_{4}$ is near-regular. 
Lemma 3.2.6. [21. Lemma 3.5] Let $k \geq 2$, and let $M$ be a matroid such that $M \mid A \cong$ $U_{2, k}$. Let $\mathbb{P}$ be a partial field. If $M$ and $\Theta_{k}$ are $\mathbb{P}$-representable, then the generalized parallel connection $P\left(\Theta_{k}, M\right)$ of $\Theta_{k}$ and $M$ across $A$ is $\mathbb{P}$-representable.

Corollary 3.2.7. [21] Lemma 3.7] Let $\mathbb{P}$ be a partial field. Then $M$ is $\mathbb{P}$ representable if and only if $\Delta_{A}(M)$ is $\mathbb{P}$-representable.

The following two results on minors are crucial for removing elements from a matroid with a path sequence.

Lemma 3.2.8. [21] Lemma 2.13] Suppose that $\Delta_{A}(M)$ is defined. If $x \in A$ and $|A| \geq 3$, then $\Delta_{A-x}(M \backslash x)$ is also defined, and $\Delta_{A}(M) / x=\Delta_{A-x}(M \backslash x)$.

Lemma 3.2.9. [21] Lemma 2.16] Suppose that $\Delta_{A}(M)$ is defined.

(i) If $x \in E(M)-A$ and $A$ is coindependent in $M \backslash x$, then $\Delta_{A}(M \backslash x)$ is defined and $\Delta_{A}(M) \backslash x=\Delta_{A}(M \backslash x)$;

(ii) If $x \in E(M)-\operatorname{cl}(A)$, then $\Delta_{A}(M / x)$ is defined and $\Delta_{A}(M) / x=\Delta_{A}(M / x)$;

The next two results are employed for rearranging path sequences.

Lemma 3.2.10. [21] Lemma 2.18] Let $M$ be a matroid, and $S$ and $T$ be disjoint subsets of $E(M)$ such that $|S| \geq 2$ and $|T| \geq 2$. If $S$ and $T$ are both coindependent segments, then

$$
\Delta_{S}\left(\Delta_{T}(M)\right)=\Delta_{T}\left(\Delta_{S}(M)\right)
$$

Corollary 3.2.11. [21. Corollary 2.19] Let $M$ be a matroid, and $S$ and $T$ be disjoint subsets of $E(M)$ such that $|S| \geq 2$ and $|T| \geq 2$.

(i) If $S$ and $T$ are both independent cosegments, then

$$
\nabla_{S}\left(\nabla_{T}(M)\right)=\nabla_{T}\left(\nabla_{S}(M)\right)
$$

(ii) If $S$ is an independent cosegment and $T$ is a coindependent segment, then

$$
\nabla_{S}\left(\Delta_{T}(M)\right)=\Delta_{T}\left(\nabla_{S}(M)\right)
$$

The next two results give conditions for allowable segments and cosegments to go up the minor order, and are used later in reductions. 
Lemma 3.2.12. Let $M, M \backslash x$ be strictly $\mathscr{N}$-fragile matroids such that $M \backslash x$ has no $\mathscr{N}$-essential elements. If $A \subseteq E(M \backslash x)$ is an $\mathscr{N}$-allowable segment of $M \backslash x$, then $A$ is an $\mathscr{N}$-allowable segment of $M$.

Proof. Clearly $A$ is a coindependent segment of $M$. Suppose that $y \in A$ is not $\mathscr{N}$-deletable in $M \backslash x$. Then $y$ is contractible in $M \backslash x$ because $M \backslash x$ has no essential elements. But then $y$ is also contractible in $M$, and $M$ is $\mathscr{N}$-fragile, so $y$ is not $\mathscr{N}$-deletable in $M$.

Lemma 3.2.13. Let $M, M \backslash x$ be strictly $\mathscr{N}$-fragile matroids such that $M \backslash x$ has no essential elements. If $A \subseteq E(M \backslash x)$ is an $\mathscr{N}$-allowable cosegment of $M \backslash x$ and $x \in \operatorname{cl}(E(M)-(A \cup x))$, then $A$ is an $\mathscr{N}$-allowable cosegment of $M$.

Proof. Assume that $A \subseteq E(M \backslash x)$ is an $\mathscr{N}$-allowable cosegment of $M \backslash x$ and $x \in \operatorname{cl}(E(M)-(A \cup x))$. Then $A$ is a coindependent segment of $M^{*} / x$, and $x \notin \mathrm{cl}^{*}(A)$, so $A$ is a coindependent segment of $M^{*}$. Finally, since $M \backslash x$ has no essential elements $A$ has a deletable element in $M \backslash x$ and hence in $M$.

We have the following properties on connectivity, rank and closure for the $\Delta-\nabla$ exchange.

Lemma 3.2.14. [13] Lemma 9.3] Let $M$ be a 3-connected matroid.

(i) If $A$ is a segment of $M$, then, for all $A^{\prime} \subseteq A, P_{A}\left(\Theta_{k}, M\right) \backslash A^{\prime}$ is 3-connected up to series pairs.

(ii) If $A$ is a cosegment of $M$, then, for all $A^{\prime} \subseteq A,\left(P_{A}\left(\Theta_{k}, M\right) \backslash A^{\prime}\right)^{*}$ is 3connected up to parallel pairs.

Lemma 3.2.15. [13] Lemma 9.1] Let $M$ be a matroid, and let $X$ be a subset of $E(M)$.

(a) If $A$ is an allowable segment of $M$, then

(i) $r_{\Delta_{A}(M)}(X)=r_{M}(X)+|A|-2$ if $A \subseteq X$; and

(ii) $r_{\Delta_{A}(M)}(X)=r_{M}(X)$ if $A$ is disjoint from $X$.

(b) If $A$ is an allowable cosegment of $M$, then

(i) $r_{\nabla_{A}(M)}(X)=r_{M}(X)-|A|+2$ if $A \subseteq X$; and 
(ii) $r_{\nabla_{A}(M)}(X)=r_{M}(X)$ if A is disjoint from $X$.

The following lemma, whose proof is an elementary application of Lemma 3.2.15. shows that performing $\Delta-\nabla$-exchanges on a set $A$ of a matroid preserves the connectivity of separations that are nested with $A$.

Lemma 3.2.16. Let $M$ be a matroid, and let $(X, Y)$ be a partition of $E(M)$. If $A$ is an allowable segment of $M$, and $A \subseteq X$, then $\lambda_{M}(X)=\lambda_{\Delta_{A}(M)}(X)$. Moreover, for any $e \notin A$ and $Z \in\{X, Y\}, e \in \mathrm{cl}_{M}(Z)$ if and only if $e \in \mathrm{cl}_{\Delta_{A}(M)}(Z)$ and $e \in \mathrm{cl}_{M}^{*}(Z)$ if and only if $e \in \mathrm{cl}_{\Delta_{A}(M)}^{*}(Z)$.

By duality and Lemma 3.2.16, we have the following result.

Corollary 3.2.17. Let $M$ be a matroid, and let $(X, Y)$ be a partition of $E(M)$. If $A$ is an allowable cosegment of $M$, and $A \subseteq X$, then $\lambda_{M}(X)=\lambda_{\nabla_{A}(M)}(X)$. Moreover, for any $e \notin A$ and $Z \in\{X, Y\}, e \in \operatorname{cl}_{M}(Z)$ if and only if $e \in \operatorname{cl}_{\nabla_{A}(M)}(Z)$ and $e \in$ $\mathrm{cl}_{M}^{*}(Z)$ if and only if $e \in \mathrm{cl}_{\nabla_{A}(M)}^{*}(Z)$.

We now work towards a proof that, under certain conditions, the $\Delta$ - $\nabla$-exchange preserves fragility. We use the excluded-minor characterisation of near-regular matroids, but we note there is possibly a more general proof that avoids using such a deep result.

Theorem 3.2.18. [12] Theorem 1.2] The excluded minors for the class of nearregular matroids are $U_{2,5}, U_{3,5}, F_{7}, F_{7}^{*}, F_{7}^{-},\left(F_{7}^{-}\right)^{*}, \operatorname{AG}(2,3) \backslash e,(\operatorname{AG}(2,3) \backslash e)^{*}$, $\Delta_{T}(\mathrm{AG}(2,3) \backslash e)$, and $P_{8}$.

By combining partial-field homomorphism results of [35, Lemma 2.5.24] and [32, Corollary 3.1.3] we have the following.

Lemma 3.2.19. Let $M$ be $\mathbb{P}$-representable for some $\mathbb{P} \in\left\{\mathbb{U}_{2}, \mathbb{H}_{5}\right\}$. If $\mathbb{F}$ is a field with at least 5 elements, then $M$ is $\mathbb{F}$-representable.

We let $\mathscr{E} \mathscr{X}\left(\mathbb{U}_{1}\right)$ denote the set of excluded minors for the class of near-regular matroids. We now obtain a representability certificate of losing the $\left\{U_{2,5}, U_{3,5}\right\}$ minor.

Lemma 3.2.20. Let $M$ be a $\mathbb{U}_{2}$ - or $\mathbb{H}_{5}$-representable matroid. Then $M$ is nearregular if and only if $M$ has no $\left\{U_{2,5}, U_{3,5}\right\}$-minor. 
Proof. If $M$ is near-regular, then $M$ has no $\left\{U_{2,5}, U_{3,5}\right\}$-minor by Theorem 3.2 .18 . Conversely, suppose $M$ is $\mathbb{P}$-representable for some $\mathbb{P} \in\left\{\mathbb{U}_{2}, \mathbb{H}_{5}\right\}$ and that $M$ has no $\left\{U_{2,5}, U_{3,5}\right\}$-minor. It follows from Theorem 3.2 .18 and well-known results (see [12, 20]) that, for each matroid $M^{\prime}$ in $\mathscr{E} \mathscr{X}\left(\mathbb{U}_{1}\right)-\left\{U_{2,5}, U_{3,5}\right\}$, there is some prime power $q \geq 5$ such that $M^{\prime}$ is not $\mathrm{GF}(q)$-representable. Then $M$ also has no minor in $\mathscr{E} \mathscr{X}\left(\mathbb{U}_{1}\right)-\left\{U_{2,5}, U_{3,5}\right\}$ by Lemma 3.2 .19 , so $M$ is near-regular.

We can now show how the $\Delta-\nabla$-operations can be used to build new fragile matroids.

Lemma 3.2.21. Let $\mathbb{P} \in\left\{\mathbb{U}_{2}, \mathbb{H}_{5}\right\}$, and let $M$ be a strictly $\left\{U_{2,5}, U_{3,5}\right\}$-fragile $\mathbb{P}$-representable matroid. If $A$ is an allowable triangle of $M$, then $\Delta_{A}(M)$ is a $\left\{U_{2,5}, U_{3,5}\right\}$-fragile $\mathbb{P}$-representable matroid. Moreover, $A$ is an allowable triad of $\Delta_{A}(M)$.

Proof. It follows immediately from Lemma 3.2.7 and Lemma 3.2.20 that $\Delta_{A}(M)$ is $\mathbb{P}$-representable matroid with a $\left\{U_{2,5}, U_{3,5}\right\}$-minor. Moreover, $A$ is an independent triad of $\Delta_{A}(M)$ by Lemma 3.2.3. It remains to show that $\Delta_{A}(M)$ is $\left\{U_{2,5}, U_{3,5}\right\}$-fragile, and that some element of $A$ is non-contractible in $\Delta_{A}(M)$.

Since $A$ is an allowable triangle, there is some $x \in A$ that is non-deletable in $M$. Then $M \backslash x$ is near-regular by Lemma 3.2 .20 , so $\Delta_{A-x}(M \backslash x)$ is also near-regular by Lemma 3.2.7. But $\Delta_{A}(M) / x=\Delta_{A-x}(M \backslash x)$ by Lemma 3.2.8, so $\Delta_{A}(M) / x$ has no $\left\{U_{2,5}, U_{3,5}\right\}$-minor by Lemma 3.2 .20 . Thus $x$ is non-contractible in $\Delta_{A}(M)$, so $A$ is an allowable triad of $\Delta_{A}(M)$. Now suppose that $\Delta_{A}(M) \backslash y$ has a $\left\{U_{2,5}, U_{3,5}\right\}$-minor for some $y \in A-x$. But $A-y$ is a non-trivial series class of $\Delta_{A}(M) \backslash y$, so $x$ is contractible in $\Delta_{A}(M) \backslash y$; a contradiction because $x$ is non-contractible in $\Delta_{A}(M)$. Thus each $y \in A-x$ is non-deletable in $\Delta_{A}(M)$.

Suppose that $e \in E(M)-A$ is non-deletable in $M$. Then $M \backslash e$ is near-regular by Lemma 3.2.20. Now $A$ is a coindependent triangle in $M \backslash e$ and $\Delta_{A}(M \backslash e)$ is near-regular by Lemma 3.2.7. Thus $\Delta_{A}(M \backslash e)$ has no $\left\{U_{2,5}, U_{3,5}\right\}$-minor. But $\Delta_{A}(M \backslash e) \cong \Delta_{A}(M) \backslash e$, so $e$ is non-deletable in $\Delta_{A}(M)$.

Now suppose that $e \in E(M)-A$ is deletable but non-contractible in $M$, so $|E(M)| \geq 6$. Suppose that $e \in \mathrm{cl}_{M}(A)-A$. Then $(A, E(M)-A)$ is a 3-separation of $\Delta_{A}(M)$ and $e$ is in the guts of $(A, E(M)-A)$. Seeking a contradiction, suppose that $e$ is contractible $\Delta_{A}(M)$. Then $\Delta_{A}(M) / e$ has a non-minimal 2-separation $(A, E(M)-(A \cup e))$, and so $|E(N) \cap A| \leq 1$. Now $A$ is a triangle of $\Delta_{A}(M) / e$, so 
in particular, each element $x \in A$ is deletable in $\Delta_{A}(M) / e$ and hence in $\Delta_{A}(M)$; a contradiction because there is some non-deletable element of $\Delta_{A}(M)$ in $A$. Thus $e \in \mathrm{cl}_{M}(A)-A$ is non-contractible in $\Delta_{A}(M)$. We may therefore assume that $e \notin \mathrm{cl}_{M}(A)-A$. Then $A$ is a coindependent triangle of $M / e$ and $\Delta_{A}(M / e)$ is welldefined. Now $\Delta_{A}(M) / e=\Delta_{A}(M / e)$ by Lemma 3.2.9. But $M / e$ is near-regular, so $\Delta_{A}(M / e)$ is near-regular. Therefore $\Delta_{A}(M / e)$ has no $\left\{U_{2,5}, U_{3,5}\right\}$-minor by Lemma 3.2.20, and so $e$ is non-contractible in $\Delta_{A}(M)$.

We can extend Lemma 3.2 .21 by replacing the $\Delta-\nabla$-exchange with the 4-element generalized $\Delta$ - $\nabla$-exchange in the following lemma.

Lemma 3.2.22. Let $\mathbb{P} \in\left\{\mathbb{U}_{2}, \mathbb{H}_{5}\right\}$, and let $M$ be a strictly $\left\{U_{2,5}, U_{3,5}\right\}$-fragile $\mathbb{P}$ representable matroid. Let $A$ be an allowable 4-segment of $M$ such that, if $M$ has an element e such that $M \mid(A \cup e) \cong U_{2,5}$, then $e$ is non-deletable in $M$. Then $\Delta_{A}(M)$ is a strictly $\left\{U_{2,5}, U_{3,5}\right\}$-fragile $\mathbb{P}$-representable matroid. Moreover, $A$ is an allowable 4-cosegment of $\Delta_{A}(M)$.

Proof. It follows immediately from Lemma 3.2.7 and Lemma 3.2.20 that $\Delta_{A}(M)$ is $\mathbb{P}$-representable matroid with a $\left\{U_{2,5}, U_{3,5}\right\}$-minor. Moreover, $A$ is an independent 4-cosegment of $\Delta_{A}(M)$ by Lemma 3.2.3. It remains to show that $\Delta_{A}(M)$ is $\left\{U_{2,5}, U_{3,5}\right\}$-fragile, and that some element of $A$ is non-contractible in $\Delta_{A}(M)$.

Since $A$ is an allowable 4-segment, there is some $x \in A$ that is non-deletable in $M$. Then $M \backslash x$ is near-regular by Lemma 3.2.20, so $\Delta_{A-x}(M \backslash x)$ is also near-regular by Lemma 3.2.7. But $\Delta_{A}(M) / x=\Delta_{A-x}(M \backslash x)$ by Lemma 3.2.8, so $\Delta_{A}(M) / x$ has no $\left\{U_{2,5}, U_{3,5}\right\}$-minor by Lemma 3.2.20. Thus $x$ is non-contractible in $\Delta_{A}(M)$, so $A$ is an allowable 4-cosegment of $\Delta_{A}(M)$. Now suppose that $\Delta_{A}(M) \backslash y$ has a $\left\{U_{2,5}, U_{3,5}\right\}$-minor for some $y \in A-x$. But $A-y$ is a non-trivial series class of $\Delta_{A}(M) \backslash y$, so $x$ is contractible in $\Delta_{A}(M) \backslash y$; a contradiction because $x$ is noncontractible in $\Delta_{A}(M)$. Thus each $y \in A-x$ is non-deletable in $\Delta_{A}(M)$.

Suppose that $e \in E(M)-A$ is non-deletable in $M$, but that $\Delta_{A}(M) \backslash e$ has a $\left\{U_{2,5}, U_{3,5}\right\}$-minor. Then $P_{M \mid A}\left(\Theta_{4}, M\right) \backslash e$ has a $\left\{U_{2,5}, U_{3,5}\right\}$-minor. But $\Theta_{4}$ and $M \backslash e$ are near-regular, and $P_{M \mid A}\left(\Theta_{4}, M\right) \backslash e=P_{M \mid A}\left(\Theta_{4}, M \backslash e\right)$ by [20, Proposition 11.4.14(vi)], so $P_{M \mid A}\left(\Theta_{4}, M \backslash e\right)$ is also near-regular by Lemma 3.2.6; a contradiction to Lemma 3.2.20. Therefore $e$ is non-deletable in $\Delta_{A}(M)$.

Assume $e \in E(M)-A$ and that $e$ is deletable, and hence non-contractible, in $M$. Suppose $e \in \operatorname{cl}_{M}(A)$. Then by hypothesis $e \in \operatorname{cl}_{M}(\{a\})$ for some $a \in A$. 
We claim that $e$ is non-contractible in $\Delta_{A}(M)$. Clearly $\Delta_{A}(M) / e$ is isomorphic to a minor of $P_{M \mid A}\left(\Theta_{4}, M\right) / a$. By [20, Proposition 11.4.14(vii)], we have $P_{M \mid A}\left(\Theta_{4}, M\right) / a=P_{(M \mid A) / a}\left(\Theta_{4} / a, M / a\right)$. But $\Theta_{4} / a$ and $M / a$ are near-regular, so $P_{(M \mid A) / a}\left(\Theta_{4} / a, M / a\right)$ is also near-regular by Lemma 3.2.6. Thus $\Delta_{A}(M) / e$ has no $\left\{U_{2,5}, U_{3,5}\right\}$-minor by Lemma 3.2.20, so $e$ is indeed non-contractible in $\Delta_{A}(M)$. We can therefore assume $e \notin \operatorname{cl}_{M}(A)$, so that $\Delta_{A}(M) / e=\Delta_{A}(M / e)$ by Lemma 3.2.9. But $M / e$ is near-regular, so $\Delta_{A}(M / e)$ is near-regular. Therefore $\Delta_{A}(M / e)$ has no $\left\{U_{2,5}, U_{3,5}\right\}$-minor by Lemma 3.2.20, and so $e$ is non-contractible in $\Delta_{A}(M)$.

There are two ways we view fans: gluing a wheel onto a triangle, or sequences of moves where, up to duality, each move is a parallel extension followed by a $\Delta-\nabla$ exchange. From the construction perspective it makes sense to use the notion of gluing a wheel onto a triangle, but later for doing reductions it helps to view the fan as coming from some sequence of $\Delta-\nabla$-exchanges.

Recall that, in our definition of gluing a wheel to a triangle, the non-deletable element of the allowable triangle must label the rim element of the corresponding triangle of the wheel, and that this element must be deleted after the wheel is glued to the original matroid. Thus if we want to view the operation of gluing a wheel to a triangle as a sequence of moves where, up to duality, each move is a parallel extension followed by a $\Delta-\nabla$-exchange parallel extension followed by a $\Delta$ - $\nabla$-exchange, then the parallel extension of each move cannot put an element in parallel with the non-deletable element of the allowable triangle.

Lemma 3.2.23. Let $\mathbb{P} \in\left\{\mathbb{U}_{2}, \mathbb{H}_{5}\right\}$, and let $M$ be a strictly $\left\{U_{2,5}, U_{3,5}\right\}$-fragile $\mathbb{P}$-representable matroid. Let $A$ be an allowable triangle of $M$ contained in a 4-segment of $M$. If the matroid $M^{\prime}$ obtained from $M$ by gluing a wheel onto $A$ is 3-connected, then $M^{\prime}$ is a strictly $\left\{U_{2,5}, U_{3,5}\right\}$-fragile $\mathbb{P}$-representable matroid. Moreover, if $F$ is the set of elements of the fan, then the spoke elements of $F$ are deletable in $M^{\prime}$ and the rim elements of $F$ are contractible in $M^{\prime}$.

Proof. We proceed by induction on the rank of the wheel glued onto $T=(a, b, c)$. The base case when $r=3$ follows immediately from Lemma 3.2.21. Assume that it holds for $r$, and let $M^{\prime}$ is obtained from $M$ by gluing a rank- $(r+1)$ wheel onto $T$. Suppose that the fan of $M^{\prime}$ has with rim end corresponding to the point $c$. Now let $M^{\prime \prime}$ be the matroid obtained from gluing a rank- $r$ wheel 
onto $T$. By the inductive hypothesis $M^{\prime \prime}$ is fragile. Let $\left(a_{1}, \ldots, a_{n}\right)$ be the corresponding fan of $M^{\prime \prime}$, where $a_{n}$ is a spoke element corresponding to $c$, and $A^{\prime}=\left\{a_{n-2}, a_{n-1}, a_{n}\right\}$ is an allowable triangle such that $a_{n-2}$ and $a_{n}$ are noncontractible and $a_{n-1}$ is non-deletable. Let $N$ be the allowable parallel extension of $M^{\prime \prime}$ along $A^{\prime}$ such that $N \backslash a_{n+1}=M$ and $a_{n+1}$ is in parallel with $a_{n-2}$. We claim that $\Delta_{A}(N)$ is a $\left\{U_{2,5}, U_{3,5}\right\}$-fragile $\mathbb{P}$-representable matroid that has an $(n+1)$ element fan $F=\left(a_{1}, \ldots, a_{n-2}, a_{n}, a_{n-1}, a_{n+1}\right)$ of rank- $r+1$ and that $M^{\prime} \cong \Delta_{A}(N)$ where $A=\left\{a_{n-1}, a_{n}, a_{n+1}\right\}$ is an allowable triad such that $a_{n}$ non-contractible while $a_{n}$ and $a_{n+1}$ are non-deletable.

That $M^{\prime}$ is a $\left\{U_{2,5}, U_{3,5}\right\}$-fragile $\mathbb{P}$-representable matroid and that $A$ is an allowable triad of $M^{\prime}$ such that $a_{n}$ non-contractible while $a_{n}$ and $a_{n+1}$ are non-deletable all follows from Lemma 3.2.21. It remains to show that $\left\{a_{n-2}, a_{n}, a_{n-1}\right\}$ is a triangle of $M^{\prime}$, and in the case that $n \geq 4$, we show that $\left\{a_{n-3}, a_{n-2}, a_{n}\right\}$ is a triad of $M^{\prime}$. It follows from $\Delta$ - $\nabla$ Lemmas that $\left\{a_{n-2}, a_{n}, a_{n-1}\right\}$ is a triangle of $M^{\prime}$. To see that $\left\{a_{n-3}, a_{n-2}, a_{n}\right\}$ is a triad of $M^{\prime}$, we observe that in the matroid $P_{T}\left(M\left(K_{4}\right), N\right)$ the complement of $\left\{a_{n-3}, a_{n-2}, a_{n-1}, a_{n+1}, a_{n-1}^{\prime}\right\}$ is a hyperplane, where $a_{n-1}^{\prime}$ is the element relabelled by $a_{n-1}$ in $M^{\prime}$. Thus $\left\{a_{n-3}, a_{n-2}, a_{n}\right\}$ is a triad in $M^{\prime}=P\left(M\left(K_{4}\right), N\right) \backslash A$, as required. Finally, that $M^{\prime} \cong \Delta_{A}(N)$ follows from [24, Theorem 1.8] and the fact that $\Delta_{A}(N)$ is 3-connected.

The argument in the case when the fan of $M^{\prime}$ has a spoke end corresponding to $c$ is almost identical. We instead take $N$ is the allowable parallel extension of $M^{\prime \prime}$ along $A^{\prime}$ such that $M=N \backslash\left\{a_{n+1}, a_{n+2}\right\}$ where $a_{n+1}$ is in parallel with $a_{n-2}$ and $a_{n+2}$ is in parallel with $a_{n}$. Then $\Delta_{A}(N)$ where $A=\left\{a_{n-1}, a_{n}, a_{n+1}\right\}$ is a $\left\{U_{2,5}, U_{3,5}\right\}$-fragile $\mathbb{P}$-representable matroid that has an $(n+2)$-element rank$r+1$ fan $F=\left(a_{1}, \ldots, a_{n-2}, a_{n}, a_{n-1}, a_{n+1}, a_{n+2}\right)$ where $A$ is an allowable triad, and $\left\{a_{n-1}, a_{n+1}, a_{n+2}\right\}$ is an allowable triangle.

\subsection{The class}

Let $A \subseteq E(M)$. We call $A$ a path-generating set if $A$ is an allowable set of $M$ such that $\mathrm{fcl}_{M}(A)=E(M)$. For example, the 4-segments of $Y_{8}$ are path-generating sets, but the triangles of $Y_{8}$ that meet both the 4-segments are not path-generating sets. It is easy to see that the notion of path-generating sets is invariant under duality, because both the notion of allowable sets and full closure are dual invariant. 
Let $M$ and $M^{\prime}$ be matroids, and let $A$ be a 4-element path-generating segment or cosegment of $M$. We use the notation $M^{\prime}=\Delta_{A}^{N}(M)$ and $M^{\prime}=\nabla_{A}^{N}(M)$ if there is some allowable parallel or series extension $N$ of $M$ along $A$ such that $M^{\prime}=\Delta_{A}(N)$ or $M^{\prime}=\nabla_{A}(N)$.

We restate the definition of path sequences here for convenience.

A sequence of matroids $M_{1}, \ldots, M_{n}$ is called a path sequence for $M_{n}$ if the following conditions hold:

(a) $M_{1}=X_{8}$; and

(b) For each $i \in\{1, \ldots, n-1\}$, there is some 4-element path-generating segment or cosegment $A$ of $M_{i}$ such that either:

(i) $M_{i+1}=\Delta_{A}^{N}\left(M_{i}\right)$ or $M^{\prime}=\nabla_{A}^{N}\left(M_{i}\right)$; or

(ii) $M_{i+1}$ is obtained from $M_{i}$ by gluing a fan along an allowable subset $A^{\prime}$ of $A$.

Let $\mathscr{P}$ denote the class of matroids such that $M \in \mathscr{P}$ if and only if, up to seriesparallel classes, there is some path sequence $M_{1}, \ldots, M_{n}$ with $M_{n}=M$. It is easy to see from the definition that $\mathscr{P}^{*}=\mathscr{P}$.

The following is an easy consequence of the definition of path sequences, together with Lemma 3.2.22 and Lemma 3.2.23.

Lemma 3.3.1. Let $\mathbb{P} \in\left\{\mathbb{U}_{2}, \mathbb{H}_{5}\right\}$. If $M$ is a 3-connected matroid obtained by a path sequence, then $M$ is a strictly $\left\{U_{2,5}, U_{3,5}\right\}$-fragile $\mathbb{P}$-representable matroid. Moreover, $M$ has a path of 3-separations $\boldsymbol{P}=\left(P_{1}, \ldots, P_{n}\right)$ such that the end steps $P_{1}$ and $P_{n}$ are path-generating sets, and, for each internal step $P_{i}$ of $\boldsymbol{P},\left|P_{i}\right| \leq 3$ and $P_{i}$ is alternately the guts and coguts of the 3-separation $\left(P_{1} \cup \cdots \cup P_{i}, P_{i+1} \cup \cdots \cup\right.$ $\left.P_{n}\right)$.

We call $M_{i}$ the $i$-th step of the path sequence $M_{1}, \ldots, M_{n}$. We also call the $n$-th step the end step. The path-generating set is said to be used by a step.

It follows from the definition of a path sequence that, when a path-generating maximal fan or 4-element segment or cosegment is chosen to be the end step $P_{1}$, the path of 3-separations of Lemma 3.3.1 is uniquely determined. Furthermore, each 3 -connected matroid in $\mathscr{P}$ has exactly two sets that could be chosen as the end step, and that these sets can meet in at most one element. 


\subsection{Path sequence rearrangement}

Path sequences are generally not unique. That is, we can have distinct path sequences $M_{1}, \ldots, M_{n}$ and $M_{1}^{\prime}, \ldots, M_{n}^{\prime}$ such that $M_{n} \cong M_{n}^{\prime}$. One reason for this is that $X_{8}$ has two disjoint path-generating sets, and the order in which these are used does not change the resulting matroid obtained by a path sequence. Another reason is due to the symmetry of $X_{8}$. We will focus on the ends of path sequences in the following sections, and we often speak of path sequences with a particular ordering of steps. In this section we show when we can obtain a path sequence with a desired ordering. The results found here are mostly routine and follow from corresponding properties of the $\Delta$ - $\nabla$-operation. In the following sections, we refer to the results of this section with "by rearrangement".

We call a path sequence a $\Delta$ - $\nabla$-sequence if only moves of type (b)(i) are used. That is, if no step involves gluing a wheel to an allowable set.

Let $M_{1}, \ldots, M_{n}$ be a $\Delta$ - $\nabla$-sequence, and let $S$ and $C$ be the 4-element segment and cosegment of $X_{8}$ respectively. There is an associated sequence $a\left[M_{1}, \ldots, M_{n}\right]:\{2, \ldots, n\} \rightarrow\{S, C\}$ for $M_{1}, \ldots, M_{n}$ where, for $i \in\{2, \ldots n\}$, we define $a\left[M_{1}, \ldots, M_{n}\right](i)=X$ if $M_{i}$ is obtained from $M_{i-1}$ by using the set $X$. We call $a\left[M_{1}, \ldots, M_{n}\right]$ the adjacency sequence for $M_{1}, \ldots, M_{n}$. The adjacency sequence records the order of the steps used by the path sequence. It will not appear in the following sections.

Moves on disjoint path-generating sets commute.

Lemma 3.4.1. Let $M_{1}, \ldots, M_{n}$ be a $\Delta$ - $\nabla$-sequence. If $a\left[M_{1}, \ldots, M_{n}\right](i)=C$ and $a\left[M_{1}, \ldots, M_{n}\right](i+1)=S$ for some $i \in\{2, \ldots, n\}$, then there is some $\Delta$ - $\nabla$-sequence $M_{1}^{\prime}, \ldots, M_{n}^{\prime}$ such that $M_{n}=M_{n}^{\prime}$, and $a\left[M_{1}, \ldots, M_{n}\right](j) \neq a\left[M_{1}^{\prime}, \ldots, M_{n}^{\prime}\right](j)$ if and only if $j \in\{i, i+1\}$.

Proof. Suppose that $a\left[M_{1}, \ldots, M_{n}\right](i)=C$ and $a\left[M_{1}, \ldots, M_{n}\right](i+1)=S$ for some $i \in\{2, \ldots, n\}$. Suppose that $M_{i}=\Delta_{C}^{N_{i}}\left(M_{i-1}\right)$ and $M_{i+1}=\Delta_{S}^{N_{i+1}}\left(M_{i}\right)$. Let $Q=E\left(N_{i}\right)-E\left(M_{i-1}\right)$ and $R=E\left(N_{i+1}\right)-E\left(M_{i}\right)$, and consider the matroid $P=$ $\nabla_{C}\left(\nabla_{S}\left(M_{i+1}\right)\right)$. Then $P$ is a parallel extension of $M_{i-1}$ with the elements of $Q$ along $C$ and the elements of $R$ along $S$. Now $P_{i}=P \backslash Q$ is an allowable parallel extension of $M_{i-1}$ along $S$, so $M_{i}^{\prime}=\Delta_{S}^{P_{i}}\left(M_{i-1}\right)$ is obtained from $M_{i-1}$ using $S$. Next $P_{i+1}=\Delta_{S}(P)$ is an allowable parallel extension of $M_{i}^{\prime}$ along $C$, so $M_{i+1}^{\prime}=\Delta_{C}^{P_{i+1}}\left(M_{i}^{\prime}\right)$ is obtained from $M_{i}^{\prime}$ using $C$. But now $M_{i+1}^{\prime}=\Delta_{C}\left(\Delta_{S}(P)\right)$, so 
$M_{i+1}^{\prime}=M_{i+1}$ by Lemma 3.2.10. The argument when $M_{i}$ or $M_{i+1}$ use the $\nabla$ operation is symmetric, with Corollary 3.2.11 used in place of Lemma 3.2.10.

By repeated application of Lemma 3.4.1 we can choose the set used by the end step.

Lemma 3.4.2. Let $M_{1}, \ldots, M_{n}$ be a $\Delta$ - $\nabla$-sequence. Let $A \in\{S, C\}$. If the adjacency sequence for $M_{1}, \ldots, M_{n}$ is non-constant, then there is a path-sequence $M_{1}^{\prime}, \ldots, M_{n}^{\prime}$ such that $M_{n} \cong M_{n}^{\prime}$ and $M_{n}^{\prime}$ is obtained from $M_{n-1}$ using A. Moreover, if the path-adjacency sequence for $M_{1}, \ldots, M_{n}$ takes the value $A$ at least $k$ times, then we can assume that the last $k$ steps of $M_{1}^{\prime}, \ldots, M_{n}^{\prime}$ use $A$.

We now look at some consequences of the symmetry of $X_{8}$.

Lemma 3.4.3. Let $M=\nabla_{C}^{N}\left(X_{8}\right)$ for some allowable series-extension $N$ of $X_{8}$ along $C$. Then, for any allowable parallel-extension $N^{\prime}$ of $M$ along $C$, there is some allowable parallel-extension $N^{\prime \prime}$ of $M$ along $S$ such that $\Delta_{C}^{N^{\prime}}(M) \cong \Delta_{S}^{N^{\prime \prime}}(M)$.

Proof. Suppose that $M=\nabla_{C}^{N}\left(X_{8}\right)$ for some allowable series-extension $N$ of $X_{8}$ along $C$. Now it is easy to see that $X_{8} \cong \nabla_{S}(Q)$ where $Q$ is an allowable series extension of $U_{3,5}$ such that $E(Q)=S \cup C$, so let $R$ be the allowable series extension of $Q$ with the elements of $N$ added along $C$. Then $N \cong \nabla_{S}(R)$. Thus $\Delta_{S}(M) \cong N$ by Lemma 3.2.11 and Lemma 3.2.4. But by Lemma 3.2.4 we also have $\Delta_{C}(M)=N$. Thus for any allowable parallel-extension $N^{\prime}$ of $M$ along $C$, there is some allowable parallel-extension $N^{\prime \prime}$ of $M$ along $S$ such that $N^{\prime} \cong N^{\prime \prime}$, and hence $\Delta_{C}^{N^{\prime}}(M) \cong \Delta_{S}^{N^{\prime \prime}}(M)$.

The next lemma shows that a $\Delta$ - $\nabla$-sequence can finish at either end of the associated path of 3-separations.

Lemma 3.4.4. Let $M_{1}, \ldots, M_{n}$ be a $\Delta$ - $\nabla$-sequence such that $a\left[M_{1}, \ldots, M_{n}\right](i)=C$ for all $i \in\{2, \ldots, n\}$. Then there is some equivalent sequence $M_{1}^{\prime}, \ldots, M_{n}^{\prime}$ such that $a\left[M_{1}^{\prime}, \ldots, M_{n}^{\prime}\right](n)=S$.

Proof. We prove by induction on the number of steps. The case $n=1$ follows from Lemma 3.4.3. Suppose for induction that it holds for $n-1$. Then there is some sequence $M_{1}^{\prime}, \ldots, M_{n-1}^{\prime}$ equivalent to $M_{1}, \ldots, M_{n-1}$ such that $a\left[M_{1}^{\prime}, \ldots, M_{n-1}^{\prime}\right](n-1)=S$. Then $M_{1}^{\prime}, \ldots, M_{n-1}^{\prime}, M_{n}$ is a sequence for $M_{n}$ that is 
non-constant, so by Lemma 3.4 .2 there is some equivalent sequence $M_{1}^{\prime \prime}, \ldots, M_{n}^{\prime \prime}$ such that $a\left[M_{1}^{\prime \prime}, \ldots, M_{n}^{\prime \prime}\right](n)=S$.

If a $\Delta$ - $\nabla$-sequence has at least two steps, then there are $\Delta$ - $\nabla$-sequences with consecutive steps using either $S$ or $C$.

Lemma 3.4.5. Let $M_{1}, \ldots, M_{n}$ be a $\Delta$ - $\nabla$-sequence such that the last $k$ steps use $X \in\{S, C\}$. If $M_{1}, \ldots, M_{n}$ has at least $k+1$ steps, then there is some equivalent $\Delta$ - $\nabla$-sequence $M_{1}^{\prime}, \ldots, M_{n}^{\prime}$ such that the last $k+1$ steps use $X$.

Proof. Suppose that $M_{1}, \ldots, M_{n}$ has at least $k+1$ steps. If there are $k+1$ steps using $X$, then the Lemma follows from Lemma 3.4.2. Assume that only the last $k$ steps use $X$. Since there are at least $k+1$ steps, the remaining steps must use $Y=X-(S \cup C)$. In particular, the first step must use $Y$. But then by Lemma 3.4.3 there is an equivalent $\Delta-\nabla$-sequence $M_{1}^{\prime}, \ldots, M_{n}^{\prime}$ such that the first step uses $X$. Now it follows from Lemma 3.4 .2 that there is an equivalent $\Delta-\nabla$-sequence $M_{1}^{\prime \prime}, \ldots, M_{n}^{\prime \prime}$ such that the last $k+1$ steps use $X$, as required.

We now return to considering general path sequences. Since gluing a wheel along an allowable triangle is the same as a sequence of allowable parallel-extensions followed by a $\Delta-\nabla$-exchange, it again follows easily from Lemma 3.2 .10 and Corollary 3.2 .11 that this operation commutes with moves on a disjoint pathgenerating set. Thus we have the following Lemma.

Lemma 3.4.6. Let $M_{1}, \ldots, M_{n}$ be a path sequence for $M$, and let $F$ be a fan of $M$. If, for some $i \in\{1, \ldots, n-1\} M_{i+1}$ is obtained from $M_{i}$ by gluing the fan $F$ along $A^{\prime} \subseteq A$, then there is a path sequence $M_{1}^{\prime}, \ldots, M_{n}^{\prime}$ for $M$ such that $M_{n}^{\prime}$ is obtained from $M_{n-1}^{\prime}$ by gluing a wheel along $A^{\prime} \subseteq A$. Moreover, if $M_{1}, \ldots, M_{n}$ has at least one $\Delta-\nabla$-move, then we can assume that $M_{n-1}^{\prime}$ is obtained from $M_{n-2}^{\prime}$ by a $\Delta$ - $\nabla$-move using $A$.

\subsection{The setup}

First we show that every matroid in the class $\mathscr{P}$ has a minor in $\mathscr{S}=$ $\left\{X_{8}, Y_{8}, Y_{8}^{*}, M_{8,6}\right\}$.

Lemma 3.5.1. Let $M$ be a matroid. If $M$ can be obtained by a path sequence, then $M$ has a minor in $\mathscr{S}=\left\{X_{8}, Y_{8}, Y_{8}^{*}, M_{8,6}\right\}$. 
Proof. Let $M_{1}, \ldots, M_{n}$ be a path sequence for $M$. Note that fan moves for $|F| \geq$ 4 can be removed by contracting all but one rim element, then simplifying any parallel classes.

If there are no $\Delta-\nabla$-steps, then $M$ has a minor in $\mathscr{S}$. If there is a single $\Delta$ - $\nabla$-step, then $M$ has a minor in $\mathscr{S}$. Thus assume that $M$ has at least two $\Delta-\nabla$-steps and that they are consecutive. We may assume for induction that any path sequence with fewer $\Delta$ - $\nabla$-steps than $M$ has an $\mathscr{S}$-minor.

Now, up to duality, we can assume that $M_{k}=\Delta_{A}^{N}\left(M_{k-1}\right)$ and that $M_{k-1}=$ $\nabla_{A}^{Q}\left(M_{k-2}\right)$ for $k \in\{n-1, n\}$. Let $N^{\prime}=E(N)-E\left(M_{k-1}\right)$. Now either $M=M_{k}$ or $M=\Delta_{A^{\prime}}\left(M_{k}\right)$ for some allowable triangle $A^{\prime} \subseteq A$. Thus $M \backslash N^{\prime}=M_{k} \backslash N^{\prime}$ or $M \backslash N^{\prime}=\Delta_{A^{\prime}}\left(M_{k} \backslash N^{\prime}\right)$. Now $M_{k} \backslash N^{\prime}=\Delta_{A}\left(N \backslash N^{\prime}\right)$ and $N \backslash N^{\prime}=M_{k-1}$, so $M_{k} \backslash N^{\prime}=$ $\Delta_{A}\left(M_{k-1}\right)$. But $\Delta_{A}\left(M_{k-1}\right)=\Delta_{A}\left(\nabla_{A}(Q)\right)=Q$ by Lemma 3.2.4. Now $M \backslash N^{\prime}$ has a minor with a path sequence that has fewer $\Delta$ - $\nabla$-steps than $M$, so by induction $M \backslash N^{\prime}$ has an $\mathscr{S}$-minor. Hence $M$ has an $\mathscr{S}$-minor.

We will prove that the class of $\mathbb{U}_{2^{-}}$or $\mathbb{H}_{5}$-representable fragile matroids with an $\left\{X_{8}, Y_{8}, Y_{8}^{*}, M_{8,6}\right\}$-minor is contained in $\mathscr{P}$. Note that it follows from Theorem 3.1 .1 that $\mathbb{H}_{5}$-representable fragile matroids with an $M_{8,6}$-minor but no $\left\{X_{8}, Y_{8}, Y_{8}^{*}\right\}$-minor are all in $\mathscr{P}$.

Let $M$ be a $\mathbb{P}$-representable fragile matroid $M$ with an $\left\{X_{8}, Y_{8}, Y_{8}^{*}\right\}$-minor. Suppose that $M$ is not in the class $\mathscr{P}$, and that $M$ is minimum-sized with respect to this property. Then $M$ is 3 -connected because $\mathscr{P}$ is closed under series-parallel extensions. Moreover, the dual $M^{*}$ is also not in $\mathscr{P}$ because $\mathscr{P}$ is closed under duality. It is easy to see that $M$ must have at least 10 elements. Thus, by the Splitter Theorem and duality, we can assume there is some element $x$ of $M$ such that $M \backslash x$ is also a 3 -connected $\mathbb{P}$-representable fragile matroid with an $\left\{X_{8}, Y_{8}, Y_{8}^{*}\right\}$ minor. By the assumption that $M$ is minimum-sized with respect to being outside the class $\mathscr{P}$, it follows that $M \backslash x \in \mathscr{P}$. Thus $M \backslash x$ has a path sequence $M_{1}, \ldots, M_{n}$. We show that there are three possibilities for the structure of $x$ in $M$ relative to the path of 3-separations associated with $M_{1}, \ldots, M_{n}$.

Let $(A, B)$ be a $k$-separation of $M \backslash x$. Recall that $x$ blocks $(A, B)$ if neither $(A \cup x, B)$ nor $(A, B \cup x)$ is a $k$-separation of $M$. We use the following characterisation of blocking.

Proposition 3.5.2. [9] Proposition 3.5] Let $(A, x, B)$ be a partition of $M$. If $(A, B)$ 
is an exact $k$-separation of $M \backslash x$, then $x$ blocks $(A, B)$ if and only if $x$ is not a coloop of $M, x \notin \mathrm{cl}_{M}(A)$ and $x \notin \mathrm{cl}_{M}(B)$.

We also use the following consequence of orthogonality.

Lemma 3.5.3. Let $(X,\{e\}, Y)$ be a partition of $E(M)$. Then $e \in \operatorname{cl}(X)$ if and only if $e \notin \mathrm{cl}^{*}(Y)$.

We can now locate $x$.

Lemma 3.5.4. Let $M$ and $M \backslash x$ be 3-connected $\left\{U_{2,5}, U_{3,5}\right\}$-fragile matroids. If $M \backslash x$ has a path sequence with associated path of 3-separations $\boldsymbol{P}$, then either:

(i) $x$ is in the guts of some 3-separation displayed by $\boldsymbol{P}$; or

(ii) x blocks some 3-separation displayed by $\boldsymbol{P}$; or

(iii) for each 3-separation $(R, G)$ of $M$ displayed by $\boldsymbol{P}$, there is some $X \in\{R, G\}$ such that $x \in \mathrm{cl}_{M}(X)$ and $x \in \mathrm{cl}_{M}^{*}(X)$.

Proof. Assume that neither (i) nor (ii) holds, and let $(R, G)$ be a 3-separation of $M \backslash x$ displayed by a maximal path-generating set. Then, by Lemma 3.5.2, we may assume that $x \in \mathrm{cl}_{M}(R)$. Now $x \notin \mathrm{cl}_{M}(G)$ because (ii) does not hold, so $x \in \mathrm{cl}_{M}^{*}(R)$ by Lemma 3.5.3. Thus (iii) holds.

We consider the three possible cases of Lemma 3.5.4 in the next three sections respectively. In case (i) we will obtain a direct contradiction to the assumption that $M \notin \mathscr{P}$. In cases (ii) and (iii) we bound the size of $M$ so that $M$ is at most a 10 -element matroid. This reduces the proof to a finite case-analysis, from which the contradiction to the assumption that $M \notin \mathscr{P}$ is then obtained by the following lemma.

Lemma 3.5.5. Let $M$ be a 3-connected $\mathbb{H}_{5}$-representable fragile matroid with an $\left\{X_{8}, Y_{8}, Y_{8}^{*}\right\}$-minor. If $|E(M)| \leq 10$, then $M \in \mathscr{P}$.

We defer the proof of Lemma 3.5.5 to Section 3.9. 


\subsection{Guts case}

First show that $x$ is not in the guts of an end 4-segment.

Lemma 3.6.1. If $M \backslash x$ has a 4-segment end $S$, then $x \notin \operatorname{cl}_{M}(S)$.

Proof. Suppose that $M \backslash x$ has a 4-segment end $S$, and that $x \in \operatorname{cl}_{M}(S)$. Then $M$ has a $U_{2,5}$-restriction $S \cup\{x\}$, so $y \in E(M)-\operatorname{cl}_{M}(S \cup x)$ is flexible; a contradiction because $M$ is fragile.

Next we show that $M \backslash x$ cannot be obtained from a path sequence ending in a 4-element segment or cosegment.

Lemma 3.6.2. There is no path sequence for $M \backslash x$ ending in a 4-element segment or cosegment.

Proof. Suppose there is some path sequence $M_{1}, \ldots, M_{n}$ for $M \backslash x$ such that the end step uses a 4-element segment or cosegment $A$. Then $A$ is an allowable 4element segment or cosegment of $M$ by Lemma 3.2.12 and Lemma 3.2.13, so $\Delta_{A}(M)$ or $\nabla_{A}(M)$ is well-defined. Moreover, it follows from Lemma 3.2 .9 that $\Delta_{A}(M) \backslash x=\Delta_{A}(M \backslash x)$ or $\nabla_{A}(M) \backslash x=\nabla_{A}(M \backslash x)$, which is an allowable series or parallel extension of $M_{n-1}$. Now, there is some element $a^{\prime} \notin A \cup x$ such that $a^{\prime}$ is in series or parallel with an element of $A$. Let $M^{\prime}=\Delta_{A}(M) / a^{\prime}$ if $a^{\prime}$ is in series with an element of $A$ and $M^{\prime}=\nabla_{A}(M) \backslash a^{\prime}$ if $a^{\prime}$ is in parallel with an element of $A$. Then $\left|E\left(M^{\prime}\right)\right|<|E(M)|$, so $M^{\prime} \in \mathscr{P}$ by the minimality of $M$. But $A$ is an allowable 4-element segment or 4-cosegment of $M^{\prime}$, so there is a path sequence for $M^{\prime}$ and we can assume that the path sequence ends in $A$. But then an allowable series-parallel extension along $A$ by $a^{\prime}$ followed by a 4 -element $\Delta$ - $\nabla$-move gives a matroid isomorphic to $M$ that is in $\mathscr{P}$; a contradiction because $M \notin \mathscr{P}$.

Next we deal with fans.

Lemma 3.6.3. There is no path sequence for $M \backslash x$ ending in a fan with at least 4-elements.

Proof. Suppose that there is some sequence $M_{1}, \ldots, M_{n}$ for $M \backslash x$ such that $n \geq 2$, and $M_{n}$ is obtained from $M_{n-1}$ by gluing a non-trivial fan $F$ onto an allowable triangle or triad of $M_{n-1}$. 
Then $F$ contains an allowable triad $T$ of $M \backslash x$, and $T$ is also an allowable triad of $M$ by Lemma 3.2.13. Then $\nabla_{T}(M)$ is an allowable parallel-extension of a matroid $M^{\prime}$, and $M^{\prime} \in \mathscr{P}$ by the minimality of $M$. Thus there is a path sequence for $M^{\prime}$, and that ends in a fan containing $T$ or a 4-segment containing $T$. But then this path sequence can extended to a path sequence for $M$, so $M \in \mathscr{P}$; a contradiction.

We can now finish off this case.

Lemma 3.6.4. $x$ is not in the guts of a 3-separation displayed by the path of 3separations associated with a path sequence for $M \backslash x$.

Proof. Seeking a contradiction, suppose that $x$ is in the guts of a 3-separation of $M \backslash x$. Then it follows from Lemma 3.6.3 and Lemma 3.6.2 that any path sequence for $M \backslash x$ ends in a triad or triangle. Moreover, since $|E(M \backslash x)| \neq 8$, the path sequence for $M \backslash x$ must have at least one $\Delta$ - $\nabla$-step.

Suppose that $M_{1}, \ldots, M_{n}$ is a path sequence for $M \backslash x$ that ends in a triad. By rearrangement, we can assume that the $(n-1)$-th step uses $A^{\prime}$, that $M_{n-1}$ has a 4-segment $A^{\prime}$, and $M_{n}=\Delta_{A}\left(M_{n-1}\right)$ for some allowable triangle $A \subseteq A^{\prime}$ of $M_{n-1}$. Now since $x$ is a guts element, it follows from Lemma 3.2.13 that $A$ is an allowable triad of $M$, so the matroid $\nabla_{A}(M)$ is well-defined. Moreover, by Lemma 3.2.9 $\nabla_{A}(M) \backslash x=\nabla_{A}(M \backslash x)$, so $A$ is contained in an allowable 4-segment $A^{\prime}$ of $\nabla_{A}(M)$ by Lemma 3.2.12. Now if $\nabla_{A}(M)$ is in $\mathscr{P}$, then it has a path sequence ending in $A^{\prime}$. But then this path sequence can be extended to a path sequence for $M$; a contradiction. Therefore $\nabla_{A}(M)$ is also a minimum-sized counterexample with $x$ in the guts. But $\nabla_{A}(M) \backslash x=M_{n-1}$ has a non-trivial path sequence $M_{1}, \ldots, M_{n-1}$ ending in a 4-segment; a contradiction of Lemma 3.6.2.

Now suppose that both ends of $M \backslash x$ are triangles. We may assume that $M_{1}, \ldots, M_{n}$ is a path sequence for $M \backslash x$ such that $n \geq 3, M_{n-1}$ has a path sequence ending in a 4-cosegment $A^{\prime}$, and $M_{n}=\nabla_{A}\left(M_{n-1}\right)$ for some allowable triad $A \subseteq A^{\prime}$ of $M_{n-1}$. We can assume, up to rearrangement, that the path sequence has been chosen such that $x \notin \mathrm{cl}_{M}(A)$. Now $A$ is an allowable triangle of $M$ by Lemma 3.2.12, so $\Delta_{A}(M)$ is well-defined and $A$ is contained in a 4-cosegment $A^{\prime}$ of $\Delta_{A}(M)$. If $\Delta_{A}(M) \in \mathscr{P}$, then it has a path sequence ending in $A^{\prime}$, and the path sequence can be extended to obtain $M$; a contradiction. Thus $\Delta_{A}(M)$ is a minimum-sized counterexample and $x$ is a guts element in $\Delta_{A}(M) \backslash x=\Delta_{A}(M \backslash x)=M_{n-1}$. But $M_{n-1}$ has a non-trivial 
path sequence $M_{1}, \ldots, M_{n-1}$ ending in a 4-cosegment; a contradiction of Lemma 3.6 .2

\subsection{Blocking case}

In this section, we assume that there is some $\mathbb{P}$-representable fragile matroid $M$ with an $\left\{X_{8}, Y_{8}, Y_{8}^{*}, M_{8,6}\right\}$-minor, and an element $x$ of $M$ such that:

(i) $M \backslash x$ is 3-connected and $M \backslash x \in \mathscr{P}$; and

(ii) $x$ blocks some 3-separation displayed by the path of 3-separations $\mathbf{P}$ associated with a path sequence for $M \backslash x$.

We may assume that $M$ is minimum-sized with respect to these properties. The fact that $M$ is minimum-sized gives the following straightforward restriction on the elements of $E(M)-\{x\}$.

Lemma 3.7.1. There is no element $y \in E(M)-\{x\}$ such that:

(i) $y$ is $N$-deletable in $M \backslash x, M \backslash x, y$ is 3-connected, $M \backslash x, y \in \mathscr{P}$, and $x$ blocks some 3-separation displayed by the path of 3-separations $\boldsymbol{P}^{\prime}$ associated with a path sequence for $M \backslash x, y$; or

(ii) $y$ is $N$-contractible in $M \backslash x, M \backslash x / y \in \mathscr{P}$, and $x$ blocks some 3-separation displayed by the path of 3-separations $\boldsymbol{P}^{\prime}$ associated with a path sequence for $\operatorname{si}(M \backslash x / y)$.

We make the following observations about rank that we will use for finding elements that can be contracted while preserving the property that $x$ blocks a 3separation.

Lemma 3.7.2. Let $M$ be a matroid in $\mathscr{P}$, and $A \subseteq E(M)$.

(i) If $A$ is an allowable 4-cosegment of $M$, and $R \subseteq E(M)-A$ is a 3-separating set in $M$, then $r\left(R \cup\left\{a, a^{\prime}\right\}\right)=r(R)+2$ for all distinct pairs $a, a^{\prime} \in A$.

(ii) If $A=\left(a_{1}, \ldots, a_{n}\right)$ is a fan with $n \geq 5$ such that the ends of $A$ are rim elements, and $R \subseteq E(M)-A$ is a 3-separating set in $M$, then $r\left(R \cup\left\{a_{1}, a_{n}\right\}\right)=$ $r(R)+2$. 
We first show that the path of 3-separations of $M \backslash x$ does not have large fan ends.

Lemma 3.7.3. $M \backslash x$ has no path sequence ending in a fan with at least 4 elements.

Proof. Suppose that $M \backslash x$ has a path sequence ending with a maximal fan $F$ of $M \backslash x$ such that $|F| \geq 4$. Suppose that $s \in F$ is a spoke end of $F$. Then $s$ is deletable in $M \backslash x, M \backslash x, s$ is 3-connected, $M \backslash x, s \in \mathscr{P}$, and $x$ blocks some 3-separation of $M \backslash s$, so $M$ has an element forbidden by Lemma 3.7.1 (i); a contradiction. Thus we may assume that the ends of $F$ are rim elements. Then by Lemma 3.7.2(ii) at least one of these ends $r$ satisfies $r \notin \mathrm{cl}_{M}((E(M)-F) \cup x)$, so $r$ is an element that is forbidden by Lemma 3.7.1 (ii); a contradiction.

Removing elements such that membership in $\mathscr{P}$ is preserved is important here. We now prove some technical results that enable us to find elements with the desired property. The first observation is on 4-segment and, by duality, for 4cosegment ends.

Lemma 3.7.4. Let $M \in \mathscr{P}$ be a 3 -connected matroid. If $M$ has a path sequence $M_{1}, \ldots, M_{n}$ that ends in a 4-segment $A$ of $M$, then there is some a $\in A$ such that $M \backslash a$ is 3-connected and $M \backslash a \in \mathscr{P}$.

Proof. Suppose that $M$ has a path sequence $M_{1}, \ldots, M_{n}$, where $A$ a 4-cosegment of $M_{n-1}$ and $M_{n}=\nabla_{A}^{Q}\left(M_{n-1}\right)$. Let $a \in A$ be an element that is in series with an element $q \in E(Q)-E\left(M_{n-1}\right)$ in $Q$. Then $M \backslash a$ is 3 -connected since $A$ is a 4 segment of $M$, and $M \backslash a \cong \nabla_{A-a}^{Q / q}\left(M_{n-1}\right)$. But then $M \backslash a$ is obtained from $M_{n-1}$ by a fan move on the allowable $\operatorname{triad} A-a$ of $M_{n-1}$, so $M \backslash a \in \mathscr{P}$.

Next we consider triad and triangle ends.

Lemma 3.7.5. Let $M \in \mathscr{P}$ be a 3-connected matroid with a path sequence $M_{1}, \ldots, M_{n}$ with a $\Delta$ - $\nabla$-step on $A$ and a triangle end $A^{\prime} \subseteq A$. If $M$ has no path sequence where $A^{\prime}$ is contained in a larger fan or 4-segment, then

(i) $M \backslash a^{\prime}$ is 3-connected and $M \backslash a^{\prime} \in \mathscr{P}$ for some $a^{\prime} \in A^{\prime}$; or

(ii) $M / a$ is 3-connected and $M / a \in \mathscr{P}$ for $a \in A-A^{\prime}$. 
Proof. Since $A^{\prime}$ is not contained in a larger fan, it follows that $M \backslash a^{\prime}$ is 3-connected for each deletable element $a^{\prime} \in A$. Suppose that $M \backslash a^{\prime} \notin \mathscr{P}$ for all deletable elements $a^{\prime} \in A^{\prime}$. Now since there is a $\Delta-\nabla$-step on $A$ we can assume by rearrangement that the path sequence has the form $M_{n-1}=\Delta_{A}^{N}\left(M_{n-2}\right)$ and $M_{n}=\nabla_{A^{\prime}}\left(M_{n-1}\right)$. So $M \backslash a^{\prime}$ is $M_{n-1} / a^{\prime}=\Delta_{A-a^{\prime}}\left(N \backslash a^{\prime}\right)$. Since $M \backslash a^{\prime}$ is not in $\mathscr{P}$, it follows that $N \backslash a^{\prime}$ must not be an allowable extension of $M_{n-2}$ for each deletable $a^{\prime} \in A^{\prime}$. But $N$ must add some element in parallel with a deletable element of $A$, so it must be the element $a \in A-A^{\prime}$. It follows from Lemma 3.2.4 and Lemma 3.2.8 that $M_{n} / a=N \backslash a$. Now $N \backslash a$ is $M_{n-2}$ because $a$ was the only element in a parallel class of $N$, so $M_{n} / a$ is 3-connected and $M_{n} / a \in \mathscr{P}$.

If a path sequence has a subsequence of the form $M_{i}=\Delta_{A}^{Q}\left(M_{i-1}\right)$ and $M_{i+1}=$ $\nabla_{A}^{R}\left(M_{i}\right)$, then $r \in E(R)-E\left(M_{i}\right)$ is called an internal coguts element and $q \in$ $E(Q)-E\left(M_{i-1}\right)$ is called an internal guts element. We prove a lemma about removing internal guts and coguts elements.

Lemma 3.7.6. Let $M \in \mathscr{P}$ be a 3-connected matroid with a path sequence $M_{1}, \ldots, M_{n}$ with $\Delta$ - $\nabla$-steps on $A$. If $q \in E(M)$ is an internal guts element and $r \in E(M)$ is an internal coguts element, then $M \backslash q, M / r \in \mathscr{P}$.

Proof. Suppose some subsequence of steps of the path sequence has the form $M_{i}=\Delta_{A}^{Q}\left(M_{i-1}\right)$ and $M_{i+1}=\nabla_{A}^{R}\left(M_{i}\right)$. Let $r \in E(R)-E\left(M_{i}\right)$. Then either $R / r=M_{i}$ or $R / r$ is an allowable series extension of $M_{i}$ along $A$. Suppose first that $R / r=M_{i}$. Then $M_{i+1} / r=Q$ by Lemma 3.2.4, so $M_{i+1} / r \in \mathscr{P}$. Now consider contracting the element of $M$ corresponding to $r$. The contraction operation commutes with subsequent steps of the path sequence for $M$ by Lemma 3.2.9, so $M / r \in \mathscr{P}$ too. For the second case, suppose that $R / r$ is an allowable series extension of $M_{i}$. Consider contracting $r$ from $M$. Again the contraction of $r$ commutes with any subsequent steps of the path sequence for $M$ by Lemma 3.2.9, and the $(i+1)$-th step becomes $\nabla_{A}^{R / r}\left(M_{i}\right)$. It follows that $M / r \in \mathscr{P}$.

For $q \in Q-M_{i-1}$, either $Q \backslash q=M_{i-1}$ or $Q \backslash q$ is an allowable parallel extension of $M_{i-1}$. Suppose that $Q \backslash q=M_{i-1}$. Then $M_{i} \backslash q=\Delta_{A}\left(M_{i-1}\right)$ by Lemma 3.2.4. Now $M_{i+1} \backslash q$ is equal to $M_{i-1}$ up to series pairs by Lemma 3.2.4, and the deletion of $q$ commutes with subsequent steps of the path sequence for $M$ by Lemma 3.2.9. so $M \backslash q \in \mathscr{P}$. Suppose that $Q \backslash q$ is an allowable parallel extension of $M_{i-1}$. Then the deletion of $q$ commutes with any subsequent steps of the path sequence for 
$M$ by Lemma 3.2.9, and the $(i+1)$-th step becomes $\Delta_{A}^{Q \backslash q}\left(M_{i-1}\right)$. It follows that $M \backslash q \in \mathscr{P}$.

Next we eliminate 4-segments.

Lemma 3.7.7. $M \backslash x$ has no path sequence such that the end step is a 4-segment $A$ of $M \backslash x$.

Proof. Suppose that $M \backslash x$ has a path sequence $M_{1}, \ldots, M_{n}$ such that $A$ a 4 cosegment of $M_{n-1}$ and $M_{n}=\nabla_{A}^{Q}\left(M_{n-1}\right)$. Let $a \in A$ be an element that is in series with an element $q \in E(Q)-E\left(M_{n-1}\right)$ in $Q$. Then $M \backslash x, a$ is 3-connected since $A$ is a 4-segment of $M \backslash x$, and $M \backslash x, a \cong \nabla_{A-a}^{Q / q}\left(M_{n-1}\right)$. So $M \backslash x, a$ is in $\mathscr{P}$. But $x$ still blocks a 3 -separation displayed by the path of 3-separations generated by $A-a$. Thus $a$ is an element that is forbidden by Lemma 3.7.1(i); a contradiction.

Lemma 3.7.8. $x$ does not block a 3-separation displayed by the path of 3separations associated with a path sequence for $M \backslash x$.

Proof. Suppose that $M \backslash x$ has a path sequence $M_{1}, \ldots, M_{n}$. We may assume by Lemma 3.7 .3 and Lemma 3.7 .7 that $M \backslash x$ has no path sequence that ends with a non-trivial fan or 4-segment.

3.7.8.1. $M_{1}, \ldots, M_{n}$ has at least two $\Delta$ - $\nabla$-steps.

Subproof. We consider the cases for the end step. Suppose that $M \backslash x$ has a path sequence $M_{1}, \ldots, M_{n}$ that ends in a triangle. Suppose the path sequence for $M \backslash x$ has no $\Delta$ - $\nabla$-steps. Then, since $M \backslash x$ has no path sequence ending in a fan with at least 4 elements, it follows that $|M \backslash x|=8$, and so $|M|=9$; a contradiction by inspection of the 9-element catalogue. Thus, by rearrangement, we can assume that $M \backslash x$ has a path sequence $M_{1}, \ldots, M_{n}$ such that $A$ a 4-segment of $M_{n-2}, M_{n-1}=\Delta_{A}^{N}\left(M_{n-2}\right)$, and finally $M_{n}=\nabla_{A^{\prime}}\left(M_{n-1}\right)$ for some allowable triad $A^{\prime} \subseteq A$ of $M_{n-1}$. Now it follows from Lemma 3.7 .5 that either $M \backslash x, a^{\prime}$ is 3-connected and $M \backslash x, a^{\prime} \in \mathscr{P}$ for some $a^{\prime} \in A^{\prime}$, or else $M \backslash x / a$ is 3-connected and $M \backslash x / a \in \mathscr{P}$ for $a \in A-A^{\prime}$. Suppose that $M \backslash a^{\prime}$ is 3-connected and $M \backslash a^{\prime} \in \mathscr{P}$ for some $a^{\prime} \in A^{\prime}$. Then $a^{\prime}$ is an element forbidden by Lemma 3.7.1 (i); a contradiction. We may therefore assume that $M \backslash x / a$ is 3 -connected and $M \backslash x / a \in \mathscr{P}$ for $a \in A-A^{\prime}$. Since $M \backslash x / a$ is 3-connected it follows that $\left|E(N)-E\left(M_{n-2}\right)\right|=1$. Now if there is only one 
$\Delta$ - $\nabla$-step in the path sequence $M_{1}, \ldots, M_{n}$, then it follows that $|M \backslash x|=9$; a contradiction because there is no such matroid in the 9-element catalogue. Thus the path sequence $M_{1}, \ldots, M_{n}$ has at least two $\Delta$ - $\nabla$-steps.

Suppose that the path sequence $M_{1}, \ldots, M_{n}$ ends in a 4-element cosegment end $A$, so $M_{n}=\Delta_{A}^{N}\left(M_{n-1}\right)$. We may assume that $M \backslash x$ has no triangle end by the above argument. Now $\left|E(N)-E\left(M_{n-1}\right)\right|=1$ or there are at least two elements $a, a^{\prime} \in A$ such that $M \backslash x / a$ and $M \backslash x / a^{\prime}$ are 3-connected and $M \backslash x / a, M \backslash x / a^{\prime} \in \mathscr{P}$, and $x$ blocks a 3-separation of at least one of $M \backslash x / a$ and $M \backslash x / a^{\prime}$ by Lemma 3.7.2; a contradiction of Lemma 3.7.1 (ii). Thus there is exactly one $a \in A$ such that $M \backslash x / a$ is 3 -connected and $M \backslash x / a \in \mathscr{P}$. Now if the path sequence $M_{1}, \ldots, M_{n}$ has only one $\Delta-\nabla$-step, then $|M \backslash x|=9$; a contradiction because there is no such matroid in the 9-element catalogue. It follows that there are at least two $\Delta$ - $\nabla$-steps in the path sequence $M_{1}, \ldots, M_{n}$.

Suppose that the path sequence $M_{1} \ldots M_{n}$ with a $\Delta-\nabla$-step that ends in a triad. By rearrangement, we can assume that $A$ a 4-cosegment of $M_{n-2}, M_{n-1}=\nabla_{A}^{N}\left(M_{n-2}\right)$, and finally $M_{n}=\Delta_{A^{\prime}}\left(M_{n-1}\right)$ for some allowable triangle $A^{\prime} \subseteq A$. We can assume that $M \backslash x$ has no path sequence ending in a end fan, 4-segment or 4-cosegment, or triangle. Now $\left|E(N)-E\left(M_{n-2}\right)\right|=1$ or $M \backslash x$ contradicts Lemma 3.7.1 (ii), since at most one of $E(N)-E\left(M_{n-2}\right)$ can lose the blocking property by Lemma 3.7.2. Thus, if the path sequence $M_{1} \ldots M_{n}$ has one $\Delta$ - $\nabla$-step, then $|M \backslash x|=9$. It follows from the 9-element catalogue that $M \backslash x=M_{9,17}$. But then $M$ is not a fan extension of $M_{7,1}$, so by Theorem 3.1.1 $M$ has an $\left\{X_{8}, Y_{8}, Y_{8}^{*}\right\}$-minor. Then $M \in \mathscr{P}$ by Lemma 3.5.5. We may therefore assume that the path sequence $M_{1} \ldots M_{n}$ has at least two $\Delta-\nabla$-steps.

Now $M_{1}, \ldots, M_{n}$ has at least two $\Delta$ - $\nabla$-steps, and by rearrangement we can assume they use $A$, and that either $A$ is an end 4-cosegment of $M \backslash x$ or contains an end triangle or triad $A^{\prime} \subseteq A$. Let $B=E(M \backslash x)-A$.

3.7.8.2. $x$ blocks the 3-separation $(A, B)$.

Subproof. We show that $x \notin \mathrm{cl}_{M}(A)$ and $x \notin \mathrm{cl}_{M}(B)$. Since $x$ blocks some 3separation displayed by the path of 3-separations generated by the end step $A$ or $A^{\prime}$, it follows from Lemma 3.5.2 that $x \notin \mathrm{cl}_{M}(A)$. Now, by Lemma 3.7.4 and Lemma 3.7.5, there is some $a \in A$ such that $M \backslash x / a \in \mathscr{P}$. Then it follows from Lemma 3.7.1 (ii) that $x$ does not block a 3-separation of $M \backslash x / a \in \mathscr{P}$ generated 
by $A-a$. In particular, $x$ does not block $(A-a, B)$ in $M / a$, so it follows that $a \in \mathrm{cl}_{M}(B \cup x)$. But $a \notin \mathrm{cl}_{M \backslash x}(B)$, so $x \notin \mathrm{cl}_{M}(B)$ too. The claim now follows from Lemma 3.5.2.

Now, up to rearragement, we may assume that $M \backslash x$ has a path sequence $M_{1} \ldots M_{n}$ with at least two $\Delta-\nabla$-steps on $A$, and an internal guts element $q \in B$ and an internal coguts element $r \in B$. By Lemma 3.7.6 $M \backslash x, q \in \mathscr{P}$ and $M \backslash x / r \in \mathscr{P}$. Now by Lemma 3.7.1 (ii) $x$ does not block a 3-separation of $M \backslash x / r$, so in particular $x$ does not block $(A, B-r)$. Hence $r \in \operatorname{cl}_{M}(A \cup x)$ by Lemma 3.5.2. By Lemma 3.7.1 (i) $M \backslash x, q$ is not 3-connected, so $M \backslash x, q$ has a series pair $S$. Moreover, $S-(A \cup r) \neq \emptyset$ because $q$ is a guts element of the path generated by $A$. Let $s \in S-(A \cup r)$. Then $M \backslash x / s \in \mathscr{P}$, and so $x$ does not block the 3-separation $(A, B-s)$ of $M / s$. Then $s \in \mathrm{cl}_{M}(A \cup x)$ by Lemma 3.5.2, so $r, s \in \mathrm{cl}_{M}(A \cup x)$; a contradiction of Lemma 3.7 .2 .

\subsection{Spanning and cospanning case}

Assume that, for every 3-separation $(R, G)$ displayed by a path-generating set of $M \backslash x$, there is some $S \in\{R, G\}$ such that $x \in \operatorname{cl}_{M}(S)$ and $x \notin \mathrm{cl}_{M}^{*}(S)$.

Let $M$ be a minimum-sized counterexample. That is, let $M$ be a fragile matroid such that $M \notin \mathscr{P}$, and $x \in E(M)$ an element such that $M \backslash x \in \mathscr{P}$ is a 3-connected fragile matroid and outcome (iii) of Lemma 3.5 .4 holds.

Lemma 3.8.1. If $M \backslash x$ has a path sequence $M_{1}, \ldots, M_{n}$ with end step $A$, then we may assume that either:

(i) A is a 4-cosegment, triad, or non-trivial fan that spans and cospans $x$; or

(ii) A is a 4-segment or triangle such that $\mathrm{cl}^{*}(A)$ spans and cospans $x$.

Proof. Assume that $A$ is a 4-element cosegment of $M \backslash x$. Suppose $x$ is not spanned and cospanned by $A$. Then $A$ is an allowable 4-cosegement of $M$ by Lemma 3.2.13, and $Z=\operatorname{cl}_{M}(A)-A$ is non-empty. Now $\nabla_{A}(M) \backslash Z, x \in \mathscr{P}$ and $\nabla_{A}(M) \backslash Z \in \mathscr{P}$ since $M$ is minimum-sized, so then there is a path-sequence for $\nabla_{A}(M) \backslash Z$ ending in $A$. But then $M$ is in $\mathscr{P}$; a contradiction. Thus (i) holds when $A$ is a 4-cosegment. 
Assume that $A$ is a non-trivial fan of $M \backslash x$. Suppose $x$ is not spanned and cospanned $A$. Then there is an an allowable triad $T \subseteq A$ of $M$ by Lemma 3.2.13. Let $a \in$ $\operatorname{cl}_{M}(T)$. Then $a$ is in parallel with an element of $T$ in $\nabla_{T}(M)$, and $\nabla_{T}(M) \backslash a \in \mathscr{P}$ by the minimality of $M$. But then we can extend the path sequence for $\nabla_{T}(M) \backslash a$ to a path sequence for $M$, so $M \in \mathscr{P}$; a contradiction. Thus (i) holds when $A$ is a non-trivial fan.

Assume that $A$ is a triad of $M \backslash x$. Suppose that $x$ is not spanned and cospanned by $A$. Then $A$ is an allowable triad of $M$ by Lemma 3.2.13, and $\nabla_{A}(M)$ has a 4segment $A^{\prime}$ such that $A \subseteq A^{\prime}$. If $\nabla_{A}(M) \in \mathscr{P}$, then $\nabla_{A}(M)$ has a path sequence that ends in $A^{\prime}$, so $M \in \mathscr{P}$; a contradiction. Thus $\nabla_{A}(M) \notin \mathscr{P}$. By Lemma 3.2.9, we have $\nabla_{A}(M) \backslash x=\nabla_{A}(M \backslash x)$, so $\nabla_{A}(M)$ is a minimum-sized counterexample that ends in a 4-segment $A^{\prime}$.

Suppose that $A$ is a triangle, and let $B=E(M \backslash x)-\operatorname{cl}^{*}(A)$. Seeking a contradiction, suppose that $x \in \operatorname{cl}_{M}(B)$ and $x \in \mathrm{cl}_{M}^{*}(B)$. Then $N=\nabla_{A}(M)$ is also a minimumsized counterexample, and $\nabla_{A}(M) \backslash x=M_{n-1}$ has a 4-cosegment $A$; a contradiction of the above argument because $x \notin \operatorname{cl}_{M}(A)$ and $\operatorname{cl}_{M}(A)=\mathrm{cl}_{N}(A)$. Thus $\mathrm{cl}_{M}^{*}(A)$ spans and cospans $x$.

Suppose that $A$ is a 4-segment of $M \backslash x$, and let $B=E(M \backslash x)-\operatorname{cl}^{*}(A)$. Suppose that $x \in \operatorname{cl}_{M}(B)$ and $x \in \operatorname{cl}_{M}^{*}(B)$. Now $A$ is an allowable 4-segment of $M$, and $N=\Delta_{A}(M)$ is defined. Now $\Delta_{A}(M) \backslash x=\Delta_{A}(M \backslash x)$ by Lemma 3.2.9, and we still have $x \in \operatorname{cl}_{N}(B)$ and $x \in \mathrm{cl}_{N}^{*}(B)$. But $N \backslash x$ has at least one element $q \in Q$ in series with some element of $A$, and since $x \in \mathrm{cl}_{M}^{*}(B)$ it follows that $N$ has some element of $Q$ in series with some element of $A$. But then $N / q \in \mathscr{P}$ Hence it has a path sequence ending in $A$. But $N$ is an allowable extension of $A$, so $M=\nabla_{A}(N) \in \mathscr{P}$; a contradiction. Thus $\operatorname{cl}_{M}^{*}(A)$ spans and cospans $x$.

We now reduce to the spanning and cospanning fan end case.

Lemma 3.8.2. If $M \backslash x$ has no end fan, then $|M \backslash x|=8$.

Proof. Suppose that $M \backslash x$ has no fan ends. If the only moves are triangle or triad moves, then $|M \backslash x|=8$. We may assume that $M \backslash x$ has a path sequence with a 4-element $\Delta$ - $\nabla$-step. By Lemma 3.8.1 we can also assume that $M \backslash x$ has a pathsequence ending in a 4-segment, 4-cosegment, or a triangle. Now $M \backslash x$ cannot have a path sequence ending in a 4-cosegment $A$ or consecutive moves ending in a triangle $A^{\prime} \subseteq A$ or else $x$ is spanned and cospanned by both $A$ and the complement 
of $A$ by Lemma 3.8.1. By the same argument, no path sequence for $M \backslash x$ can have more than one step ending in a 4-segment. Thus the only remaining case to consider is when the path sequence has a single step ending in a 4-segment. Suppose that $M \backslash x=\nabla_{C}^{Q}\left(X_{8}\right)$, where $S$ and $C$ are the 4-element segment and cosegment of $X_{8}$ repsectively. Let $s \in S$. Then $M \backslash x, s$ is 3-connected and has a 4-segment $C$. By the minimality of $M$, it follows that $M \backslash s \in \mathscr{P}$, and since $C$ is a 4-segment of $M \backslash s$ there is a path sequence for $M \backslash s$ ending in $C$. But now $s$ is an element in the guts of a 3-separation displayed by the path of 3-separations for $M \backslash s$, so $M \in \mathscr{P}$ by Lemma 3.6.4; a contradiction. Therefore $M \backslash x$ has no path sequence with a 4-element $\Delta$ - $\nabla$-step, so $|M \backslash x|=8$.

Let $F$ be a fan of a matroid $M \backslash x$ with fan ordering $\left(f_{1}, \ldots, f_{n}\right)$. We say that $x$ is on a guts line of $F$ if there are fan elements $f_{i}$ and $f_{j}$ of $F$ such that $x$ is in the guts of the 3-separation $(R, G)$ of $M$, where $R=\left\{f_{i}, f_{i+1}, \ldots, f_{j-1}, f_{j}\right\}$.

We claim that if $x$ is on a guts line of a fan of $M \backslash x$, then $M$ is $\mathscr{P}$.

Lemma 3.8.3. Suppose that $M \backslash x \in \mathscr{P}$ has a path-generating fan $F$ with $|F| \geq 4$. If $A=\left\{x, f_{i}, f_{i+1}, f_{i+2}\right\}$ is a 4-segment of $M$ for some triangle $\left\{f_{i}, f_{i+1}, f_{i+2}\right\}$ of $M \backslash x$ contained in $F$, then $M \in \mathscr{P}$.

Proof. Since the rim element $f_{i+1}$ is contractible in $M \backslash x$, it follows that $f_{i+1}$ is contractible in $M$. Thus $f_{i+1}$ is non-deletable in $M$, so $A$ is a path-generating segment of $M$. Thus $\Delta_{A}(M)$ is fragile $\mathbb{P}$-representable matroid and $A$ is a pathgenerating cosegment of $\Delta_{A}(M)$ with non-contractible element $f_{i+1}$. Let $S$ be the set of elements of $M$ such that $S=\operatorname{cl}_{M}^{*}(A)-A$. Since $F$ is a fan of $M \backslash x$ with $|F| \geq 4$, it follows that $S$ is non-empty. Moreover, the members of $S$ are in series with contractible elements of $A$ because $A \cup S$ cannot contain a 5-point coline. Let $M_{R}=\Delta_{A}(M) / S$. Now $A$ is a path-generating cosegment of $M_{R}$ with noncontractible element $f_{i+1} \in A$, and $\operatorname{cl}_{M_{R}}(A)-A=\operatorname{cl}_{M}(A \cup S)-(A \cup S)$. Now $M_{R}^{*}$ has fan $F-S$ with $A$ a 4-segment of $M_{R}^{*}$, and $|F-S|<|F|$. If $F-S$ is a triangle, then there is a path sequence for $M_{R}^{*}$ ending in $A$ by the guts case. Assume that $|F-S| \geq 4$. By induction we may assume that there is some path sequence for $M_{R}^{*}$, and hence for $M_{R}$ ending in $A$. Then $\Delta_{A}(M)$ is an allowable series extension of $M_{R}$, so $M=\nabla_{A}\left(\Delta_{A}(M)\right)$ has a path sequence ending in $A$.

Lemma 3.8.4. If $x$ is on a guts line of an end fan of $M \backslash x$, then $M \in \mathscr{P}$. 
Proof. Suppose that $x$ belongs to a guts line of $F$. If the guts line is a triangle of $F$, then $M \in \mathscr{P}$ by Lemma 3.8.3. Suppose that $x$ is on the $\left\{f_{1}, f_{2}\right\}$ guts line of $F$, where $f_{1}$ a rim element, and $T=\left\{x, f_{1}, f_{2}\right\}$ is a triangle of $M$. Then $T$ is an allowable triangle of $M$, and $\Delta_{T}(M)$ has an allowable 4-point cosegment $A=T \cup\left\{f_{3}\right\}$. But then it follows from Lemma 3.8.3 that $\left(\Delta_{T}(M)\right)^{*} \in \mathscr{P}$, hence $M \in \mathscr{P}$.

We may therefore assume that $x$ is on an $f_{i}-f_{j}$ guts line for non-consecutive $i, j \in\{1, \ldots, n\}$. Since $x \in \operatorname{cl}_{M}\left(E(M \backslash x)-\left\{f_{i}, \ldots, f_{j}\right\}\right)$ it follows that $x \notin$ $\operatorname{cl}_{M}^{*}\left(\left\{f_{i}, \ldots, f_{j}\right\}\right)$, so an allowable triad $T=\left\{f_{k}, f_{f+1}, f_{k+2}\right\}$ of $M \backslash x$ that is contained in $\left\{f_{i}, \ldots, f_{j}\right\}$ is an allowable triad of $M$. Then $\nabla_{T}(M) \backslash\left\{f_{k-1}, f_{k+3}\right\}$ has $x$ on a guts line of the fan $F^{\prime}=F-\left\{f_{k-1}, f_{k+3}\right\}$. Now if $F^{\prime}=T$, then $F^{\prime} \cup x$ is a 4-segment and it follows from Lemma 3.8 .3 that there is a path sequence for $\nabla_{T}(M) \backslash\left\{f_{k-1}, f_{k+3}\right\}$ ending in $F^{\prime} \cup x$, and $M$ is obtained by gluing a fan onto $F^{\prime}$. Hence $M \in \mathscr{P}$. Assume $\left|F^{\prime}\right| \geq 4$. Then by induction $\nabla_{T}(M) \backslash\left\{f_{k-1}, f_{k+3}\right\}$ has a path sequence ending with the fan $F^{\prime}$. But $M$ is obtained by extending $F^{\prime}$ to $F$, so then $M \in \mathscr{P}$.

Thus if $M \notin \mathscr{P}$, then $x$ is not on a guts line. We now show that in this case $|F|$ must be small.

Lemma 3.8.5. If $M \backslash x$ has a maximal fan end $F$ such that $x \in \mathrm{cl}_{M}(F)$ and $x \in$ $\mathrm{cl}_{M}^{*}(F)$, then $|F|=4$ and $x$ is not on a guts line of $F$.

Proof. Suppose that $M \backslash x$ has a maximal fan end $F$ such that $x \in \mathrm{cl}_{M}(F)$ and $x \in \mathrm{cl}_{M}^{*}(F)$. Since $M \notin \mathscr{P}$, it follows from Lemma 3.8.4 that $x$ does not belong to a guts line of $F$. Suppose that $|F| \geq 5$. If $F$ has an end spoke element $s$, then it's easy to see that $M \backslash s$ is a fragile matroid with a fan $F-s$ such that $x \in \operatorname{cl}_{M \backslash s}(F-s)$ and $x \in \mathrm{cl}_{M \backslash s}^{*}(F-s) x$ is not on a guts line of $F-s$, so $M$ is not minimal with this property. We may therefore assume that $F$ has rim ends $r$ and $r^{\prime}$.

Now consider the 3-separations $F-r$ and $F-r^{\prime}$ of $M \backslash x$.

3.8.5.1. If $x \notin \mathrm{cl}_{M}(F-r)$ and $x \notin \mathrm{cl}_{M}\left(F-r^{\prime}\right)$, then $x$ blocks at least one of the 3-separations $(F-r, G \cup r)$ and $\left(F-r^{\prime}, G \cup r^{\prime}\right)$ of $M \backslash x$.

Subproof. Suppose that $x \notin \mathrm{cl}_{M}(F-r)$ and $x \notin \mathrm{cl}_{M}\left(F-r^{\prime}\right)$. Seeking a contradiction, suppose that $x \in \mathrm{cl}_{M}(G \cup r)$ and $x \in \operatorname{cl}_{M}\left(G \cup r^{\prime}\right)$. Now $x \notin \mathrm{cl}_{M}(G)$ because $x$ is not a guts element, so by closure exchange $r, r^{\prime} \in \mathrm{cl}_{M}(G \cup x)$; a contradiction 
because $r\left(G \cup\left\{r, r^{\prime}\right\}\right)=r(G)+2>r(G)+1=r(G \cup x)$. Therefore $x \notin \operatorname{cl}_{M}(G \cup r)$ or $x \notin \mathrm{cl}_{M}\left(G \cup r^{\prime}\right)$.

Suppose that $x \notin \mathrm{cl}_{M}(F-r)$ and $x \notin \mathrm{cl}_{M}\left(F-r^{\prime}\right)$. Then by 3.8.5.1 we may assume that $x$ blocks $(F-r, G \cup r)$. Since $|F| \geq 5$, there are at least two rim elements of $F-r$. Then by Lemma 3.7.2 there is some rim element $r^{\prime \prime}$ of $F-r$ such that $\operatorname{si}\left(M \backslash x / r^{\prime \prime}\right) \in \mathscr{P}$ and $x$ blocks a 3-separation of an end fan of $\operatorname{si}\left(M \backslash x / r^{\prime \prime}\right)$; a contradiction of the property that $M$ is minimum-sized with an element blocking some 3-separation of an end fan.

We may therefore assume that $x \in \operatorname{cl}_{M}(F-r)$. Now $x \notin \mathrm{cl}_{M}(G \cup r)$ since $x$ is not on a guts line of $F$. Now $M \backslash x / r \in \mathscr{P}$ and has an end fan $F-r$ such that $x$ is not contained in a guts line of $F-r$ in $M / r$; a contradiction because $M$ is minimum-sized with this property.

Thus a largest minimum-sized counterexample $M$ has 10 elements.

Lemma 3.8.6. $|M \backslash x| \leq 9$.

Proof. We may assume that $M \backslash x$ has an end fan $F$, and that $F$ spans and cospans $x$. By Lemma 3.8.5 $|F|=4$. A path sequence for $M \backslash x$ has no other steps. Thus $|M \backslash x|=9$.

\subsection{Case analysis}

We now prove that there is no 3-connected $\mathbb{H}_{5}$-representable fragile matroid $M$ with an $\left\{X_{8}, Y_{8}, Y_{8}^{*}\right\}$-minor with $|M| \leq 10$ that is not in $\mathscr{P}$.

Proof of Lemma 3.5.5. Suppose that $|E(M)|=8$. Then, up to duality, $M=X_{8}$ or $M=Y_{8}$. Now $X_{8} \in \mathscr{P}$ and $Y_{8}=\nabla_{T}\left(X_{8}\right)$ for any allowable triad $T \subseteq C$ of $X_{8}$, so $Y_{8} \in \mathscr{P}$. Suppose that $|E(M)|=9$. Then, up to duality, we may assume that $M \in\left\{M_{9,3}, M_{9,4}, M_{9,6}\right\}$. Then $M$ is obtained from $Y_{8}$ by gluing a wheel to some allowable triangle, so $M \in \mathscr{P}$. We may therefore assume that $|E(M)|=10$. Then, up to duality, $M$ is one of 21 matroids, and each of these matroids has a path sequence (see 3.9.1 and 3.9.2). Thus $M \in \mathscr{P}$. 


\subsubsection{Input}

The computation was carried out using Sage Mathematics Software [33]. In particular, making use of the Sage Matroid Package [29]. The files for accessing the catalog and the $\mathbb{H}_{5}$ product ring can be found on the arXiv in [2].

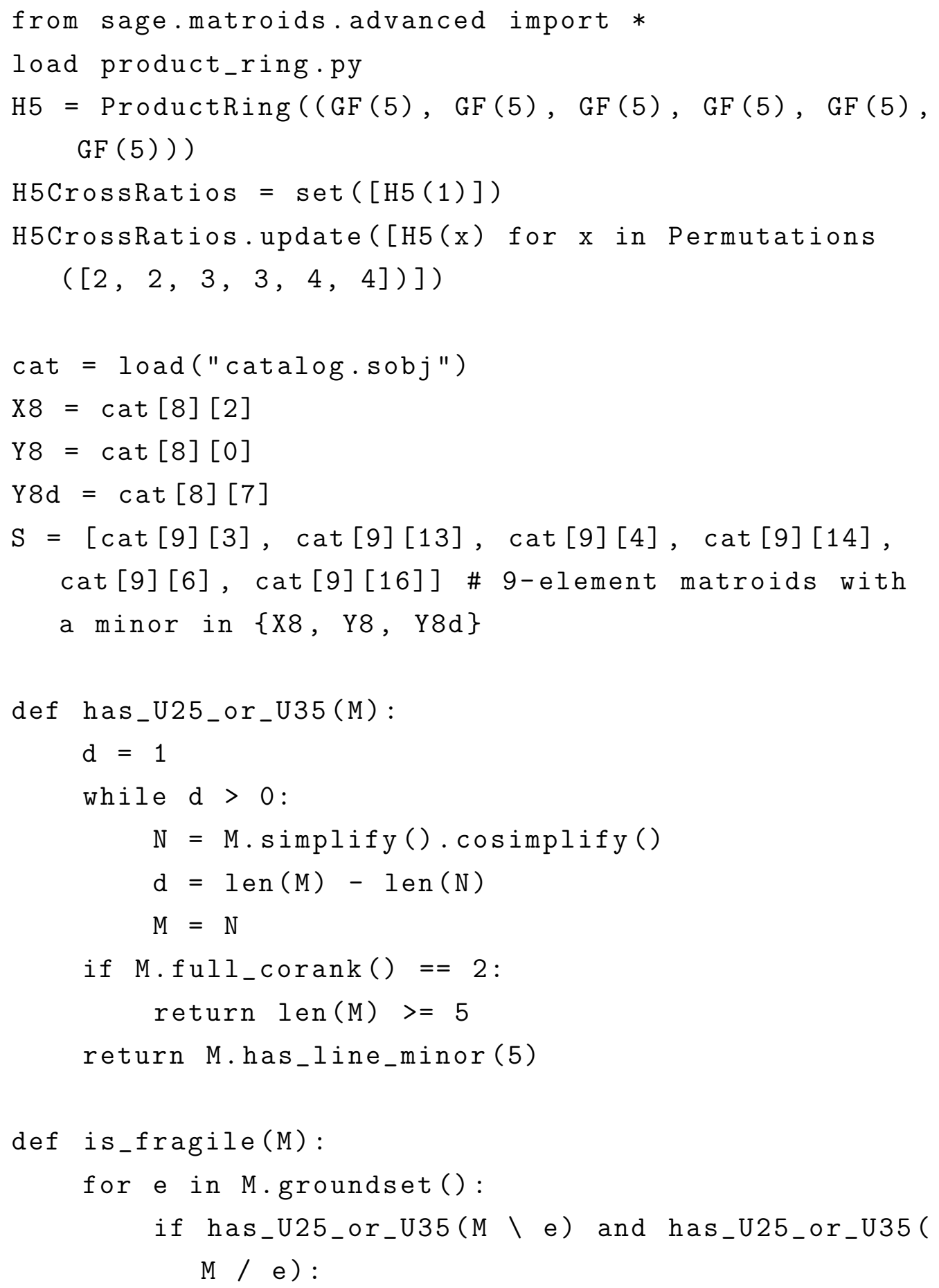




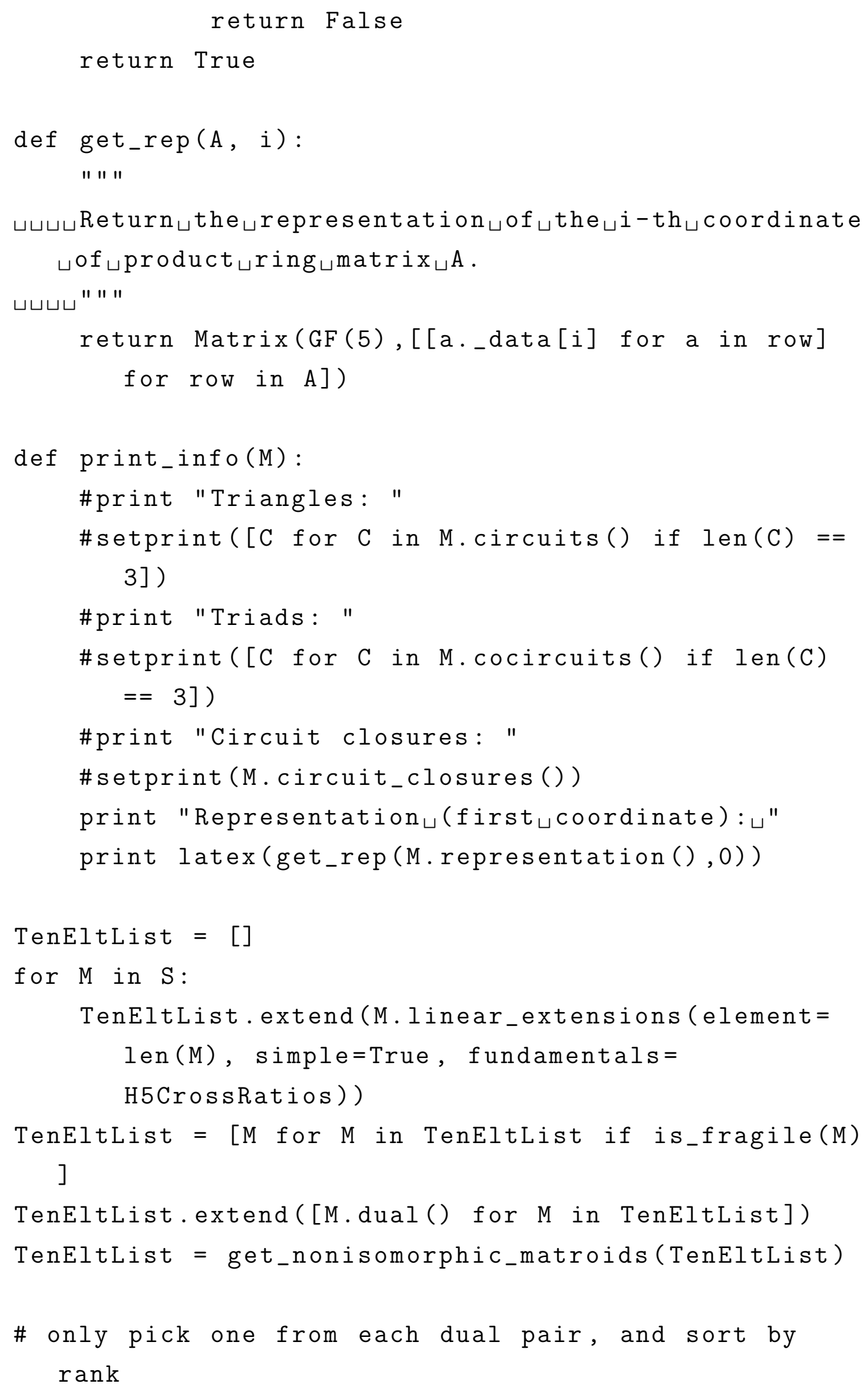




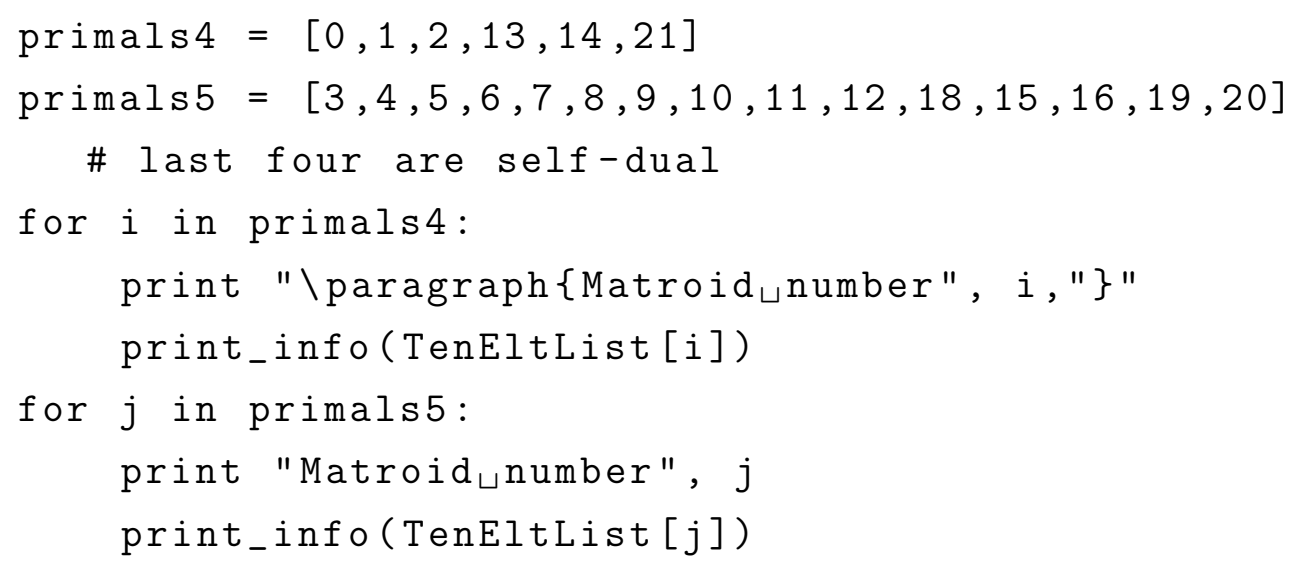

\subsubsection{Output}

The following matroids were generated by the computation 3.9.1. For each matroid, we give the $\mathrm{GF}(5)$-representation obtained from the $\mathbb{H}_{5}$-representation by projection onto the first coordinate. We then verify by hand that there is some path sequence for each of these matroids. We give the set used by the end step of a path sequence, where the matroid has the ground set $\{0,1,2, \ldots, 9\}$ and the element $i \in\{0,1,2, \ldots, 9\}$ labels the $i$ th column of the GF(5)-representation.

Matroid number 0. $M_{10,0}$ has path sequence ending with the fan $(1,0,6,8,9)$. $\mathrm{GF}(5)$-representation (first coordinate):

$$
\left(\begin{array}{llllllllll}
1 & 0 & 1 & 1 & 1 & 0 & 1 & 1 & 0 & 2 \\
0 & 1 & 1 & 2 & 3 & 0 & 1 & 3 & 0 & 1 \\
0 & 0 & 4 & 4 & 0 & 1 & 0 & 4 & 0 & 0 \\
0 & 0 & 0 & 0 & 4 & 4 & 0 & 0 & 1 & 0
\end{array}\right)
$$

Matroid number 1. $M_{10,1}$ has path sequence ending in the triangle $\{6,8,9\}$. $\mathrm{GF}(5)$-representation (first coordinate):

$$
\left(\begin{array}{llllllllll}
1 & 0 & 1 & 1 & 1 & 0 & 1 & 1 & 0 & 1 \\
0 & 1 & 1 & 2 & 3 & 0 & 1 & 3 & 0 & 1 \\
0 & 0 & 4 & 4 & 0 & 1 & 0 & 4 & 0 & 0 \\
0 & 0 & 0 & 0 & 4 & 4 & 0 & 0 & 1 & 4
\end{array}\right)
$$


Matroid number 2. $M_{10,2}$ has a path sequence ending in the 4-point segment $\{0,1,6,9\} \cdot \operatorname{GF}(5)$-representation (first coordinate):

$$
\left(\begin{array}{llllllllll}
1 & 0 & 1 & 1 & 1 & 0 & 1 & 1 & 0 & 3 \\
0 & 1 & 1 & 2 & 3 & 0 & 1 & 3 & 0 & 3 \\
0 & 0 & 4 & 4 & 0 & 1 & 0 & 4 & 0 & 0 \\
0 & 0 & 0 & 0 & 4 & 4 & 0 & 0 & 1 & 4
\end{array}\right)
$$

Matroid number 13. $M_{10,13}$ has path sequence ending with fan $(2,3,7,8,9)$. $\mathrm{GF}(5)$-representation (first coordinate):

$$
\left(\begin{array}{llllllllll}
1 & 0 & 1 & 1 & 1 & 0 & 1 & 1 & 0 & 1 \\
0 & 1 & 1 & 2 & 3 & 0 & 1 & 3 & 0 & 3 \\
0 & 0 & 4 & 4 & 0 & 1 & 0 & 4 & 0 & 4 \\
0 & 0 & 1 & 3 & 0 & 4 & 0 & 0 & 1 & 1
\end{array}\right)
$$

Matroid number 14. $M_{10,14}$ has a path sequence ending with 4-segment $\{0,1,4,6\}$. GF(5)-representation (first coordinate):

$$
\left(\begin{array}{llllllllll}
1 & 0 & 1 & 1 & 1 & 0 & 1 & 1 & 0 & 0 \\
0 & 1 & 1 & 2 & 3 & 0 & 1 & 3 & 0 & 1 \\
0 & 0 & 4 & 4 & 0 & 1 & 0 & 4 & 0 & 0 \\
0 & 0 & 1 & 3 & 0 & 4 & 0 & 0 & 1 & 2
\end{array}\right)
$$

Matroid number 21. $M_{10,21}$ has a path sequence ending in the triangle $\{1,8,9\}$. $\mathrm{GF}(5)$-representation (first coordinate):

$$
\left(\begin{array}{llllllllll}
1 & 0 & 1 & 1 & 1 & 0 & 1 & 1 & 0 & 0 \\
0 & 1 & 1 & 2 & 3 & 0 & 1 & 3 & 0 & 4 \\
0 & 0 & 4 & 4 & 0 & 1 & 0 & 4 & 0 & 0 \\
0 & 0 & 1 & 3 & 4 & 4 & 0 & 0 & 1 & 2
\end{array}\right)
$$


Matroid number 3. $M_{10,3}$ has path sequence ending in the fan $(9,1,8,7)$. $\mathrm{GF}(5)$-representation (first coordinate):

$$
\left(\begin{array}{llllllllll}
1 & 0 & 1 & 1 & 1 & 0 & 1 & 0 & 0 & 4 \\
0 & 1 & 1 & 2 & 3 & 0 & 1 & 0 & 0 & 2 \\
0 & 0 & 4 & 4 & 0 & 1 & 0 & 0 & 0 & 0 \\
3 & 0 & 0 & 4 & 4 & 0 & 0 & 1 & 0 & 1 \\
0 & 0 & 0 & 0 & 2 & 4 & 4 & 0 & 1 & 0
\end{array}\right)
$$

Matroid number 4. $M_{10,4}$ has a path sequence ending in the fan $(9,1,8,3)$. $\mathrm{GF}(5)$-representation (first coordinate):

$$
\left(\begin{array}{llllllllll}
1 & 0 & 1 & 1 & 1 & 0 & 1 & 0 & 0 & 0 \\
0 & 1 & 1 & 2 & 3 & 0 & 1 & 0 & 0 & 2 \\
0 & 0 & 4 & 4 & 0 & 1 & 0 & 0 & 0 & 0 \\
3 & 0 & 0 & 4 & 4 & 0 & 0 & 1 & 0 & 4 \\
0 & 0 & 0 & 0 & 2 & 4 & 4 & 0 & 1 & 0
\end{array}\right)
$$

Matroid number 5. $M_{10,5}$ has a path sequence ending with fan $(8,4,0,6)$. GF(5)-representation (first coordinate):

$$
\left(\begin{array}{llllllllll}
1 & 0 & 1 & 1 & 1 & 0 & 1 & 0 & 0 & 0 \\
0 & 1 & 1 & 2 & 3 & 0 & 1 & 0 & 0 & 1 \\
0 & 0 & 4 & 4 & 0 & 1 & 0 & 0 & 0 & 0 \\
3 & 0 & 0 & 4 & 4 & 0 & 0 & 1 & 0 & 4 \\
0 & 0 & 0 & 0 & 2 & 4 & 4 & 0 & 1 & 0
\end{array}\right)
$$

Matroid number 6. $M_{10,6}$ has a path sequence ending with the fan $(6,0,4,8,9)$. $\mathrm{GF}(5)$-representation (first coordinate):

$$
\left(\begin{array}{llllllllll}
1 & 0 & 1 & 1 & 1 & 0 & 1 & 0 & 0 & 1 \\
0 & 1 & 1 & 2 & 3 & 0 & 1 & 0 & 0 & 3 \\
0 & 0 & 4 & 4 & 0 & 1 & 0 & 0 & 0 & 4 \\
3 & 0 & 0 & 4 & 4 & 0 & 0 & 1 & 0 & 4 \\
0 & 0 & 0 & 0 & 2 & 4 & 4 & 0 & 1 & 0
\end{array}\right)
$$


Matroid number 7. $M_{10,7}$ has a path sequence ending in the triangle $\{4,8,9\}$. $\mathrm{GF}(5)$-representation (first coordinate):

$$
\left(\begin{array}{llllllllll}
1 & 0 & 1 & 1 & 1 & 0 & 1 & 0 & 0 & 1 \\
0 & 1 & 1 & 2 & 3 & 0 & 1 & 0 & 0 & 1 \\
0 & 0 & 4 & 4 & 0 & 1 & 0 & 0 & 0 & 4 \\
3 & 0 & 0 & 4 & 4 & 0 & 0 & 1 & 0 & 4 \\
0 & 0 & 0 & 0 & 2 & 4 & 4 & 0 & 1 & 0
\end{array}\right)
$$

Matroid number 8. $M_{10,8}$ has a path sequence ending in the fan $(3,7,1,9)$. $\mathrm{GF}(5)$-representation (first coordinate):

$$
\left(\begin{array}{llllllllll}
1 & 0 & 1 & 1 & 1 & 0 & 1 & 0 & 0 & 3 \\
0 & 1 & 1 & 2 & 3 & 0 & 1 & 0 & 0 & 4 \\
0 & 0 & 4 & 4 & 0 & 1 & 0 & 0 & 0 & 0 \\
3 & 0 & 0 & 4 & 4 & 0 & 0 & 1 & 0 & 2 \\
0 & 0 & 0 & 0 & 2 & 4 & 4 & 0 & 1 & 2
\end{array}\right)
$$

Matroid number 9. $M_{10,9}$ has a path sequence ending in the 4-point segment $\{0,4,6,9\} \cdot \operatorname{GF}(5)$-representation (first coordinate):

$$
\left(\begin{array}{llllllllll}
1 & 0 & 1 & 1 & 1 & 0 & 1 & 0 & 0 & 0 \\
0 & 1 & 1 & 2 & 3 & 0 & 1 & 0 & 0 & 3 \\
0 & 0 & 4 & 4 & 0 & 1 & 0 & 0 & 0 & 0 \\
3 & 0 & 0 & 4 & 4 & 0 & 0 & 1 & 0 & 0 \\
0 & 0 & 0 & 0 & 2 & 4 & 4 & 0 & 1 & 2
\end{array}\right)
$$

Matroid number 10. $M_{10,10}$ has a path sequence ending in the fan $(9,1,3,8)$. GF(5)-representation (first coordinate):

$$
\left(\begin{array}{llllllllll}
1 & 0 & 1 & 1 & 1 & 0 & 1 & 0 & 0 & 0 \\
0 & 1 & 1 & 2 & 3 & 0 & 1 & 0 & 0 & 4 \\
0 & 0 & 4 & 4 & 0 & 1 & 0 & 0 & 0 & 0 \\
3 & 0 & 0 & 4 & 4 & 0 & 0 & 1 & 0 & 0 \\
0 & 0 & 0 & 0 & 2 & 4 & 4 & 0 & 1 & 2
\end{array}\right)
$$


Matroid number 11. $M_{10,11}$ has a path sequence ending in fan $(0,4,6,8)$. $\mathrm{GF}(5)$-representation (first coordinate):

$$
\left(\begin{array}{llllllllll}
1 & 0 & 1 & 1 & 1 & 0 & 1 & 0 & 0 & 4 \\
0 & 1 & 1 & 2 & 3 & 0 & 1 & 0 & 0 & 2 \\
0 & 0 & 4 & 4 & 0 & 1 & 0 & 0 & 0 & 1 \\
3 & 0 & 0 & 4 & 4 & 0 & 0 & 1 & 0 & 1 \\
0 & 0 & 0 & 0 & 2 & 4 & 4 & 0 & 1 & 2
\end{array}\right)
$$

Matroid number 12. $M_{10,12}$ has a path sequence ending in the 4-point coline $\{1,3,7,8\} \cdot G F(5)$-representation (first coordinate):

$$
\left(\begin{array}{llllllllll}
1 & 0 & 1 & 1 & 1 & 0 & 1 & 0 & 0 & 0 \\
0 & 1 & 1 & 2 & 3 & 0 & 1 & 0 & 0 & 3 \\
0 & 0 & 4 & 4 & 0 & 1 & 0 & 0 & 0 & 0 \\
3 & 0 & 0 & 4 & 4 & 0 & 0 & 1 & 0 & 1 \\
0 & 0 & 0 & 0 & 2 & 4 & 4 & 0 & 1 & 2
\end{array}\right)
$$

Matroid number 18. $\quad M_{10,18}$ has a path sequence ending in the fan $(4,0,1,7,9)$. $\mathrm{GF}(5)$-representation (first coordinate):

$$
\left(\begin{array}{llllllllll}
1 & 0 & 1 & 1 & 1 & 0 & 1 & 0 & 0 & 1 \\
0 & 1 & 1 & 2 & 3 & 0 & 1 & 0 & 0 & 1 \\
0 & 0 & 4 & 4 & 0 & 1 & 0 & 0 & 0 & 4 \\
4 & 0 & 0 & 2 & 4 & 0 & 0 & 1 & 0 & 2 \\
0 & 0 & 0 & 3 & 0 & 4 & 0 & 0 & 1 & 3
\end{array}\right)
$$

Matroid number 15. $M_{10,15}$ has a path sequence ending in the fan $(4,0,1,7)$. $\mathrm{GF}(5)$-representation (first coordinate):

$$
\left(\begin{array}{llllllllll}
1 & 0 & 1 & 1 & 1 & 0 & 1 & 0 & 0 & 3 \\
0 & 1 & 1 & 2 & 3 & 0 & 1 & 0 & 0 & 4 \\
0 & 0 & 4 & 4 & 0 & 1 & 0 & 0 & 0 & 2 \\
4 & 0 & 0 & 2 & 4 & 0 & 0 & 1 & 0 & 2 \\
0 & 0 & 0 & 3 & 0 & 4 & 0 & 0 & 1 & 3
\end{array}\right)
$$


Matroid number 16. $M_{10,16}$ has a path sequence ending in the fan $(4,0,1,7)$. GF(5)-representation (first coordinate):

$$
\left(\begin{array}{llllllllll}
1 & 0 & 1 & 1 & 1 & 0 & 1 & 0 & 0 & 0 \\
0 & 1 & 1 & 2 & 3 & 0 & 1 & 0 & 0 & 1 \\
0 & 0 & 4 & 4 & 0 & 1 & 0 & 0 & 0 & 0 \\
4 & 0 & 0 & 2 & 4 & 0 & 0 & 1 & 0 & 2 \\
0 & 0 & 0 & 3 & 0 & 4 & 0 & 0 & 1 & 3
\end{array}\right)
$$

Matroid number 19. $M_{10,19}$ has a path sequence ending in the fan $(4,0,1,7)$. GF(5)-representation (first coordinate):

$$
\left(\begin{array}{llllllllll}
1 & 0 & 1 & 1 & 1 & 0 & 1 & 0 & 0 & 2 \\
0 & 1 & 1 & 2 & 3 & 0 & 1 & 0 & 0 & 2 \\
0 & 0 & 4 & 4 & 0 & 1 & 0 & 0 & 0 & 3 \\
4 & 0 & 0 & 2 & 4 & 0 & 0 & 1 & 0 & 0 \\
0 & 0 & 0 & 3 & 0 & 4 & 0 & 0 & 1 & 1
\end{array}\right)
$$

Matroid number 20. $M_{10,20}$ has a path sequence ending in the triangle $\{0,1,4\}$. $\mathrm{GF}(5)$-representation (first coordinate):

$$
\left(\begin{array}{llllllllll}
1 & 0 & 1 & 1 & 1 & 0 & 1 & 0 & 0 & 0 \\
0 & 1 & 1 & 2 & 3 & 0 & 1 & 0 & 0 & 3 \\
0 & 0 & 4 & 4 & 0 & 1 & 0 & 0 & 0 & 0 \\
4 & 0 & 0 & 2 & 4 & 0 & 0 & 1 & 0 & 1 \\
0 & 0 & 0 & 3 & 0 & 4 & 0 & 0 & 1 & 0
\end{array}\right)
$$




\section{Chapter 4}

\section{On excluded minors for $\mathbb{H}_{5}$ - or $\mathbb{U}_{2}$-representable matroids}

We prove that if a matroid, $M$, is an excluded minor for the class of $\mathbb{H}_{5^{-}}$ representable matroids or the class of $\mathbb{U}_{2}$-representable matroids satisfying a certain "robustness" condition, and $M$ has a pair of elements $a, b$ such that $M \backslash a, b$ is 3 -connected with a $\left\{U_{2,5}, U_{3,5}\right\}$-minor, then there is a bound on the size of $|E(M)|$. In particular, this implies that there are finitely many such excluded minors.

\subsection{Introduction}

Let $M$ be a 3-connected matroid. We say that $M$ has a 3-connected deletion pair keeping a $\left\{U_{2,5}, U_{3,5}\right\}$-minor if there is some pair of elements $a, b$ of $M$ such that $M \backslash a, b$ is 3 -connected and $M \backslash a, b$ has a $\left\{U_{2,5}, U_{3,5}\right\}$-minor.

Let $M$ be an excluded minor for the class of $\mathbb{H}_{5}$-representable matroids or the class of $\mathbb{U}_{2}$-representable matroids with a 3-connected deletion pair keeping a $\left\{U_{2,5}, U_{3,5}\right\}$-minor. By Theorem 1.0.1, if $M$ is sufficiently large, then, up to replacing $M$ by an excluded minor that is $\Delta-\nabla$-equivalent to $M$ or $M^{*}$, we can assume that $M$ is $U_{2,5^{-}}$or $U_{3,5}$-fragile. Now, if $M \backslash a, b$ is $\left\{U_{2,5}, U_{3,5}\right\}$-fragile, then can use our knowledge of the structure of $M \backslash a, b$, Theorem 1.0.2, to try to bound the size of $M$. Thus we say that $M$ is a robust excluded minor if, up to replacing $M$ by an excluded minor that is $\Delta-\nabla$-equivalent to $M$ or $M^{*}$, there is no 3-connected deletion pair keeping a $\left\{U_{2,5}, U_{3,5}\right\}$-minor, $a^{\prime}, b^{\prime}$, such that $M \backslash a^{\prime}, b^{\prime}$ is 
$U_{2,5^{-}}$or $U_{3,5}$-fragile but not $\left\{U_{2,5}, U_{3,5}\right\}$-fragile.

The main goal of this chapter is to prove the following result.

Theorem 4.1.1. There are finitely many robust excluded minors for the class of $\mathbb{H}_{5}$ - or $\mathbb{U}_{2}$-representable matroids that have a 3-connected deletion pair keeping a $\left\{U_{2,5}, U_{3,5}\right\}$-minor.

Our proof of Theorem 4.1.1 crucially relies on the main Theorems of Chapter 2 and Chapter 3 to construct a bound on the size of the ground set of a robust excluded minor $M$ with a 3 -connected deletion pair keeping a $\left\{U_{2,5}, U_{3,5}\right\}$-minor.

We note that although the bound achieved here is not sufficiently small for us to feasibly search for all such excluded minors, we believe that a feasible bound could be obtained by refining these techniques and employing a more careful analysis. In particular, the outcomes stated in Theorem 2.1.1(b)(ii) and Theorem 2.1 .2 (b) are obstacles to a smaller bound on $|E(M)|$, and refining these outcomes is a priority for future research.

Another goal for future research is to bound the size of the non-robust excluded minors that have a 3 -connected deletion pair keeping a $\left\{U_{2,5}, U_{3,5}\right\}$-minor. One possible approach would be to upgrade Theorem 1.0.1 using sets of stabilizers rather than a fixed stabilizer, which would be desirable for future applications. Another approach would be to characterise the structure of the $U_{2,5^{-}}$or $U_{3,5^{-}}$ fragile matroids that are not $\left\{U_{2,5}, U_{3,5}\right\}$-fragile. The following proposition leads us to believe that the structure of the $U_{2,5^{-}}$or $U_{3,5^{-}}$fragile matroids that are not $\left\{U_{2,5}, U_{3,5}\right\}$-fragile would be relatively simple to characterise.

Proposition 4.1.2. [20] Proposition 12.2.15] If $M$ is 3-connected with rank and corank at least three, then $M$ has a $U_{2,5}$-minor if and only if $M$ has a $U_{3,5}$-minor.

The chapter is structured as follows. After stating necessary preliminaries, we look at the structural consequences of having a robust basis in Section 4.3 . The robust basis together with our knowledge of the structure of $\left\{U_{2,5}, U_{3,5}\right\}$-fragile matroids enables us to quickly reduce to the case where $M \backslash a, b$ has a path of 3separations. In Section 4.4 we show that an end triad in the path of 3-separations of $M \backslash a, b$ is blocked in $M$. We can think of this blocked triad as our certificate of non-fragility. We then look for new deletion and contraction pairs in Section 4.5 that preserve the blocked triad. Finally, in Section 4.6, we obtain the bound of the size of $|E(M)|$. 


\subsection{Preliminaries}

We will frequently appeal to orthogonality, sometimes in the following form.

Lemma 4.2.1. Let $(X,\{e\}, Y)$ be a partition of $E(M)$. Then $e \in \operatorname{cl}(X)$ if and only if $e \notin \mathrm{cl}^{*}(Y)$.

The following fact will be used when we consider the structure of circuits and cocircuits of $M^{\prime}$ relative to a path of 3-separations of $M$.

Lemma 4.2.2. Let $(X, Y)$ be an exactly 3-separating partition of a 3-connected matroid $M$. Suppose $|X| \geq 3$ and $x \in X$. Then

(i) $x \in \mathrm{cl}^{(*)}(X-\{x\})$; and

(ii) $(X-\{x\}, Y \cup\{x\})$ is exactly 3-separating if and only if $x$ is in exactly one of $\operatorname{cl}(X-\{x\}) \cap \operatorname{cl}(Y)$ and $\mathrm{cl}^{*}(X-\{x\}) \cap \mathrm{cl}^{*}(Y)$.

We use the fact that fragile matroids are 3-connected up to series and parallel classes.

Proposition 4.2.3. [19] Proposition 4.4] Let $M$ be a strictly $\left\{U_{2,5}, U_{3,5}\right\}$-matroid. If $(X, Y)$ is a 2-separation of $M$, then $X$ or $Y$ is either a series or parallel class.

We use the description of the structure of fragile matroids, which we state again here for convenience.

Theorem 4.2.4. [2. Theorem 1.3] Let $M^{\prime}$ be a 3-connected strictly $\left\{U_{2,5}, U_{3,5}\right\}$ fragile $\mathbb{H}_{5}$-representable matroid. Then $M^{\prime}$ is isomorphic to a matroid $M$ for which one of the following holds:

(i) $M$ has an $\left\{X_{8}, Y_{8}, Y_{8}^{*}\right\}$-minor;

(ii) $M \in\left\{U_{2,6}, U_{4,6}, P_{6}, M_{9,9}, M_{9,9}^{*}\right\}$;

(iii) $M$ or $M^{*}$ can be obtained from $U_{2,5}$ (with groundset $\{a, b, c, d, e\}$ ) by gluing wheels to $(a, c, b),(a, d, b),(a, e, b)$;

(iv) $M$ or $M^{*}$ can be obtained from $U_{2,5}$ (with groundset $\{a, b, c, d, e\}$ ) by gluing wheels to $(a, b, c),(c, d, e)$; 
(v) $M$ or $M^{*}$ can be obtained from $M_{7,1}$ by gluing a wheel to $(1,3,2)$.

For fragile matroids with an $\left\{X_{8}, Y_{8}, Y_{8}^{*}\right\}$-minor, we recall the following constructive description.

Theorem 4.2.5. If $M$ is a 3-connected $\left\{U_{2,5}, U_{3,5}\right\}$-fragile $\mathbb{H}_{5}$-representable matroid that has an $\left\{X_{8}, Y_{8}, Y_{8}^{*}\right\}$-minor, then there is some path sequence that obtains $M$.

In particular, we will make use of the following path of 3-separations.

Proposition 4.2.6. Let $\mathbb{P} \in\left\{\mathbb{U}_{2}, \mathbb{H}_{5}\right\}$. If $M$ is a $\left\{U_{2,5}, U_{3,5}\right\}$-fragile $\mathbb{H}_{5}$ representable matroid that has an $\left\{X_{8}, Y_{8}, Y_{8}^{*}\right\}$-minor, then $M$ has a path of 3separations $\boldsymbol{P}=\left(P_{1}, \ldots, P_{n}\right)$ such that the end steps $P_{1}$ and $P_{n}$ are path-generating sets, and, for each internal step $P_{i}$ of $\boldsymbol{P},\left|P_{i}\right| \leq 3$ and $P_{i}$ is alternately the guts and coguts of the 3-separation $\left(P_{1} \cup \cdots \cup P_{i}, P_{i+1} \cup \cdots \cup P_{n}\right)$.

We will assume that $A$ is a companion matrix for $M$. That is, $A$ is an $B \times B^{*}$ matrix with entries in $\mathbb{P} \in\left\{\mathbb{H}_{5}, \mathbb{U}_{2}\right\}$ such that $B$ is a basis of $M$ and $a, b \in B^{*}$. Assume that $A-a$ and $A-b$ are $\mathbb{P}$-matrices such that $M \backslash a=M[I \mid(A-a)]$ and $M \backslash b=M[I \mid(A-b)]$. We can also assume that there are elements $x, y \in B$ such that $\{a, b, x, y\}$ incriminates $(M, A)$.

If $p \in B$ and $q \in B^{*}-\{a, b\}$ are elements such that $A_{p q} \neq 0$, then a pivot on $A_{p q}$ is allowable if $p \in\{x, y\}$ or $A_{p a}=A_{p b}=0$ or $A_{x q}=A_{y q}=0$. The following results show that allowable pivots preserve the property of having a companion matrix for $M$ with a 4-element incriminating set.

Lemma 4.2.7. [19 Lemma 5.10] If $p \in\{x, y\}, q \in B^{*}-\{a, b\}$, and $A_{p q} \neq 0$, then $\{x, y, a, b\} \triangle\{p, q\}$ incriminates $\left(M, A^{p q}\right)$.

If the $x$ and $y$ entries are both zero or the $a$ and $b$ entries are both zero, then we can pivot without changing the incriminating set.

Lemma 4.2.8. [19] Lemma 5.11] If $p \in B-\{x, y\}, q \in B^{*}-\{a, b\}$ are such that $A_{p q} \neq 0$, and either $A_{p a}=A_{p b}=0$ or $A_{x q}=A_{y q}=0$, then $\{a, b, x, y\}$ incriminates $\left(M, A^{p q}\right)$.

We also use the fact that the entries of $A$ labelled by members of the incriminating set $\{a, b, x, y\}$ are non-zero. 
Lemma 4.2.9. If $\{a, b, x, y\}$ is an incriminating set for the pair $(M, A)$, then $A_{i j} \neq$ 0 for $i \in\{x, y\}$ and $j \in\{a, b\}$.

Let $M$ be a 3-connected matroid, let $B$ be a basis of $M$, and let $N$ be a 3-connected minor of $M$. Recall that an element $e$ of $M$ is $(N, B)$-robust if either

(i) $e \in B$ and $M / e$ has an $N$-minor; or

(ii) $e \in E-B$ and $M \backslash e$ has an $N$-minor.

An element $e$ of $M$ is $(N, B)$-strong if either

(i) $e \in B$, and $\operatorname{si}(M / e)$ is 3-connected and has an $N$-minor; or

(ii) $e \in E-B$, and $\operatorname{co}(M \backslash e)$ is 3-connected and has an $N$-minor.

Recall that a basis $B$ of $M$ is a robust basis for $M$ if, for any basis $B^{\prime}$ of $M$, the number of $\left(N, B^{\prime}\right)$-robust elements of $M \backslash a, b$ outside of $\left\{x^{\prime}, y^{\prime}\right\}$ is at most the number of $(N, B)$-robust elements of $M \backslash a, b$ outside of $\{x, y\}$. When $M$ has some basis $B$ that has an $(N, B)$-strong element outside of $\{x, y\}$, the maximum number of $(N, B)$-robust elements outside of $\{x, y\}$ in a robust basis is subject to the constraint that $B$ displays a triad of the form $\{x, y, z\}$, where $z$ is an $(N, B)$-strong element of $M \backslash a, b$.

In particular, if $B$ is a robust basis, and a pivot on an entry $A_{p q} \neq 0$ of the companion matrix $A$ for $M$ is allowable, then it follows that $M$ cannot have more $(N, B \triangle\{p, q\})$-robust elements than $(N, B)$-robust elements.

We can now recall the connection between excluded minors and fragile matroids, which is contained in the next two Theorems.

Theorem 4.2.10. Let $M$ be an excluded minor for the class of $\mathbb{P}$-representable matroids, and let $N$ be a strong $\mathbb{P}$-stabilizer. If $M$ has a pair of elements $a, b$ such that $M \backslash a, b$ is 3-connected with an $N$-minor, then at least one of the following holds:

(a) $|E(M)| \leq|E(N)|+16$ or $r(M) \leq r(N)+5$; or

(b) there is some robust basis B for M such that either: 
(i) $M \backslash a, b$ is $N$-fragile, and $M \backslash a, b$ has at most one $(N, B)$-robust element $z \in B^{*}-\{a, b\}$ outside of $\{x, y\}$. Moreover, if $z \in B^{*}-\{a, b\}$ is $(N, B)$ robust, then $z$ is an $(N, B)$-strong element of $M \backslash a, b$, and $\{x, y, z\}$ is a triad of $M \backslash a, b$; or

(ii) $M \backslash a, b$ is not $N$-fragile, but the only $N$-flexible elements of $M \backslash a, b$ are contained in a triad $\{x, y, z\}$ of $M \backslash a, b$ for some $(N, B)$-strong $z \in B^{*}$. Moreover, the only $(N, B)$-robust elements of $M \backslash a, b$ are in $\{x, y, z\}$, and $M$ has a 4-element cocircuit $\{p, x, y, z\}$ or 5-element cocircuit $\{a, b, x, y, z\}$ that contains a triangle $\{p, x, y\}$ for some $p \in\{a, b\}$.

Recall that a matroid $M^{\prime}$ is $\Delta-\nabla$-equivalent to $M$ if there is some sequence of matroids $M_{1}, \ldots, M_{n}$ such that $M=M_{1}, M^{\prime}=M_{n}$, and, for each $i \in\{2, \ldots, n\}$, there is some segment or cosegment $A$ of $M_{i-1}$ such that $M_{i}=\nabla_{A}\left(M_{i-1}\right)$ or $M_{i}=\Delta_{A}\left(M_{i-1}\right)$. Oxley, Semple, and Vertigan [21, Theorem 1.1] proved that excluded minors for the class of $\mathbb{P}$-representable matroids are closed under $\Delta$ $\nabla$-equivalence.

Theorem 4.2.11. Let $M$ be an excluded minor for the class of $\mathbb{P}$-representable matroids, and let $N$ be a strong $\mathbb{P}$-stabilizer. If $M$ has a pair of elements $a, b$ such that $M \backslash a, b$ is 3-connected with an $N$-minor, then there is some excluded minor $M^{\prime}$ that is $\Delta$ - $\nabla$-equivalent to $M^{\prime \prime} \in\left\{M, M^{*}\right\}$ with a minor $N^{\prime} \in\left\{N, N^{*}\right\}$, a pair of elements $a^{\prime}, b^{\prime}$ such that $M^{\prime} \backslash a^{\prime}, b^{\prime}$ is 3-connected with an $N^{\prime}$-minor such that at least one of the following holds:

(a) $\left|E\left(M^{\prime}\right)\right| \leq\left|E\left(N^{\prime}\right)\right|+16$; or

(b) $\min \left\{r\left(M^{\prime}\right), r^{*}\left(M^{\prime}\right)\right\} \leq \max \left\{r\left(N^{\prime}\right), r^{*}\left(N^{\prime}\right)\right\}+7$; or

(c) there is some robust basis $B^{\prime}$ for $M^{\prime}$ such that $M^{\prime} \backslash a^{\prime}, b^{\prime}$ is $N^{\prime}$-fragile, and $M^{\prime} \backslash a^{\prime}, b^{\prime}$ has at most one $\left(N^{\prime}, B^{\prime}\right)$-robust element $z \in\left(B^{\prime}\right)^{*}-\left\{a^{\prime}, b^{\prime}\right\}$ outside of $\left\{x^{\prime}, y^{\prime}\right\}$. Moreover, if $z \in\left(B^{\prime}\right)^{*}-\left\{a^{\prime}, b^{\prime}\right\}$ is an $\left(N^{\prime}, B^{\prime}\right)$-robust element, then $z$ is an $\left(N^{\prime}, B^{\prime}\right)$-strong element of $M^{\prime} \backslash a^{\prime}, b^{\prime}$, and $\left\{x^{\prime}, y^{\prime}, z\right\}$ is a triad of $M^{\prime} \backslash a^{\prime}, b^{\prime}$.

Recall that a subset $G$ of $M^{\prime}$ is a gadget if $\{x, y\} \subseteq G$, there are triads $T$ and $T^{\prime}$ of $M^{\prime}$ such that $G=T \cup T,\left|T \cap T^{\prime}\right| \in\{1,2\}$, and $G \subseteq \operatorname{cl}^{*}\left(G \cap B^{*}\right)$. If $\left|T \cap T^{\prime}\right|=1$, then at least one $(N, B)$-strong deletable element in $G$. A gadget is a structure that enables us to bound the size of $M$. 
Lemma 4.2.12. If $M^{\prime}$ has a gadget, then $|E(M)| \leq|E(N)|+16$.

We use the following results to show that certain deletion pairs commute with the $\Delta$ - $\nabla$-exchange operation.

Lemma 4.2.13. [21] Corollary 2.17] Suppose that $x \in E(M)-A$ and $|A| \geq 3$. Suppose that $\nabla_{A}(M)$ is defined. If $M \backslash x$ is 3-connected, then $\nabla_{A}(M \backslash x)$ is defined and $\nabla_{A}(M \backslash x)=\nabla_{A}(M) \backslash x$.

Lemma 4.2.14. Let $M$ be a 3-connected matroid, and let $T$ be an independent cosegment of $M$ with $|T| \geq 3$. If $M$ has a pair of elements $a, b \in E(M)-T$ such that $M \backslash a, b$ is 3-connected, $\nabla_{T}(M \backslash a, b)$ is defined, and $\nabla_{T}(M \backslash a, b)$ is 3-connected, then $\nabla_{T}(M) \backslash a, b$ is 3-connected and $\nabla_{T}(M \backslash a, b)=\nabla_{T}(M) \backslash a, b$.

Proof. Since $\nabla_{T}(M)$ is defined and $M \backslash a$ is 3-connected, it follows from Lemma 4.2 .13 that $\nabla_{T}(M \backslash a)$ is defined and $\nabla_{T}(M \backslash a)=\nabla_{T}(M) \backslash a$. Now $\nabla_{T}(M \backslash a)$ is defined and $M \backslash a, b$ is 3-connected, so it follows again from Lemma 4.2.13 that $\nabla_{T}(M \backslash a, b)$ is defined and $\nabla_{T}(M \backslash a, b)=\nabla_{T}(M \backslash a) \backslash b$. Hence $\nabla_{T}(M \backslash a, b)$ is defined and $\nabla_{T}(M \backslash a, b)=\nabla_{T}(M) \backslash a, b$.

We use the following result in the case of $\mathrm{GF}(5)$-representable matroids in our final bound.

Lemma 4.2.15. [20. Corollary 6.1.7] A 3-connected rank-r $\mathrm{GF}(q)$-representable matroid has at most $\left(q^{r}-1\right) /(q-1)$ elements.

\subsection{Robust basis wins}

In this section, we suppose that there is some robust basis $B$ for $M$ such that $M \backslash a, b$ is $N$-fragile, and $M \backslash a, b$ has at most one $(N, B)$-robust element $z \in B^{*}-\{a, b\}$ outside of $\{x, y\}$. Moreover, if $z \in B^{*}-\{a, b\}$ is $(N, B)$-robust, then $z$ is an $(N, B)$ strong element of $M \backslash a, b$, and $\{x, y, z\}$ is a triad of $M \backslash a, b$. Recall that we assume that $x, y \in B$ belong to a 4-element set $\{a, b, x, y\}$ that incriminates $(M, A)$.

We now exploit some of the structure this basis imposes on $M^{\prime}=M \backslash a, b$. Of particular importance, we are able to bound the size of fans in $M^{\prime}$.

Lemma 4.3.1. The elements $x$ and $y$ are not deletable in $M \backslash a, b$. 
Proof. Supose that $M^{\prime}$ has an $(N, B)$-robust element $z \in B^{*}-\{a, b\}$. Then since $\{x, y, z\}$ is a triad of $M^{\prime}$ and $z$ is deletable, it follows that $x$ and $y$ are contractible. But $M^{\prime}$ is fragile, so $x$ and $y$ are non deletable. Supose that $M^{\prime}$ has no $(N, B)$-robust elements outside of $\{x, y\}$. Then $x$ and $y$ are not deletable because $B$ is a robust basis.

Lemma 4.3.2. If $M \backslash a, b$ has a triangle $T$, then $T$ is allowable.

Proof. Suppose that $M^{\prime}$ has a triangle $T$ such that all of the elements of $T$ are deletable. Then $T$ cannot be a subset of the basis $X$, so $B^{*}-\{a, b\}$ must contain a deletable element of $T$. Then there is some $(N, B)$-element $z$ of $M^{\prime}$ outside of $\{x, y\}$, and $z \in T$. But $\{x, y, z\}$ is a triad of $M$, so by orthogonality $T \cap\{x, y\}$ is non-empty; a contradiction because $x$ and $y$ are not deletable by Lemma 4.3.1.

Corollary 4.3.3. If $M^{\prime}$ has a 4-segment $S$, then $S$ has at most two deletable elements.

Lemma 4.3.4. $M^{\prime}$ has at most one triangle $T$ such that $T$ contains two deletable elements. Moreover, if $T$ exists, then $M^{\prime}$ has an allowable triad $\{x, y, z\}$ and $T \cup$ $\{x, y\}$ is a 4-element fan of $M^{\prime}$.

Proof. Assume that $T=\{p, q, r\}$ is a triangle of $M^{\prime}$, where $p, q$ are deletable and $r$ is not deletable. Suppose that $\{p, q\} \subseteq B-\{x, y\}$. Then $r \in B^{*}-\{a, b\}$ because $T$ is not contained in the basis $B$. But then $A_{x r}=A_{y r}=0$, so a pivot on $A_{p r} \neq 0$ is allowable, and $A^{p r}$ has more $\left(N, B^{\prime}\right)$-robust elements; a contradiction because $B$ is a robust basis. We may therefore assume that $p \in B^{*}-\{a, b\}$. Then $p$ is an $(N, B)$-robust element outside of $\{x, y\}$, so $\{p, x, y\}$ is a triad. By orthogonality with $T$ and 4.3.1, it follows that $r \in\{x, y\}$. Assume that $x=r$. Then $T \cup\{x, y\}$ is a 4-element fan with ordering $(s, p, r, y)$.

Now, suppose that $T^{\prime}$ is another triangle with two deletable elements. Then by the same argument $T^{\prime}$ contains $p$ and $y$. But $T \cup T^{\prime}$ has rank three and $T \cup\{x, y\}$ already contains three members of $B$, so there is some deletable element of $T^{\prime}$ in $B^{*}-\{p\}$; a contradiction because the only $(N, B)$-robust elements of $M^{\prime}$ are in $\{p, x, y\}$.

Lemma 4.3.5. If $M^{\prime}$ or $\left(M^{\prime}\right)^{*}$ is obtained from a fragile matroid $M^{\prime \prime}$ by gluing a wheel to a triangle of $M^{\prime \prime}$, then the corresponding fan $F$ of $M^{\prime}$ has exactly one triangle and $|F| \leq 5$. 
Proof. Let $F$ be the corresponding fan of $M^{\prime}$. Suppose that $|F| \geq 5$. Then every rim element of $F$ is contractible, and every spoke element of $F$ is deletable. Now suppose that $|F| \geq 6$. Then $M^{\prime}$ has two triangles contained in $F$ that each contain at least two deletable elements; a contradiction of Lemma 4.3.4. Thus $|F| \leq 5$, and if $|F|=5$, then $F$ must have rim ends.

These simple results enable us to bound the robust excluded minors such that $M \backslash a, b$ has no $\left\{X_{8}, Y_{8}, Y_{8}^{*}\right\}$-minor. Note that this bound is unlikely to be sharp, and should be relatively easy to improve in future research.

Lemma 4.3.6. If $M^{\prime}$ has no $\left\{X_{8}, Y_{8}, Y_{8}^{*}\right\}$-minor, then $|E(M)| \leq 17$.

Proof. Suppose that $M \backslash a, b \in\left\{U_{2,6}, U_{4,6}, P_{6}, Q_{6}, M_{9,9}, M_{9,9}^{*}\right\}$. Then clearly $|E(M)| \leq 11$.

Suppose that $M^{\prime}$ or $\left(M^{\prime}\right)^{*}$ is obtained from $M_{7,1}$ by gluing a wheel to a triangle, then the corresponding fan $F$ of $M^{\prime}$ has at most 5 elements by Lemma 4.3.5, so $\left|E\left(M^{\prime}\right)\right| \leq 9$. Hence $|E(M)| \leq 11$.

Suppose that $M^{\prime}$ or $\left(M^{\prime}\right)^{*}$ is obtained from $U_{2,5}$ by gluing wheels to triangles. Then there are at most three corresponding fans $F_{1}, F_{2}$ and $F_{3}$ of $M^{\prime}$, and each fan $F_{i}$ has at most five elements for $i \in\{1,2,3\}$, so $\left|E\left(M^{\prime}\right)\right| \leq 15$. Thus $|E(M)| \leq 17$. The result now follows from Theorem 4.2.4.

\subsection{Blocking a triad}

It remains to bound the fragile matroids with an $\left\{X_{8}, Y_{8}, Y_{8}^{*}\right\}$-minor. The goal in this section is to establish that $M^{\prime}$ has a path sequence with a triad end $P_{1}$, and that $a$ or $b$ must block the 3 -separation $\left(P_{1}, \ldots, P_{n}\right)$.

The next lemma follows easily from the fact that the matroids in $\left\{X_{8}, Y_{8}, Y_{8}^{*}\right\}$ have no essential elements.

Lemma 4.4.1. If $M^{\prime}$ has an $\left\{X_{8}, Y_{8}, Y_{8}^{*}\right\}$-minor, then $M^{\prime}$ has no essential elements.

We build towards a proof that $M^{\prime}$ has a triad end.

Lemma 4.4.2. If $M^{\prime}$ has a triad $T$, then either $T$ is allowable or $T$ meets $\{x, y\}$. 
Proof. Suppose that $M^{\prime}$ has a triad $T$ such that all the elements of $T$ are nondeletable. Then every element of $T$ is contractible because $M^{\prime}$ has no essential elements. Then $T$ cannot be a subset of the cobasis $B^{*}-\{a, b\}$, so $B$ must contain an element of $T$. Since all the elements of $T$ are contractible, and the elements of $B-\{x, y\}$ are not contractible, it follows that $T \cap B \subseteq\{x, y\}$.

Now, the end step of the path sequence for $M^{\prime}$ could be a triad, a 4-element cosegment, or a fan with at most one triangle. We consider the 4-element cosegment case next.

Lemma 4.4.3. If $|E(M)| \geq|E(N)|+17$, and $M^{\prime}$ has an $(N, B)$-robust element outside of $\{x, y\}$, then $M^{\prime}$ has no 4-element cosegment. Moreover, if $|E(M)| \geq$ $|E(N)|+17$, and $M^{\prime}$ has a path sequence with at least three steps, then $M$ has a path sequence with an end that is not a 4-cosegment.

Proof. Suppose that $|E(M)| \geq|E(N)|+17$ and that $M^{\prime}$ has an $(N, B)$-robust element $z$ outside of $\{x, y\}$. Let $C$ be a 4-element cosegment of $M^{\prime}$. Then $C$ contains triad $T=\{q, r, s\}$ that meets $\{x, y\}$ by Lemma 4.4.2. But now $T \cup\{x, y, z\}$ is a gadget of $M^{\prime}$, so $|E(M)| \leq|E(N)|+16$ by Lemma 4.2.12, a contradiction.

Now suppose that $|E(M)| \geq|E(N)|+17$ and that $M^{\prime}$ has a path sequence with at least three steps such that each end is a 4-cosegment. We may assume that $M^{\prime}$ has no $(N, B)$-robust elements outside of $\{x, y\}$. Let $C$ and $C^{\prime}$ be the 4-cosegment ends of $M^{\prime}$. Now, if $\{x, y\} \subseteq C$, then either $M^{\prime}$ has a gadget or $C \cup C^{\prime}$ is a corank-3 set such that $\left(C \cup C^{\prime}\right) \cap\left(B^{*}-\{a, b\}\right)$ cospans $x$ and $y$. But then $M^{\prime}$ has an internal coguts element $c \in B^{*}-\left(C \cup C^{\prime}\right)$ because $M^{\prime}$ has a path sequence with at least three steps, and $A_{x c}=A_{y c}=0$ because $\left(C \cup C^{\prime}\right) \cap\left(B^{*}-\{a, b\}\right)$ cospans $x$ and $y$. Then a pivot on $A_{p c}$ is allowable for any $A_{p c} \neq 0$, and the basis $B \triangle\{c, p\}$ contradicts the fact that $B$ is a robust basis. We may therefore assume that $|C \cap\{x, y\}|=1$ by Lemma 4.4.2. Assume that $x \in C$. Moreover, the deletable element $p$ of $C$ is in $B-\{x, y\}$ and $C-\{p, x\}$ is contained in $B^{*}-\{a, b\}$ because $M^{\prime}$ has no $(N, B)$ robust elements. By the same argument, $\left|C^{\prime} \cap\{x, y\}\right|=1$. Suppose that $c \in C^{\prime}$. Again, the deletable element $p^{\prime}$ of $C^{\prime}$ is in $B-\{x, y\}$ and $C-\left\{p^{\prime}, x\right\}$ is contained in $B^{*}-\{a, b\}$ because $M^{\prime}$ has no $(N, B)$-robust elements. But then $C \cup C^{\prime}$ is a corank3 set with four elements in $B^{*}-\{a, b\}$; a contradiction. Thus we can assume that $y \in C^{\prime}$. But $M^{\prime}$ has a path sequence with at least three steps, so there is some coguts element $w$ of $M^{\prime}$ outside of $C \cup C^{\prime}$. Then $w \in B^{*}-C \cup C^{\prime}$ because $M^{\prime}$ has 
no $(N, B)$-robust elements. Now $A_{x w}=A_{y w}=0$ because $x, y \in \mathrm{cl}^{*}\left(B^{*} \cap\left(C \cup C^{\prime}\right)\right)$, and $A_{s w} \neq 0$ for some $s \in B-\{x, y\}$ because $w$ is not a loop of $M^{\prime}$. But then a pivot on $A_{s w}$ is allowable, and the basis $B \triangle\{s, w\}$ has a robust element outside of $\{x, y\}$; a contradiction because $B$ is a robust basis.

We can now show that if a robust excluded minor is sufficiently large, then $M^{\prime}$ must have a path sequence ending in a triad. Moreover, we can assume that $B$ is chosen so that the triad contains no $(N, B)$-robust element of $M^{\prime}$.

Lemma 4.4.4. If $|E(M)| \geq|E(N)|+17$, and $M^{\prime}$ has a path sequence with at least three steps, then $M^{\prime}$ has a path sequence with a triad end $T$. We can assume that $B$ is chosen such that $T$ has no $(N, B)$-robust elements of $M^{\prime}$.

Proof. We first show that $M^{\prime}$ has a path sequence with a triad end. Suppose $M^{\prime}$ has a path sequence ending in a 4-cosegment. Then it follows from Lemma 4.4.3 and Lemma 4.3 .4 that other end is not a 4-element cosegment, and it cannot contain a triangle. Thus $M^{\prime}$ has a triad end. Now suppose that $M^{\prime}$ has no 4-cosegment end. Then at most one end can be a fan with $|F| \geq 4$, so again the other end is a triad. By rearrangement, we can assume that $M^{\prime}$ has a path sequence ending in a triad.

Suppose that $x \in P_{1}$. If $M^{\prime}$ has an $(N, B)$-robust element outside of $\{x, y\}$, then $\{x, y, z\}$ is a triad of $M^{\prime}$, and $\{x, y, z\} \subseteq P_{1} \cup P_{2} \cup P_{3}$ by orthogonality. But $M^{\prime}$ has a path sequence with at least three steps, so the other end step does not meet $\{x, y\}$, and it cannot be a 4-cosegment or a fan. Thus by rearrangement $M^{\prime}$ has a path sequence ending in a triad with no $(N, B)$-robust elements of $M^{\prime}$. Suppose that $M^{\prime}$ has no $(N, B)$-robust elements outside of $\{x, y\}$. Then $y \notin P_{1}$ by Lemma 4.3.1 and the fact that $M^{\prime}$ has no $(N, B)$-robust element outside of $\{x, y\}$. Now $P_{1}$ contains at most one element $q$ of $B^{*}-\{a, b\}$, so there is some $w \in B^{*}-\{a, b, q\}$ such that $A_{x w} \neq 0$. Then $B \triangle\{w, x\}$ is a robust basis for $M^{\prime}$ with the desired property.

By Lemma 4.4.4, we assume that $M^{\prime}$ has a triad end $P_{1}$ such that $P_{1}$ contains no $(N, B)$-robust elements of $M^{\prime}$. We assume that $M^{\prime}$ does not satisfy the bounds of Theorem 4.2.11.

Recall that an element $x$ blocks a 3-separation $(A, B)$ of $M \backslash x$ if $x \notin \operatorname{cl}_{M}(A)$ and $x \notin \mathrm{cl}_{M}(B)$. 
Lemma 4.4.5. If $M^{\prime}$ has a path sequence ending in a triad $P_{1}$ with no $(N, B)$-robust elements of $M$, then at least one of $a$ and b blocks the 3-separation $\left(P_{1}, P_{2} \cup \cdots \cup\right.$ $\left.P_{n}\right)$ of $M^{\prime}$.

Proof. Assume that $M^{\prime}$ has a path sequence ending in a triad $P_{1}$ with no $(N, B)$ robust elements of $M$. Then $P_{1}=\{p, q, r\}$ for some contractible elements $p, q \in$ $B^{*}-\{x, y\}$ and a deletable element $r \in B-\{x, y\}$.

We first show that neither $a$ nor $b$ is in $\operatorname{cl}_{M}\left(P_{1}\right)$. Seeking a contradiction, suppose that $a \in \mathrm{cl}_{M}\left(P_{1}\right)$.

4.4.5.1. Up to allowable pivots, there is a basis $B^{\prime}$ for $M$ with incriminating set $\left\{a, b, x^{\prime}, y^{\prime}\right\}$ for $\left(M, A^{\prime}\right)$ such that $x^{\prime} \in P_{1}$ and $y^{\prime} \in P_{3} \cup \cdots P_{n}$.

Subproof. Suppose that $A_{x p}=A_{y p}=0$. Then a pivot on $A_{r p} \neq 0$ is allowable, and the basis $B \triangle\{p, r\}$ has more robust elements outside of $\{x, y\}$ than $B$; a contradiction because $B$ is a robust basis. Thus we can assume that $A_{x p} \neq 0$. Then a pivot on $A_{x p}$ is allowable, and gives the required basis.

Now $P_{1} \cup P_{2}$ is a rank-3 circuit of $M^{\prime}$ that contains three basis elements $p, q, x^{\prime} \in B^{\prime}$. Thus $a \in \operatorname{cl}_{M}\left(\left\{p, q, x^{\prime}\right\}\right)$, so it follows that $A_{t a} \neq 0$ if and only if $t \in P_{1} \cup P_{2}$. In particular, it follows that $A_{y^{\prime} a}=0$; a contradiction because there are no zero-entries in the bad submatrix. Therefore $a \notin \mathrm{cl}_{M}\left(P_{1}\right)$, and by a symmetric argument we also conclude that $b \notin \mathrm{cl}_{M}\left(P_{1}\right)$.

Next we show that $a \notin \mathrm{cl}_{M \backslash b}\left(P_{2} \cup \cdots \cup P_{n}\right)$ or $b \notin \mathrm{cl}_{M \backslash a}\left(P_{2} \cup \cdots \cup P_{n}\right)$. Suppose that $a \in \mathrm{cl}_{M \backslash b}\left(P_{2} \cup \cdots \cup P_{n}\right)$ and $b \in \mathrm{cl}_{M \backslash a}\left(P_{2} \cup \cdots \cup P_{n}\right)$. Then $a \notin \operatorname{cl}_{M^{*}}\left(P_{1} \cup b\right)$ and $b \notin \mathrm{cl}_{M^{*}}\left(P_{1} \cup a\right)$, so the triad $P_{1}$ of $M \backslash a, b$ is also a triad of $M$. Thus $\nabla_{P_{1}}(M)$ is a robust excluded minor, and $\nabla_{P_{1}}(M) \backslash a, b=\nabla_{P_{1}}(M \backslash a, b)$, so $a, b$ is a pair of elements for $\nabla_{P_{1}}(M)$ such that $\nabla_{P_{1}}(M) \backslash a, b$ is 3 -connected with a $U_{2,5}$-minor. But $\nabla_{P_{1}}(M) \backslash a, b$ has a 4-element segment $P_{1} \cup P_{2}$. Since at most two elements of $P_{1} \cup P_{2}$ can be in a robust basis $B^{\prime}$ for $\nabla_{P_{1}}(M) \backslash a, b$, it follows that $\nabla_{P_{1}}(M) \backslash a, b$ has at least one $\left(N, B^{\prime}\right)$-robust element $z \in\left(B^{\prime}\right)^{*}-\{a, b\}$ outside of $\left\{x^{\prime}, y^{\prime}\right\}$ with $z \in P_{1} \cup P_{2}$. But then $\left\{x^{\prime}, y^{\prime}, z\right\}$ is a triad of $\nabla_{P_{1}}(M) \backslash a, b$ by Theorem 4.2.10; a contradiction of orthogonality.

We may therefore assume that $a$ blocks the 3-separation $\left(P_{1}, P_{2} \cup \cdots \cup P_{n}\right)$ of $M^{\prime}$. 


\subsection{New pairs for deletion and contraction}

In this section, we assume that $a$ blocks the triad $P_{1}$ of $M^{\prime}$, and we look for new pairs of elements to either delete or contract that preserve the property that $a$ blocks the triad $P_{1}$. To find these pairs, we need to understand the structure of circuits and cocircuits relative to the path of 3 -separations $\left(P_{1}, \ldots, P_{n}\right)$.

It is relatively easy to show that an internal delete pair bounds $M$. We say that $M$ is in a bounded equivalence class if there is some excluded minor $M^{\prime}$ that is $\Delta$ - $\nabla$-equivalent to $M$ or $M^{*}$ such that $\min \left\{r\left(M^{\prime}\right), r^{*}\left(M^{\prime}\right)\right\} \leq 10$.

Lemma 4.5.1. Let $P_{i}$ and $P_{j}$ be internal guts steps of the path $\left(P_{1}, \ldots, P_{n}\right)$, and let $s \in P_{i}$ and $t \in P_{j}$. If $M$ is not in a bounded equivalance class, then $M^{\prime} \backslash s, t$ is not 3-connected.

Proof. Suppose that $M^{\prime} \backslash s, t$ is 3-connected. Then $M \backslash s, t$ is 3-connected. Suppose that $M \backslash s, t$ is fragile. Then the fragile matroid $M^{\prime} \backslash s, t$ has an extension $M \backslash s, t$ such that $a$ blocks the end triad $P_{1}$ of $M \backslash s, t$; contradiction of Theorem 4.2.5. Thus, by Theorem 4.2.10, the matroid $M \backslash s, t$ is not fragile, and $C^{\prime}=\left\{s, t, x^{\prime}, y^{\prime}, z^{\prime}\right\}$ is a 5-element cocircuit that contains a triangle $T^{\prime}=\left\{s, x^{\prime}, y^{\prime}\right\}$. Now, neither $a$ nor $b$ is a member of $C^{\prime}$ because $\left\{x^{\prime}, y^{\prime}, z^{\prime}\right\}$ is a triad of $M \backslash s, t$, but $M^{\prime} \backslash s, t$ is 3-connected. Thus $C^{\prime}$ is a 5-element cocircuit of $M^{\prime}$ that contains a triangle $T^{\prime}$ of $M^{\prime}$. By Lemma 4.3.4 $M^{\prime}$ has an $(N, B)$-robust element $z$, and $T^{\prime}$ contains $z$ and one of $\{x, y\}$. Since $\{x, y, z\}$ is a triad and $C^{\prime}$ a 5-element cocircuit of $M^{\prime}$, the elements $x$ and $y$ are not both in $C^{\prime}$. But $M^{\prime}$ is fragile, and therefore 3-connected up to series classes, so the element $z$ is an $(N, B)$-strong of $M^{\prime}$, and $\{b, x, y\}$ is a triangle of $M$; a contradiction of orthogonality because $\{b, x, y\}$ meets the cocircuit $C^{\prime}$ in one element.

Since $M^{\prime}$ is 3-connected up to series and parallel classes, Lemma 4.5.1 shows that $M^{\prime}$ must have lots of small cocircuits meeting the internal steps of the path $\left(P_{1}, \ldots, P_{n}\right)$. Intuitively, we should therefore be able to find pairs of elements to contract.

We call a circuit or cocircuit $C$ of $M^{\prime}$ path conforming if $C \subseteq P_{1} \cup \cdots \cup P_{i}$ for some $i<n$. Let $C$ be a path-conforming circuit or cocircuit. If $C \cap P_{i} \neq \emptyset$ and $C \cap P_{i+1}=\emptyset$, then we call the members of $C \cap P_{i}$ the ends of $C$.

Next lemma is an easy consequence of orthogonality. 
Lemma 4.5.2. Let $C$ be a path-conforming circuit of $M^{\prime}$. Then the ends of $C$ are guts elements of the path $\left(P_{1}, \ldots, P_{n}\right)$.

Proof. Suppose that the elements of $C \cap P_{i}$ are coguts elements. Let $p \in C \cap P_{i}$. Then $p$ is in the coclosure of $P_{1} \cup \cdots \cup P_{i-1} \cup\left(P_{i}-\{p\}\right)$ by definition of the path, and $p \in C \subseteq P_{1} \cup \cdots \cup P_{i}$, so $p$ is also in the closure of $P_{1} \cup \cdots \cup P_{i-1} \cup\left(P_{i}-\right.$ $\{p\})$. But both $P_{1} \cup \cdots \cup P_{i-1} \cup\left(P_{i}-\{p\}\right)$ and $P_{1} \cup \cdots \cup P_{i}$ are 3-separating sets; a contradiction of Lemma 4.2.2.

The following result is an easy consequence of the property that a fragile matroid is 3-connected up to series and parallel classes.

Lemma 4.5.3. Let $v, w$ be internal coguts elements of the path $\left(P_{1}, \ldots, P_{n}\right)$ such that $M^{\prime} / v, w$ is 3-connected. If $M$ has no triangle that meets both $\{a, b\}$ and $\{v, w\}$, and no 4-element circuit that contains $\{v, w\}$ and meets $\{a, b\}$, then $M / v, w$ is 3 connected.

Proof. Suppose that $M$ has no triangle that meets both $\{a, b\}$ and $\{v, w\}$, and no 4-circuit that contains $\{v, w\}$ and meets $\{a, b\}$

Now consider $M \backslash a / v$. Since $M \backslash a$ and $M \backslash a, b / v$ are 3-connected, it follows that $M \backslash a / v$ is 3-connected up to a parallel pair that contains $b$. Suppose that $b$ is in a parallel pair of $M \backslash a / v$. Then $M \backslash a$ has a triangle $T$ such that $b, v \in T$. But then $T$ is a triangle of $M$ that meets both $\{a, b\}$ and $\{v, w\}$; a contradiction. Thus $M \backslash a / v$ is 3-connected. Consider $M / v$. Then it follows from the fact that $M$ and $M \backslash a / v$ are 3-connected that $M / v$ is 3-connected up to a parallel pair that contains $a$. But if $M / v$ has a parallel pair that contains $a$, then $M$ has a triangle that contains both $v$ and $a$; a contradiction. Thus $M / v$ is 3-connected. By the same argument $M / w$ and $M \backslash a / w$ are 3-connected.

Now consider $M \backslash a / v, w$. Since $M \backslash a / v$ and $M \backslash a, b / v, w$ is 3-connected, it follows that $M \backslash a / v, w$ is 3-connected up to a parallel pair that contains $b$. Suppose $b$ is in a parallel pair of $M \backslash a / v, w$. Then $M \backslash a / v$ has a triangle $T$ such that $b, w \in T$. Thus either $T$ is a triangle that meets both $\{a, b\}$ and $\{v, w\}$ or $T \cup\{v\}$ is a circuit of $M$ that contains $\{v, w\}$ and meets $\{a, b\}$; a contradiction. Thus $M \backslash a / v, w$ is 3connected. Finally, consider $M / v, w$. Now $M / v$ and $M \backslash a / v, w$ are 3-connected, so either $M / v, w$ is 3-connected or it has a parallel pair that contains $a$. Suppose that $a$ is in a parallel pair of $M / v, w$. Then $M / v$ has a triangle $T$ such $a, w \in T$. Hence 
$T$ is triangle of $M$ that meets both $\{a, b\}$ and $\{v, w\}$ or $T \cup\{v\}$ is a 4-element circuit of $M$ that contains $\{v, w\}$ and meets $\{a, b\}$; a contradiction. Thus $M / v, w$ is 3-connected.

A large coguts step $P_{i}$ makes the adjacent guts lines $P_{i-1}$ and $P_{i+1}$ skew, and thus restricts the path-conforming circuits running through $P_{i}$.

Lemma 4.5.4. Let $P_{j}$ be an internal coguts step of the path $\left(P_{1}, \ldots, P_{n}\right)$ of $M^{\prime}$, and let $w_{1}, w_{2}, w_{3}$ be internal coguts elements of $M^{\prime}$ such that $M^{\prime} / w_{i}$ is 3-connected, $w_{1}, w_{2} \in P_{1} \cup \cdots \cup P_{j-1}$ and $w_{3} \in P_{j+1} \cup \cdots \cup P_{n}$. If $\left|P_{j}\right| \geq 2$, then $M^{\prime}$ has no 4element circuit $C$ that contains $w_{3}$ and meets $\left\{w_{1}, w_{2}\right\}$. Moreover, if $\left|P_{j}\right| \geq 2$ and $M^{\prime} / w_{1}, w_{2}$ is 3-connected, then there is no 5-element circuit $C$ of $M^{\prime}$ that contains $\left\{w_{1}, w_{2}, w_{3}\right\}$.

Proof. Suppose that $\left|P_{j}\right| \geq 2$. Assume that $M$ has a 4-element circuit $C=$ $\left\{w_{1}, w_{3}, p, q\right\}$, and that $w_{1} \in P_{i}, w_{3} \in P_{k}$ where $i<j<k$. Then $p \in P_{1} \cup \cdots \cup P_{i-1}$ and $q \in P_{k+1}$. Now $M^{\prime} / w_{1}$ has a triangle $\left\{p, q, w_{3}\right\}$. So the guts line $P_{i+1}$ meets the guts line $P_{j+1}$ in $M^{\prime} / w_{1}$. But these guts lines are skew in $M^{\prime}$, and hence in $M^{\prime} / w_{1}$ because $\left|P_{j}\right| \geq 2$; a contradiction. The same argument shows there is no 5-element circuit $C$ of $M^{\prime}$ that contains $\left\{w_{1}, w_{2}, w_{3}\right\}$ when $M^{\prime} / w_{1}, w_{2}$ is 3 -connected.

We make the following observation on path-conforming triads.

Lemma 4.5.5. Let $M \in \mathscr{P}$, and $w$ be an internal coguts element. If $T$ is a triad of $M$ such that $w \in T$, then either $T$ is a step or T meets an adjacent guts step and the next coguts step.

Now for finding contract pairs, we have the following first step.

Lemma 4.5.6. Let $3 \leq i \leq j$, and $n \geq j+10$. Let $P_{i}$ be an internal coguts steps of the path $\left(P_{1}, \ldots, P_{n}\right)$, and let $v \in P_{i}$. There is some $w \in P_{k}$ for $k \in\{j+2, j+4, j+$ $6, j+8\}$ such that $M^{\prime} / v, w$ is 3-connected.

Proof. We can assume that $\left|P_{k}\right|=1$ for $k \in\{j+2, j+4, j+6\}$ or it follows from Lemma 4.5.4 that there is such a pair. Let $w_{i} \in P_{j+2 i}$ for $i=1,2,3$. Now, since $M^{\prime} / v, w_{i}$ is not 3-connected, there are 4-element circuits $C_{v, w_{i}}$ of $M^{\prime}$ such that $v, w_{i} \in C_{v, w_{i}}$ for $i \in\{1,2,3\}$. Now, the path-conforming circuit $C_{v, w_{i}}$ meets a guts element of $P_{1} \cup \cdots \cup P_{i-1}$ and a guts element of $P_{i+2 i+1}$. But now let $s \in P_{j+3} \cap C_{v, w_{1}}$ 
and $t \in P_{j+5} \cap C_{v, w_{2}}$. Then $M^{\prime} \backslash s, t$ is not 3-connected by Lemma 4.5.1. Thus $s$ or $t$ is in a triad of $M^{\prime}$, or $s, t \in C^{\prime}$ for some 4-element cocircuit of $M^{\prime}$. If $s$ is in a triad $T^{\prime}$, then $w_{1}, w_{2} \in T^{\prime}$, so $T^{\prime}$ meets $C_{v, w_{2}}$ in a single element; a contradiction of orthogonality. Similarly, if $t$ is in a triad $T^{\prime}$, then $w_{2}, w_{3} \in T^{\prime}$, so $T^{\prime}$ meets $C_{v, w_{3}}$ in a single element; a contradiction of orthogonality. Thus $s, t \in C^{\prime}$ for some 4element cocircuit of $M^{\prime}$. Now since $C^{\prime}$ is path-conforming $w_{3} \in C^{\prime}$ and $C^{\prime}$ meets some contractible element of $P_{1} \cup \cdots \cup P_{i-1}$. Thus $C^{\prime}$ meets $C_{v, w_{i}}$ for $i \in\{1,2,3\}$, so $C^{\prime}=\left\{v, s, t, w_{3}\right\}$ by orthogonality. But now $M^{\prime}$ has no 4-element circuit containing $v$ and $w_{4}$ for $w_{4} \in P_{j+8}$. Hence $M^{\prime} / v, w_{4}$ is 3-connected.

By Lemma 4.5.3, we need to bound the number of pairs that are contained in 3or 4-element circuits of $M$ with $a$ or $b$.

Let $m \geq 3$. Suppose $P_{i}$ is an internal coguts element of the path $\left(P_{1}, \ldots, P_{n}\right)$ of $M^{\prime}$, where $n \geq i+2(m+2)+2$. We call the subset $P_{i+2} \cup P_{i+3} \cup \cdots \cup P_{i+2(m+2)}$ of $E\left(M^{\prime}\right)-\{x, y\}$ an $m$-block for $P_{i}$. Roughly speaking, an $m$-block can be thought of as the search space for an internal contract pair.

Lemma 4.5.7. Let $m \geq 2$, and let $w_{1}, \ldots, w_{m}$ be internal coguts elements of $M^{\prime}$ such that $M^{\prime}$ has no 4-element circuit that contains $\left\{w_{i}, w_{j}\right\}$, and no 5-element circuit that contains $\left\{w_{1}, w_{i}, w_{j}\right\}$ for all $i, j \in\{1, \ldots, m\}$. If $w_{m} \in P_{h}$ and $M^{\prime}$ has an $m$-block for $P_{i}$ for some $i>h$, then there is an internal coguts element $w_{m+1}$ in the m-block for $P_{i}$ such that $M^{\prime}$ has no 4-element circuit that contains $\left\{w_{i}, w_{m+1}\right\}$ for all $i \in\{1, \ldots, m\}$ and no 5-element circuit that contains $\left\{w_{1}, w_{j}, w_{m+1}\right\}$ for all $j \in\{2, \ldots, m\}$.

Proof. Suppose that $\left|P_{i+2 k}\right| \geq 2$ for some $k<m+1$. Then it follows from Lemma 4.5.4 that $w_{m+1} \in P_{i+2(m+1)}$ has the desired property. We may therefore assume that $\left|P_{i+2 k}\right|=1$ for all $k<m+1$. Suppose there is no $w \in P_{i+2 k}$ with the required property for each $k<m+1$. Then for each $k$ there is a 4- or 5-element circuit $C_{k}$ that meets $P_{i+2 k}, P_{i+2 k+1}$, either a single $w_{i}$ or pair $w_{1}, w_{i}$ and some guts element of $P_{1} \cup \cdots \cup P_{i-1}$.

Now consider pairs of guts elements $s \in P_{i+2 k+1} \cap C_{k}$ and $t \in P_{i+2 k+3} \cap C_{k+1}$ for each $k<m+1$. By orthogonality with $C_{k+1}$, the element $s$ is not in a triad of $M^{\prime}$. Similarly, $t$ is not in a triad by orthogonality with $C_{k+2}$. Thus it follows from Lemma 4.5.1 and orthogonality that $M^{\prime}$ has a 4-element cocircuit $C_{k}^{*}$ that contains $s, t$ and meets $P_{i+2 k+4}$ and some coguts element of $P_{1} \cup \cdots \cup P_{i-1}$. But $C_{k}^{*}$ meets 
$C_{k+1}, C_{k+2}, C_{k+3}$ and some coguts element of $P_{1} \cup \cdots \cup P_{i-1}$. By orthogonality with $C_{k+1}, C_{k+2}, C_{k+3}$, the coguts element of $C^{*}$ in $P_{1} \cup \cdots \cup P_{i-1}$ must some $w_{i}$ that is in each of $C_{k+1}, C_{k+2}, C_{k+3}$ but no other $C_{\ell}$ for $\ell \notin\{k+1, k+2, k+3\}$. Thus each $w_{i}$ is in exactly one trio of circuits $C_{k+1}, C_{k+2}, C_{k+3}$.

Now let $w_{m+1} \in P_{i+2(m+2)}$. Now if $M^{\prime}$ has a 4-element circuit $C$ that contains $\left\{w_{i}, w_{m+1}\right\}$ or a 5 -element circuit $C^{\prime}$ that contains $\left\{w_{1}, w_{j}, w_{m+1}\right\}$ for all $j \in\{1, \ldots, m\}$, then $C$ and $C^{\prime}$ meet some cocircuit $C_{k}^{*}$ in a single element; a contradiction of orthogonality. Thus there is some $w_{m+1}$ with the desired properties.

We can now bound the triangles that meet $\{a, b\}$ and internal coguts steps. In particular, we use this result to find the first element of our contract pairs.

Lemma 4.5.8. $M^{\prime}$ has at most five internal coguts elements that are contained in a triangle of $M$ that also meets $\{a, b\}$. Moreover, if $n \geq 21$, then $M^{\prime}$ has an internal guts element in a step $P_{i}$ with $i \leq 19$ such that $M^{\prime} / w_{1}$ is 3-connected, $w_{1} \notin \mathrm{cl}_{M}\left(P_{1} \cup\{a\}\right)$, and $M$ has no triangle that contains $w_{1}$ and meets $\{a, b\}$.

Proof. We first claim that there is some internal coguts element, $w_{1}$, such that $w_{1}$ is not in $\{x, y\}$, so that $M^{\prime} / w_{1}$ is 3-connected. This follows from the fact that $\{x, y\}$ are not deletable and if $M^{\prime}$ has a triangle, then $M^{\prime}$ meets $\{x, y\}$. Thus if an internal coguts element $w$ is in a triangle $T$, then $w$ is the only contractible element of the triangle, so $w \in\{x, y\}$.

There is at most one internal coguts element $w$ of $M^{\prime}$ contained in a triangle of $M$ of the form $\{a, b, w\}$. Suppose that $v, w$ are distinct internal coguts elements of $M^{\prime}$ such that $\{a, b, v\}$ and $\{a, b, w\}$ are triangles of $M$. Then $\{a, b, v, w\}$ is a 4-segment of $M$, so $\{a, v, w\}$ is a triangle of $M$. But then $a \in \operatorname{cl}_{M \backslash b}\left(P_{2} \cup \cdots \cup P_{n}\right)$; a contradiction because $a$ blocks the 3-separation $\left(P_{1}, P_{2} \cup \cdots \cup P_{n}\right)$ of $M^{\prime}$.

We also claim there is at most one triangle of the form $\{a, p, w\}$ for $p, w \in E\left(M^{\prime}\right)$, where $w$ is an internal coguts element if $M^{\prime}$. Suppose there are two triangles $\{a, p, w\}$ and $\left\{a, p^{\prime}, w^{\prime}\right\}$, where $w$ and $w^{\prime}$ are internal coguts elements of $M^{\prime}$. Then $p, p^{\prime} \in P_{1}$ because $a$ blocks the 3 -separation $\left(P_{1}, P_{2} \cup \cdots \cup P_{n}\right)$ of $M^{\prime}$. But then $P_{1} \cup a$ spans both $w$ and $w^{\prime}$; a contradiction because $r\left(P_{1} \cup a\right)=r\left(P_{1}\right)+1$ but $r\left(P_{1} \cup\left\{w, w^{\prime}\right\}\right)=r\left(P_{1}\right)+2$.

Finally, consider triangles of the form $\{b, p, w\}$ for $p, w \in E\left(M^{\prime}\right)$, where $w$ is an internal coguts element of $M^{\prime}$. We may assume that $w_{1}$ is not in $\{x, y\}$, so that 
$w_{1} \in B^{*}-\{a, b\}$ since $w_{1}$ is contractible. Suppose that $p \in B-\{x, y\}$. Then we can perform an allowable pivot on $A_{x w}$ or $A_{y w}$, and then $b$ is spanned by $p$ and $w$, so that $A_{y b}^{x w}=0$; a contradiction because $A^{x w}$ cannot have zero-entries in the bad submatrix $A^{x w}[\{a, b, w, y\}]$. Thus either $p \in\{x, y\}$ or $p \in B^{*}-\{a, b\}$. In either case $p$ is contractible in $M^{\prime}$. Now suppose that $M$ has two such triangles $\{b, p, w\}$ and $\left\{b, p^{\prime}, w^{\prime}\right\}$. Then $M^{\prime}$ has a circuit $C$ contained in $\left\{p, p^{\prime}, w, w^{\prime}\right\}$, so every element of $C$ is contractible in $M^{\prime}$; a contradiction because $M^{\prime}$ is fragile. Thus $M$ has at most one triangle of the form $\{b, p, w\}$ for $p, w \in E\left(M^{\prime}\right)$, where $w$ is an internal coguts element of $M^{\prime}$.

Now, suppose $n \geq 21$. Then it follows that $M^{\prime}$ has at least nine internal coguts steps. At most two of these steps are in $\{x, y\}$, at most one step is spanned by $P_{1} \cup\{a\}$, at most one step is in an $\{a, b\}$ triangle, at most two steps are in a triangle of the form $\{a, p, w\}$, and at most two steps are in a triangle of the form $\{b, p, w\}$. That is, at most eight internal coguts steps fail. Thus $M^{\prime}$ must have a required $w_{1}$ and $w_{1} \in P_{i}$ for some $i \leq 19$.

Next we bound the number of our potential contract pairs that could be contained in 4-element circuits that meet $\{a, b\}$.

Lemma 4.5.9. Let $w_{1}, \ldots, w_{m}$ be internal coguts elements of the path $\left(P_{1}, \ldots, P_{n}\right)$ of $M^{\prime}$ such that $M^{\prime} / w_{1}, w_{i}$ is 3-connected for all $i \in\{1, \ldots, m\}$, and $M^{\prime}$ has no 4-element circuit that contains $\left\{w_{i}, w_{j}\right\}$ and no 5-element circuit that contains $\left\{w_{1}, w_{i}, w_{j}\right\}$ for all $i, j \in\{1, \ldots, m\}$. Then $M$ has at most three 4-element circuits that contain $\left\{w_{1}, w_{i}\right\}$ and meet $\{a, b\}$.

Proof. We first consider 4-element circuits of $M$ of the form $\left\{a, b, w_{1}, w_{i}\right\}$. Suppose there are at least two such circuits, $\left\{a, b, w_{1}, w_{i}\right\}$ and $\left\{a, b, w_{1}, w_{j}\right\}$. Then by elimination on $b, M$ has a circuit $C$ contained in $\left\{a, w_{1}, w_{i}, w_{j}\right\}$. Now if $a \notin C$, then $C$ is a circuit of $M^{\prime}$; a contradiction because $M^{\prime} / w_{1}, w_{i}$ is 3-connected. Thus $a \in C$. But then $a \in \mathrm{cl}_{M}\left(P_{2} \cup \cdots \cup P_{n}\right)$; a contradiction because $a$ blocks $\left(P_{1}, P_{2} \cup \cdots \cup P_{n}\right)$. Thus $M$ has at most one 4-element circuit of the form $\left\{a, b, w_{1}, w_{i}\right\}$.

Now we consider 4-element circuits of $M$ of the form $\left\{a, w_{1}, w_{i}, p\right\}$ for some $p \neq$ $b$. Suppose there are at least two such circuits, $\left\{a, w_{1}, w_{i}, p\right\}$ and $\left\{a, w_{1}, w_{j}, q\right\}$. Now $p, q \in P_{1}$ or $a \in \operatorname{cl}_{M}\left(P_{2} \cup \cdots \cup P_{n}\right)$. Now by elimination on $a$ it follows that $M$ has a circuit $C$ in $\left\{w_{1}, w_{i}, w_{j}, p, q\right\}$. Now at least one of $p, q$ are in $C$ because 
$M^{\prime} / w_{1}, w_{i}$ is 3 -connected. But now $C$ is a path-conforming circuit that ends in an internal coguts element; a contradiction.

Finally, we consider 4-element circuits of $M$ of the form $\left\{b, w_{1}, w_{i}, p\right\}$ for some $p \neq a$. Assume that there are at least two such circuits, $\left\{b, w_{1}, w_{i}, p\right\}$ and $\left\{b, w_{1}, w_{j}, q\right\}$. Then by elimination on $b$ it follows that $M$ has a circuit contained in $\left\{w_{1}, w_{i}, w_{j}, p, q\right\}$; a contradiction because $M^{\prime}$ has no 4-element circuit that contains $\left\{w_{i}, w_{j}\right\}$ and no 5-element circuit that contains $\left\{w_{1}, w_{i}, w_{j}\right\}$ for all $i, j$.

We can now find our pairs of elements to contract. The strategy is to repeatedly apply Lemma 4.5.7 to find potential pairs to contract. It follows from Lemma 4.5.9 and Lemma 4.5.9 that at most a small number of these pairs can be in small circuits that meet $\{a, b\}$, so we simply find more pairs than the possible number of pairs that can be in such circuits. To be more precise, we find at least three pairs that avoid small circuits and preserve the property that $a$ blocks $P_{1}$, the reason for which will become clear in the following section.

Lemma 4.5.10. Let $\left(P_{1}, \ldots, P_{n}\right)$ be a path for $M^{\prime}$, where $n \geq 663$. There are internal coguts elements $v, w_{1}, w_{2}, w_{3} \in E\left(M^{\prime}\right)-\{x, y\}$ such that $M^{\prime} / v, w_{i}$ and $M / v, w_{i}$ are 3-connected with an $N$-minor for $i \in\{1,2,3\}$, and a blocks the triad $P_{1}$ of $M^{\prime} / v, w_{i}$.

Proof. We claim there are internal coguts elements $w_{1}, w_{2}, \ldots, w_{13} \in E\left(M^{\prime}\right)-$ $\{a, b, x, y\}$ such that:

(i) $w_{1} \in P_{k_{1}}$ for some $k_{1} \leq 19, M^{\prime} / w_{1}$ is 3-connected, $w_{1} \notin \mathrm{cl}_{M}\left(P_{1} \cup\{a\}\right)$, and $M$ has no triangle that contains $w_{1}$ and meets $\{a, b\}$; and

(ii) For all $i \in\{2, \ldots, 12\}$ there is some $P_{k_{i}}$ such that $w_{i} \in P_{k_{i}}$, where $k_{2} \leq 47$ and $k_{j} \leq k_{j-1}+3(2 j+2)+2$ for $j \in\{3, \ldots, 13\}$; and

(iii) $M^{\prime} / w_{1}, w_{i}$ is 3 -connected for all $i \in\{1, \ldots, 13\}$, and $M^{\prime}$ has no 4element circuit that contains $\left\{w_{i}, w_{j}\right\}$ and no 5-element circuit that contains $\left\{w_{1}, w_{i}, w_{j}\right\}$ for all $i, j \in\{2, \ldots, 13\}$.

Part (i) follows immediately from Lemma 4.5.8. For parts (ii) and (iii) we first apply Lemma 4.5 .6 to find $w_{2}$, then we repeatedly apply Lemma 4.5.7. The bounds in (ii) allow for having to shift each $m$-block twice to avoid $x$ or $y$, and hence any triangles. Solving the recurrence relation for $k_{j}$ in (ii) gives $k_{13} \leq 663$. 
Now, by Lemma 4.5.8, we can choose the first element $w_{1}$. Consider the elements $w_{2}, \ldots, w_{13}$. At most three of $w_{2}, \ldots, w_{13}$ are in 4-element circuits that contain $w_{1}$ and meet $\{a, b\}$ by Lemma 4.5.9, at most one of $w_{2}, \ldots, w_{13}$ can be in $\operatorname{cl}_{M}\left(P_{1} \cup\right.$ $a)$, and at most five of $w_{2}, \ldots, w_{13}$ are in triangles that meet $\{a, b\}$ by Lemma 4.5.8. Thus there are pairs $w_{1}, w_{i}$ for at least three $i \in\{2, \ldots, 13\}$ with the desired properties.

\subsection{The bound}

We assume that we have chosen $v, w_{1}, w_{2}, w_{3}$ according to Lemma 4.5.10. Suppose that $M / v, w_{i}$ is not fragile. Then it follows from Theorem 4.2.10 that $M$ has a 4 or 5-element bad circuit $C$ of $M$. If $M$ has a 4-element bad circuit, then we proved in Theorem 4.2.11 that $M$ is in a bounded equivalence class of excluded minors (see 2.7.10.1). Thus we can assume that $C=\left\{v, w_{i}, x_{i}, y_{i}, z_{i}\right\}$, and that $\left\{v, x_{i}, y_{i}\right\}$ or $\left\{w_{i}, x_{i}, y_{i}\right\}$ is a triad of $M$.

We have to show that at least one of the minors $M / v, w_{i}$ does not have a bad circuit.

Lemma 4.6.1. If $M / v, w_{i}$ is 3-connected with an $N$-minor, and $C$ is a bad circuit of $M$, then $C$ contains at most one element of $\{a, b\}$.

Proof. Seeking a contradiction, suppose that $\{a, b\} \subseteq C$. Then at least one element of $\{a, b\}$ is contained in a triad of $M$, so $M \backslash a, b$ is not 3-connected; a contradiction because $M \backslash a, b$ is 3-connected.

Next we show that no bad circuit can avoid both $a$ and $b$.

Lemma 4.6.2. If $C$ is a bad circuit of $M$ for the pair $v, w_{i}$, then $C$ contains at least one element of $\{a, b\}$.

Proof. Seeking a contradiction, suppose that $\{a, b\} \cap C=\emptyset$. Then $C$ is a 5-element circuit of $M \backslash a, b$, and $C$ contains a triad $T$ of $M \backslash a, b$ such that $T$ meets $\left\{v, w_{i}\right\}$. Now we know that $T \cup\left\{v, w_{i}\right\}$ has 4 internal elements of the path $\left(P_{1}, \ldots, P_{n}\right)$ by Lemma 4.5.5, and the ends of $T \cup\left\{v, w_{i}\right\}$ are both coguts elements. But then $C$ must have an end that is a coguts element; a contradiction of Lemma 4.5.2.

We can now show that $M$ has a contraction pair with no bad circuit. 
Lemma 4.6.3. There is some $w_{i} \in\left\{w_{1}, w_{2}, w_{3}\right\}$ such that $M$ has no bad circuit $C_{i}=\left\{v, w_{i}, x_{i}, y_{i}, z_{i}\right\}$ for the pair $v, w_{i}$.

Proof. Suppose that $M$ has a bad circuit $C_{i}=\left\{v, w_{i}, x_{i}, y_{i}, z_{i}\right\}$ for each $i \in\{1,2,3\}$. It follows from Lemma 4.6.1 and Lemma 4.6.2 that $\{a, b\} \cap C_{i} \cap C_{j} \neq \emptyset$ for some $i, j \in\{1,2,3\}$. Now by circuit elimination on the common element of $\{a, b\}$ in $C_{i}$ and $C_{j}$ there is a circuit $C$ of $M$ such that $C^{\prime \prime} \subseteq C_{i} \cup C_{j}-\{a, b\}$. Thus $C \subseteq$ $\left\{v, w_{i}, w_{j}, x_{i}, x_{j}, y_{i}, y_{j}\right\}$ is a circuit of $M \backslash a, b$. Moreover, $T_{i}=\left\{p, x_{i}, y_{i}\right\}$ and $T_{j}=$ $\left\{q, x_{j}, y_{j}\right\}$ are triads of $M \backslash a, b$ for some $p \in\left\{v, w_{i}\right\}$ and $q \in\left\{v, w_{j}\right\}$. We may assume that labels are chosen so that $x_{i}, x_{j}$ are guts elements and $y_{i}, y_{j}$ are coguts elements. We now analyse the possible cases.

Suppose $\left\{p, x_{i}, y_{i}\right\}=\left\{q, x_{j}, y_{j}\right\}$. Then $M^{\prime}$ has a circuit $C \subseteq\left\{v, w_{i}, w_{j}, x_{i}, y_{i}\right\}$; a contradiction of the choice of $v, w_{i}, w_{j}$. Thus we can assume that the triads contained in $C_{i}$ and $C_{j}$ are distinct.

Suppose $C_{i}$ has triad $T_{i}=\left\{v, x_{i}, y_{i}\right\}$. Then since $v<w_{i}<w_{j}$ it follows that $w_{j} \notin$ $T_{i}$. Then $C_{j}$ meets $\left\{x_{i}, y_{j}\right\}$ by orthogonality with $T_{i}$, so $y_{i}=y_{j}$. Suppose that $T_{j}=\left\{w_{j}, x_{j}, y_{j}\right\}$. Then $C_{i}$ meets $T_{j}$ in a single element $y_{j}$; a contradiction of orthogonality. Thus $T_{j}=\left\{x, x_{j}, y_{j}\right\}$ where $x_{i} \neq x_{j}$ and $y_{i}=y_{j}$. Then the circuit $C$ of $M^{\prime}$ must end in $w_{i}$ or $w_{j}$; a contradiction of Lemma 4.5.2.

We can now assume that $C_{i}$ has triad $T_{i}=\left\{w_{i}, x_{i}, y_{i}\right\}$. Suppose that $T_{j}=\left\{v, x_{j}, y_{j}\right\}$. Then $C_{i}$ meets $T_{j}$ in $v$, so $\left\{x_{j}, y_{j}\right\}$ meets $T_{i}$ by orthogonality. If $\left\{x_{j}, y_{j}\right\}$ meets $T_{i}$ in a single element, then $C_{j}$ meets $T_{i}$ in a single element; a contradiction of orthogonality. Thus $\left\{x_{j}, y_{j}\right\} \subseteq T_{i}$. Then $T_{i} \cup T_{j}$ is a 4-cosegment, so the circuit $C$ of $M^{\prime}$ must end in $w_{2}$; a contradiction of Lemma 4.5.2. Thus we can assume that $T_{j}=\left\{w_{j}, x_{j}, y_{j}\right\}$. If $T_{i}$ and $T_{j}$ meet in a single element, then we contradict orthogonality with $C_{i}$ or $C_{j}$. If they meet in two elements then $C$ contradicts Lemma 4.5.2. Assume that $T_{i}$ and $T_{j}$ are disjoint. By Lemma 4.5.2 $C$ must end in $x_{i}$ and $x_{j}$. Then $C=\left\{x_{i}, s, t, x_{j}\right\}$ for some $s \in\left\{w_{i}, y_{i}\right\}$ and $t \in\left\{w_{j}, y_{j}\right\}$.

Now $x_{j} \in C$ and $x_{j} \in C_{j}$, so by elimination there is a circuit $C^{\prime}$ of $M$ such that $C^{\prime} \subseteq\left(C \cup C_{j}\right)-\left\{x_{j}\right\}$. Now since $C^{\prime}$ is not properly contained in $C_{j}$, it follows that $C^{\prime}$ meets $\left\{x_{i}, s\right\}$. By orthogonality $C^{\prime}$ contains $\left\{x_{i}, s\right\}$. Similarly, $C^{\prime}$ is not properly contained in $C_{i}$ so $C^{\prime}$ meets $\left\{w_{j}, y_{j}\right\}$. Then by orthogonality with the triad $T_{j}$, it follows that $C^{\prime}$ contains $\left\{w_{j}, y_{j}\right\}$. Now if $C^{\prime}$ avoids the common element $c \in\{a, b\}$ in both $C_{i}$ and $C_{j}$, then $C^{\prime}$ is a circuit of $M^{\prime}$ that contradicts Lemma 4.5.2. Thus $\left\{c, x_{i}, s, y_{j}, w_{j}\right\} \subseteq C^{\prime}$. Now consider the circuits $C^{\prime}$ and $C_{i}$ or $M$. By elimination 
on $c$, it follows that $M^{\prime}$ has a circuit $C^{\prime \prime}$ contained in $\left\{v, w_{i}, x_{i}, y_{i}, y_{j}, w_{j}\right\}$. But $C^{\prime \prime}$ is contained in the internal elements of the path and $C^{\prime \prime}$ has at most one guts element, so some end of $C^{\prime \prime}$ is a coguts element; a contradiction of Lemma 4.5.2.

The following Theorem gives the final bound on $M$.

Theorem 4.6.4. Let $M$ be a robust excluded minor for the class of matroids representable over $\mathbb{H}_{5}$ or $\mathbb{U}_{2}$. If $M$ has some pair of elements $a, b$ such that $M \backslash a, b$ is 3-connected with a $\left\{U_{2,5}, U_{3,5}\right\}$-minor, then $|E(M)| \leq 1334$ or there is some excluded minor $M^{\prime}$ that is $\Delta$ - $\nabla$-equivalent to $M$ or $M^{*}$ such that $\min \left\{r\left(M^{\prime}\right), r^{*}\left(M^{\prime}\right)\right\} \leq 10$.

Proof. Suppose that $M$ has a pair of elements $a, b$ such that $M \backslash a, b$ is 3-connected with a $\left\{U_{2,5}, U_{3,5}\right\}$-minor. Seeking a contradiction, we suppose that $|E(M)| \geq$ 1334 and that there is no excluded minor $M^{\prime}$ that is $\Delta$ - $\nabla$-equivalent to $M$ or $M^{*}$ that satisfies $\min \left\{r\left(M^{\prime}\right), r^{*}\left(M^{\prime}\right)\right\} \leq 10$. Then it follows from Theorem 4.2.11 and the robustness of $M$ that $M \backslash a, b$ is a $\left\{U_{2,5}, U_{3,5}\right\}$-fragile matroid. Furthermore, it follows from Lemma 4.3.6 that $M \backslash a, b$ has an $\left\{X_{8}, Y_{8}, Y_{8}^{*}\right\}$-minor. Now by Theorem 4.2 .5 there is a path sequence for $M \backslash a, b$ with corresponding path of 3-separations $\left(P_{1}, \ldots, P_{n}\right)$. We may assume that the path of 3-separations was chosen such that $P_{1}$ is a triad of $M \backslash a, b$ by Lemma 4.4.4, and we may also assume that the element $a$ blocks the 3-separation $\left(P_{1}, P_{2} \cup \cdots \cup P_{n}\right)$ of $M \backslash a, b$ by Lemma 4.4.5. Now $\left|P_{1} \cup P_{2}\right|=4$ and $\left|P_{n-1} \cup P_{n}\right| \leq 6$ by Lemma 4.4.4. Since $1332 \leq|E(M)-\{a, b\}|=\sum_{i=1}^{n}\left|P_{i}\right|$, we have $1322 \leq \sum_{i=3}^{n-2}\left|P_{i}\right|$. Moreover, we have $\left|P_{i}\right| \leq 2$ by Lemma 4.3.4 and Lemma 4.4.2, so $n \geq 665$. Now, by Lemma 4.5.10 and Lemma 4.6.3, we can find a pair of internal coguts elements $c, d$ of $M \backslash a, b$ such that $M \backslash a, b / c, d$ and $M / c, d$ are 3-connected with a $\left\{U_{2,5}, U_{3,5}\right\}$-minor, the element $a$ blocks the triad $P_{1}$ of $M \backslash a, b / c, d$, and $M$ has no 4- or 5-element circuit containing $c, d$. Then it follows from Theorem 4.2.10 and the robustness of $M$ that $M / c, d$ is a $\left\{U_{2,5}, U_{3,5}\right\}$-fragile matroid. But then $M \backslash a, b / c, d$ is a $\left\{U_{2,5}, U_{3,5}\right\}$ fragile matroid with an extension $M / c, d$ such that $M / c, d$ is $\left\{U_{2,5}, U_{3,5}\right\}$-fragile and the element $a$ blocks the triad $P_{1}$ of $M \backslash a, b / c, d$; a contradiction of Theorem 4.2 .5 .

Since $\mathbb{H}_{5}$ and $\mathbb{U}_{2}$ are finitary partial fields, we have the following Corollary. 
Corollary 4.6.5. There are finitely many robust excluded minors $M$ for the class of matroids representable over $\mathbb{H}_{5}$ or $\mathbb{U}_{2}$ with the property that $M$ has a pair of elements $a, b$ such that $M \backslash a, b$ is 3-connected with a $\left\{U_{2,5}, U_{3,5}\right\}$-minor.

Proof. Suppose that $M$ is a robust excluded minor that has a pair of elements $a, b$ such that $M \backslash a, b$ is 3 -connected with a $\left\{U_{2,5}, U_{3,5}\right\}$-minor. It follows from Theorem 4.6 .4 that either $|E(M)| \leq 1334$ or there is some excluded minor $M^{\prime}$ that is $\Delta$ - $\nabla$-equivalent to $M$ or $M^{*}$ that satisfies $\min \left\{r\left(M^{\prime}\right), r^{*}\left(M^{\prime}\right)\right\} \leq 10$. There are finitely many matroids $M$ such that $|E(M)| \leq 1334$. On the other hand, suppose that $M$ or $M^{*}$ is $\Delta$ - $\nabla$-equivalent to an excluded minor $M^{\prime}$ that satisfies $\min \left\{r\left(M^{\prime}\right), r^{*}\left(M^{\prime}\right)\right\} \leq 10$. Then $|E(M)|=\left|E\left(M^{\prime}\right)\right|$, and $M^{\prime}$ is a single-element extension or coextension of some 3 -connected $\mathbb{H}_{5}$ - or $\mathbb{U}_{2}$-representable minor with rank or corank at most 10 . Now every $\mathbb{H}_{5}$ - or $\mathbb{U}_{2}$-representable matroid is also $\mathrm{GF}(5)$-representable, so it follows that $|E(M)| \leq\left(5^{10}-1\right) /(5-1)+1$ by Lemma 4.2.15, and there are finitely many such matroids. 


\section{Bibliography}

[1] Brettell, N., And Semple, C. A splitter theorem relative to a fixed basis. Annals of Combinatorics 18, 1 (2014), 1-20.

[2] Chun, C., Chun, D., Clark, B., Mayhew, D., Whittle, G., And VAN ZWAM, S. Computer-verification of the structure of some classes of fragile matroids, arXiv:1312.5175.

[3] Chun, C., Chun, D., Mayhew, D., and van Zwam, S. H. Fanextensions in fragile matroids, arxiv:1312.5401.

[4] Chun, C., Mayhew, D., Whittle, G., and van Zwam, S. The structure of binary Fano-fragile matroids, in preparation.

[5] Geelen, J., Gerards, A., And Kapoor, A. The excluded minors for GF(4)-representable matroids. Journal of Combinatorial Theory, Series B 79,2 (2000), $247-299$.

[6] Geelen, J., Gerards, B., And Whittle, G. Solving Rota's Conjecture. Notices of the AMS 61, 7.

[7] Geelen, J., Gerards, B., And Whittle, G. Structure in minor-closed classes of matroids. Surveys in Combinatorics (2013), 327-362.

[8] Geelen, J., Oxley, J., Vertigan, D., And Whittle, G. Weak maps and stabilizers of classes of matroids. Advances in Applied Mathematics 21, 2 (1998), 305-341.

[9] Geelen, J., And Whittle, G. Matroid 4-connectivity: A deletioncontraction theorem. Journal of Combinatorial Theory, Series B 83, 1 (2001), 15-37. 
[10] Geelen, J., AND WhitTle, G. Certifying non-representability of matroids over prime fields. submitted for publication (2011).

[11] Gubser, B. S. A characterization of almost-planar graphs. Combinatorics, Probability and Computing 5, 03 (1996), 227-245.

[12] Hall, R., Mayhew, D., and Van Zwam, S. H. The excluded minors for near-regular matroids. European Journal of Combinatorics 32, 6 (2011), 802-830.

[13] Hall, R., Oxley, J., Semple, C., And Whittle, G. Forkdecompositions of matroids. Advances in Applied Mathematics 32, 3 (2004), $523-575$.

[14] KAHN, J. On the uniqueness of matroid representations over GF(4). Bull. London Math. Soc 20, 5 (1988), 10.

[15] Kingan, S., And Lemos, M. Almost-graphic matroids. Advances in Applied Mathematics 28, 3 (2002), 438-477.

[16] Mayhew, D., Newman, M., and Whittle, G. On excluded minors for real-representability. Journal of Combinatorial Theory, Series B 99, 4 (2009), 685-689.

[17] Mayhew, D., And Royle, G. F. Matroids with nine elements. Journal of Combinatorial Theory, Series B 98, 2 (2008), 415-431.

[18] MaYhew, D., AND VAN ZWAM, S. On excluded minors for dyadic matroids, in preparation.

[19] Mayhew, D., van Zwam, S., And Whittle, G. Stability, fragility, and Rota's Conjecture. Journal of Combinatorial Theory, Series B 102, 3 (2012), 760-783.

[20] OxLeY, J. Matroid theory, Second Edition. Oxford University Press, New York, 2011.

[21] Oxley, J., Semple, C., And Vertigan, D. Generalized $\Delta-Y$ exchange and $k$-regular matroids. Journal of Combinatorial Theory, Series B 79, 1 (2000), 1-65. 
[22] Oxley, J., Semple, C., And Whittle, G. Maintaining 3-connectivity relative to a fixed basis. Advances in Applied Mathematics 41, 1 (2008), $1-9$.

[23] Oxley, J., Vertigan, D., And Whittle, G. On inequivalent representations of matroids over finite fields. journal of combinatorial theory, Series B 67, 2 (1996), 325-343.

[24] OXLEy, J., AND WU, H. On the structure of 3-connected matroids and graphs. European Journal of Combinatorics 21, 5 (2000), 667-688.

[25] OXLEY, J. G. A characterization of a class of non-binary matroids. Journal of Combinatorial Theory, Series B 49, 2 (1990), 181-189.

[26] Pendavingh, R., And VAn Zwam, S. The Hydra- $k$ partial fields, arXiv:1003.1640v1.

[27] Pendavingh, R., And van Zwam, S. Confinement of matroid representations to subsets of partial fields. Journal of Combinatorial Theory, Series B 100, 6 (2010), 510-545.

[28] Pendavingh, R., AND VAn Zwam, S. Lifts of matroid representations over partial fields. Journal of Combinatorial Theory, Series B 100, 1 (2010), $36-67$.

[29] Pendavingh, R., Van Zwam, S., ET Al. Sage Matroid Package, included in Sage Mathematics Software 6.2. The Sage Matroid Development Team, 2014. http://www.sagemath.org.

[30] Robertson, N., And Seymour, P. D. Graph minors. xvi. excluding a non-planar graph. Journal of Combinatorial Theory, Series B 89, 1 (2003), 43-76.

[31] Semple, C., And Whittle, G. Partial fields and matroid representation. Advances in Applied Mathematics 17, 2 (1996), 184-208.

[32] Semple, C. A. k-regular matroids. PhD thesis, Victoria University of Wellington, 1998.

[33] Stein, W., ET AL. Sage Mathematics Software (Version 6.2). The Sage Development Team, 2014. http://www.sagemath.org. 
[34] Truemper, K. A decomposition theory for matroids. vi. almost regular matroids. Journal of Combinatorial Theory, Series B 55, 2 (1992), 253-301.

[35] van Zwam, S. H. Partial fields in matroid theory. $\mathrm{PhD}$ thesis, $\mathrm{PhD}$ thesis, Technische Universiteit Eindhoven, 2009.

[36] Whittle, G. On matroids representable over GF(3) and other fields. Transactions of the American Mathematical Society 349, 2 (1997), 579-603.

[37] Whittle, G. Stabilizers of classes of representable matroids. Journal of Combinatorial Theory, Series B 77, 1 (1999), 39 - 72.

[38] Whittle, G., AND Williams, A. On preserving matroid 3-connectivity relative to a fixed basis. European Journal of Combinatorics 34, 6 (2013), 957-967.

[39] Williams, A. Detachable pairs in 3-connected matroids. PhD thesis, Victoria University of Wellington, Submitted 2014. 


\section{Index}

algebraically-equivalent, 11

allowable

cosegment, 66

parallel extension, 67

pivot, 27, 106

segment, 66

series extension, 67

set, 66

bad

cocircuit, 57

submatrix, 27

basis, 16

blocks, 81, 113

coguts, 46

companion matrix, 25

cosegment, 66

$\Delta-\nabla$

-equivalent, 17

-step, 67

equivalent

$\mathbb{P}$-representations, 11

finitary, 10

full closure, 66

gadget, 33

generalized

$\Delta$ - $\nabla$-exchange, 12 parallel connection, 12

geometrically-equivalent, 11

gluing a wheel, 67

good separation, 45

guts, 46

$\mathbb{H}_{5}, 13$

-representations, 13

incriminating set, 26

$(N, B)$

-robust, 16

-strong, 16

N

-stable, 24

$\mathscr{N}$

-allowable, 66

-contractible, 15, 62

-deletable, 15, 62

-essential, 15, 62

-flexible, 15, 62

-fragile, 15, 62

-minor, 62

$P$-fan relative to $B, 40$

$\mathbb{P}, 8$

-matrix, 9

-representable, 9

-representation, 9

-stabilizer, 11, 24

partial field, 8 
automorphism, 10

homomorphism, 9

isomorphism, 10

path

-generating, 66, 76

conforming, 115

of 3-separations, 66

sequence, 67, 77

end, 77

rearrangement, 78

step, 77

pivoting, 10

robust basis, 17, 109

$S^{\prime}, 40$

scaling-equivalent, 11

segment, 66

strong $\mathbb{P}$-stabilizer, 12, 25

submatrix, 9

type-I fan relative to $B, 40$

type-II fan relative to $B, 40$

$\mathbb{U}_{2}, 14$

uncrossing, 20

uniquely representable, 11

unstable, 29

z-closed, 40 